\title{
Transcriptome analysis of programmed cell death associated with allorecognition in chestnut blight fungus \\ Cryphonectria parasitica
}

by

\begin{abstract}
Anatoly Belov
A thesis submitted to the Faculty of Graduate and Postdoctoral Affairs in partial fulfillment of the requirements for the degree of

Doctor of Philosophy
\end{abstract}

in

Biology

Carleton University

Ottawa, Ontario

(C) 2017

Anatoly Belov 
The "dialectics" of this situation is that the evolutionary narratives certainly are oversimplified "myths" that have the unfortunate (and, in modern studies, unintended) teleological flavor (as in "selected for" or, worse, "selected for the purpose of"), yet the language of these narratives seems best suited to describing evolution and formulating falsifiable hypotheses that propel further research. At present, we hardly can give up these stories [...] precisely because they are necessary means for the advancement of research, even though they tend to leave a scientist [...] with feelings of uneasiness and dissatisfaction. It seems important not to forget that evolutionary narratives effectively are semantic devices that are constructed to structure and simplify our thinking about evolution and to facilitate the generation of hypotheses. These narratives should be prudently distrusted and by no account should be construed as "accurate representations of reality" (whatever that might mean [...]).

Eugene V. Koonin

"The Logic Of Chance, The Nature and Origin of Biological Evolution", p. 439 


\section{Abstract}

In this thesis, I analysed transcriptional profiles of the chestnut blight fungus, Cryphonectria parasitica, during vegetative incompatibility (allorecognition) reactions due to differences at the vic3 locus. Out of a total 13944 identified expressed transcripts, including 2334 novel ones, only 1411 were differentially expressed during vic3 incompatibility reaction. Functional enrichment analysis showed increased expression of genes involved in detoxification and stress response (e.g.s Cytochrome p450, Glutathione S-transferase), and toxin biosynthesis. Surprisingly, even though the test strains were both the same mating type (MAT-2), genes involved in sexual reproduction $(m f 2-1, m f 2-2$ and mat-2) showed the most dramatic increase in expression during allorecognition response. Further qPCR analysis showed that activation of mating pheromone genes occurs during incompatible reactions involving five of the six known vic incompatibility loci. The only exception was vic4, which elicits a weak incompatibility and showed almost no change in pheromone gene expression. Analysis suggests that $m f 2-1, m f 2-2$ and mat-2 expression is triggered by activation of asexual sporulation. Genes encoded at the vic3 locus, vic $3 a$ and vic $3 b$, were upregulated in barraging samples along with seven HET-domain genes located at other regions of the genome. Among the seven HET genes activated, one is located at the vicl locus and previously implicated in vicl incompatibility. Activation of these same HET domain genes also occurred in other vic incompatible pairings. For example, upregulation of $\operatorname{dev} 3 a$ and vicla genes occurred during incompatibility reactions associated with each of the six known vic loci. This suggests that some HET genes serve as universal allorecognition factors. Furthermore, this data indicates that each incompatibility locus uses a set of several HET genes to 
activate Programmed Cell Death (PCD). In addition, I analysed the effects on barrage formation of $\mathrm{p} 29$, a protein-coding region from Cryphonectria hypovirus 1 (CHV1). Expression of p29 in C. parasitica was previously shown to delay the onset of vic3associated PCD. Results of the analysis indicated that ectopically expressed p29 does not have a strong modifying effect on gene expression in barraging strains. This study illustrates that nonself recognition is an active defence mechanism, where stress response and detoxification are combined with mycotoxins production. 


\section{Acknowledgements}

First of all, I want to thank Myron Smith. Thank you for giving me an opportunity to work with you. Thank you for your constant support and countless hours you spent helping me to get out of dead ends. Without you so many good things in my life over the last 6 years couldn't have happened. You taught me how to do research and showed me what it means to be a good person.

For six years working in Myron's team I was surrounded by wonderful colleagues. Denis Lafontaine, Ryan Reshke, Imelda Galvan, Isabel Cruz, Chanchal Yadav, Abbey Laoye thank you for your support, skill sharing and friendship.

I would like specially thank Nicolas Rodrigue and Bill Willmore. Thank you for your valuable advice and guidance in bioinformatics and proteomics fields. I want to express my sincere gratitude to the members of committee, Linda Bonen, John Vierula James Green and Sven Saupe, thank you for your time and advice. I would also like to thank Ashkan Golshani, Alex Wong and other scientists in the department who helped with advice, equipment and materials when they were the most needed.

A special note to my family, to my Mom and Dad, who always are supportive and understanding in all my endeavours. Finally, this work would not be possible without the single most important person in my life, Grigory Chudov. All software related parts of this work would not be as good without your help. Thank you for supporting me in my decision to follow a science path and always being there for me. Thank you for your love and friendship. 
This work was partially funded by Carleton University, Ontario Trillium Scholarship, Natural Sciences and Engineering Research Council (NSERC). 


\section{Table of Contents}

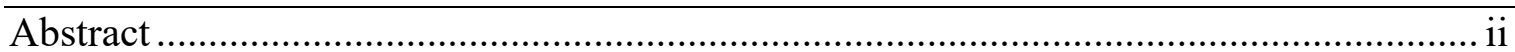

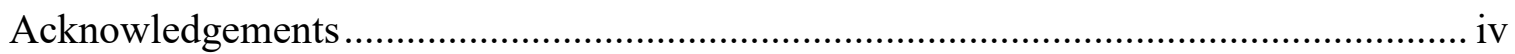

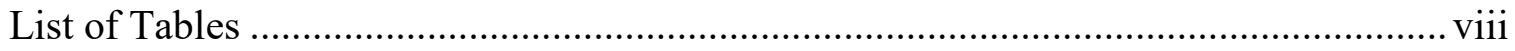

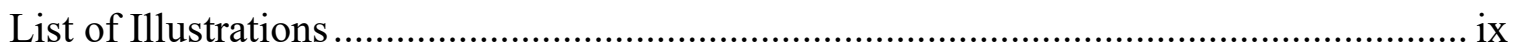

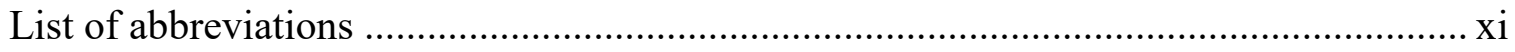

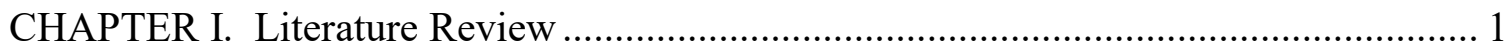

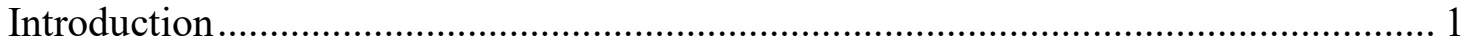

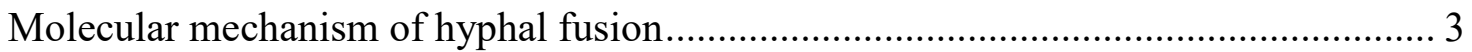

Vegetative incompatibility ............................................................................... 7

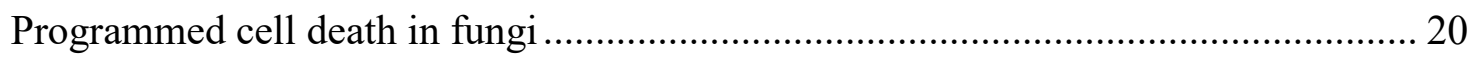

Cryphonectria parasitica as a model organism ……….............................................. 23

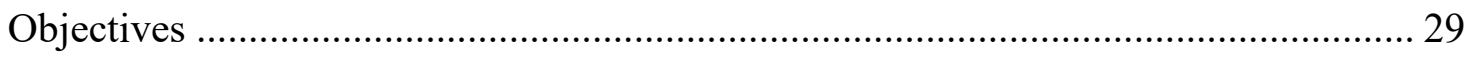

CHAPTER II. RNA-seq analysis of vic3-associated barrage formation ............................ 31

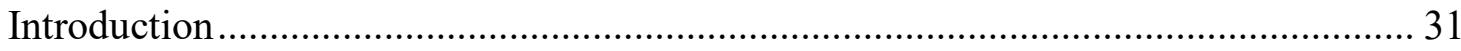

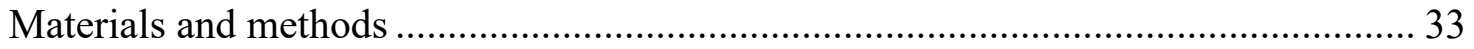

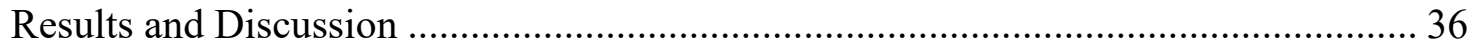

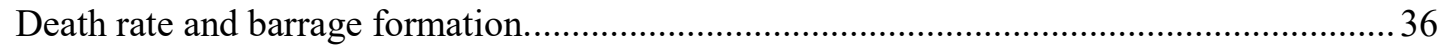

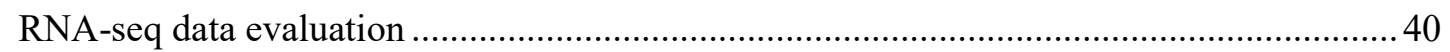

Principal component analysis and Functional annotation .................................................... 42

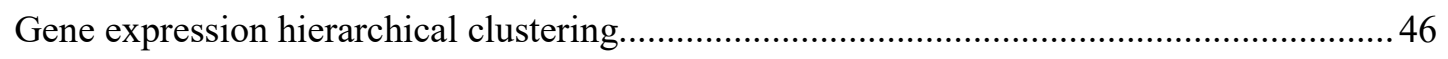

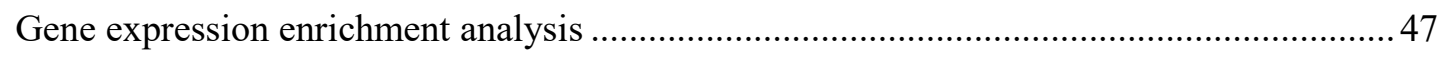

Comparison to HI transcription profiles of $N$. crass $a$ and $P$. anserina .................................65

Conclusion ................................................................................................... 79

CHAPTER III. Effects of CHV1-EP713 p29 protein and Dicer-2 knockout on barrage

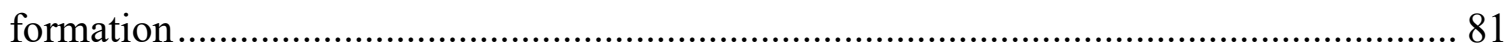

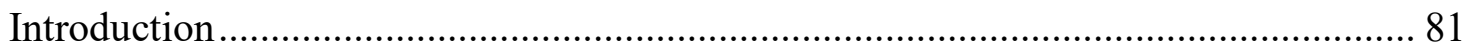




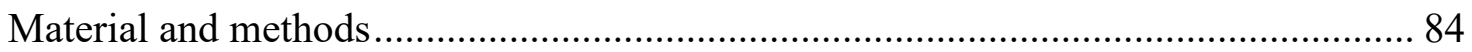

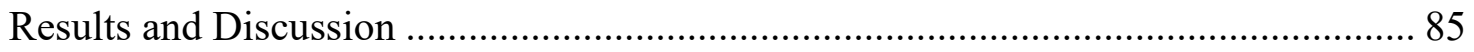

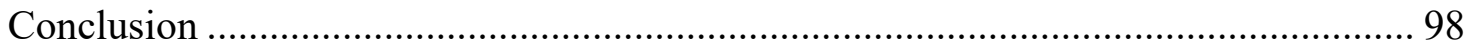

CHAPTER IV. Expression profile of HET genes during allorecognition in

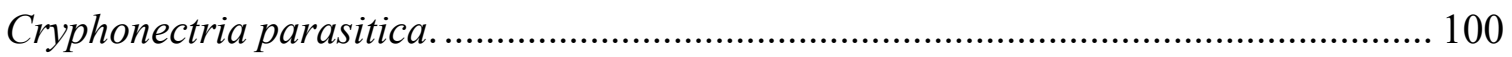

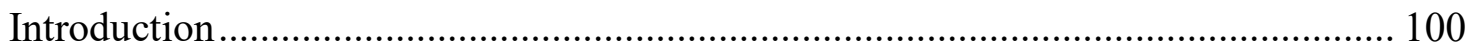

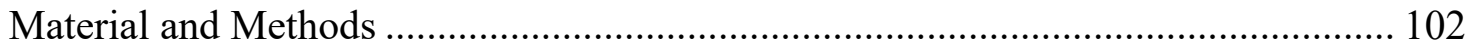

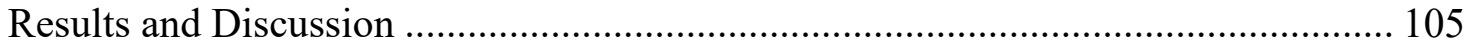

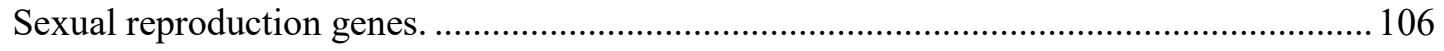

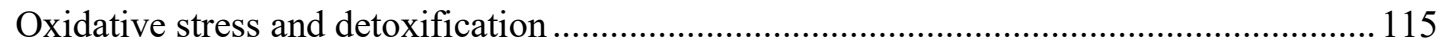

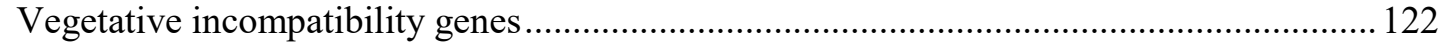

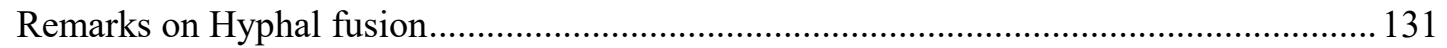

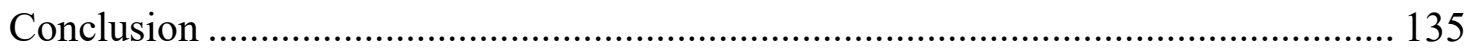

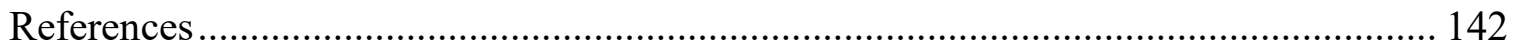

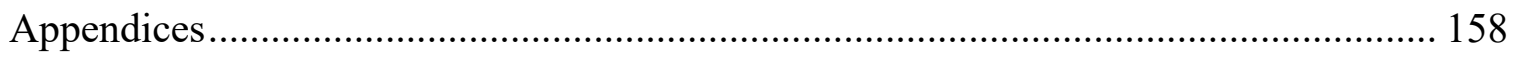




\section{List of Tables}

Table II - 1. Spectrophotometry analysis of total RNA extracted from barraging and control samples.

Table IV - 1. Primers used for quantitative reverse polymerase quantitative chain reaction (RT-qPCR). 104

Table S 1. Hierarchical clustering analysis of differentially expressed genes. Table shows only those clustered DE genes that were mentioned in text. 158 Table S 2. DAVID enrichment analysis groups of GO terms and INTERPRO/Pfam domains. 161

Table S 3. Orthologs differentially expressed during HI in P. anserina and N. crassa, and barrage in C. parasitica. 172 


\section{List of Illustrations}

Figure I - 1. Barrages formed at the confluence of vic3 incompatible strains of C.

parasitica.

Figure I - 2. Detection of cell death during incompatible interactions using Evans Blue assay.

Figure I - 3. Examples of genetic interaction types involved in vegetative incompatibility.

Figure I - 4. Generalized representation of proteins involved in innate nonself recognition in animals, plants and fungi. All these proteins activate PCD, when trigged by incompatibility. 17

Figure I - 5. Canker on chestnut bark showing exposed C. parasitica conidia 24

Figure I - 6. CHV1 genome organisation. Black arrows indicate autocatalysis point...... 27

Figure II - 1. Barrage formation and programmed cell death during vic3 incompatibility.

Figure II - 2. Annotation data of C. parasitica genome, where genes previously identified and novel transcripts were annotated with BLAST $+\left(\mathrm{e}-\mathrm{value}<10^{-10}\right)$

Figure II - 3. Principal components analysis (PCA) of normalised expression variance for intra-strain (blue dots) and inter-strain combinations (brown dots). 43

Figure II - 4. Hierarchical clustering of 1411 differentially expressed genes $(\log 2 \mathrm{FC}>2$,

p-value $<0.0001)$

Figure II - 5. (next page) Gene enrichment analysis of GO terms for biological process (A) and for INTERPRO/Pfam domains (B) 48

Figure II - 6. Orthologs of N. crassa and P. anserina identified in C. parasitica genome. 67 Figure II - 7. (next page) Gene differential expression (DE) shown on C. parasitica genome map (11 largest scaffolds).

Figure III - 1. Cell death rate estimations using fluorescent microscopy.... 86 
Figure III - 2. Normalised expression rates of Tetrahydroxynaphthalene reductase, HET domain genes (dev3 abij), laccase (including lac-1 and five probable laccases) and endothiapepsin genes during vic3 incompatibility

Figure IV - 1. Comparison of differential expression (DE) data acquired by RT-qPCR and RNA-seq of pheromone precursor genes in strains undergoing barrage formation compared to control strains in monoculture

Figure IV - 2. ROS production during vic3 incompatibility.

Figure IV - 3. Normalised expression rates of cpvib1, HET domain genes and vic genes during vic3 incompatibility. 126 Figure IV - 4. RNA-seq and RT-qPCR data of differential expression of four HET domain genes and cpvib1 gene under six types of incompatibility in C. parasitica Figure IV - 5. Expression of gene orthologs associated with hyphal fusion and G-protein signaling in C. parasitica.

Figure IV - 6. Molecular pathways during barrage formation in C. parasitica vic3 incompatible strains. 136 
ADH Alcohol Dehydrogenase

AIF Apoptosis Inducing Factor

APE AP enduclease 1

BCL2 B-cell lymphoma 2 protein family

CARD caspase requirement domain

CAT Conidial Anastomosis Tube

CHV1 Cryphonectria Hypovirus 1

DCF Dichlorofluorescein

DE Differential Expression

dev3 Differentially expressed during vic3 incompatibility

dNTP deoxynucleotide triphosphate

dsRNA Double-stranded RNA

EST Expressed Sequence Tag

FPKM Fragments Per Kilobase of transcript per Million mapped reads

GO Gene Ontology

GSH Glutathione

GST Glutathione S-transferase

HI Heterokaryon Incompatibility

HMG High Mobility Group box

IAP Inhibitor of Apoptosis Proteins

JNK c-Jun N-terminal kinase

LLR Leucine-reach repeat

MAPK Mitogen Activated Protein Kinases

MAT Mating type

MFS Major Facilitator Superfamily

NACHT NTP-binding and oligomerization domain (NAIP (neuronal apoptosis inhibitory protein), CIITA (MHC class II transcription activator), $\underline{H E T-E}$ (incompatibility locus protein from Podospora anserina) and TP1 (telomerase-associated protein))

NGS Next Generation Sequencing

NLR NOD-like receptor

NOD nucleotide-binding oligomerization domain 
ORF Open Reading Frame

PCA Principal Component Analysis

PCD Programmed Cell Death

PDA Potato Dextrose Agar

PDB Potato Dextrose Broth

PIWI P-element Induced Wimpy testis

PK Protein Kinase

QO Quinone Oxidoreductase

qPCR Quantitative Polymerase Chain Reaction

RNAi RNA interference

RNA-seq Whole transcriptome shotgun sequencing with NGS

RNR Ribonucleotide reductase

ROS Reactive Oxygen Species

RT-qPCR Reverse Transcription qPCR

SCD Short-Chain Dehydrogenases

STAND Signal Transduction ATPases with Numerous Domains

TIR Toll/interleukin-1 (IL-1) receptor domain

UV Ultra violet

VI Vegetative Incompatibility

vic Vegetative incompatibility locus 


\section{CHAPTER I. \\ Literature Review}

\section{Introduction}

Hyphal fusion, or anastomosis, in filamentous fungi is a process that occurs regularly during vegetative (mitotic) growth. In a fungal colony (a mycelium), there are no individual cells, as such, compared to plants and animals. The fungal mycelium is loosely compartmentalized by semipermeable separators called septa. This type of organization allows for the relatively free flow of organelles, including nuclei and mitochondria, and other cellular components throughout the entire mycelium. Forming interconnections between hyphae within the same mycelium may allow for an increase in individual organism fitness. For example, it may allow for more effective exchange of nutrition and organelles within the colony, which helps to allocate more energy resources to actively growing areas. Fusion may also happen between different fungal strains. If they are genetically similar, the two strains can fuse to form a single heterokaryotic mycelium. A heterokaryon is a cell or mycelium that contains genetically different nuclei. While exchange of nutrition is likely helpful, exchange of genetic material may not always be advantageous. Fusions between different strains can lead to infection by viruses and other infectious elements. Thus, it seems, fungi have evolved allorecognition mechanisms to identify nonself hyphal fusions and impede the spread of infectious agents. Allorecognition mechanisms that occur during vegetative growth are controlled by vegetative incompatibility (VI) systems. VI often manifests as a 'barrage' (separator, wall) that forms at the confluence of two incompatible strains (Figure I-1). The VI system is genetically determined and controlled by vic (vegetative incompatibility) loci. 


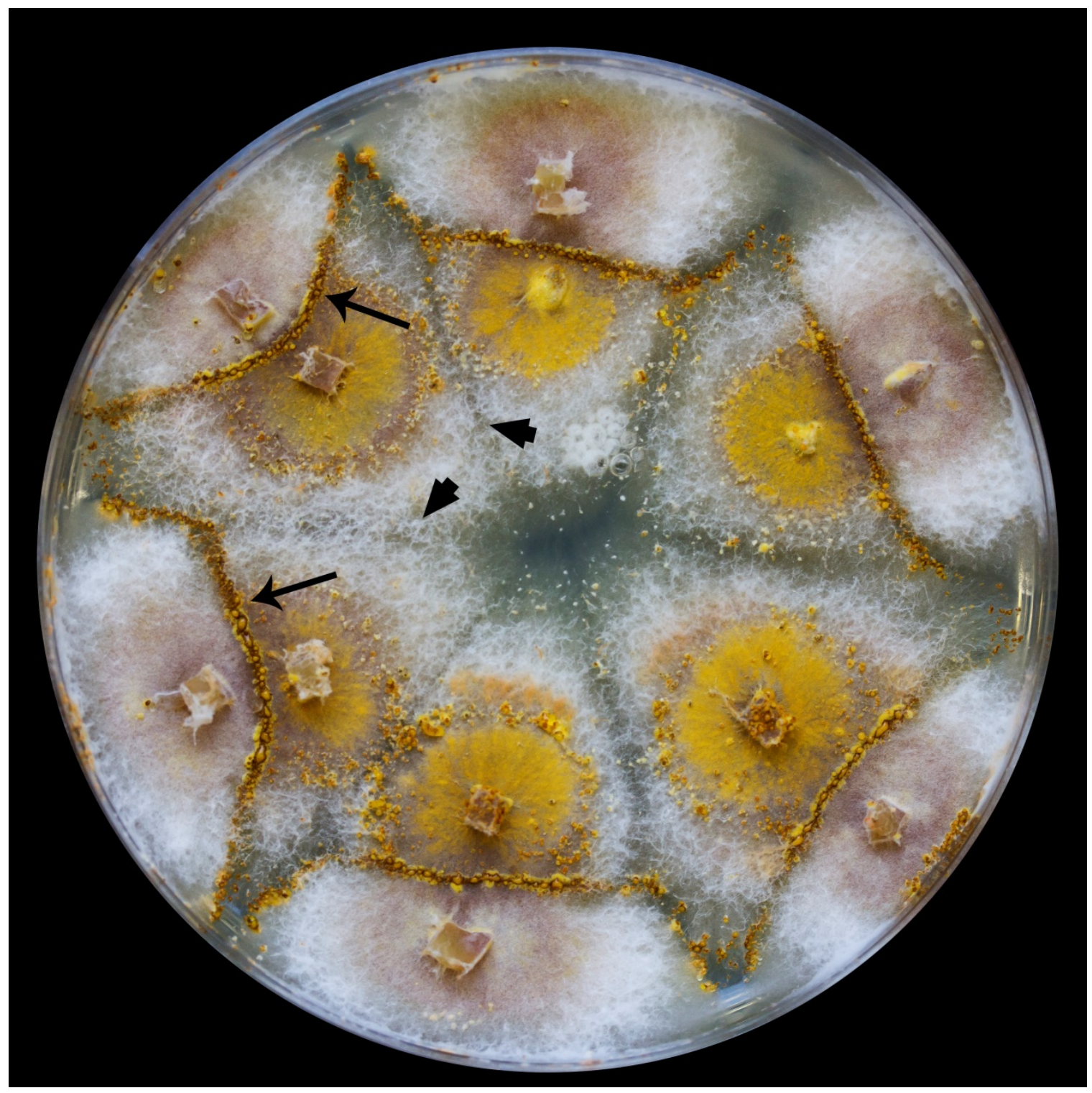

Figure I - 1. Barrages formed at the confluence of vic3 incompatible strains of $C$. parasitica.

EP155 (vic3-2) colonies around the edge of the plate interact with colonies of the six different vic3 incompatible strains (vic3-1). Barrage line between incompatible strains indicated by arrow. Compatible interactions shown by arrowheads. 
In general Ascomycota carry about a dozen of vic loci, when fused strains are carry different alleles in one or more vic loci it triggers activation of the VI system. During barrage, genetically incompatible strains fuse and Programmed Cell Death (PCD) is activated to result in formation of a wall of dead cells separating the two strains.

In this study, Cryphonectria parasitica (Murr.) Barr [=Endothia parasitica (Murr.) P.J. and H.W. Anderson] - the causal agent of chestnut blight - was used as a model to investigate molecular mechanisms behind vic3-associated nonself recognition.

\section{Molecular mechanism of hyphal fusion}

The mycelium of Ascomycota fungi is comprised of a hyphal network. Individual cells of a hypha are separated by an incomplete wall called a septum, through which nuclei and other organelles and cellular components are able to travel. The hyphal tips are the most metabolically active part of the mycelium and areas of most active growth can usually be found at the front edges of the colony, where young hyphae actively grow and branch to colonize new areas of substratum. Behind the active growth area, toward the center of the mycelium, hyphae undergo a process called anastomosis or hyphal fusion to enable interconnections throughout the mycelium. These anastomoses often involve tipto-tip contact between cells (Figure I-2). Hyphal fusion can also occur shortly after germination of conidia through a specialized structure called a 'conidial anastomosis tube' (CAT) that has been best characterized in N. crassa (Read et al. 2009). Figure I-2 (b, c) shows similar anastomosis tubes formed by $C$ parasitica hyphae. On a molecular level, formation of anastomosis involves chemotropic signaling and oscillatory protein complexes reacting to the signals (Fleißner et al. 2008). CAT allows individual conidial 

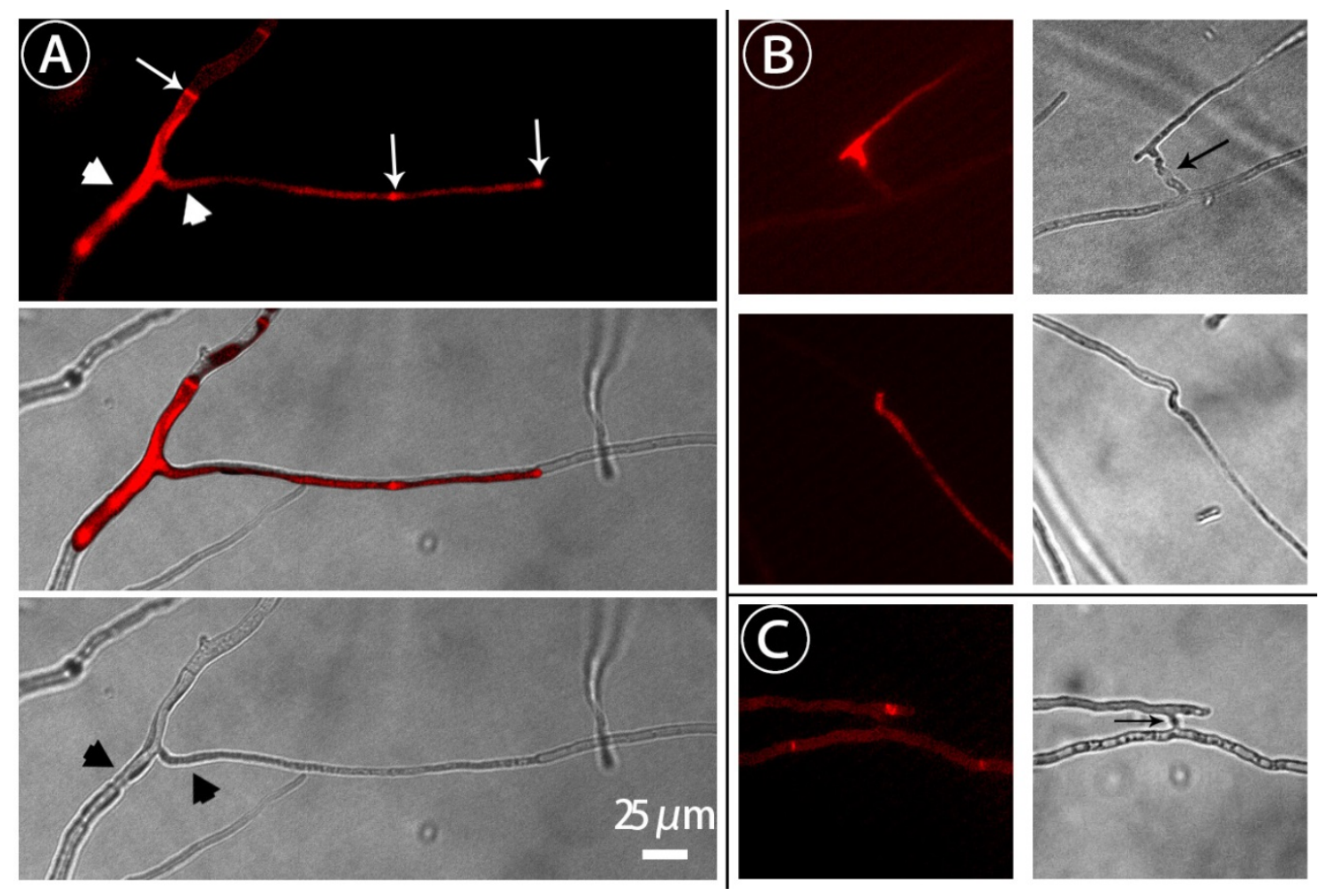

Figure I - 2. Detection of cell death during incompatible interactions using Evans Blue assay.

Dye accumulates only in dead cells and makes them glow under fluorescent light. A) Incompatible interaction under UV (top) shows glowing hyphae (arrowheads) from two incompatible strains fusing and undergoing cell death. Incompatible hyphae are compartmentalized by septa (narrow white arrows). Morphological changes in cell structure can also be seen under bright field illumination (bottom). The middle panel is an overlay of top (UV) and bottom (bright field) panels. B) Intermediate stage of incompatible interaction showing that individual strains differ in speed and onset of PCD. Left panel is under UV light, right panel is under bright field. At the time these Panel B images were acquired, only one of the two interacting hyphae is undergoing PCD. C) Early stage of incompatible interactions. Anastomosis tube (black arrows) formed between two hyphae and morphological changes of interacting hyphae (bright field, right) indicate incompatible interaction. However, under UV (left) hyphae do not show accumulation of Evan's Blue dye. 
germlings to form a continuous colony and act as an individual (Fleißner et al. 2009a). Hyphae are brought together through chemical signals, most probably associated with Gprotein coupled receptors, which facilitate the signal; the nature of these receptors, however, remains largely unknown (Jonkers et al. 2016). The overall model of self-fusion is referred to as "ping-pong signaling" or "cell dialog" (Figure I-3) (Fleißner et al. 2009a; Read et al. 2009). In this model, downstream regulation of signaling is associated with MAK-2 protein kinases pathway. Here, MAK-2 forms a complex with other protein kinases (PKs) aggregating at the tip of germling 'A'. At the same time, in the interacting germling 'B', a complex of proteins associated with SO (protein of unknown function), form and send a signal to germling ' $\mathrm{A}$ ' with the MAK-2 complex aggregated at the tip. In about 5-10 minutes, the cycle repeats, but this time germling ' $\mathrm{A}$ ' forms the SO complex and germling ' $\mathrm{B}$ ' forms the MAK-2 complex. Alteration of the two complexes and signaling continues until the two germlings fuse. As mentioned above, the chemical nature of the signaling molecule is unknown, but current modeling suggests that it requires only one signal molecule and receptor (Goryachev et al. 2012).

The downstream signaling for anastomosis following receptor activation is well studied in $N$. crassa and is usually referred as the MAK-2 pathway (Fleißner et al. 2009a). From the receptors to the MAPK, the cascade signal is transduced through PK STE-20, GTPase RAS-2, and capping protein CAP-1 (Dettmann et al. 2014). These activate a MAPK cascade similar to the one in yeast induced by sexual pheromones. In yeast, activation of G-protein receptor by pheromones leads to activation of PK Ste20p and scaffold protein Ste5p (Bhattacharyya et al. 2006). Activated by Ste20p, Ste11p forms a complex with Ste7p and Fus3p, which is held by scaffold protein Ste5p (Dan et 
al. 2001). During hyphal fusion in $N$. crassa similar molecular mechanisms are activated. Here, NRC-1 (ortholog of yeast Ste11p) binds with its adaptor protein STE-50 and forms a complex with MAK-2 (ortholog of yeast Ste7p) and MEK-2 (ortholog of yeast Fus3p). This complex is bound together by scaffolding protein HAM-5 (analog of yeast Ste5p). A combination of NRC-1/STE-50/MEK-2/MAK-2 and scaffolder HAM-5 forms a puncta, oscillating protein complex, that comes together due to the chemical signal from hypha with the activated SO, and dissociates through a feedback loop. SO protein is not well characterized and, so far, SO is not associated with any other known functions apart from those which are related to hyphal fusion (Fleißner et al. 2005; Fleißner and Herzog 2016).

MAK-2 targets are analogous to pheromones induced in yeast MAP kinase pathway (Maeder et al. 2007). In N. crassa, MAK-2 targets a PP-1 homolog of yeast, Ste12p, which is the ortholog of cpST12 in C. parasitica (Deng et al. 2007). In yeast, Ste12p is a transcription factor activated in response to mating pheromones, which leads to activation of mating or filamentation (Cook et al. 1996; Gustin et al. 1998). The role of Ste12p in sexual reproduction has been confirmed for various orthologs found in filamentous fungi. For example, in $N$. crassa, $p p-1$ is responsible for protoperithecia formation and vegetative growth ( $\mathrm{Li}$ et al. 2005). In contrast, in C. parasitica, $\mathrm{cpST} 12$ is required for female fertility; its deletion does not influence vegetative growth and development, but does significantly reduce virulence (Deng et al. 2007).

Finally, after hyphae are fused, the MAK-2 complex accumulates around the fusion pore, before the two cells completely merge. Fusion leads to exchange of cytoplasmic materials between hyphae and formation of a heterokaryon if the nuclei of the two fusing hyphae are genetically different. Within a colony this fusion process 
increases the interconnectedness, and in turn creates more effective flow of organelles and nutrition within a mycelium.

\section{Vegetative incompatibility}

Successful hyphal fusion results in the formation of a single, integrated individual, with a shared cellular content and genetic information. When genetically different strains fuse to form a heterokaryon, the resulting colony continues its life cycle as a single individual with mixed genetic and possibly new phenotypic features. Genetic differences at vic or het loci, however, restrict such mergers, and limit the exchange of genetic or cytoplasmic materials between individuals. This array of events, which leads to strain separation, is usually referred to as vegetative nonself recognition.

The most notable morphological outcome of vegetative nonself recognition is when two strains build up a separation wall - barrage - composed of dead cells in the region where two incompatible strains make contact (Figure I-1). The process that results in barrage can be called mycelial, somatic or vegetative incompatibility. Barrage often, but not always, is attributed to heterokaryon incompatibility (HI) (C. O. Micali and Smith 2003), the specific process whereby two incompatible nuclei cohabiting in the same cell results in slow growth or cell death. For example, two strains that differ at the vic4 locus in C. parasitica will form a barrage when confronted but can nevertheless fuse to yield a stable heterokaryotic mycelium (Smith et al. 2006). Normally, during HI, strains show reduced growth rate, abnormal morphology and eventually die due to PCD (Smith and Lafontaine 2013). 
VI (vegetative incompatibility, triggered by differences at vic loci/genes) and $\mathrm{HI}$ (heterokaryon incompatibility, triggered by differences at het loci/genes) are thus genetically related processes that occur during vegetative nonself recognition. In accordance with tradition, I will use the terms vegetative incompatibility (VI) and vic loci/genes when referring to $C$. parasitica vegetative incompatibility systems. The genetic determinants of $\mathrm{VI} / \mathrm{HI}$ appear to be quite variable between loci within fungal species and between species. Most incompatibility loci, however, share a common feature; they contain, or involve, a gene that encodes a HET domain (IPR010730) protein. Any given species can have more than 50 HET domain genes in the genome, but usually only those HET-domain genes that show genetic polymorphism within species can define allorecognition incompatibility (Glass and Dementhon 2006; Smith et al. 2000).

One of the most well studied organisms in relation to HI is $N$. crassa as demonstrated by the het-c/pin-c locus (Kaneko et al. 2006). This HI locus consists of two closely linked genes - het-c and pin-c - and three allelic forms of each gene have been identified (J. Wu and Glass 2001). The three alleles of pin-c show very high levels of genetic polymorphism, ranging from $40-50 \%$ similarity in amino acid content, except in the HET domain region where sequence similarity is $80-98 \%$. The molecular basis of incompatibility is not clear, but the mode of action is relatively simple. Incompatibility occurs through 'nonalleic' interactions when one allele of het-c is combined with an opposing allele of pin-c. For example, an HI reaction is triggered through interaction of het-cl with pin-c2, but not if het-cl is combined with pin-cl. Meanwhile, if the pin-c 

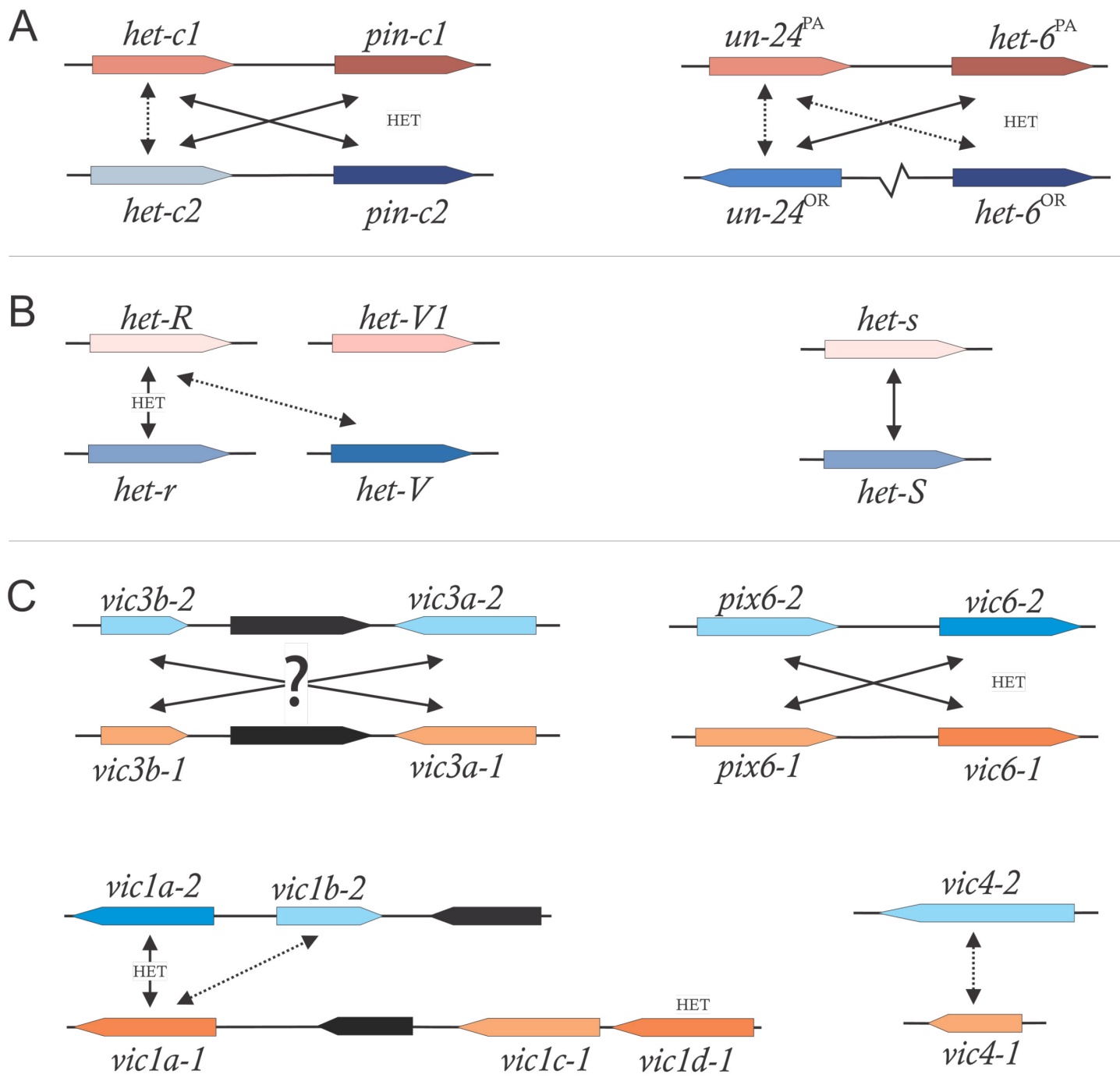

Figure I - 3. Examples of genetic interaction types involved in vegetative incompatibility.

Solid black arrows indicate strong VI interactions and dashed black arrows indicate weak VI interactions. Genes with HET domains are indicated. A) het-c and het- 6 are examples of VI loci from $N$. crassa. Within the het-c locus VI is triggered through 'nonallelic' interactions between het-c gene with pin-c (HET domain) gene from the opposite haplotype. Similarly, within the het-6 locus, VI is triggered by allelic interactions between $u n-24^{\mathrm{PA}}$ and $u n-24^{\mathrm{OR}}$ and by nonallelic interactions between $u n-24$ and het- 6 from the opposite haplotypes. B) Weak interactions between het- $R$ and het- $V$ from $P$. anserina is a type of temperature sensitive incompatibility. Here incompatible strains are able to form a heterokaryon when grown at $34^{\circ} \mathrm{C}$, but at $26^{\circ} \mathrm{C}$ VI is activated. The protein from allele het-s can produce a prion form that can interact with, and convert, the nonprion het-S protein into a pore-forming conformation. This causes release of various lytic enzymes from lysosomes and PCD factors from mitochondria. C) Interactions at four VI 
loci, vic3, vic6, vicl and vic4 in C. parasitica are presented. The vic3 locus does not contain any HET domain genes. It consists of two polymorphic genes vic $3 a$ and vic $3 b$ separated by a homologous region that contains a gene coding for actin-binding-like protein. VI by vic3 is most likely triggered by nonallelic interaction between vic $3 a$ and $v i c 3 b$ from opposite strains. Similar to het-c in $N$. crassa, the vic6 locus provides an example of nonallelic interactions of linked genes. Here pix 6 is a small mobile protein that interacts with vic6 (HET domain) from incompatible strain to activate VI. The vicl gene cluster is a complex locus, involving at least 4 genes. Here, the allele 2 form (coloured orange) comprises two linked genes, vicla-2 and viclb-2, while the allele 1 form (coloured blue) contains vicla-1 and two additional ORFs, including viclc-1 and vicld-1, which encodes a HET domain. The precise role of viclc-1 and vicld-1 is not clear. Both allelic and nonallelic interactions involving vicla and viclb are believed to occur. A nonallelic interaction of nonhomologous genes occurs within the vic4 locus to trigger incompatibility. Here vic4-1 encodes protein kinase C-like protein and vic4-2 caries NACHT domain and WD40 repeat. Genes indicated in black are conserved, nonpolymorphic sequences that are not involved in VI but found linked to vic genes. 
genes are knocked out from both strains, leaving only the het-c genes, HI reaction does not occur when the two strains fuse (Figure I-3). Similarly, no HI reaction occurs when only functional pin-c genes are present. Thus, 'allelic' incompatibility is not exhibited by either of the het-c or pin-c genes. Another example from N. crassa of an incompatibility 'supergene' (i.e. tightly linked genes contributing to one incompatibility function (Cristina O. Micali and Smith 2006) are interactions at the het-6 locus. In this case, incompatibility is controlled by the tightly linked genes het-6 and un-24 (Smith et al. 2000). Where the two genes are locked in one of two haplotype forms (un- $24^{\mathrm{OR}}$ het- $6^{\mathrm{OR}}$ or $u n-24^{\mathrm{PA}}$ het- $6^{\mathrm{PA}}$ ) by a paracentric inversion and sequence divergence (Cristina O. Micali and Smith 2006). The un-24 gene encodes the large subunit of ribonucleotide reductase (RNRL), a component of the RNR holoenzyme that is essential for supplying deoxynucleotide triphosphates (dNTPs) for DNA synthesis and repair. In this case, incompatibility occurs through allelic interactions between $u n-24^{\mathrm{OR}}$ and $u n-24^{\mathrm{PA}}$ and through the nonallelic interactions between $u n-24^{\mathrm{OR}}$ and $h e t-6^{\mathrm{PA}}$ and between $u n-24^{\mathrm{PA}}$ and het- $\sigma^{\mathrm{OR}}$ (Lafontaine and Smith 2012). An interesting feature of het-6 incompatibility is the possible loss of RNR activity, encoded by the un- 24 gene, which may limit the dNTP pool and work as the trigger for PCD. Another well-characterized vegetative incompatibility factor in N. crassa is controlled by differences at the mating-type (mat) locus. $N$. crassa is the only known example where $\mathrm{HI}$ is triggered by opposite mating type genes during vegetative growth. Here, HI requires differences at the mat locus and a functional tol gene, which contains HET and LLR (leucine-rich repeat) domains (Shiu and Glass 1999). When tol is deleted, strains of opposite mating types are able to form viable heterokaryons. When $t o l$ is present, cells containing mat- $A$ and mat-a exhibit 
heterokaryon incompatibility during the vegetative phase of the life cycle. Involvement of un-24 (ribonucleotide reductase) and mat (mating type) present examples of incompatibility that is triggered by polymorphisms in genes involved in other basic cellular functions. Furthermore, mat presents an example of incompatibility that involves an unlinked, non-polymorphic HET domain gene (tol).

Another well-characterized incompatibility system is presented by Podospora anserina. For instance, the het-s locus appears to mediate incompatibility through prionlike interactions (Seuring et al. 2012). Here, when the prion protein HET-s occurs together with HET-S, it converts the latter into a toxic pore-forming fold. This is hypothesized to cause cell membrane disruption and eventually PCD (Riek and Saupe 2016). In the het-R/het-V system, two loci (het-R/het-r and het-V1/het-V) cause HI through non-allelic interactions. At the same time, het- $V$ is also involved in allelic incompatibility. A useful characteristic with this system is that when strains carry het- $R$ and het- $V$ alleles, temperature dependant incompatibility occurs (Pinan-Lucarré et al. 2007) whereby at $\sim 32{ }^{\circ} \mathrm{C}$ the strain grows normally but at $\sim 26{ }^{\circ} \mathrm{C}$ it becomes selfincompatible and undergoes PCD.

Genetic organization of C. parasitica VI loci is similar to what is found in these of other Ascomycota, N. crassa and P. anserina. In fact, based on het loci characteristics identified for $P$. anserina and $N$. crassa, together with genetic mapping studies (Cortesi and Milgroom 1998), it was possible to identify and characterize all six known vic loci in C. parasitica (G. H. Choi et al. 2012; D. X. Zhang et al. 2014) (Figure I-3c). It should be noted, in this regard, that there is evidence for additional vic loci in C. parasitica (Y.-C. Liu and Milgroom 2007). As seen in Figure I-3c, the vic3 locus of C. parasitica is 
comprised of two linked genes, vic $3 a$ and vic $3 b$, separated by a gene encoding an actinbinding-like protein. vic3-associated incompatibility is controlled by vic3a and vic3b genes (D. X. Zhang et al. 2014). It is not clear how these genes interact to cause VI, but by analogy with other known models this locus may involve nonallelic interactions, where smaller protein produced by vic $3 b$ may transfer to opposite strain and interact with vic $3 a$. Actin-binding-like protein is a nonpolymorphic gene that does not seem to play any role in incompatibility reaction (D. X. Zhang et al. 2014). Importantly, among the genes identified at the vic3 locus, none encode a HET domain. So, in addition to proposed nonallelic interactions, this system may involve one or more nonpolymorphic HET domain genes.

The organization of the vic6 locus in $C$. parasitica is very similar to that of the het-6 and het-c loci in N. crassa that is described above (G. H. Choi et al. 2012). Two interacting genes govern vic6 incompatibility through nonallelic interactions, one of which (the vic6 gene) encodes a HET domain protein. The PIX6 protein appears to be highly mobile and moves into the opposing cytoplasm to trigger incompatibility via interactions with the VIC6 protein (G. H. Choi et al. 2012). The vicl locus presents the most complex genetic structure among VI genes in C. parasitica (D. X. Zhang et al. 2014). Here, the vicl-1 allele comprises four protein coding regions. Where two, vicla and vicld-1, encode HET domain proteins and another, viclc-1, showed significant similarity to LTR retrotransposons. Opposite allele, vicl-2, contained vicla region in addition to another idiomorphic vic $1 b-2$ (Figure I-3c). In this locus, the most pronounced VI is determined by combined allelic interaction between two polymorphic vicla alleles and in some part by nonallelic interactions involving viclb-2. A strain with viclb-2 
deleted was only able to form a weak barrage. Finally, on Figure I-3c, vic4 locus presents a distinct example of exclusively idiomorphic gene interactions that activate VI in $C$. parasitica (G. H. Choi et al. 2012). The alternate forms of the vic4 locus encode completely different, nonhomologous proteins. The vic4-2 gene encodes NACHT and WD40 motifs, domains that are generally associated with VI in P. anserina (Paoletti et al. 2007) (see Figure I-4). The other allele, vic4-1, encodes a protein with a protein kinase Clike domain. Interestingly, neither vic4-1 nor vic4-2 genes carry the HET domain. In total, there are three out of the six characterized vic loci in C. parasitica - vic2, vic3, and vic4that do not have a tightly linked HET domain gene. These genes may, nevertheless, function similarly to VI loci in other fungi that require an unlinked, nonpolymorphic gene that encodes a HET domain protein. For example, the previously mentioned mat incompatibility in $N$. crassa requires a functional copy of tol, which encodes a protein containing a HET domain (Shiu and Glass 1999). Thus, hypothetically, a difference at one of the non-HET vic genes in C. parasitica may cause activation of other genes that encode HET-domain proteins to trigger VI, although these hypothetical interactions between vic loci and unlinked HET-domain loci have not been explored in C. parasitica.

The molecular function of HET domain genes is not clear and data on HI regulation is very fragmented. Nonetheless, studies of HET-domain genes in N. crassa have identified a transcription factor, vib-1, as an upstream regulator. So far, three HI systems have been identified to be under VIB-1 regulation. When vib-1 is knocked out, VI functions associated with mat, het-c and het-6 loci are altered (Dementhon et al. 2006; Lafontaine and Smith 2012). VIB-1 shows homology to Ndt80p, a p53-like transcription factor from yeast (Glass and Dementhon 2006; Xiang and Glass 2002). In yeast, Ndt80p 
is involved in several processes, including the regulation of sexual cycle, sporulation and meiosis (Chu and Herskowitz 1998). Ndt80p works in pair with Sum1p, a transcription repressor, which binds to the same promoter region as Ndt80p (Pierce et al. 2003). Analysis of all Ndt80p orthologs in N. crassa genome show that none of them influence meiosis, but mutations in these genes cause defects in related processes of sexual development, protoperithecia formation, and ascospore maturation (Hutchison and Glass 2010). In comparison to yeast, sexual reproduction in filamentous ascomycetes may be viewed as a more elaborate process, which involves the formation of specialized reproductive hyphae, sexual organs, and fruit-bodies. Regulatory proteins, once in control of simple reproduction of unicellular fungi, may have diverged in function to modulate organ regulation and other processes.

At this time, there are two general hypotheses concerning the origin and evolution of HI. First is the "accident" hypothesis, which posits that, due to increasing genetic polymorphism among isolated fungal stains, some gene allele combinations trigger PCD or other lethal reaction by chance when they occur in the same heterokaryon. A second hypothesis is that VI loci represent a subset of 'allorecognition' loci that are part of the innate immune system (Saupe 2000; Smith and Lafontaine 2013). The idea that VI represents a specific allorecognition system comes from the proposition that it evolved to increase the overall fitness of the individual. Some authors have proposed that fungi may gain an advantage by having VI systems that prevent 'infection' by more aggressive genotypes or viruses (Cortesi et al. 2001; Debets and Griffiths 1998; van Diepeningen et al. 1997; S. Wu et al. 2017). 
From the virulence perspective, and according to various studies, the main mode of action of VI is to activate secondary metabolites production, or more specifically, toxins (Leveau and Preston 2008; Wichmann et al. 2008). In support of the allorecognition hypothesis, some HET domain proteins are classified as members of the STAND protein family and show a resemblance to NOD-like receptors (NLR) (Paoletti and Saupe 2009) (Figure I-4). STAND proteins are employed in different functions in all types of organisms, including for apoptosis induction, active transport, and innate immunity (Leipe et al. 2004). STAND proteins in animals and plants are components of mechanisms that identify pathogens by detecting the presence of conserved cellular components. These systems incorporate pathogen-recognition receptors (PRRs), including NLR that use leucine-rich repeat (LLR) domains to identify specific pathogen elements (Leulier and Lemaitre 2008). In plants, for example, LLR at C-terminus of the protein recognizes patterns such as bacterial flagellin protein. At the N-terminus of the protein there could be CC (coiled-coil leucine zipper) or TIR (Toll/interleukin-1 (IL-1) receptor) domains, which are activated upon LLR interaction with target molecule and trigger a downstream immune response (Nürnberger et al. 2004). A main difference between plant and animal innate immunity factors is in the TLR N-terminal domain, which is CC or TIR in plants and CARD (caspase requirement domain), TIR or PYD (pyrin N-terminal homology domain) in animals (Paoletti and Saupe 2009). Unlike animals, plants are considered to have no adaptive immunity, but they maintain a larger set of LLR genes ( $\sim 235$ genes/genome), as compared to vertebrates ( $\sim 10$ genes/genome) (Nürnberger et al. 2004). As seen in Figure I-4 from general schematics, the HET 


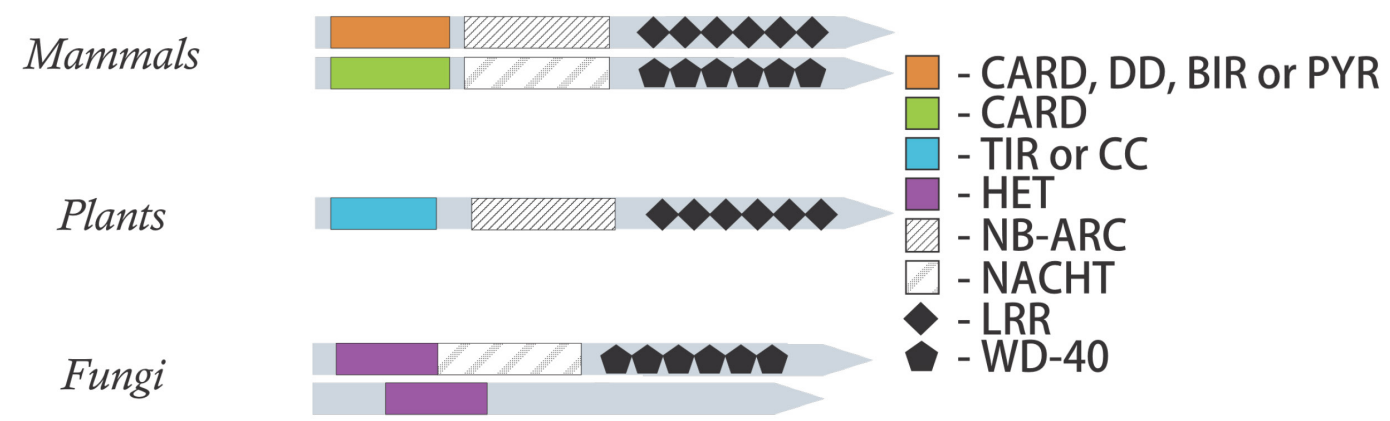

Figure I - 4. Generalized representation of proteins involved in innate nonself recognition in animals, plants and fungi. All these proteins activate $\mathrm{PCD}$, when trigged by incompatibility.

The only complex gene organization of VI loci in fungi is represented by HNWD family (top). This protein family is well studied in P. anserina, in which case HET domain serves as a PCD effector (Paoletti and Saupe 2009). Complex gene organization like in HNWD is rarely found in C. parasitica or N. crassa, where majority of VI loci incorporate only the conserved HET domain. Grey arrows indicate sequence direction from $\mathrm{N}$ - to C-terminus. 
domain, when found in multidomain genes, usually holds a N-terminal position. This may imply functional similarity to innate immunity genes, since most STAND proteins of that kind have an $\mathrm{N}$-terminal domain that is involved in cell death regulation (Paoletti and Saupe 2009). In this regard, fungi appear similar to plants in having a large number of HET-domain-encoding genes in the genome (usually over 50), which may indicate that they also lack an adaptive immune system.

Following an immune reaction trigger is induction of various defence mechanisms, many of which are associated with the production of toxic secondary metabolites and reactive oxygen species (ROS) (Fuchs and Mylonakis 2006). Such toxin production presumably evolved as a weapon against invaders, but may be lethal for the host fungus as well. For example, gliotoxins produced by Aspergillus spp. must be removed from the cell because high concentrations can lead to cell death (Owens et al. 2015). Indeed, vulnerability to endogenous toxins is sometimes used by parasites of fungi. For example, Pseudomonas syringae, a parasite of $N$. crassa, seemingly acquired a homolog of the $N$. crassa het-C gene, and upon infection, is able to trigger a reaction similar to VI (Wichmann et al. 2008). Why do fungi carry toxins that can kill them if activated? One possible explanation from the perspective of multicellular mycelial organism is that infection does not happen across the entire mycelium, but usually affects only a small portion. In this sense, a fungus that can produce a suicidal compound can sacrifice a small part of itself in an attempt to stop infection. As a result, the fungus separates the infected region from the rest of the mycelium, and toxins help to deter the pathogen from moving forward into other parts of the mycelium. Toxins, as part of the defence/offence system in fungi, are basically secondary metabolites, which through a 
long process of an evolutionary arms race become lethal for certain organisms. The evolutionary "purpose" of toxins is pretty blunt: to interfere with vital functions of a cell. This broad definition of purpose creates endless possibilities in defense, pathogen potential and ecological interactions. For the fungus, keeping a large reserve of toxin genes may be risky since accumulation of toxic potential creates a dangerous situation for the fungus itself. The more toxin that a fungus can produce, the more chances that they could be triggered by a random mutation in regulatory or other genes, or could be turned against them by another organism. Toxins must be activated only when needed, which means that fungi have to develop a genetic equilibrium to keep all the toxins silent or non-lethal to self as much as possible.

Both the 'accident' and 'allorecognition' hypotheses underline a similar end result for any incompatibility mechanism - programmed cell death (PCD). Various studies have shown that cell death during VI is morphologically similar to apoptosis, emphasising an active death mechanism rather than a 'failure to survive' associated with necrosis (Glass et al. 2000; Leslie and Zeller 1996). It should be noted that the allorecognition mechanism contains a significant gap in the proposed pathway. It proposes that a VI factor like HET-encoding gene triggers allorecognition and leads via a specific transduction pathway to an end result of PCD. Little is known about the putative intermediate signal transduction pathway linking recognition to death. On the other hand, the 'accident' hypothesis may incorporate a direct trigger of PCD. For example, an 'accidental incompatibility' may cause PCD directly through formation of a toxic protein complex, a failure of detoxification mechanisms or a disturbance of redox balance. However, considering the 'allorecognition' hypothesis may reveal pathways ionvolving 
toxin activators, het or vic genes. Furthermore, the 'accident' and 'allorecognition' hypotheses may not be mutually exclusive.

\section{Programmed cell death in fungi}

In animals, apoptosis is associated with development, response to infection and elimination of damaged cells. Since these functions may not be readily attributed to unicellular organisms, apoptosis was originally considered to be lacking in unicellular eukaryotes like the yeast, Saccharomyces cerevisiae. Nonetheless, apoptosis was eventually discovered in yeast and found to serve similar functions (Carmona-Gutierrez et al. 2010), although, here, the term "apoptosis" may not be an appropriate name for the process, as it does not include all the morphological features observed in mammals. Nonetheless, it is referred to as apoptosis in the sense that a yeast colony can be seen as a multicellular organism. By removing old cells, which can be then used as a nutrition source for new cells, apoptosis serves as the mechanism for maintaining colony homeostasis. The discovery of apoptosis in S. cerevisiae eventually made yeast a model for studying mammalian apoptotic proteins (Sato et al. 1994; Q. Xu and Reed 1998).

As for most organisms, apoptotic death in yeast acts as a reaction against an adverse environment. Apoptosis can be triggered by diverse factors such as reactive oxygen species (ROS), mycotoxins or bacteria. For example, high ROS concentrations are too damaging for the cell and usually cause necrosis, but at lower doses, $\mathrm{H}_{2} \mathrm{O}_{2}$ can cause an apoptotic reaction associated with activation of YCA1 and AIF1 (apoptosis inducing factor 1) (F. Madeo et al. 2002; Wissing et al. 2004). In addition, it was found that sexual reproduction in yeast includes PCD as one of the outcomes (Pozniakovsky et al. 2005; Severin and Hyman 2002; N. N. Zhang et al. 2006b). The presence of the 
opposite type of sexual pheromone causes ROS production and activates the mitochondrial branch of PCD. Activation of this PCD pathway is not dependant on cell age since chances of undergoing PCD are equal for mother and daughter cells $(\mathrm{N} . \mathrm{N}$. Zhang et al. 2006b).

In terms of molecular mechanisms, metazoan apoptosis can be subdivided into extrinsic and intrinsic. Both types involve a caspases cascade activation and regulators from the Bcl-2 family. With the extrinsic pathway, molecular signals for apoptosis come from outside the cell by activating the so-called 'death receptors'. These in turn pass a signal to caspases 8 or 10 . The classic apoptosis pathway - intrinsic - first found in $C$. elegans, presents the simplest model. Here, CED-9 -| CED-4 > CED-3 consecutive reaction leads to apoptotic death. To initiate the reaction, CED-9 is downregulated by the anti-apoptotic protein EGL-1, which belongs to the BCL-2 regulators family. Deactivation of CED-9 leads to the release of CED-4 adaptor protein, which in turn activates CED-3 (Metzstein et al. 1998). In mammals, the BCL-2 family includes CED orthologs, but here they activate downstream cascade of caspases. In this model, BCL-2 anti-apoptotic proteins protect the cell by inhibiting pro-apoptotic BAX/BAK proteins, also BCL-2 members. BAX and BAK reside within the mitochondrial membrane and cause damage by forming a channel upon activation (Scorrano and Korsmeyer 2003). This channel releases mitochondrial proteins like Cytochrome $c$, which lead to apoptosome formation. In animal models, apoptosome is formed by protein Apaf-1, a member of the STAND protein Ap family (Leipe et al. 2004; Riedl and Salvesen 2007). Apaf-1 consists of CARD (caspase recruitment domain) middle NB (nucleotide binding) and C-terminal WD40 repeats. Apoptosome formation starts with activation of Apaf-1 by 
Cytochrome $c$, which interacts with WD40 domains. The unlocked Apaf-1 forms a heptamer, where individual subunits are joined by the NB domain. The apoptosome activates Caspase-9, ortholog of CED-3, causing further activation of downstream caspases (Caspase-3, Caspase-6, Caspase-7), which induce the apoptosis program (Degterev et al. 2003; Mace and Riedl 2010).

In filamentous fungi, processes morphologically associated with PCD were found to be part of various functions such as aging, heterokaryon incompatibility, and spore formation (Glass et al. 2000; Raju and Perkins 2000; Sharon et al. 2009). One of the main obstacles in fungal PCD research is an identification of fungal apoptotic genes. Fungal apoptotic genes are not true homologs of mammalian genes, making it very hard to identify them by DNA or protein sequence comparisons. Fungal apoptotic genes usually have one domain (which is directly related to it's function), as compared to mammalian apoptotic genes that have several domains (Sharon et al. 2009). For example, in fungi, final stages of PCD are carried out by cysteine proteases that exhibit the activity of caspases (usually two or three per genome). They function as caspases in recognizing typical caspase substrates and show some level of homology to mammalian caspases. However, these fungal proteases lack the Cas domain, a signature component of mammalian and plant caspases. For that reason, these proteins are considered ancient versions of caspases and are termed metacaspases (Shlezinger et al. 2012; Tsiatsiani et al. 2011). Apart from metacaspases, some orthologs of mammalian apoptotic genes were identified in fungi. An ortholog of IAPs (Inhibitors of Apoptosis Proteins), Bir1p, is the only known example of an apoptosis inhibitor in yeast (Owsianowski et al. 2008). Similar IAPs were identified in filamentous fungi, like cpBirl in C. parasitica (K. Gao et al. 
2013). Interestingly, orthologs of apoptotic genes in filamentous fungi show more similarity to their mammalian versions than to the yeast forms (Shlezinger et al. 2012). Nonetheless, there no complete models of PCD pathways in filamentous fungi and at this time data remains fragmented.

\section{Cryphonectria parasitica as a model organism}

Cryphonectria parasitica, the causal agent of chestnut blight, was first identified in North America in 1904 (Merkel 1906). In the course of one human generation, the chestnut blight disease destroyed, by different estimates, three to four billion American Chestnut (Castanea dentata) trees (Anagnostakis 2001; Freinkel 2007; Roane et al. 1986). At about the time when the tree achieves sexual maturity $C$. parasitica forms cankers on the tree trunk, girdling and killing the tree. The tree regenerates from the root collar to repeat this cycle. As a result, what was once a dominant overstory tree, is now reduced to an understory shrub.

The discovery of virus-infected hypovirulent strains of $C$. parasitica in Europe opened a new page in the history of chestnut blight. From that moment on, C. parasitica was studied as a model of three organisms - tree, fungus and virus - a three layer hostparasite interaction. Hypovirulent strains of C. parasitica were first identified in Europe and then found in North America. These strains demonstrated altered colony morphology, manifesting as reduced growth rate, female sterility or inability to produce perithecia, reduced asexual sporulation and reduced pigmentation (Anagnostakis 1982; Nuss 1996). These strains also showed different traits on infected trees, usually called 'healing cankers'. Normally C. parasitica infect chestnut trees through damaged bark and 


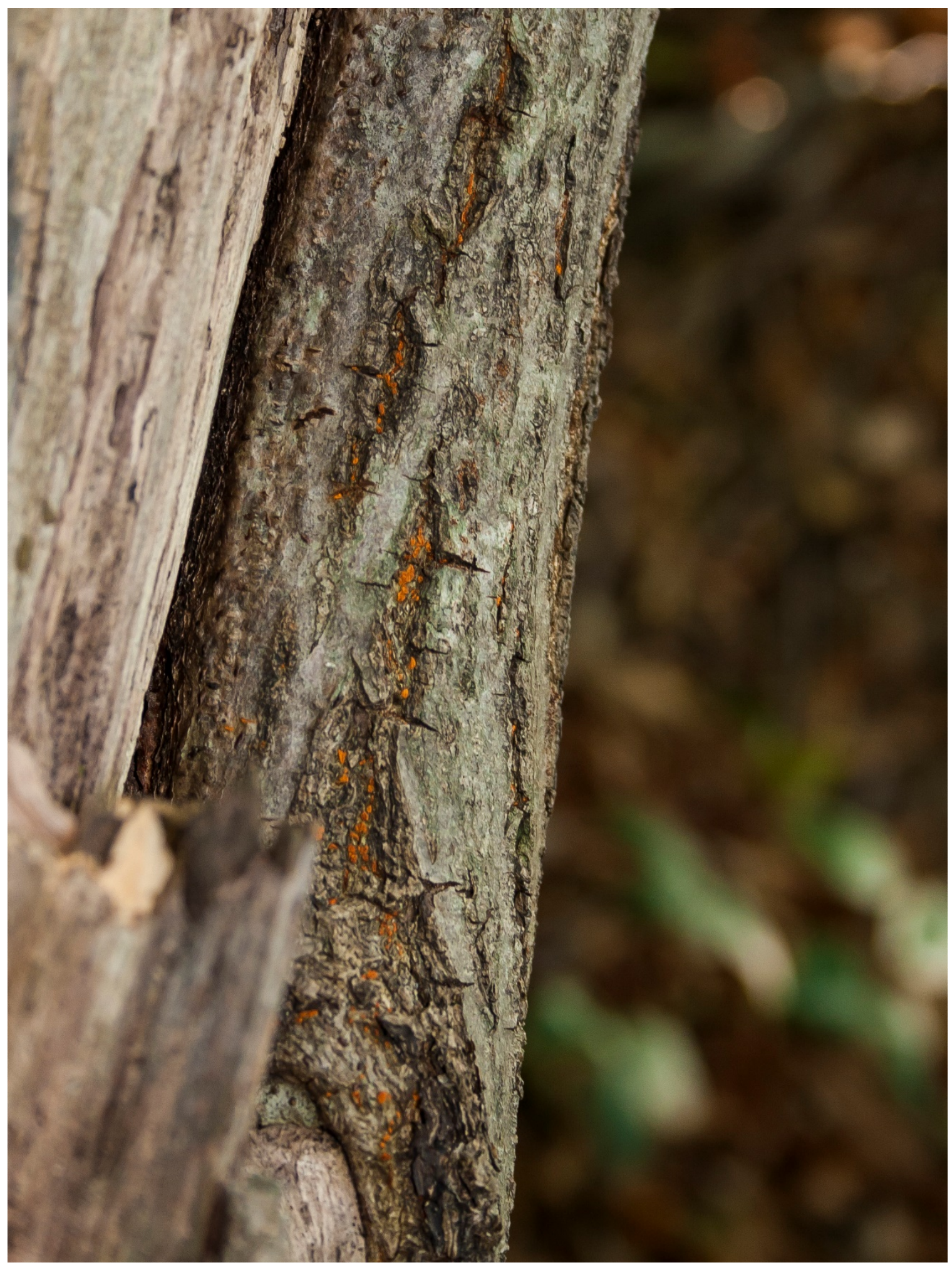

Figure I - 5. Canker on chestnut bark showing exposed C. parasitica conidia. 
spread throughout the cambium, manifesting as cankers on the surface of the bark (Figure I-5). Cankers function to expose $C$. parasitica stromata and perithecia to the outside environment. As the fungus grows under the bark, it encircles the tree stem blocking the flow of nutrients to leaves. Trees are usually infected at early developmental stages and die at about the time they reach the sexual reproductive stage. In contrast, when a tree is infected with a hypovirulent strain, cankers appear more localized and are surrounded by callus tissue, with no signs of orange conidia. In this situation, the tree is able to survive and reproduce.

C. parasitica can be infected by several types of mycoviruses (Hillman and Suzuki 2004). Hypovirulence symptoms, however, are caused by CHV1 (Chryphonectria hypovirus 1) (G. H. Choi et al. 1991). CHV1 is a typical example of a mycovirus; its genome is a double-stranded RNA (dsRNA) and it lacks a protective protein shell. Little is known, however, about the CHV1 life cycle. Some studies have shown that CHV1 stimulates production of vesicles, which encapsulate viral dsRNA, show polymerase activity, and p29 protein is harboured on the vesicle surface (Jacob-Wilk et al. 2006).

The absence of a protein shell makes it possible for CHV1 to spread only through hyphal anastomosis. Here, the subject of hyphal fusion and allorecognition becomes relevant again. As proposed previously, one of the main functions of barrage is to restrict the transmission of cytoplasmic genetic elements, like viruses (Cortesi et al. 2001; van Diepeningen et al. 1997). In the early years when hypovirulence of $C$. parasitica was discovered, researchers tried to use CHV1 as a biological control agent. The American population of $C$. parasitica, however, was found to be genetically diverse and contained a wide range incompatibility groups (Y.-C. Liu and Milgroom 1996). This created a natural 
barrier for CHV1 in North America. Further studies showed that different vic associated types of incompatibility allow different rates of virus transmission (Cortesi et al. 2001). For example, incompatibility by the vic4 locus causes a weak form of barrage and does not restrict $\mathrm{CHV} 1$ transmission at all (100\% transmission). On the other hand, vic7 differences result in a strong barrage, but very asymmetrical rates of CHV transmission depending on allele. If vic7-1 is a donor of the virus, transmission into a vic7-1 partner is $100 \%$. When vic $7-2$ is a donor, the rate drops to about $40 \%$ (Cortesi et al. 2001). Strains incompatible by vic3 locus showed symmetric transmission rates of $\sim 75 \%$ for both alleles. Importantly, studies have shown that the CHV1 transmission rate depends on the cell death rate during incompatible fusion (Biella et al. 2002).

Functional dissection of the CHV1 genome identified the relevance of each region to phenotypic changes associated with hypovirulence. The dsRNA genome of CHV1 consists of two open reading frames: ORF A and ORF B (Figure I-6). Several studies have demonstrated that major symptoms associated with hypovirulence are determined by proteins encoded in ORF A. Translated ORF A produces a p69 protein that undergoes autocatalysis into p29 and p40 proteins. Major hypovirulence symptoms, such as loss of pigmentation, lack of conidiation and female sterility, are determined by the p29 protein (Craven et al. 1993; Suzuki et al. 1999; Suzuki et al. 2003). The p29 protein shows significant homology to HC-Pro protease from plant potyviruses (Fukuzawa et al. 2010; Suzuki et al. 1999). Early studies also showed that deletion of p29 from the CHV1 genome does not impede its ability to replicate, but C. parasitica 


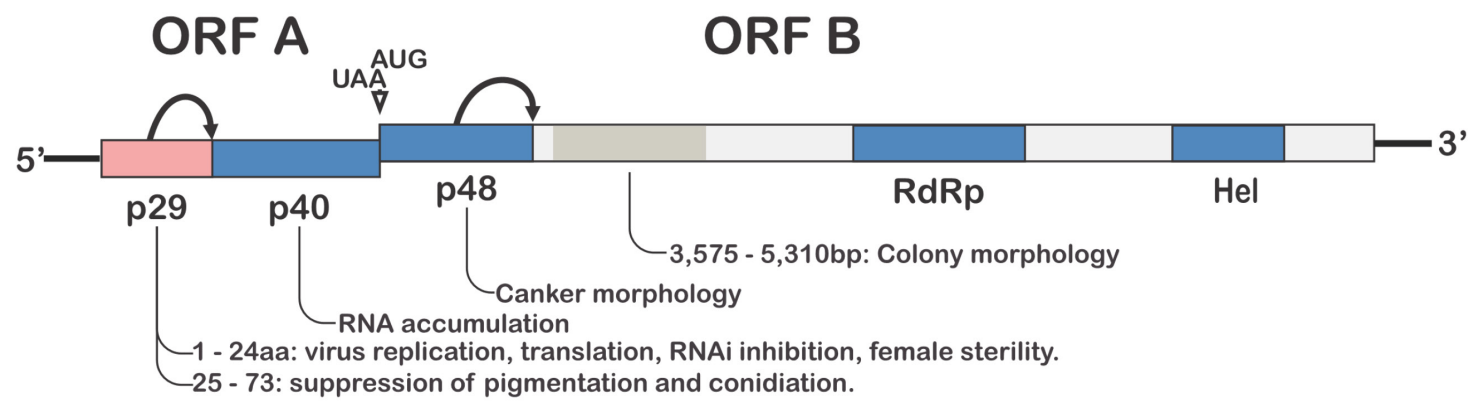

Figure I - 6. CHV1 genome organisation. Black arrows indicate autocatalysis point. White arrowhead shows junction site between two ORFs. RdRp indicates a region with RNA-dependent RNA polymerase domain; Hel - helicase domain. Text under genome indicates the regions that were associated with certain hypovirulence symptoms (Dawe and Nuss 2001; Segers et al. 2007). 
strains infected with CHV1 $\Delta$ p29 regain normal levels of pigmentation, asexual sporulation, and laccase activity (Craven et al. 1993). There is no complete picture about molecular function of p29, but recent findings indicate that p29, similarly to HC-Pro, serves as suppressor of RNA silencing (RNAi) (Fukuzawa et al. 2010; Segers et al. 2006; Segers et al. 2007). These studies show that among two C. parasitica Dicer proteins, only Dicer-2 is responsible for the defence against viral dsRNA (X. Zhang and Nuss 2008). Mechanics of RNAi machinery, on the other hand, require several proteins to form the RISC, a complex which uses small RNAs as guides to identify and cleave target RNA (Siomi and Siomi 2009). Apart from two Dicer genes, there were four Argonaute genes found in the $C$. parasitica genome. Further examination showed that antiviral RNAi requires a single Argonaute, agl2, to form RISC complex with Dicer-2 to work against viral dsRNA (Sun et al. 2009a). These studies also show that other Dicer and Argonaute genes are not activated in response to CHV1 dsRNA. These studies show that CHV1 can inhibit RNAi by downregulation of agl2 gene (Sun et al. 2009a) and inhibition of RNAi by CHV1 is mediated by the p29 protein, as its deletion causes normal activation of silencing (Segers et al. 2006; Segers et al. 2007). In light of our study model (discussed further in Chapter II), we can hypothesize that inactivation of $d c l 2$ or agl2, or the presence of p29 alone, can cause similar effects on fungal phenotype as infection with the CHV1 virus.

Other regions of the CHV1 genome can influence fungal phenotype, but in a limited and dependent manner (Dawe and Nuss 2001). Deletion of a second proteincoding region of ORF A, p40, results in a replication competent virus, but reduces its RNA accumulation (down to $\sim 50 \%$ ) (Suzuki and Nuss 2002). On ORF B, p48 protein 
showed significant homology to p29 and also appears to regulate pigmentation and conidiation (Deng and Nuss 2008). Interestingly, p48 deletion changed canker morphology, supressing pustule formation on canker face (B. Chen et al. 2000). Additional studies of several types of different CHV1-like viruses allowed for comparisons and further functional dissection of viral genomes. Using two viral strains, CHV1-EP713 (causes severe hypovirulence) and CHV1-Euro7 (mild hypovirulence), B. Chen et al. (2000) created chimeric forms exposing functional regions. Their results found that a region of about $1800 \mathrm{bp}$, right after $\mathrm{p} 48$ on ORF B is responsible for affecting colony morphology of the fungus (Figure I-6).

\section{Objectives}

In this thesis, Next Generation Sequencing (NGS) RNA-seq analysis was used to explore the transcriptional profile of $C$. parasitica strains undergoing barrage formation. For that purpose, strains incompatible by the vic3 locus (EP155 and P74-3) were used as they always demonstrate distinguishable barrage and symmetry in CHV1 transmission rates. As discussed above, CHV1 transmission depends on the rate of PCD during barrage. Thus, this study hypothesizes that CHV1 has evolved a mechanism to alter PCD to increase its own transmission. Also, previous studies have shown hypovirulence symptoms caused by CHV1 are determined primarily by the p29 protein. In this study, I used C. parasitica strain EP155p29 that has the DNA of the p29-encoding gene transformed into strain EP155 to simulate CHV1 infection. Additionally, to compare effects associated with p29, C. parasitica strains were used with mutated $d c l 2$ gene,

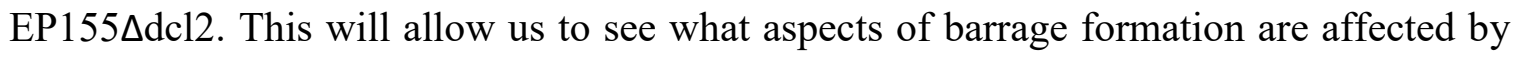


p29 and RNAi. Finally, to assess effects of p29 protein more effectively we created a strain, EP155p29stop. This strain, similarly to EP155p29, was transformed with p29 sequence from CHV1-EP713 but the p29 sequence contained three stop codons at the beginning of coding region. That way EP155p29stop strain can produce mRNA of p29, but not protein.

This thesis comprises three research chapters. In Chapter II, I present an analysis of RNA-seq data, describing changes in overall transcription profile caused by barrage formation. This allows me to indicate molecular mechanisms associated with barrage formation on the scale of entire transcription profile. In Chapter III, I analyse effects of p29 protein and p29 RNA, and deletion of Dicer-2 gene on the fungal transcriptome. The analysis aimed to identify attenuations of transcription profile from two possible perspectives: in control (monoculture) strains and in strains undergoing barrage formation. Analysis in Chapter IV derives from data presented in Chapters II and III, to build a detailed pathway of programmed cell death associated with vegetative incompatibility. It presents a closer look into identified and possible processes activated during barrage, with the goal of presenting a complete pathway. 


\section{CHAPTER II. \\ RNA-seq analysis of vic3-associated barrage formation}

\section{Introduction}

Hyphal fusion, or anastomosis, is important for growth and development of the fungal mycelial network. However, fusion between two genetically different fungal strains can lead to activation of allorecognition mechanisms that are triggered by heterokaryon incompatibility (HI) loci, which are known as vic (vegetative incompatibility) loci in $C$. parasitica (Cortesi and Milgroom 1998; Glass et al. 2000; Glass and Dementhon 2006; Smith and Lafontaine 2013). If two strains carry different alleles at one or more vic loci, the fusion cells will undergo programmed cell death (PCD).

PCD in filamentous fungi is involved in a variety of functions like aging, heterokaryon incompatibility and spore formation (Shlezinger et al. 2012). Studying PCD in fungi faces certain challenges, as genes involved in apoptosis in animals and plants share low or no homology to factors involved in fungal PCD (Sharon et al. 2009). Some important apoptotic genes, such as Bcl-2 for example, have not been identified in fungi although there do seem to be analogs that fulfil the same function (Carmona-Gutierrez et al. 2010; Sato et al. 1994). Nonetheless, basic apoptotic genes like caspases (metacaspases in fungal nomenclature) and Apoptosis Inducing Factors (AIFs) have been identified in yeast studies, which reveals that apoptosis in fungi shares similarities with the intrinsic pathway of apoptosis in animal models (Carmona-Gutierrez et al. 2010; Tsiatsiani et al. 2011). In relation to allorecognition-associated PCD, the available data is limited. Studies on N. crassa showed that during heterokaryon incompatibility (HI), cells 
demonstrate all morphological features of apoptotic death (Glass et al. 2000; Glass and Dementhon 2006). Little can be said about the underlying molecular processes, except that they work through non-canonical processes that do not necessarily require caspases and AIF (Hutchison et al. 2009).

While PCD during nonself recognition in filamentous fungi demonstrates morphological features of apoptotic death (Glass and Kaneko 2003) (Dementhon et al. 2006), on a molecular level the mechanisms of this type of PCD type are not clearly identified. Among the known common features, incompatibility reactions in all studied Ascomycota involves HET domain genes (Glass et al. 2000) (Paoletti et al. 2007). When two strains with polymorphic HET genes fuse, these genetic differences trigger a cascade of reactions that lead to cell death (Glass and Kaneko 2003). In many ways, PCD during incompatibility resembles basic defence reactions during contacts with other organisms such as plants, bacteria, or other fungi (Lam et al. 2001). Also, several studies show that PCD during HI is associated with sexual and asexual spores formation. For example, PCD results in ascospore elimination in Coniochaeta tetrasperma, where only four out of eight spores develop (Raju and Perkins 2000). Similarly, a surprising effect was observed when Bcl-2 proteins were ectopically expressed in the plant pathogen Colletotrichum gloeosporioides (Barhoom and Sharon 2007). Here, expression of the anti-apoptotic Bcl2 protein increased lifespan of the mycelium and arrested conidia formation. However, when pro-apoptotic Bax was active, it largely reduced lifespan but increased conidia production. These studies indeed reveal that the roles of PCD in filamentous fungi are analogous to those in other organisms, but the lack of genetic homology between apoptotic genes makes it hard to dissect pathways. 
Another way to investigate PCD during incompatibility is to use transcription profiles to identify molecular processes associated with $\mathrm{HI}$ (heterokaryon incompatibility) (Bidard et al. 2013) (Hutchison et al. 2009). These studies showed that cell death during HI is most likely a caspase independent type of PCD (Hutchison et al. 2009). As well, it involves activation of a large number of HET genes and proteolytic processes.

In this study, we analyse transcription profiles of barraging and non-barraging $C$. parasitica strains. We provide general observations on overall expression patterns using principal component analysis (PCA) and identify genes that are differentially expressed during vic3-associated barrage. Functional annotations using various gene databases and enrichment analyses are performed on differentially expressed genes in order to understand the underlying mechanisms involved in allorecognition.

\section{Materials and methods}

Strains and growth conditions. C. parasitica strains P74-3, EP155, EP155p29, EP155p29stop and EP155 $\Delta$ dc12 were used in this study. Strain P74-3 and EP155 are of distinct genetic background but carry identical alleles at all vic loci except for vic3. EP155-derived strains carry the vic3-1 haplotype and P74-3 carry the vic3-2 haplotype.

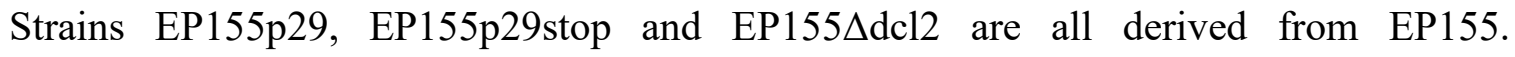
EP155p29 carries the coding region of p29 protein from CHV1-EP713 hypovirus under gpd1 promoter (G. H. Choi and Nuss 1992; Craven et al. 1993). EP155p29stop also carries the p29 sequence under gpd1 promoter, but has three stop codons inserted at the 5 '-end of the p29 coding region. As a result this strain produces p29 mRNA but not the 


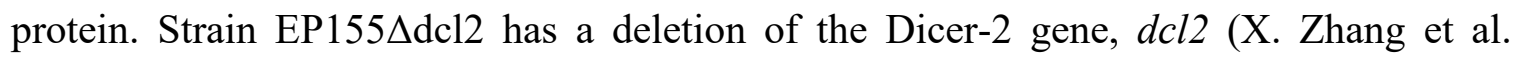
2008).

For obtaining barraging and non-barraging cultures we used a modified spheroplasting protocol by Churchill et al. (1990). Strains were grown in potato dextrose broth (PDB; Becton, Dickinson and Company, Sparks, MD) for two weeks at $30^{\circ} \mathrm{C}$ in the dark. The medium was then removed and mycelium was incubated with Trichoderma lysing enzymes ( $0.2 \mathrm{~g}$ of enzyme per $1 \mathrm{~g}$ of mycelium; Sigma-Aldrich Canada, Oakville, Ontario) in osmotic buffer (1.2M MgSO4, $10 \mathrm{mM}$ sodium phosphate, $\mathrm{pH} 5.8)$ for 1.5 hours at $30{ }^{\circ} \mathrm{C}$. Hyphal fragments/spheroplasts were then washed twice in Trapping Buffer (0.4M D-Sorbitol; 100mM Tris-HCl, $\mathrm{pH} 7.0)$ and centrifuged at 1500g. Washed hyphal fragments/spheroplasts were resuspended in $400 \mu \mathrm{l}$ of 1M D-Sorbitol and kept at $30^{\circ} \mathrm{C}$ in the dark for 6 hours. To obtain a large number of barrage interactions, approximately equal colony forming units (CFUs, assessed by optical density) of vic3incompatible strains (P74-3 + EP155-derived strains) were mixed and placed onto the surface of sterile cellophane overlaid on top of potato dextrose agar (PDA) medium in Petri plates. For control self-pairings (monocultures) equivalent CFUs of each strain were separately plated onto cellophane on PDA. Plates were incubated at $30^{\circ} \mathrm{C}$ without light.

To determine the optimal time point for RNA extractions, we monitored vic3associated PCD using Evans Blue and fluorescent microscopy. An aqueous solution of Evans Blue dissolved in $100 \mu \mathrm{l}$ of PDB making final $0.001 \%$ solution was applied directly to $1 \mathrm{~cm}^{2}$ of cellophane containing barraging or non-barraging hyphae and viewed under fluorescent light (Carl Zeiss AxioVision microspore, red filter excitation and green filter emission). Evans Blue accumulates in dead and dying cells. Area measurements of 
fluorescence intensity were made on days 2, 3 and 4 after plating hyphal fragments. To evaluate death rate we made several images under fluorescent light in random areas of slide. Images were then converted to high contrast, so that bright fluorescent areas become white and the rest of the image black. The number of fluorescent (white) pixels was measured on the image as a relative measure of death rate. Area of fluorescent staining, as a measure of cells undergoing PCD frequency peaked on days 2 - 3 . Preliminary assessments indicated that RNA yields dropped dramatically from day 3 to day 4. An optimal time to monitor vic3 incompatibility reaction was thereby determined to be 3 days after mixing hyphal fragments and plating.

RNA preparation and analysis. Total RNA was extracted on day 3 of growth on PDA plates with the Plant-Fungi RNA extraction kit (Norgen Biotek, Thorold, Ontario, Canada). RNA quality was assessed with Agilent Bioanalyser (Santa Clara, CA, United States) and final concentration was adjusted to $1500 \mathrm{ng}$ per sample. RNA sequencing was done on Illumina NextSeq platform, with paired-read length 150 bp and $40 \mathrm{M}$ read depth (StemCore Laboratories, Ottawa, Canada).

RNA-seq data analysis. Short reads quality was assessed with FastQC and reference based alignment was performed with TopHat2 (Kim et al. 2013). Reference genome and annotation were obtained from the $C$. parasitica genome project (http://genome.jgi.doe.gov/Crypa2/Crypa2.home.html). Additionally, novel transcripts from regions of the genome that showed significant expression, but were not previously annotated as genes were identified using Cufflinks v.2.2.1 (Pollier et al. 2013; Trapnell et al. 2012). Transcripts were identified by estimation of FPKM (Fragments Per Kilobase of transcript per Million mapped reads) using alignment data obtained from TopHat2. 
Identified transcripts showing FPKM $>10$ were considered as expressed. Differential expression analysis was done with R package DESeq2 (Love et al. 2014) and further data analysis and visualisation was done in the R environment. Gene Enrichment analysis was done using DAVID v6.7 (Huang et al. 2009; Huang da et al. 2009). Categories of GO biological processes and INTERPRO conserved domains were grouped by DAVID based on the heuristic fuzzy partition algorithm, where distances between genes are estimated with kappa statistic (for details on each GO terms group see Table S2).

\section{Results and Discussion}

In this section I examined the $C$. parasitica transcriptome response to allorecognition in vic3-incompatible strain pairings (P74-3 + EP155) in comparison to self-pairings. A previous study indicated that vic3-associated nonself recognition is influenced by the hypovirus element p29 from CHV1-EP713 and by defects in RNAi (Biella et al. 2002; Craven et al. 1993; Sun et al. 2009a; Tanha 2008). Therefore, we examined possible alterations in gene expression patterns under barraging and non-barraging conditions

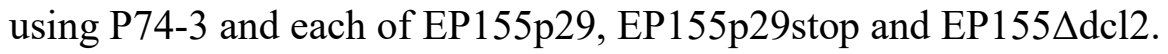

\section{Death rate and barrage formation.}

Barrage formation is a very rapid, but localised process. In standard barrage tests incompatible strains are co-inoculated $1-10 \mathrm{~mm}$ apart and allowed to grow together over a period of days. The barrage forms as a line along the confluence of the two colonies that covers less than about $1 \%$ of total colony areas (Figure II-1a, left). To overcome of the limitation of having relatively few interacting cells, we used small mycelial fragments, 

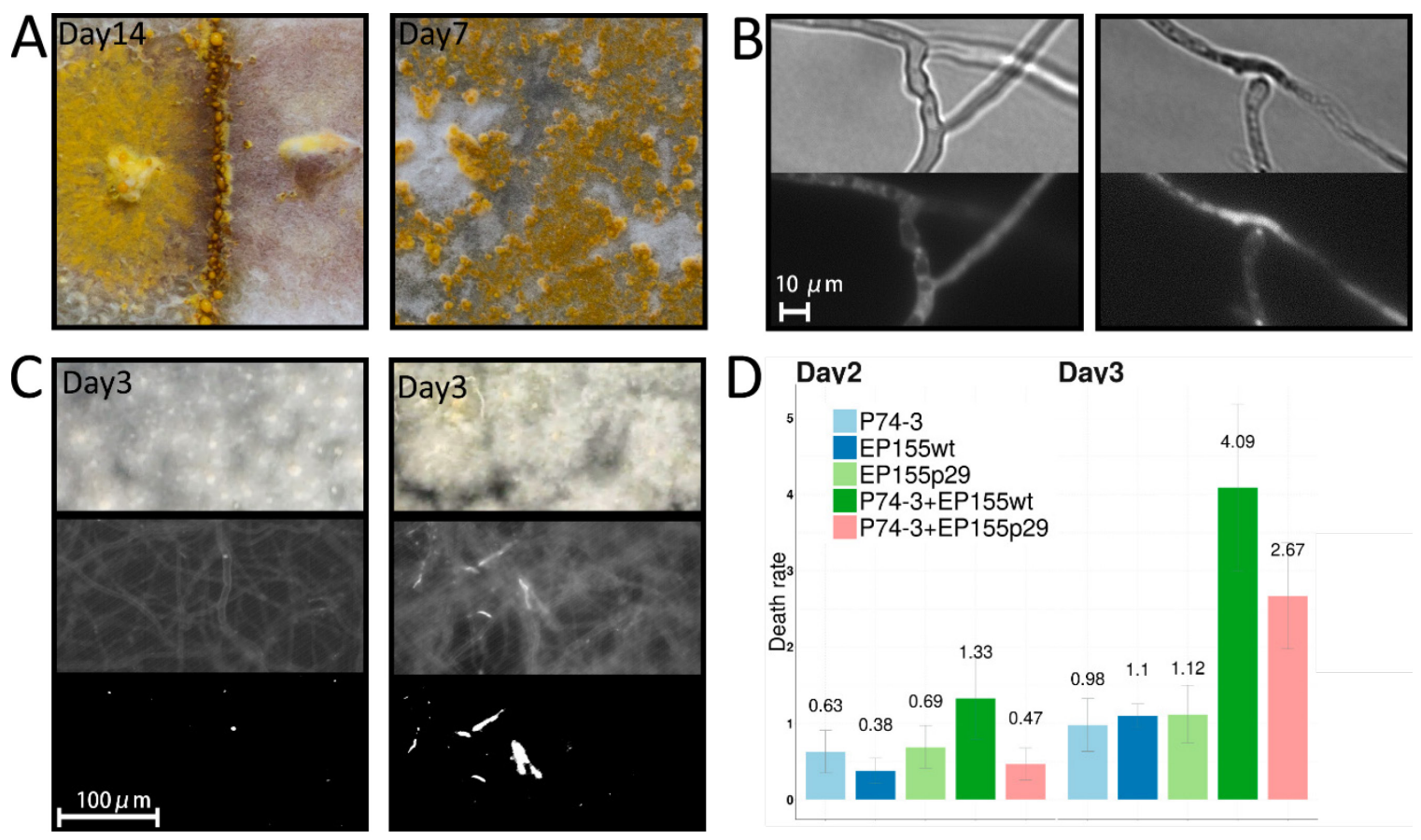

Figure II - 1. Barrage formation and programmed cell death during vic3 incompatibility. A) Barrage between two strains (left) is formed by a narrow line of dying cells and represents a small portion of total mycelial area. To increase the number and surface area of barrage we lysed (with Trichoderma lysing enzyme) mycelium to create hyphal fragments of two incompatible strains, mixed and plated them on PDA medium (right) creating an even distribution of interacting cells. B) Interactions between compatible (left) and incompatible (right) hyphae stained with Evans Blue dye. Shown in ambient light (top) and under fluorescent light (bottom). Bright fluorescence of incompatible interaction (bottom right) indicates dead and dying cells. C) An assay used to estimate relative death rate. Fragments of plated lysed hyphae were stained with Evans Blue and photographed. Top images show growing mycelia on PDA plates (no magnification). In the middle microscopy images show hyphae under fluorescent light and on the bottom are the same fluorescent images after contrast adjustment. Compatible strains in monoculture (left) demonstrate very little fluorescence compared to mix of incompatible strains (right). D) Death rate estimation using fluorescence assay with Evans Blue staining. Data collected for controls in monoculture and mixed cultures of incompatible strains at days 2 and 3 after plating. Numbers represent relative values of area having bright fluorescence (Evans blue stained) calculated using images similar to ones shown on bottom part of panel C. Grey bars indicate standard error $(n=5)$. 
mixed in equal proportion and inoculated on PDA (Figure II-1a, right). This technique created an even distribution of barraging cells over the entire surface area. It should be noted, however, that mixed in with nonself interactions are hyphal contacts and fusions between the same strain (self-interactions), and that contacts, anastomoses, and onset of PCD are not expected to be synchronous.

To obtain an idea of optimal timing to examine the barrage process, we used fluorescent microscopy and Evans Blue staining (Figure II-1b). This dye accumulates in dead and dying cells and is pumped out of healthy cells. This method allowed us to estimate the relative proportions of cells undergoing PCD on a large sample area over a period of time. Differences in Evans Blue fluorescence in barraging mixtures compared to controls becomes evident from day 2 and peaked on day 3 after inoculation. Unfortunately, after day 3 the mycelium becomes too thick for accurate measurements using this method. To evaluate death rate using fluorescent images we used an algorithm that initially brings the picture to a maximum contrast, turning all pixels to white or black. Following this, the number of white pixels remaining on the image is counted (Figure II1c). This allows us to make an approximate measure of the number of dead cells in the focal plane of the slide. There are many available algorithms designed for yeast and bacteria that can select single cells and detect fluorescence. However, as evident from Figure II- $1 \mathrm{c}$, in the case of filamentous fungi, it is very problematic to single out individual cells. For that reason, we preferred to use estimates of total area of dead cells. Final estimation results are shown on Figure II-1d.

Analysis of RNA extractions performed 2, 3 and 4 days after inoculation allowed us to fine-tune our approach. Quality estimations of extracted samples made at these three 
Table II - 1. Spectrophotometry analysis of total RNA extracted from barraging and control samples.

Barraging strains (right) combined P74-3 with each of the strains listed.

\begin{tabular}{|c|c|c|c|c|c|c|c|c|c|c|}
\hline & & \multicolumn{5}{|c|}{ Controls strains } & \multicolumn{4}{|c|}{ Barraging strains } \\
\hline & Strain & P74-3 & EP155 & $\begin{array}{c}\text { EP155 } \\
\text { p29 }\end{array}$ & $\begin{array}{c}\text { EP155 } \\
\text { p29Stop }\end{array}$ & $\begin{array}{l}\text { EP155 } \\
\Delta \mathrm{dcl} 2\end{array}$ & EP155 & $\begin{array}{c}\text { EP155 } \\
\text { p29 }\end{array}$ & $\begin{array}{c}\text { EP155 } \\
\text { p29Stop }\end{array}$ & $\begin{array}{l}\text { EP155 } \\
\Delta \mathrm{dcl} 2\end{array}$ \\
\hline \multirow{3}{*}{ day 2} & RNA ng/ $\mu \mathrm{l}$ & 509 & 384 & 412 & 345 & 378 & 339 & 408 & 506 & 432 \\
\hline & $260 / 280$ & 2.18 & 2.15 & 2.13 & 2.16 & 2.15 & 2.14 & 2.13 & 2.17 & 2.14 \\
\hline & $260 / 230$ & 2.30 & 2.23 & 2.20 & 2.21 & 2.24 & 2.17 & 2.15 & 2.24 & 2.24 \\
\hline \multirow{3}{*}{ day 3} & RNA ng/ $\mu \mathrm{l}$ & 153 & 143 & 136 & 97 & 326 & 215 & 72 & 223 & 188 \\
\hline & $260 / 280$ & 2.08 & 2.12 & 2.13 & 2.11 & 2.16 & 2.13 & 2.04 & 2.15 & 2.14 \\
\hline & $260 / 230$ & 2.18 & 2.02 & 2.05 & 1.84 & 2.26 & 2.10 & 1.80 & 2.20 & 2.15 \\
\hline
\end{tabular}


time points demonstrated rapid degradation of RNA after the $3^{\text {rd }}$ day (data not shown).

The amount of RNA ( $\mu \mathrm{g} / \mathrm{mg}$ tissue) from some samples extracted on the $3^{\text {rd }}$ day were about 3-5 times lower than from samples taken on day 2 (Table II-1). Based on the above, we carried out transcriptome analyses on barraging and control (self) cultures on day 3 after inoculation.

\section{RNA-seq data evaluation}

Sequenced RNA aligned to reference EP155 genome was further analysed at the gene level by differential expression (DE) analysis. Using R package DESeq2 (Love et al. 2014), we estimated gene DE by subtracting normalised expression for each gene in control samples (means of P74-3 and EP155-derived strain values) from the corresponding values from barrage samples (mixed cultures of P74-3 + EP155-derived strains). In all subsequent data analysis, genes that are upregulated in barrage samples will have positive DE values and downregulated genes will have negative DE values. Functional annotation and gene mapping was acquired from the JGI $C$. parasitica genome portal version 2 which contains information about 11610 C. parasitica protein coding areas with associated GO terms and INTERPRO domains (http://genome.jgi.doe.gov/Crypa2/Crypa2.home.html). To see if there are other transcriptionally active regions outside of acquired annotation we performed novel transcript discovery with Cufflinks2 (Trapnell et al. 2012). Using RNA-seq alignment data, Cufflinks2 maps transcriptionally active regions by estimating FPKM (Fragments Per Kilobase of transcript per Million mapped reads) for each region. In combination with existing annotation it allowed us to identify 2334 novel transcriptionally active $($ FPKM $>10)$ areas of the genome in addition to those previously 

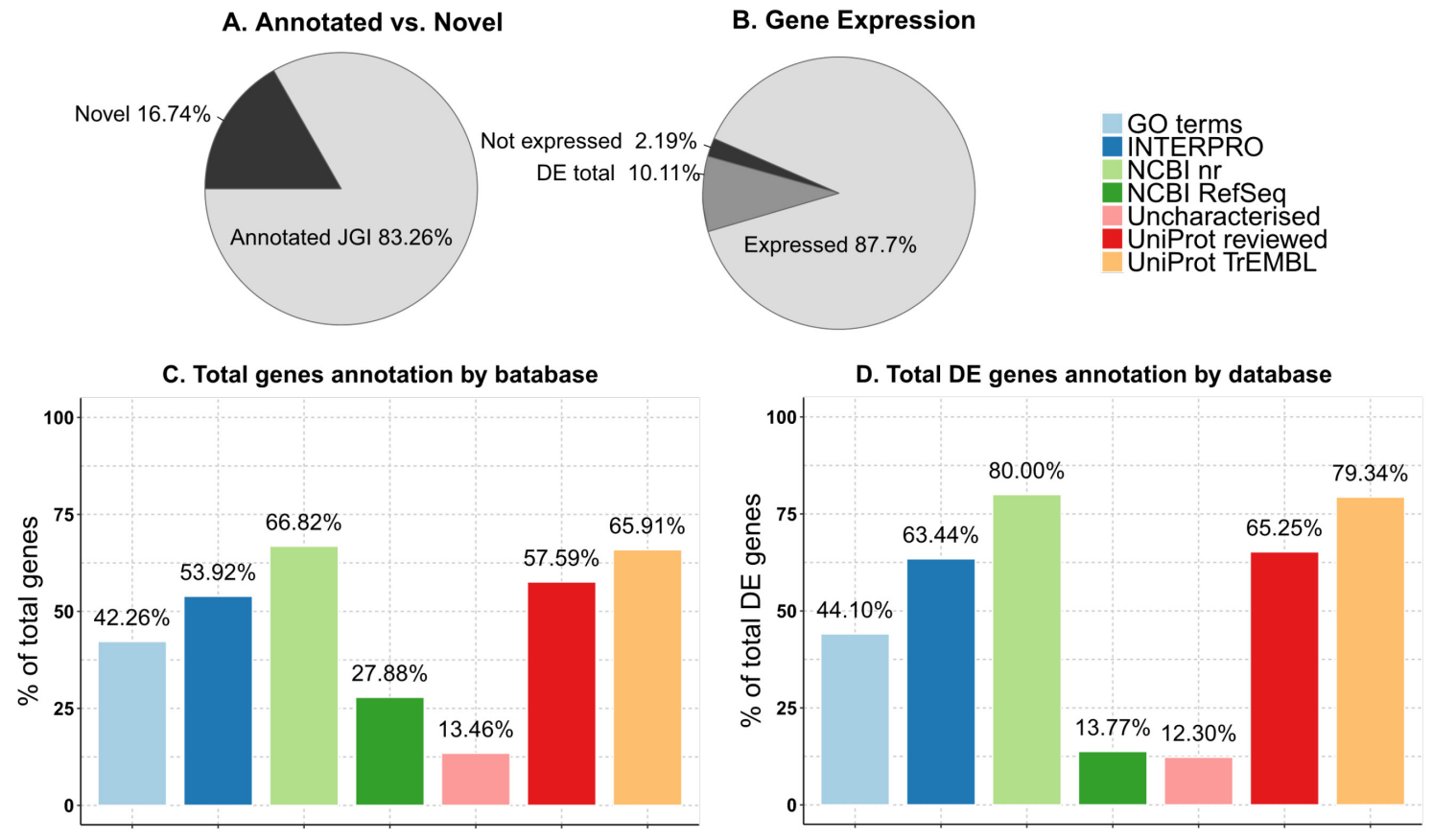

Figure II - 2. Annotation data of $C$. parasitica genome, where genes previously identified and novel transcripts were annotated with BLAST $+\left(\mathrm{e}-\mathrm{value}<10^{-10}\right)$.

Following databases were used: GeneBank and UniProt (with associated INTERPRO domain ids). Annotations available at JGI (C. parasitica genome project version 2, http://genome.jgi.doe.gov/Crypa2/Crypa2.home.html) were used to assign C. parasitica protein ids with associated GO terms and INTERPRO domains. A) Proportions of novel genes identified in this study with Cufflinks 2.2.1 transcriptome annotation tool (Trapnell et al. 2012) in addition to all previously annotated genes in genome project. B) The proportions of detected expression for all annotated and novel genes. DE Total percentage of differentially expressed genes in barraging strains out of total genes. Not expressed - percentage of genome project annotated genes with no significant (FPKM < 10) transcript accumulation and not included in DE Total. C) Percentage of total genes from transcriptome datasets that are annotated with BLAST + in individual databases (evalue $\left.<10^{-10}\right)$. D) Percentage of DE genes annotated with BLAST $+\left(\right.$ e-value $\left.<10^{-10}\right)$ in total number of DE genes. Uncharacterised genes are ones with no significant (e-value $>$ $10^{-10}$ ) homologs in databases used in this study. 
annotated in $C$. parasitica genome v.2. These novel transcripts account for about $16 \%$ of the total (13944) annotated genes (Figure II-2a). RNA-seq alignment data additionally demonstrated that only a small group of $305(2.19 \%)$ annotated genes show no significant $($ FPKM $<10)$ transcript accumulation (Figure II-2b).

Using BLAST+ analysis we assigned UniProt and NCBI GeneBank IDs to all identified genes and associated INTERPRO/Pfam domains (Figure II-2c,d). The most coverage for homologous sequences was obtained from NCBI nr (nonredundant), UniProt TrEMBL (unreviewed, nonredundant database) and UniProt reviewed (SwissProt). Of these, the UniProt reviewed database provided the greatest amount of information, as needed for further functional analysis, and was used for subsequent enrichment analysis and functional characterization.

\section{Principal component analysis and Functional annotation}

Principal components analysis (PCA) shown on Figure II-3 reveals striking differences in overall gene expression patterns between control strains and those undergoing vic3-associated barrage formation. Here, the first two principal components, PC1 and PC2, account for $78 \%$ and $13 \%$ of total variance of the sample set, respectively. Control and barraging strains form two distinct clusters, with relatively small differences in gene expression variance within each. Also, expression patterns of EP155 $\mathrm{dcl} 2$ control and P74-3+EP155 ddc12 notably stand out along PC2 from other control and barrage combinations, respectively, the common feature being the $d c l 2$ knock-out strain. We infer that disruption of RNAi in EP155 $\mathrm{del} 2$ strain has a pleiotropic effect on the C. parasitica transcriptome. Further, expression patterns in self-pairings of P74-3 can be differentiated from self-pairings of EP155-derived strains in the control group (Figure II-3, blue dots). 


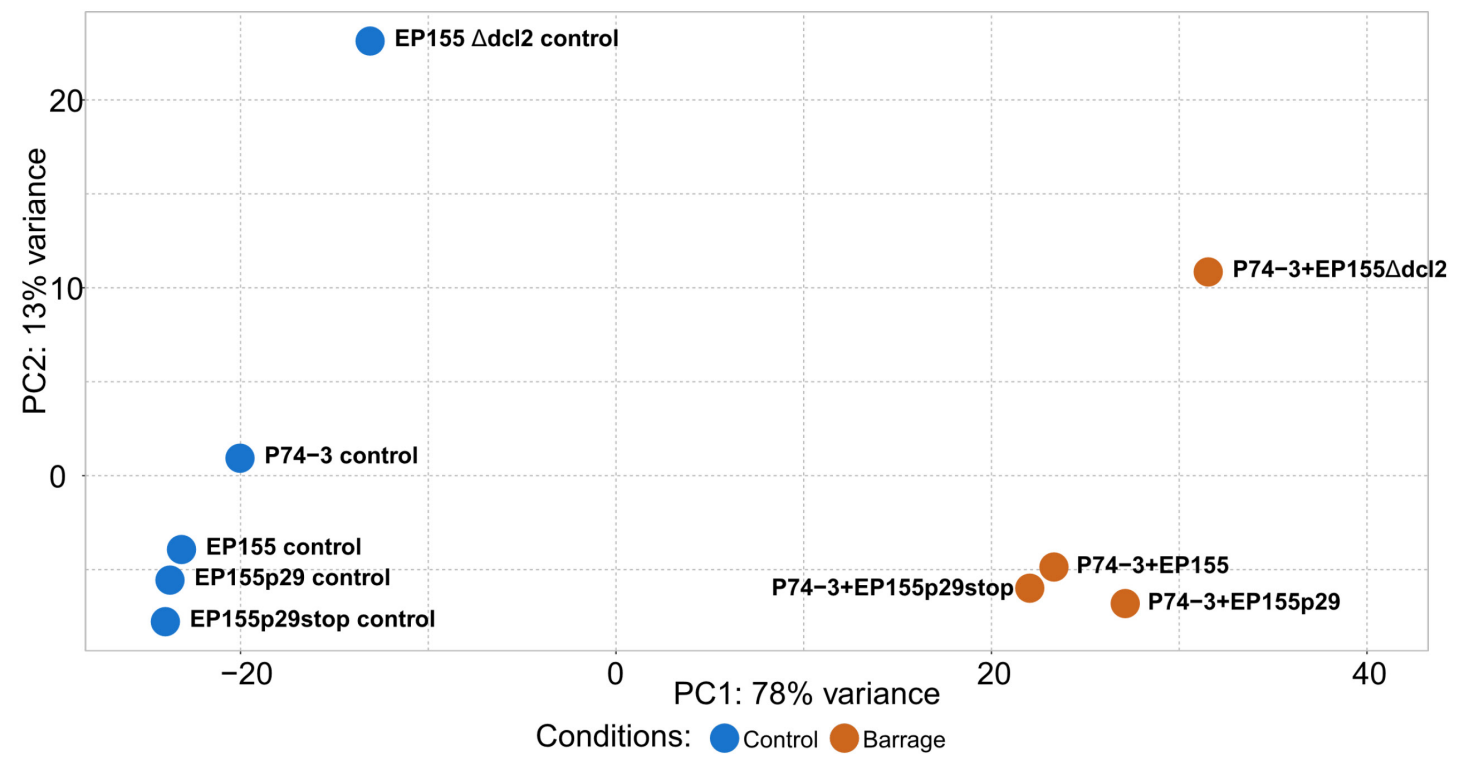

Figure II - 3. Principal components analysis (PCA) of normalised expression variance for intra-strain (blue dots) and inter-strain combinations (brown dots).

PCA and expression normalization were performed with DESeq2 R package. Principle Components 1 and 2 (PC1 and PC2) account for $78 \%$ and $13 \%$ of total variance, respectively. 
This difference was taken into account for differential expression analysis, as each sample in a barraging group represents a mix of wild-type P74-3 strain and one of the EP155-derived strains. To account for variations between EP155-derived strains and P743, we used mean expression values of P74-3 and the appropriate EP155-derived strain as the control expression value. Also, evident in Figure II-3, in the group of barraging strains, P74-3+EP155p29 is separated from P74-3+EP155 and P74-3+EP155p29stop along PC1. From this we infer that expression of p29 protein in the EP155 strain alters expression patterns during barrage, in comparison to barrages where no p29 protein is expressed, specifically those involving EP155 or EP155p29stop.

In the following two sections, we demonstrate the effects of barrage formation on gene expression patterns using hierarchical clustering and gene enrichment techniques. To do this, we compared gene expression values from pairings of vic3-incompatible strains to the mean expression values from the respective control strains in monoculture to estimate differential expression (DE) of each gene (Figure II-7). Overall, 1411 genes showed differential expression that was above threshold (log2 fold change $>2)$ in at least one pairing (i.e. P74-3+EP155p29, P74-3+EP155 and/or P74-3+EP155p29stop). These DE genes were used for further analyses of transcriptional effects of barrage formation. Furthermore, we used this data to analyse effects of CHV1 protein p29 and Dicer-2 deletion on gene expression during barrage formation. 


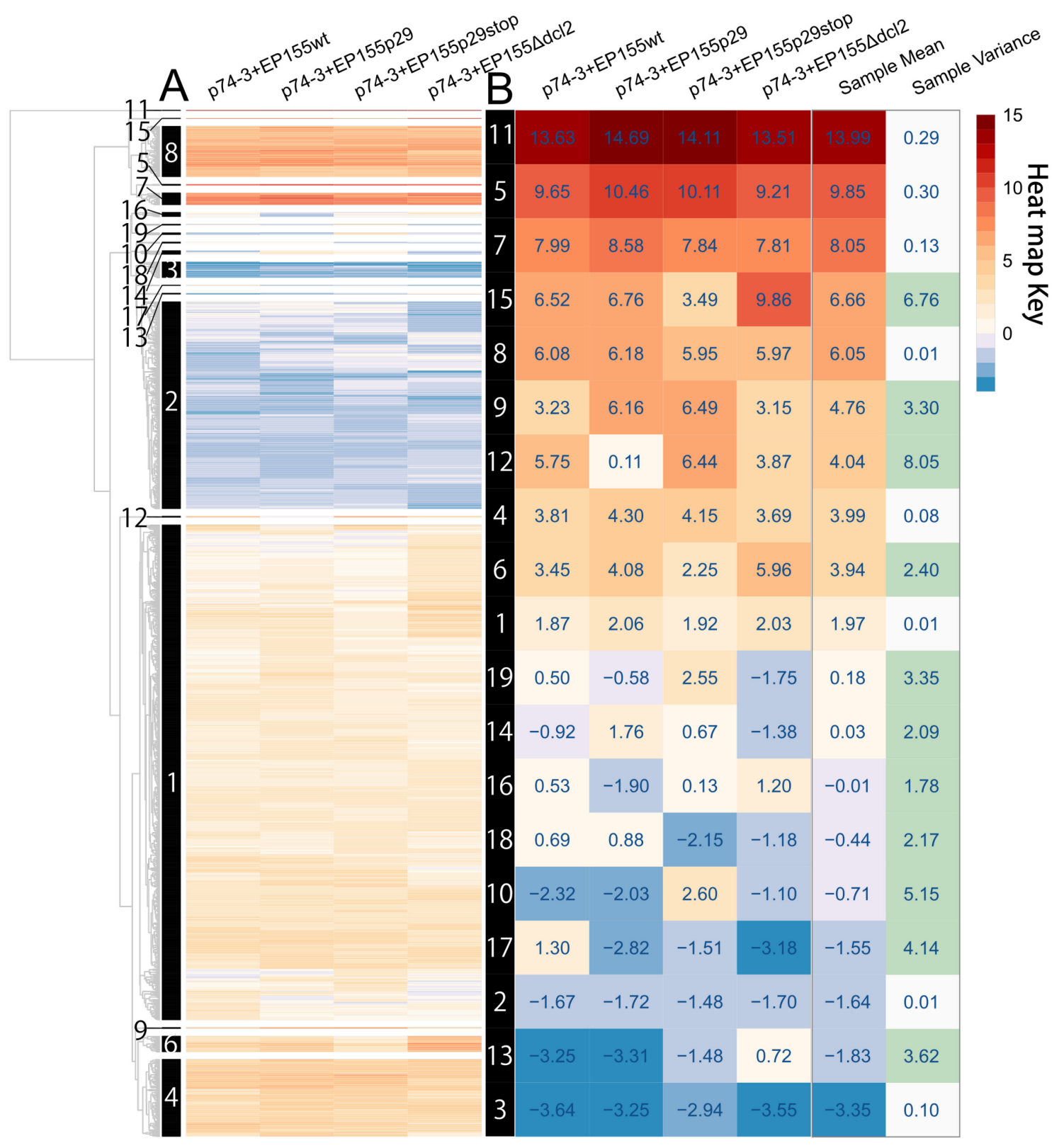

Figure II - 4. Hierarchical clustering of 1411 differentially expressed genes $(\log 2 \mathrm{FC}>2$, p-value $<0.0001)$.

A) Estimated Euclidian distance between expression values was clustered with UPGMA (Unweighted Pair Group Method with Arithmetic Mean). B) Mean expression for each sample in individual clusters. Heat map was arranged according to mean expression in individual clusters. Sample variance numbers highlighted green indicate values above threshold (variance $>1$ ). 


\section{Gene expression hierarchical clustering}

Hierarchical clustering of 1411 genes by their differential expression as shown in Figure II-4 allowed us to identify 19 robust clusters. From this cluster analysis, we can see that the majority of DE genes (74\%) are upregulated and occupy 12 clusters. Further, upregulation of many genes can be dramatic, with mean DE values well above threshold ( $\log 2$ fold change $>2$ ). Downregulated genes present a much smaller group that also tend to have minor differences in expression where many DE values are very close to the threshold (Figure II-4b).

The degree of variance within each cluster (right column of Figure II-4b) is useful in understanding allorecognition pathways. For example, eight clusters that showed relatively uniform expression (variance $<1.0$ ) across samples comprise $96 \%$ of DE genes. This uniformity among sample can serve as indicator that these genes are part of major processes activated during barrage formation regardless of the EP155 genotype. If we

take mean expression values into consideration along with uniformity, Clusters 5, 7 and 11 show uniform and high DE values (Figure II-4b). Remarkably, within Clusters 5 and 11 are three genes that have the highest expression rates in the entire sample set; these are the pheromone precursor genes $m f 2-1$ and $m f 2-2$, and the ortholog of $m t r$ gene from $N$. crassa that encodes an aliphatic and aromatic amino acid transporter (Dillon and Stadler 1994).

As I proceed with gene functional enrichment analysis I will use information from Figure II-4 to align functional and expression data by using the mean DE value and variance of a cluster. Clusters with high DE evidently represent an activated process during barrage, like Clusters 11, 5, 7, 15, 8 and 9. On the other hand, Clusters 1 and 2 
present DE genes which just made it over threshold $(>2)$, making activation or deactivation of any processes related to genes in this group inconclusive. Information on DE variance between samples will be especially useful in Chapter III, where I discus effects of ectopically expressed p29 protein on barrage formation.

\section{Gene expression enrichment analysis}

In this section I analyse DE genes enrichment by associated Gene Ontology (GO) terms and INTERPRO/pfam conserved domains using DAVID v6.7 (Huang et al. 2009; Huang da et al. 2009). The analysis is presented as combined observations from the hierarchical clustering and the enrichment analyses. This means that specific genes identified in enrichment groups are also discussed in context of the cluster they belong to

within Figure II-4. The gene enrichment accounts for functional annotation and the hierarchical clustering uses DE values, bringing these together helps to create a meaningful image of processes during barrage. This approach resulted in identification of at least four major processes occurring in barraging strains. These are: secondary metabolites biosynthesis, including mycotoxins; activation of detoxification mechanisms, most probably in response to toxins; sporulation; vegetative growth.

The gene enrichment analysis included two main elements: a list of DE genes for individual samples and a background list, against which enrichment of GO terms and protein domains was estimated. For that purpose, we used only genes that were annotated with UniProt reviewed IDs, as they have better functional annotation. A total number of 8031 C. parasitica gene IDs with UniProt annotation were used as a background list. For this analysis I used Gene Ontology (GO) terms and INTERPRO/Pfam conserved domains annotations to form gene enrichment groups (Figure II-5). 
Figure II - 5. (next page) Gene enrichment analysis of GO terms for biological process (A) and for INTERPRO/Pfam domains (B).

Groups in each diagram represent similar GO terms and INTERPRO/Pfam IDs grouped together by DAVID using Kappa statistic. Group definitions (names) were assigned based on types of GO terms and INTERPRO/Pfam IDs in each group. For more details see text and Table S2. 
A

Toxin biosynthesis

Regulation of Apoptosis

Tissue Morphogenesis/Growth

Sexual Reproduction/Sporulation

Pigment Biosynthesis/Sporulation

Cell Wall Development/Growth

RNA interference

Aminoacids Biosynthesis

DNA Repair/Res. to Abiotic Factor

Cellular Ion Homeostasis

Glycolysis

Sterol Metabolism

Amino Acid Transport

Peptide Transport

Alkaloid/ROS generation

DNA Repair/Res. to Abiotic Factor

Carbohydrate Biosynthesis

Coenzyme Biosynthesis

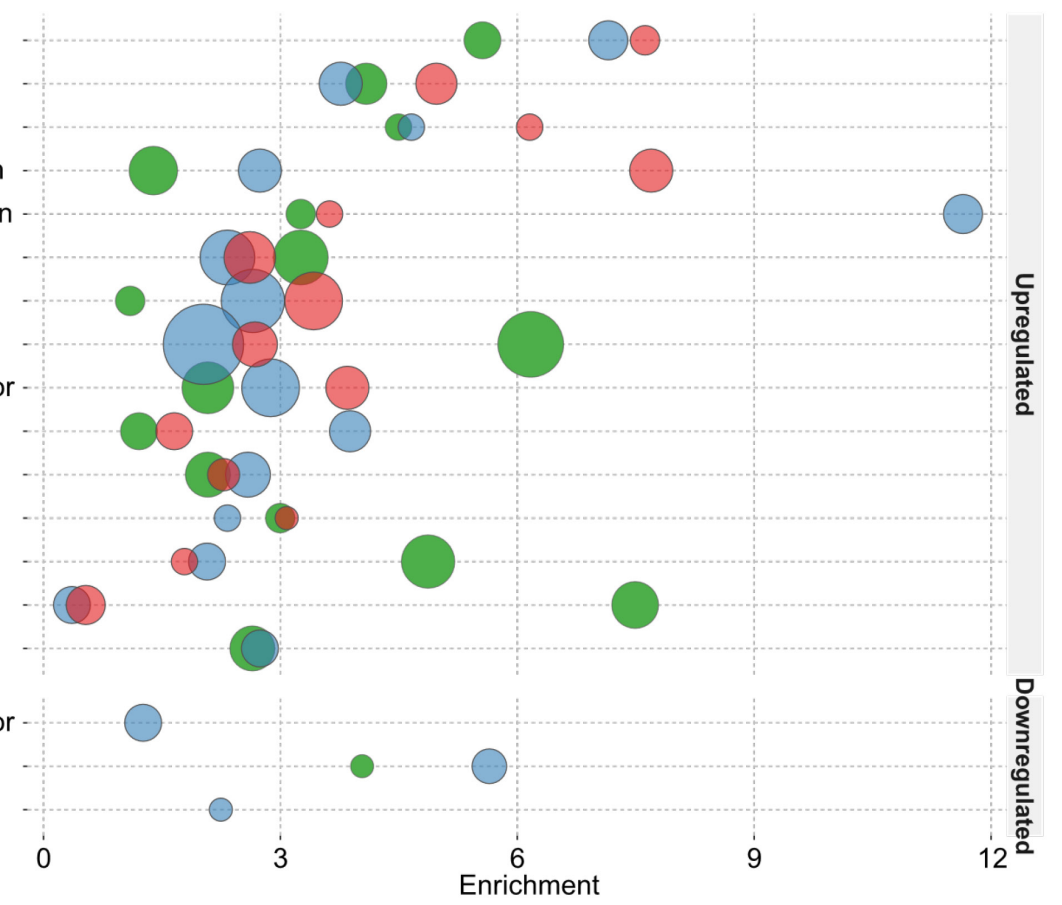

B

Cytochrome P450

$N A D(P)$-binding/dehydrogenase

Alcohol dehydrogenase

MFS transporter superfamily

Fatty acids/antibiotic synthesis

Amino acid permease

Sugar transporter

NmrA

TIM barrel fold

FAD binding domain

Glutathione S-transferase

Carboxylesterase

Cytochrome P450

Sugar transporter

MFS transporter superfamily

Glycoside hydrolase

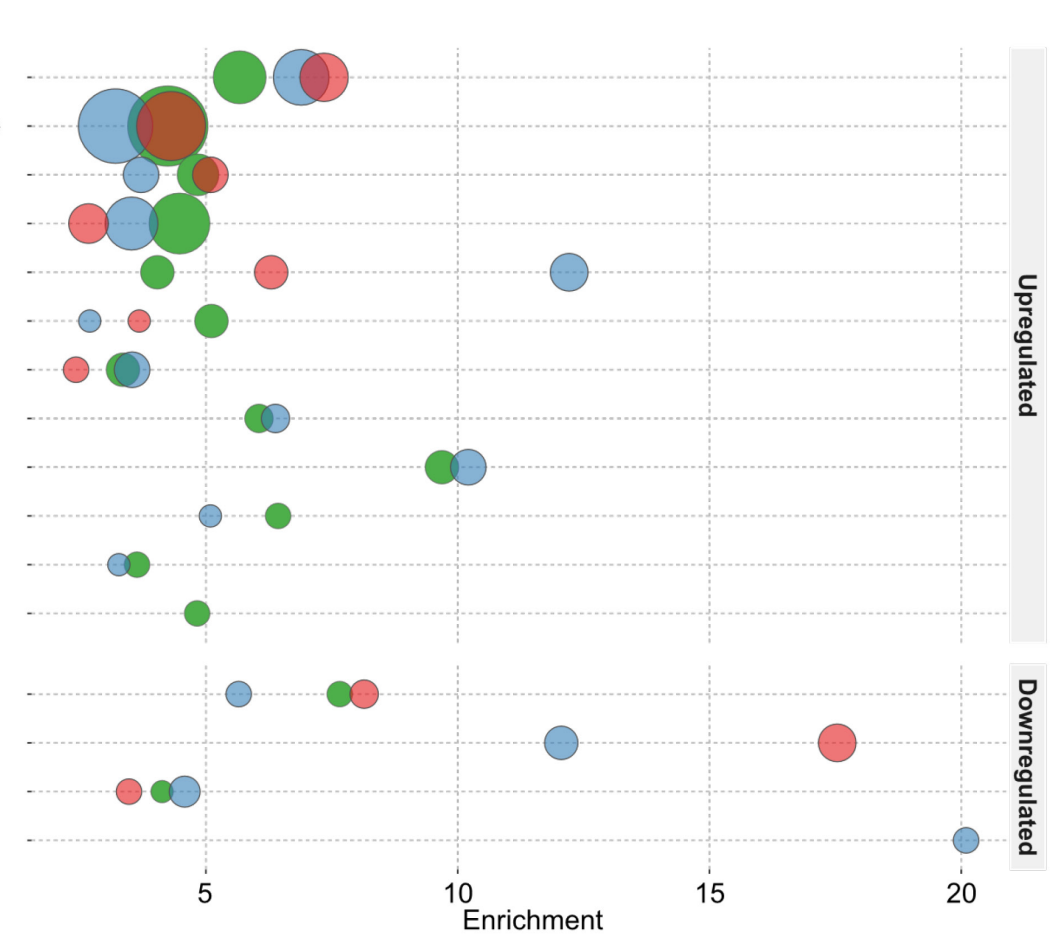

\section{Samples}

Number of genes

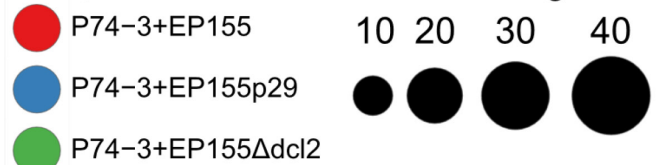




\section{Gene ontology enrichment analysis}

Gene ontology (GO) term enrichment analysis allowed us to identify the main groups of biological processes involved in allorecognition. Figure II-5a shows groups of associated GO terms listed by significance of their overall enrichment from top to bottom (for detailed groups' description see Table S2). This ranking system gathers together related GO terms, but does not reflect the number of genes associated with each GO term.

"Toxin biosynthesis" is the most enriched group, even though it includes a relatively small number of GO terms that include orthologs of Aspergillus genes activated during different phases of toxin biosynthesis. Five identified UniProt IDs include an ortholog of ftmD from Aspergillus fumigatus, a methyltransferase that is crucial in biosynthesis of Tryprostatin A (TPS-A), which causes phase specific cell cycle arrest (Kato et al. 2013; Usui et al. 1998). In C. parasitica genome two genes show homology to ftmD from Aspergillus fumigatus (proteinIDs 354223, upregulated; 255335, downregulated) that demonstrate drastically different expression behaviours during barrage (Table S1). In hierarchical clustering analysis, gene 255335 falls into the downregulated Cluster 2, whereas gene 354223 can be found in a highly variant and upregulated Cluster 9 (see Figure II-4b). Gene 354223 shows significantly higher DE rates in pairings with EP155p29 and EP155p29stop ( $6 \log 2$ fold) compared to wild-type ( $\sim \log 2$ fold $)$ and Dicer mutant $(\sim 2.5 \log 2$ fold $)$ pairings. Finally, the last three UniProt IDs in this group identify homologs of P450 monooxygenases from Aspergillus that regulate Sterigmatocystin biosynthesis. In C. parasitica genome there are a number of paralogs of these genes: monooxygenase $s t c F$ has three paralogs (proteinIDs: 346479 , 339678, 291163), stcS has two paralogs (proteinIDs: 269861, 342071) and stcC has one 
ortholog (proteinID: 18749). Four out of six of these monooxygenases are uniformly upregulated during vic3-incompatibility and all reside in Cluster 1 (see Figure II-4). In contrast, two genes, the $s t c C$ and the $s t c F$ (346479), are downregulated and found within Clusters 2 and 3. This peculiar up- and down regulation of functionally similar genes is also evident in Figure II-5b at the level of functional domain analysis, where the P450 domain (all Sterigmatocystin genes mentioned above contain cytochrome p450 domain) is seen as enriched among differentially regulated genes.

The second most enriched group includes GO terms associated with positive and negative regulation of apoptosis. Of interest among these genes are homologs of caspases (IDs: 66954, 260952). The first of these genes (66954) shows very weak similarity to human Caspase-9, and the second is one of the four predicted C. parasitica metacaspases (Tsiatsiani et al. 2011). As a hallmark of classic apoptosis pathway, the caspase cascade is activated primarily by post-translational modifications an so increased accumulation of transcripts is not an expected outcome (Riedl and Salvesen 2007). Accordingly, previous studies on $N$. crassa showed no caspase differential expression during HI (Hutchison et al. 2009). Further, on closer examination, C. parasitica gene 66954 has only minor homology to human Caspase-9 and for gene 260952 the metacaspase activity has not been confirmed. Therefore, whether these genes actually function as caspases is unclear. In addition, the other three $C$. parasitica metacaspases show no significant change in expression during vic3-assiciated incompatibility.

In general terms apoptosis in animals was subdivided into two categories: intrinsic and extrinsic. Intrinsic apoptosis is activated as result of internal regulatory signals. These signals can be activated due to processes like stress, ageing or high 
mutation rates (Shlezinger et al. 2012). This pathway is associated with cytochrome $c$ released from mitochondria that, in cooperation with Caspase 9, form a structure called the apoptosome (Riedl and Salvesen 2007). Extrinsic pathways may involve other caspases or be caspase independent (Tait and Green 2008). Here, apoptosis inducing factor (AIF) presents a well-studied example of caspase independent extrinsic pathway, which by being released from mitochondria activates PCD (Lorenzo et al. 1999). Of interest is the gene encoding the C. parasitica ortholog of mouse Aifm2 (ID: 285678), which shows significant overexpression in barraging samples. Aifm2 in mouse, also known as $A M I D$, is also upregulated during programmed cell death. In mammalian cells AIFM2 is activated by P53 as part of the apoptotic death response, playing a main role in DNA degradation. AIFM2 encodes a mitochondrial protein that is transcribed in the nucleolus, translated in cytosol and then transported to the mitochondrion (Modjtahedi et al. 2006). AIFM2 apoptotic function starts when it is released from mitochondria and moves back to the nucleus (Modjtahedi et al. 2006). The importance of AIFs for apoptosis is under debate and may vary from species to species, but in most cases reduced expression of AIF leads to deficiencies in apoptosis (Mei et al. 2006). In the context of our study, increased expression of AIF may serve as an indicator of increased apoptotic-like functions in association with (a) cell death activation by external signals and (b) mitochondrial membrane permeabilization (Modjtahedi et al. 2006; M. Wu et al. 2002).

Other examples of differentially regulated positive regulators of cell death include gene 75073. This gene is an ortholog of Protein roadkill $(r d x)$, which plays a role in an eye development in the fruitfly. Similar to AIF, $r d x$ orthologs are involved in the extrinsic 
cell death pathway, as they are known to activate the JNK-dependant apoptotic cycle (J. Liu et al. 2009).

Orthologs of negative regulators of apoptosis were also identified in "Regulation of Apoptosis" group (Figure II-5a). For example, an ortholog of human quinone oxidoreductase (ID:333952), which is upregulated during vic3-associated barrage. In animals, quinone oxidoreductases are part of the cell defence mechanism that is activated in response to abiotic stress factors to maintain the cell's redox balance (Johnson et al. 2008). Another GO term for negative regulation of apoptosis is represented by upregulated ortholog of human DHCR24 (3beta-hydroxysterol delta24-reductase, ID: 259069). In humans, this gene is a part of the cholesterol biosynthesis pathway and determines resistance to apoptosis caused by abiotic stress. Importantly, increased expression of DHCR24 leads to more resistance to apoptosis caused by ROS and other abiotic factors (Di Stasi et al. 2005; Kuehnle et al. 2008).

Overall, "Regulation of Apoptosis" GO group shows a definite picture of activated PCD processes. Here, barraging cells are most likely candidates to present positive regulators of apoptosis. In this case positive regulators present some evidence of extrinsic apoptotic cycles associated with JNK and AIF. Negative apoptosis regulation, on the other hand, probably comes as a defence reaction of surrounding cells.

One of the distinguishing features of the barrage in C. parasitica is bright orange coloration, associated with conidiophores along the zone of interaction between incompatible strains (see Figure II-1a). In this context, it is interesting to investigate the composition of GO group identified as "Pigment biosynthesis" (Figure II-5a). Unfortunately, among genes associated with "Pigment biosynthesis" group, only one 
shows as a possible candidate involved in production of orange pigment. Gene 337117 is an ortholog of Phytoene desaturase, al-1, which is a part of well-studied $\beta$-carotene biosynthetic pathway in N. crassa (Hausmann and Sandmann 2000). This gene is universally expressed during vic3-associated incompatibility and found in Cluster 4 (Figure II-4) and may be one of the contributors to conidia coloration. Activation of the al-1 ortholog during barrage suggests that a similar metabolic pathway in C. parasitica is involved in conidia pigmentation.

Other genes associated with GO terms joined under a "Pigment biosynthesis" group show significant similarity to ones involved in carotenoid and retinol biosynthetic pathways in bacteria and animals. These demonstrate no direct link to known fungal metabolic pathways. However, more detailed analysis with BLAST+ (less strict parameters were applied) allowed us to identify orthologs from filamentous fungi, most of which appear to be a part of toxin biosynthetic pathways. For example, gene 263100 appears as an ortholog of $\operatorname{crt} C$ (hydroxyneurosporene dehydrogenase). In bacteria, $\operatorname{crt} C$ is involved in synthesis of carotenoids, as a part of bacterial photosynthetic apparatus (Albrecht et al. 1997). However, among filamentous fungi, 263100 has sequence similarity to two equally homologous genes, $\operatorname{ccs} F$ (cytochalasins biosynthesis; UniPort id: A1CLZ1) and asqI (aspoquinolone biosynthesis; UniPort id: Q5AR52). The first belongs to a cluster of genes producing cytochalasins, including cytochalasin E produced by $A$. clavatus that is used as an anti-antigenic agent (Qiao et al. 2011). Others, like cytochalasin A and B, suppress glucose transport in humans (Rampal et al. 1980). The second, asqI, belongs to a gene cluster in A. nidulans involved in production of quinolone 
alkaloids (Ishikawa et al. 2014). This may indicate that 263100 contributes to toxin biosynthesis and/or pigment synthesis in the barrage zone.

GO term groups such as "Pigment biosynthesis", "Mycotoxin biosynthesis" and "Sporulation" notably overlap in their gene content. Each gene involved in biosynthesis of secondary metabolites can participate in production of various chemicals which can be classified under these different GO terms. A good example is presented by C. parasitica protein IDs 32824, 86830, 59909, 60079, and 292843, which are all homologs of polyketide synthase, wA, from Aspergillus. The wA protein is a part of naphthopyrene biosynthesis pathway, which is the yellow pigment expressed in Aspergillus conidia (Mayorga and Timberlake 1992). Two of the C. parasitica genes $(60079,292843)$ fall into the downregulated Cluster 2 (see Figure II-4), while the rest $(32824,86830,59909)$ are upregulated. These genes are associated with the enriched GO terms sporulation, pigmentation and sexual reproduction, but according to literature polyketide synthases are involved in synthesis of several secondary metabolites including mycotoxins (Fujii et al. 2001). In terms of protein domains analysis, polyketide synthases, including wA homologs, appear in a group of proteins with fatty acid synthase domains that includes several homologs from Aspergillus involved in small peptide antibiotics production. For instance, the Aspergillus proteins NRPS14 and NRPS8 (orthologs to C. parasitica IDs 338852 and 10611, respectively) have a wA domain composition, a nonribosomal peptide synthetase function and are involved in synthesis of various mycotoxins (Maiya et al. 2007). Considering the above, activation of genes that have general involvement in secondary metabolites production presents a challenge for further functional analysis. As 
these genes may play a part in several secondary metabolites production pathways, it is hardly possible to infer which one is currently activated based only on transcription data.

In C. parasitica there are two mating types, but three types of pheromones. Each strain can carry only one mating allele, mat-1 or mat-2, but it carries all three mating pheromones genes. Expression of pheromones depends on what mating allele is present in the haploid mycelium. Strains expressing MAT-2 activate $m f 2-1$ and $m f 2-2$ pheromone genes, but ones with MAT-1 activate the $m f 1-1$ pheromone gene. Strains used here for barrage experiment, P74-3 and EP155, are both MAT-2. Thus, MAT-1 pheromone $m f 1-1$ remains silent and we observe the expression of only MAT-2 related genes. C. parasitica reproductive genes are not part of UniProt/SwissProt reviewed database, so it was not possible to include them in the enrichment analysis with DAVID. However, these genes are easily detected with hierarchical clustering from Figure II-4. Genes $m f 2-1$ (ID: 85578), mf2-2 (NOV_010635) and mat-2 (ID: 44005) show the most dramatic change in expression of any genes during barrage. Here, mat-2 is a part of MAT locus, which can harbour one of two mating alleles (mat-1, mat-2). This gene encodes a transcription factor that activates downstream processes like pheromone production and fruit-body formation. Genes $m f 2-1$ and $m f 2-2$ encode precursors of sexual pheromones that depend on the MAT-2 locus (Marra and Milgroom 2001; L. Zhang et al. 1998).

We were not able to perform gene enrichment analysis with genes involved in sexual reproduction in $C$. parasitica. As a result, the "Sexual reproduction" group is mostly defined by yeast orthologs. These include the ascospore development Dit1-like protein (ID 220966, 265800) that plays a role in dityrosine synthesis at late stages of yeast ascospore wall maturation (Briza et al. 1994). It is expressed only in yeast 
sporulating cells and its inactivation makes spores more sensitive to high temperatures and lytic enzymes (Briza et al. 1990). On the regulatory side we find an ortholog of Ste11p (ID 351932), a transcription factor carrying HMG (High Mobility Group box) domain. This transcription factor activates sporulation and mating in baker's yeast (Kjaerulff et al. 1997). It is activated through external signals and it triggers mating through increased nuclear accumulation (Qin et al. 2003). In clustering analysis, 265800 and 351932 are found in Cluster 1 showing low differential expression. However, the second dit 1 homolog, 220966, is highly upregulated.

Also in the 'sexual reproduction' group is gene 51413 encoding an ortholog of transporter protein, Isp4, that is activated during sexual cycle in S. pombe (Lubkowitz et al. 1998). This gene is among the highly overexpressed genes in Cluster 8 (see Table S1). Activation of orthologs of genes functioning during sexual reproduction in S. cerevisiae and S. pombe may indicate interesting connections with filamentous fungi. Process like failed mating in yeast activates PCD in response to pheromones in absence of a suitable partner (N. N. Zhang et al. 2006b). Allorecognition in C. parasitica apparently activates similar processes, with exception that meiosis and sexual spore production are not set in motion.

Other DE genes in "Sexual reproduction" group include 262923 and NOV_004335 that encode homologs to tud protein found in fruit flies. The tud protein carries a Tudor domain and works as an essential part of nuage structure, activated during germline cell development. This structure employs PIWI, an RNAi complex of proteins that prevents activation of retrotransposons (Kibanov et al. 2011). Gene products of 262923 and NOV_004335 are the only two identified genes in the DE dataset that carry 
the Tudor domain; 262923 has the most homology to the fruit fly tud protein and NOV_004335 was identified among novel transcripts (see Table S1). NOV_004335 is homologous to an RNA helicase, which is involved in spermatogenesis and oogenesis where it inhibits transposons activation (Vagin et al. 2004). It is not clear how the transcription rate of these genes correlates with their RNAi function. In C. parasitica the TUD ortholog 262923 is overexpressed during barrage in all samples while NOV_004335 is found among downregulated genes in Cluster 2, showing insignificant change in expression among all samples except the Dicer knockout.

This brings us to a group of genes specifically attributed to "RNA interference". In this group, we find two homologs (IDs 74333, 261854) of Argonaute proteins from fruit flies and yeast. Both appear to be previously identified in C. parasitica as Argonaute-1 and Argonaute-4 genes (agll and agl4), respectively, but neither is included in the UniProt/SwissProt reviewed database. Previous research has not identified a particular specialization of these genes. Among four Argonauts and two Dicer genes in $C$. parasitica only the RISC complex formed by Dicer- 2 and Argonaute- 2 were found to be functioning as an antiviral defence (Sun et al. 2009a; X. Zhang and Nuss 2008). Others, Dicer-1 and Argonauts 1, 3, and 4, were not found to be involved in any known processes. According to our RNA-seq analysis both, agll and agl4 are moderately overexpressed and found in Cluster 1. Other genes involved in RNAi appear to not be differentially expressed during barrage. As the previous study indicated, expression of agl2 gene is important to activate expression of $d c l 2$ during antiviral defence (Sun et al. 2009a). It is possible that one or both of agll and agl4 activate dcll. Basically, the increase in Argonaute expression should be followed by an increase in Dicer expression. This allows 
us to suggest, that we observe the early stage of RNAi activation and that expression of $d c l 1$ will follow. In addition, previous studies reveal that RNAi proteins, Dicers and Argonauts, tend to specialise in types of RNA they silence (Siomi and Siomi 2009). There are Dicer-Argonaute complexes that deal exclusively with viral siRNA or ones producing miRNAs, which originate from a host genome or regulate transposon activity like a PIWI complex. At the same time, these genes demonstrate some level of redundancy when one of the Dicer genes or Argonaute genes is knocked out (Hutvagner and Simard 2008).

To summarise this section, we identified DE genes during barrage that are associated with RNAi and share significant homology to genes responsible for transposon silencing in germline cells in fruit flies. We identified two Argonauts, agll and agl4, activated during barrage, but, according to the literature, neither is known to be active against viral dsRNA. Following the logic of RNAi proteins "separation of labour" and in combination with other observations, we can suggest that agll and agl4 genes may play a role in silencing of transposable elements in the genome of $C$. parasitica. We can further suggest that activation of RNAi against transposons may be important during conidia formation. Studies of fungal transposons in Neurospora reveal that some may allow different species to overcome interspecies barriers, mate and produce fertile ascospores (Daboussi and Capy 2003). In that sense, transposon silencing may be seen as a part of an incompatibility mechanism. In the $C$. parasitica lifecycle, strains do not produce specialised male sexual spores and use conidia as male gametes (Turina et al. 2003). Association of RNAi transposon silencing during germline cells development is common among animals and insects (Girard et al. 2006; Houwing et al. 2007; Tam et al. 2008). 
From that perspective, it is reasonable to suggest that $C$. parasitica may employ mating pheromones and RNAi processes in conidia formation. If asexual conidia formation is considered as a part of the sexual cycle, the process of asexual conidia formation in $C$. parasitica must be treated as part of the germline development. Considering that it is likely that mating genes and RNAi are always activated during conidia development.

\section{INTERPRO/Pfam protein domains enrichment analysis}

A different perspective to the GO enrichment analysis of barraging strains is shown in Figure II-5b, using conserved INTERPRO/Pfam protein domains. Classifying proteins by conserved domains can provide additional insights into the function of genes that otherwise lack GO annotation. In protein domain enrichment analysis, the largest fraction of differentially regulated genes fall into the "Cytochrome p450" group. Most of the cytochromes here represent so called 'group I p450s'. It is the largest group of p450 monooxygenases that is involved in various processes, among which are detoxification and toxin biosynthesis (McLean et al. 2005; Uno et al. 2012). Some of these proteins were already mentioned as a part of 'toxin biosynthesis' GO terms. For example, the functionally similar genes $s t c F$, $s t c C$, stcS, which appear in up- and downregulated clusters. Also, aflatoxin biosynthesis involves the p450 domain factor aflL, a versicolorin B desaturase that catalyses a reaction of Versicolorin B into Versicolorin A (Yu et al. 2004).

Closer analysis of other UniProt IDs in this group in their relation to fungi revealed the most numerous group of paralogs (IDs: 339471, 261162, 345802, 346809), which are homologous to p450foxy from Fusarium oxysporum. p450foxy is an example of a self-sufficient p450 monooxygenase that is capable of functioning without the aid of 
an external p450 reductase (Kitazume et al. 2000). p450foxy and its analogs are believed to be responsible for denitrification performed by various fungi (Shoun and Takaya 2002).

The majority of other p450 genes upregulated during $C$. parasitica barrage are related to p450s involved in mycotoxins biosynthesis in other fungi. These include the ortholog (ID: 269746) of Fus8 from Fusarium fujikuroi, which is involved in fusarin biosynthesis (Niehaus et al. 2013). Also included is an ortholog of $\operatorname{ccs} G$ (ID: 274617) from Aspergillus clavatus that is part of the biosynthesis of cytochalasins E and K, two potent toxins and anti-angiogenic compounds (Qiao et al. 2011). Also upregulated is ID:353416, an ortholog of $\operatorname{ccs} C$, which is another gene from this pathway found in the alcohol dehydrogenase group.

In the downregulated fraction of DE p450 domain group, genes involved in synthesis of Oxilipins are of particular interest. Oxilipins have various functions, but most studied ones involve fungal virulence. In animal systems, one type of oxilipins produced by fungi, prostaglandins, can increase virulence of pathogenic Cryptococcus neoformans and Candida albicans by inhibiting the host immune system (Noverr et al. 2001). Interestingly, plant derived oxilipins inhibit the production of fungal toxins, like Aflatoxin and sterigmatocystin in Aspergillus spp. and thereby reduce fungal virulence (Burow et al. 1997). Within the fungus itself, oxilipins were demonstrated to function as hormones, regulating the balance of sexual and asexual spore production (Tsitsigiannis et al. 2005). It was shown that in $A$. nidulans decreased expression of ppoA (lipoxygenase, producing oxilipins) reduces sexual spore production (Tsitsigiannis et al. 2004). In case of C. parasitica, our analysis demonstrates that the ppoA ortholog (ID: 332509) expression was significantly decreased in barraging strains. Orthologs of 332509 (Pa ID: 
Pa_5_1240) in P. anserina were also downregulated during heterokaryon incompatibility (Bidard et al. 2013). This suggests that downregulation of lipoxygenase may be part of global inhibition of later stages of sexual cycle during vegetative incompatible interactions. In addition to control of sporulation, deactivation of ppoA in Aspergillus leads to increased resistance to ROS and higher virulence in animal models (Tsitsigiannis et al. 2005). These authors suggest that downregulation of $p p o A$ helps to resist host defence mechanisms. Similarly, this model may be applied to barraging cells in $C$. parasitica, as ROS and toxin production are both evident during barrage. Of interest, 332509 was not differentially expressed in incompatible interactions involving the Dicer2 knockout strain. This may indicate that 332509 requires RNAi to regulate its gene expression.

An interesting feature of the p450 and alcohol dehydrogenases (ADH) domain groups is that they combine both proteins involved in mycotoxins production and detoxification. Above we described genes with ADH domains in relation to Toxin Biosynthesis group of GO terms. Here we describe additional examples, among which are three homologs of fungal alcohol dehydrogenases. Two (IDs: 47533, 264580) encode homologs of ADH6 from S. cerevisiae and Zinc-type ADH from S. pombe. The third gene (ID: 106275) codes for an ortholog of adh-1 from N. crassa. Previous studies suggest that ADHs in fungi serve as detoxifying agents in dealing with alcohols, which are common products of sugar metabolism (LARROY et al. 2002). Consequently, ADHs were found to play a role as virulence factors in pathogenic fungi. As shown for $A$. fumigatus, ADH1 in hypoxic conditions helps to defend the fungus against rising levels of alcohol, where null-mutants show increased vulnerability to host defences (Grahl et al. 
2011). Expression of ADHs in C. parasitica during barrage varies among genes. Gene 264580 is upregulated only in P74-3+EP155 $\Delta$ dcl2 interactions, 106275 shows less upregulation in interactions involving p29 and dicer knockout strains compared to wildtype and EP155p29stop strains. Finally, 47533 is universally upregulated in all barraging samples. An increase in expression of ADH domain genes is expected considering that previous findings show that ADHs expression in fungi is partially regulated at the transcriptional level (Bertram et al. 1996). Presumably ADH domain genes are important components of detoxification mechanisms and are likely activated in non-barraging cells that are exposed to secreted toxins associated with barraging cells.

The group of MFS transporters domain superfamily finds its association with toxin effects as well. Many transporters work in conjunction with detoxification mechanisms. For example, when a protein like GST conjugates GSH to a toxic compound, the conjugated toxin must then be removed from cell (Tew and Townsend 2012). Then again, some toxic secondary metabolites must be excreted out of the fungal cell by a set of specialised pumps. In the MFS group, we identified two categories of transporters. One showing the closest homology to yeast uncharacterised transporters and a second type that are involved in toxin transport in fungi. In the MFS toxin transporter category, we identified 10 orthologs of known fungal mycotoxin transporters. Among them are four genes (IDs: $246669,355270,60905,340229$ ) that show significant homology to gliA from Aspergillus fumigatus and these present an interesting example of involvement of the fungal toxin system. gliA is a product of a very well studied gene cluster responsible for production of gliotoxin (Wang et al. 2014). One of the main functions of this transporter is to alleviate the effects of gliotoxin on the fungus itself (Owens et al. 2015). 
This activation of toxin export indicates that barrage may be similar to basic defence against invasive organisms.

Another interesting effect seen with enrichment analysis is how barrage affects some basic metabolic functions. Amino acid permeases are the group of transporter domains identified on Figure II-5 that are responsible for uptake of amino acids from the extracellular environment. These transmembrane proteins transport amino acids from the environment into the cell to be used as nitrogen source or in protein synthesis (Fischer et al. 1998). Amino acid permease transporters are transcriptionally regulated and activated by the presence of corresponding amino acids (Regenberg et al. 1999). All amino acid permeases in yeast are structurally similar, but demonstrate substrate specificity. Activation of gene expression depends on what amino acid is in demand by the cell (Regenberg et al. 1999). Among amino acid permeases in C. parasitica genome, we identified six yeast orthologs. All show little or no DE variance among samples and moderate upregulation in Cluster 1 during barrage. Activation of these genes during barrage is not very dramatic, but still may indicate a limitation on preferred nitrogen source and switch over to another. On the other hand, inhibition of substrate intake can be seen on another group of protein domains, sugar transporters. Here, we find gene orthologs of various permeases that are reported to transport compounds that are used as a carbon source. However, compared to the amino acid permeases group, carbon source transporters are more numerous in the downregulated fraction (Figure II-5b). Deactivation of carbon intake may in turn stimulate incompatible cells to activate PCD.

Overall protein domain analysis allowed us to identify pathways related to fungal virulence factors - including mycotoxins production and stress resistance mechanisms (in 
the form of $\mathrm{p} 450 \mathrm{~s}$, transmembrane transporters and $\mathrm{ADH}$ proteins). This allows us to conclude the most probable trigger of PCD in barraging cells are multiple toxins produced by both strains.

\section{Comparison to HI transcription profiles of $N$. crassa and $P$. anserina}

Transcriptional response to Heterokaryon Incompatibility (HI) was previously investigated in N. crassa and P. anserina (Bidard et al. 2013; Hutchison et al. 2009) and we can compare our results with these previous studies. However, first of all we need to elaborate on experimental differences among the three systems. Both of the previous studies employed a co-called induced incompatibility technique. Simply, this method uses a property of some $\mathrm{HI}$ factors to activate only at permissive temperatures. In the case of $N$. crassa, strains incompatible at the het-c locus can form heterokaryons in a temperature dependant manner if one of the strains carries mutant version of $p i n-c 2^{m}$ (Kaneko et al. 2006). This is type of HI in N. crassa also referred as TSinc (Temperature Sensitive incompatibility). In this example, incompatible strains het-cl/pin-cl can form a heterokaryon with het-c2/pin-c $2^{m}$ at $34^{\circ} \mathrm{C}$, showing very low death rate. However, when transferred to a lower temperature, $20^{\circ} \mathrm{C}, \mathrm{HI}$ is rapidly induced in these heterokaryons. In the $P$. anserina model, strains that are incompatible due to non-allelic interactions involving het- $R$ and het- $V$ present normal culture development at $32{ }^{\circ} \mathrm{C}$ whereas incompatibility reactions occur when these strains are transferred to $26^{\circ} \mathrm{C}$. These systems have the obvious advantage over the $C$. parasitica barrage model used in the present study. They allow for synchronous induction of incompatibility across the entire mycelium, providing a means to study the timing of incompatible events. In $C$. parasitica 
barraging experiment fusion time is desynchronised and some cells undergo self fusion. However, the induced incompatibility technique is limited to only temperature inducible HI systems and may not integrate processes such as hyphal contact and fusion that occur during barrage formation. As far as we know, C. parasitica does not have temperature inducible HI. Thus, we use a different technique to analyse incompatibility processes in barraging cells and this should be kept in mind when comparing our results to those from $N$. crassa and $P$. anserina. We are also comparing incompatibility reactions triggered by non-orthologous incompatibility genes in the three species and processes may vary accordingly between these systems.

A final difference to consider is that the two previous transcriptome studies on induced incompatibility profiling in $N$. crassa and $P$. anserina were performed using microarray technology (Bidard et al. 2013; Hutchison et al. 2009). For our study, we used Illumina Next Generation Sequencing to gather transcriptome data. Illumina NGS has some advantages over use of microarrays. Specifically, a specialized chip is not required and any region of the genome that is transcribed can be analysed by NGS technology. Nevertheless, direct comparisons are possible as all three methods essentially estimate relative gene expression on the $\log 2$-fold scale.

With the above differences in mind, we compared close gene orthologs (BLAST+ score over 350) of $N$. crassa and P. anserina from the C. parasitica genome (Figure II-6 and 7). Out of 13944 C. parasitica genes, over 60\% (BLAST threshold e-value $<10^{-10}$ ) show homology to 


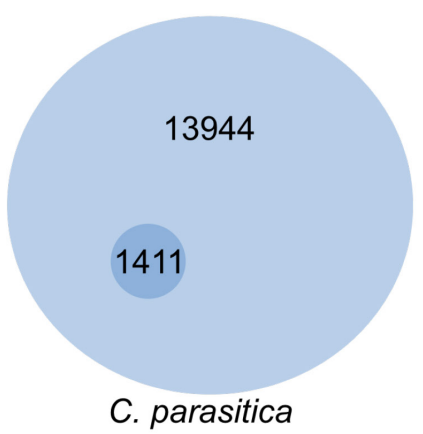

B

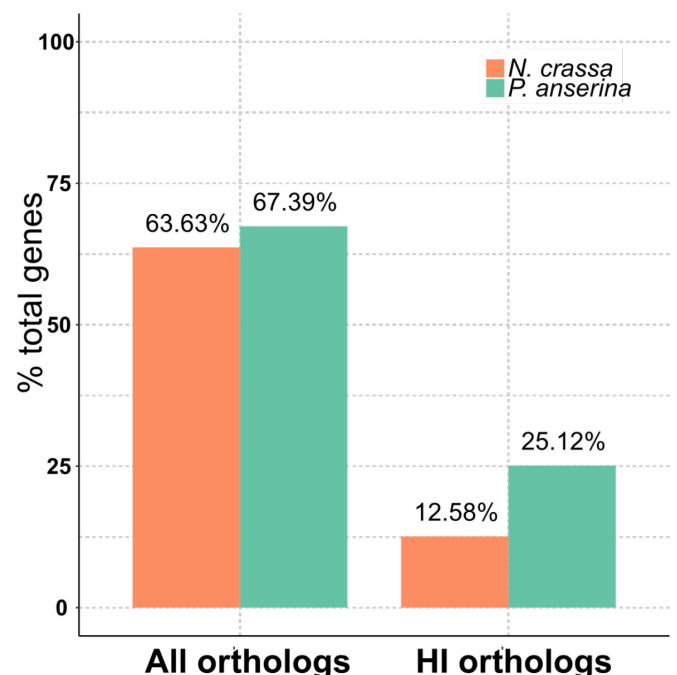

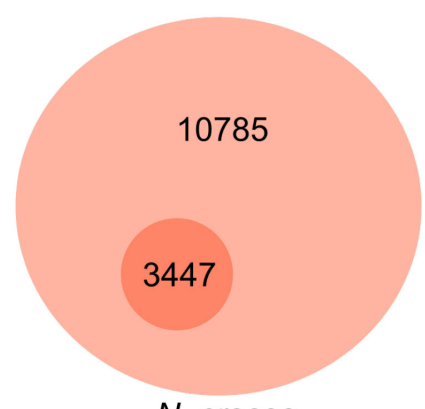

N. crassa

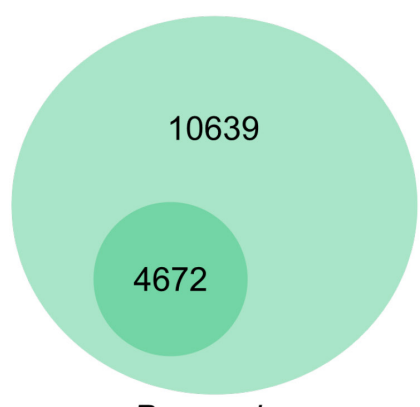

P. anserina

C

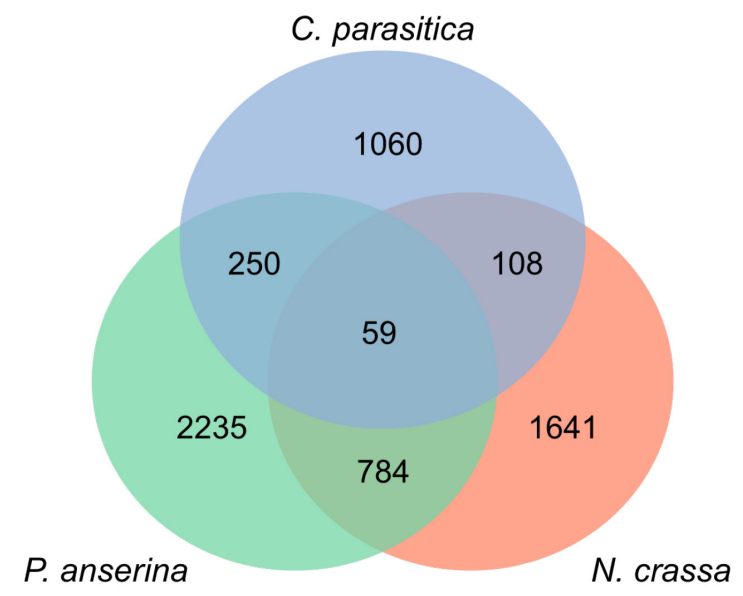

Figure II - 6. Orthologs of $N$. crassa and $P$. anserina identified in $C$. parasitica genome. A) Diagram of relative proportion of differentially expressed genes HI (N. crassa or $P$. anserina) and barrage formation ( $C$. parasitica, this study). Large circles represent total number of genes in genomes of three species. Size of the small circles inside shows proportional share of differentially expressed genes during HI or barrage. B) "All orthologs" represents the proportion of all $C$. parasitica genes that have identified orthologs in $N$. crassa or P. anserina (e-value $<10^{-10}$ ). "HI orthologs" represents the proportion of orthologs that are differentially regulated during heterokaryon incompatibility ( $N$. crassa or $P$. anserina), over all genes in $C$. parasitica. C) Venn diagram of "HI orthologs" differentially expressed during HI in P. anserina, N. crassa and C. parasitica. 
both $N$. crassa and P. anserina. We used the reference genome from $N$. crassa OR74a that includes 10785 protein coding genes, from which 3447 (32\% of total genes in genome) were significantly differentially expressed during het-c-associated HI (Figure II6a) (Hutchison et al. 2009). In the $C$. parasitica dataset we defined differentially expressed genes as significant when the DE value was above two $(\log 2$ fold $>2)$. In contrast, DE values from the $N$. crassa study were below this value and we came to use only 2000 (18\% of total genes in genome) out of 3447 HI DE genes. Finally, when we select $N$. crassa DE genes, we need to identify how many of them show homology to $C$. parasitica genes overall, and $\mathrm{DE}$ genes during vic3-incompatibility in particular. As shown on Figure II-6a we identified 6863 or $63 \%$ of $C$. parasitica genes that show significant (BLAST threshold e-value $<10^{-10}$ ) homology to $N$. crassa genes. Out of the 2000 N. crassa HI DE genes, only 1357 (67\% of DE HI genes) show homology to $C$. parasitica genes. Similarly, for the $P$. anserina comparison we used the genome from the S mat + strain that contains 10639 protein coding genes. Out of these genes, 4672 (44\% of total) were identified as significantly (DE threshold $>2$, p-value $<0.001$ ) differentially expressed as a result of $\mathrm{HI}$ (Bidard et al. 2013). Among total P. anserina genes 7170 (67\% of total) showed significant homology to C. parasitica. Among DE affected by HI in $P$. anserina, 2672 (57\% of DE HI genes) showed significant homology to C. parasitica (Figure II-6b). In comparison to the other two species, C. parasitica vic3 incompatibility causes differential expression of 1411 (10\% of total) genes. As we can see, induced HI in P. anserina and $N$. crassa causes a much larger change in gene expression profiles than we observe in barraging C. parasitica. This may suggest that induced $\mathrm{HI}$ in $N$. crassa and $P$. anserina results in a larger impact on fungal cells compared to strains undergoing 
barrage or that differences in methodologies (discussed above) obscures some DE genes in C. parasitica.

Out of all $\mathrm{HI}$ orthologs, only 59 genes are DE during incompatibility reactions in all three species (Figure II-6b). Pairwise correlation analyses among DE HI genes between C. parasitica and N. crassa $(\mathrm{p}$-value $=0.1)$ or $C$. parasitica and P. anserina $(\mathrm{p}$ value $=0.3)$ was not statistically significant. However, there is a strong correlation between $N$. crassa and $P$. anserina $\mathrm{HI}$ genes differentially expressed during $\mathrm{HI}$ (calculated $\mathrm{p}$-value $<10^{-6}$ in this study and p-value $<10^{-4}$ by Bidard et al. (2013)). This, again, implies possibilities that there are functional differences between induced $\mathrm{HI}$ and naturally occurring barrage, or that the three systems may exhibit species-specific or incompatibility locus-specific differences.

Even considering these differences we still can point out several similarities between C. parasitica and the other two HI responses at the transcript profile level. As seen on Figure II-6c there are 59 orthologs that are differentially expressed in all three species during incompatibility reactions (see Table S3). A majority of these genes are downregulated in P. anserina and $N$. crassa. This narrows our analysis as only 8 out of the 59 genes were downregulated in all three species. As for upregulated genes for all three species, these are mostly eliminated by $N$. crassa data, as only two of its genes are upregulated above threshold. Interestingly, both are also upregulated in P. anserina and $C$. parasitica. One (ID: 336241) encodes uncharacterised ATP dependant calcium transporter, similar to Pmcl (UniProt ID: Q9HDW7) from yeast. The second (ID: 264062) is similar to yeast hydrolase (UniProt ID: O14158). Both proteins are poorly characterised and do not allow us to infer practical conclusions. 
Among upregulated genes, one functionally similar group worth noting is detoxification genes. As a particular example, the ortholog of Quinone Oxidoreductase (QO, ID: 333952) is part of the antioxidant response system in animal (Johnson et al. 2008). 333952 is highly overexpressed in C. parasitica barrage, but downregulated in $N$. crassa and P. anserina. Along with QO, two Glutathione-S-transferase (GST) genes (IDs: 357090, 58765) are also found among differentially expressed genes in all three species, with only single homologs represented in $P$. anserina (ID: Pa_1_5100) and N. crassa (ID: NCU05780). GSTs present most highly upregulated genes in $P$. anserina and $C$. parasitica, but in N. crassa GST showed slight downregulation. GSTs and QO are transcriptionally regulated by Nrf2 transcription factor which increases QO and GST expression in response to ROS, stress and toxins exposure (Jaiswal 2000; Tew and Townsend 2012). GST is responsible for neutralization of toxic compounds by binding Glutathione (GSH) to them to make them more accessible for transport out of the cell. Balance of GHS in the cell is very important for survival and when it drops due to high toxic exposure, it may lead to apoptotic death (Circu and Aw 2012). These detoxification proteins play an antiapoptotic function neutralising toxic compounds. Considering this, it is no surprise to see QO and GST overexpressed during barrage. However, the downregulation of GST and QO during induced HI may indicate that C. parasitica barraging hyphae may also downregulate detoxification to speed up onset of PCD. That is, increased detoxification signals in C. parasitica data may come from cells exposed to toxic environment produced by barraging hyphae.

Genes universally downregulated in all three species present a particularly interesting example of an ortholog of the Arabidopsis neutral Ceramidase (see Table S3; 
IDs: 100328, NCU04721, Pa_4_6950). This protein catalyses degradation of Ceramide to Sphingosine. Various studies on tumor cells in animals showed Ceramide promotes apoptotic signals coming from TNF receptors (Obeid et al. 1993). Sphingosine, on the other hand was shown to inhibit apoptosis and promote cell growth in animals (Ohta et al. 1994). Also, a previous study indicated that activity of ceramidase in combination with growth factors promotes growth of human fibroblast cell culture (Coroneos et al. 1995). Additionally, it was shown that induction of Ceramide can downregulate cytochrome p450 2C11 in mice, a process that mimics Interleukin-1 signaling (Nikolova-Karakashian et al. 1997). Downregulation of cytochrome $\mathrm{p} 450 \mathrm{~s}$ by Interleukin-1 activation is proposed to be part of the immune response making rat cells more prone to death by decreasing defence mechanisms (J. Chen et al. 1995). As we showed previously in this chapter, proteins with p450 domains are the most numerous group of DE genes and are mostly upregulated during barrage (see Figure II-5). Thus, we can suggest that accumulation of ceramide in cells, due to ceramidase inhibition, can downregulate only a small fraction of cytochrome p450s. Thus, we can infer that downregulation of ceramidase during HI may inhibit growth processes and favor apoptosis-like response.

All factors mentioned in the above section are indirectly involved in PCD. Here we will examine differential expression of factors associated with well-studied examples of PCD. A model for PCD in fungi was first described for yeast, where multiple orthologs of animal apoptotic genes were identified (Carmona-Gutierrez et al. 2010). However, from the very beginning, fungi demonstrated distinctive PCD characteristics. Many crucial regulators of apoptosis in mammals, such as BCL2 proteins, do not have direct orthologs in yeast. Studies showed that expression of human BCL2 proteins in 
yeast can, nevertheless, promote or suppress PCD (Sato et al. 1994; Q. Xu and Reed 1998). Similarly, cysteine proteases that demonstrate caspase-like activity in plants and fungi do not share significant homology to mammalian versions and for that reason were termed metacaspases (Tsiatsiani et al. 2011). So far in yeast only the intrinsic type of apoptotic death has been identified. It was associated with activation of YCA1 metacaspase and release of Cytochrome c, AIF and EngoG due to mitochondrial membrane permeabilization (Carmona-Gutierrez et al. 2010). Disruption of these genes leads to resistance to apoptotic death (Guaragnella et al. 2006; Khan et al. 2005). Activation of PCD in yeast usually happens as a result of exposure to toxic conditions like ROS, acetic acid or sometimes to opposite sex pheromones (F. Madeo et al. 2002; Pozniakovsky et al. 2005; Severin and Hyman 2002; Wissing et al. 2004; N. N. Zhang et al. 2006b).

However, cell death mechanisms associated with HI seem to show distinctive nature from these known yeast PCD processes. Previous analysis showed that orthologs of 11 yeast apoptosis genes are not activated during HI-associated PCD in N. crassa (Hutchison et al. 2009). Disruption of several of those genes in yeast leads to resistance to apoptotic death (Guaragnella et al. 2006; Khan et al. 2005), but in the case of $N$. crassa disruption of two metacaspases (NCU09882, NCU02400 ${ }^{1}$ ) and AIF (apoptosis-inducing factor, NCU05850) does not impede HI-associated PCD (Hutchison et al. 2009). Eight of these $11 \mathrm{~N}$. crassa genes have identifiable orthologs in the $C$. parasitica genome, but their expression was not influenced by the barrage process (data not shown). These genes include orthologs of two metacaspases (NCU09882, NCU02400), Cytochrome c

\footnotetext{
${ }^{1}$ Neurospora crassa genome portal (protein name field): http://genome.jgi.doe.gov/Neucr2/Neucr2.home.html
} 
(NCU01808), Ste4p (Cp ID: 105373, Nc ID: NCU00440), G-protein beta-subunit (NCU00440), Lag1 (NCU00008), Ppa1p (NCU09747), HSP70 (NCU09602) (Hutchison et al. 2009). Other apoptotic genes identified in yeast similarly do not show differential expression during barrage in C. parasitica. Most of these genes are related to protein kinases cascade and G-protein function and are discussed in detail in Chapter IV.

A common property in $C$. parasitica barrage and $N$. crassa induced $\mathrm{HI}$ is production of ROS. In N. crassa, genes involved in ROS response, such as Cytochrome c, NADPH oxidase and glutaredoxin are overexpressed during HI (Hutchison et al. 2009). In $C$. parasitica, on the other hand none of these genes are differentially expressed in barraging strains. However, this does not indicate lack of ROS accumulation in $C$. parasitica during barrage formation. On the contrary, in some of the work described latter in this thesis (see Chapter IV) indicates that antioxidant proteins like GST, QO, thioredoxin reductase, glycosyl transferase and AP enduclease 1 (APE) show an increased expression during barrage. Staining with dichlorofluorescein (DCF), a ROS indicator, allowed us to confirm ROS production during barrage formation, similar to what is seen in N. crassa (Hutchison et al. 2009). As far as we know, no similar experiments were performed for $P$. anserina $\mathrm{HI}$ and transcriptome analyses have not directly identified activation of genes related to ROS production similar to what is seen in N. crassa and P. anserina (Bidard et al. 2013). However, protein domains related to GST, QO, Cytochrome p450 proteins were found to be abundantly upregulated in P. anserina HI strains. This data suggests that production of ROS and associated programmed cell death mechanisms may present a common feature among these filamentous fungi. 
Lastly, upregulation of HET domain genes was identified in the transcriptome data sets for all three species. C. parasitica and N. crassa showed very similar proportions of activated HET genes (Hutchison et al. 2009). There were only five HET domain genes found as differentially expressed in N. crassa dataset, but surprisingly, four of them were downregulated. The one upregulated HET gene is a hypothetical protein (NCU03507) showing similarity to het-6 (NCU03533) and appears upregulated one hour after HI induction (temperature decrease). Among the other four downregulated genes, we see the het- $6^{\mathrm{OR}}$ (NCU03533) allele and another uncharacterised HET domain gene (NCU09045), with the remaining two HET genes identified as het-cl (NCU03125) and het-c2 (NCU07947), both are alternate alleles from the het-c locus that activated incompatibility in the study (Hutchison et al. 2009; Kaneko et al. 2006). All downregulated HET genes appear late, after $8^{\text {th }}$ hour from HI induction. Similarly, $C$. parasitica showed only seven differentially expressed HET domain genes in barraging strains out of 124 predicted HET domain genes in the genome. In contrast, the $P$. anserina data set showed that out of total 130 predicted $P$. anserina genes in entire genome more than $50 \%$ are activated during induced HI. Even though C. parasitica and $N$. crassa have a smaller number of upregulated HET genes compared to P. anserina, all three species are able to demonstrate a similar spectrum of incompatibility reactions. As described previously, HET domain genes are sole activators of incompatibility reaction, and activation of single HET gene is enough to produce the entire spectrum of incompatibility symptoms (Paoletti and Clavé 2007). Thus, in our analysis we can additionally point out that the number of HET genes activated during barrage or HI may not influence the severity of incompatibility reaction. Also, we were able to confirm that 
no matter what type of incompatibility reaction is taking place, all involve transcriptional activation of HET domain genes.

As a final remark, analysis of 59 overlapping genes shows that $N$. crassa and $P$. anserina, at least in part, demonstrate activation of toxin biosynthesis genes, similar to $C$. parasitica (see Table S3). In the table we can identify at least three mycotoxin genes: two Trichodiene oxygenases (p450; ncID: NCU09103, NCU09103; paID: Pa_3_2900, Pa_1_23520, cpID: 251671, 262538) and Aflatoxin aldehyde reductase (ncID: NCU07240, paID: Pa_2_14080, cpID:357339) orthologs. Interestingly Aflatoxin aldehyde reductase is universally downregulated in all three species. To summarise our observations on similarities for barrage in C. parasitica and induced HI in N. crassa and $P$. anserina, all are associated with differential expression of various HET domain genes, increased ROS production and genes related to mycotoxins biosynthesis. 
Figure II - 7. (next page) Gene differential expression (DE) shown on C. parasitica genome map (11 largest scaffolds).

Diagrams from outside to inside: Black lines show genome map of 11 scaffolds, each tick mark corresponds to $100,000 \mathrm{bp}$. Inside the outer black lines, blue marks indicate previously annotated genes on JGI $C$. parasitica genome portal and orange marks indicate novel genes. Novel genes are transcripts which demonstrated detectable level of expression in areas of genome previously not annotated on genome portal. First circle shows expressed genes, and second circle shows DE genes during barrage. Interior to this are $C$. parasitica gene IDs of DE genes where black IDs indicate genes differentially expressed during barrage only in $C$. parasitica, green and purple IDs indicate that orthologs of those genes are DE during Heterokaryon Incompatibility (HI) in N. crassa and $P$. anserina respectively. The inner-most pink, grey and blue bands show plots of individual genes expression. The $\mathrm{Y}$ axis indicates genes differential expression in $\log 2$ scale. Y axis pink colour indicates upregulated $(y>2)$, blue - downregulated $(y<-2)$ and grey $(2>y>-2)$ indicate non-DE genes. Red dots indicate $C$. parasitica genes, green and purple dots indicate DE orthologs during $\mathrm{HI}$ in $N$. crassa and P. anserina respectively. 


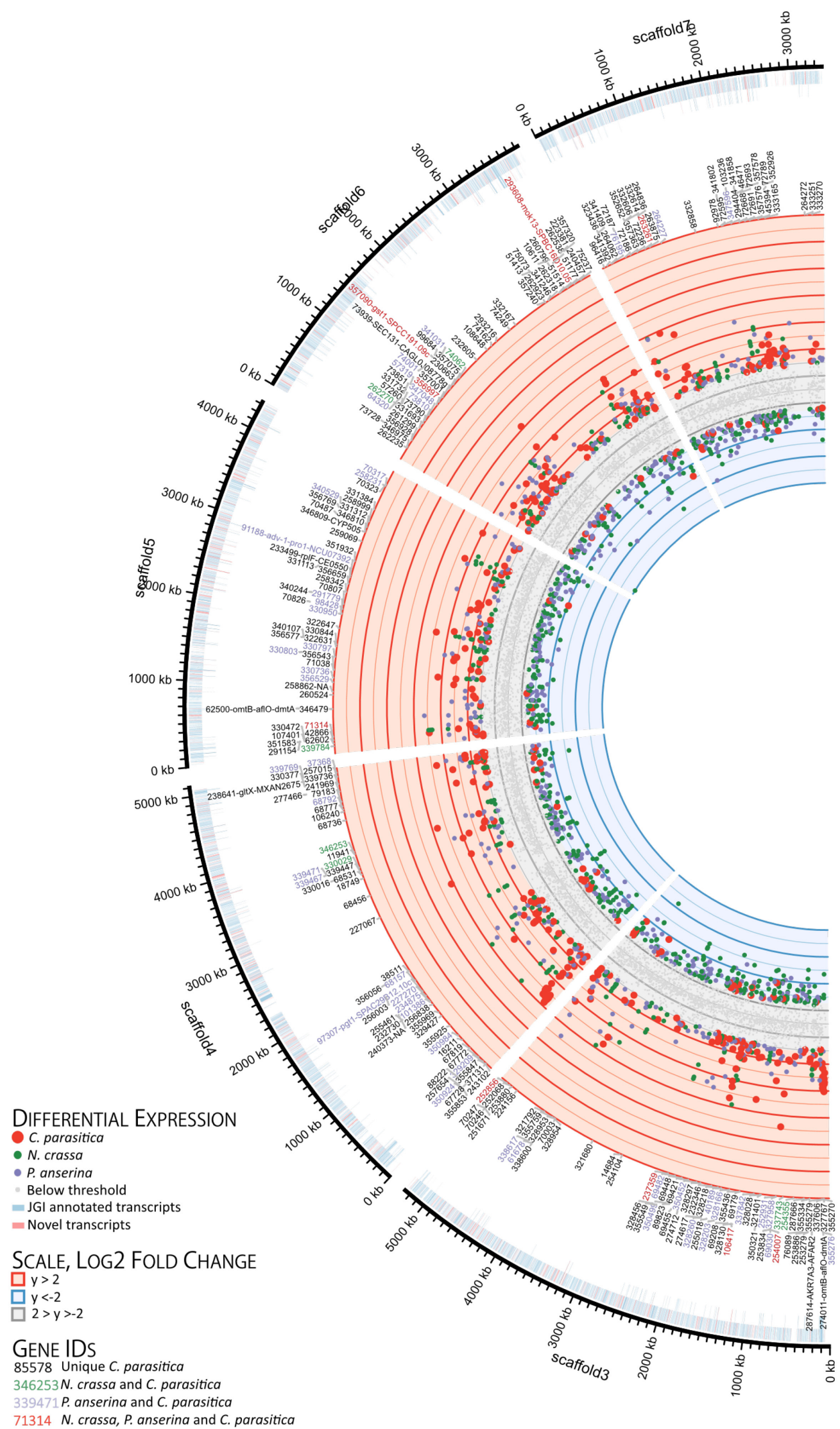




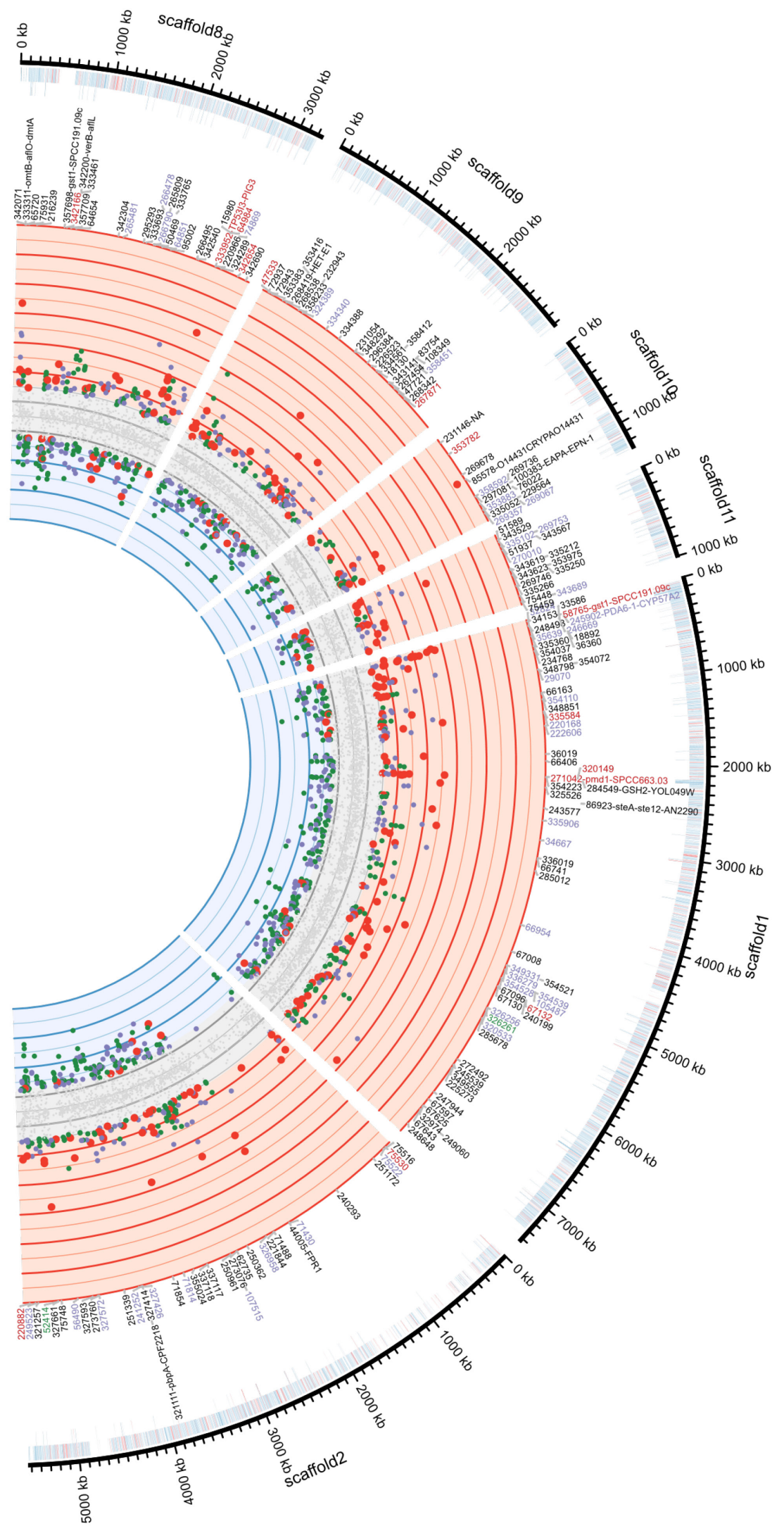




\section{Conclusion}

In this chapter we presented the results of RNA-seq analysis of vic3-associated incompatibility. We identified 1411 differentially expressed genes in barraging samples. Hierarchical clustering and gene enrichment analyses allowed us to functionally classify DE genes (see Figure II-4,5) and to identify processes related to fungal virulence and stress response. Activation of genes related to toxin biosynthesis indicates an active defence reaction against an invading organism and may resemble typical defence response found in other organisms, including plants. At the same time increase in expression by genes related to detoxification indicates that toxic environment created by barrage may trigger a stress defence mechanism in non-barraging hyphae.

Finally, comparisons to previous studies on $N$. crassa and $P$. anserina demonstrated critical differences and similarities between induced heterokaryon incompatibility (HI) in these other species and barrage in C. parasitica (Figure II-7). To compare 3 organisms together we used orthologs of $N$. crassa and P. anserina genes identified in C. parasitica genome (see Figure II-6).

Previous studies found that HET-domain genes are the main determinants of incompatibility reactions in Ascomycota (Glass and Dementhon 2006; Smith et al. 2000). Here we identified that both types of incompatibility reactions, barrage and HI, show increase in HET-domain genes expression. HI in $P$. anserina showed the most dramatic activation of HET genes compared to the other two species. More than $50 \%$ of HET domains were differentially expressed during $P$. anserina $\mathrm{HI}$ with $40 \%$ upregulated and 10\% downregulated (Bidard et al. 2013). It is surprising how robust HET genes activation 
is in P. anserina, considering that in the experiment genetic polymorphism was defined only by the het-R/het-V loci. In $N$. crassa HI showed only five differentially expressed HET genes, among which only one is upregulated (Hutchison et al. 2009). It is particularly interesting that the het-c2 gene was downregulated, considering the incompatibility setup in that study was based on het-c/pin-c polymorphism. Peculiar features of $N$. crassa HET genes activation appears to be time dependant. Upregulation of HET genes detected within 1 hour after HI was triggered, but downregulated HET genes appear only at the $8^{\text {th }}$ hour of HI. Here, it is possible to suggest that similarly to $P$. anserina HET activated at the beginning of HI, but then transcript levels drop due to degradation. In $C$. parasitica we found seven HET genes were upregulated during barrage. In addition, genes from vic3 locus which defines incompatibility in our experiment, but do not contain HET domains, were also upregulated (see Table S1). Similarly to the other two species, the majority of the upregulated HET-domain genes are not associated with any known barrage reaction in $C$. parasitica, with the exception of one HET gene, vicla, which as the name implies, functions as an incompatibility factor at the vicl locus. This indicates that, similarly to P. anserina, C. parasitica HET genes show an interesting functional redundancy during incompatibility. Genes associated with vic3 do not have HET domain but, nevertheless, activate other HET genes. In this sense, an incompatibility locus, vic, can be generally defined as a trigger capable of activating one or more HET-domain genes. In turn, HET genes likely trigger various toxin gene clusters, which cause a failure of anti-apoptotic defence mechanisms and activation of PCD. 


\section{CHAPTER III. Effects of CHV1-EP713 p29 protein and Dicer-2 knockout on barrage formation}

\section{Introduction}

One of the proposed functions of allorecognition is to restrict transmission of cytoplasmic genetic elements, like viruses, from infected to uninfected individuals (Caten 1972; Cortesi et al. 2001; van Diepeningen et al. 1997). Using the C. parasitica model, it has been demonstrated that PCD associated with allorecognition negatively correlates with Cryphonectria hypovirus (CHV1) transmission (Biella et al. 2002); the more rapidly vicassociated PCD occurs, the lower is the frequency of virus transmission. Also, it was shown that CHV1 p29 protein is able to reduce the death rate in vic3-incompatible strains (Tanha 2008). Similarly, a recent study showed that mycovirus of Sclerotinia sclerotiorum is able to downregulate het and other genes to inhibit barrage formation and allow for virus transmission (S. Wu et al. 2017). These observations make sense from an evolutionary perspective: allorecognition creates pressure on mycoviruses, forcing the viruses to develop mechanisms in order to circumvent the barrier. From data presented so far in this thesis it is possible to see that the effects of CHV1-derived p29 on fungal molecular pathways are pleiotropic and it is thus difficult to pinpoint particular PCD mechanisms affected (T. D. Allen and Nuss 2004; Segers et al. 2004). In light of the above, a more detailed dissection of molecular mechanisms involving allorecognition associated PCD and CHV1 infection is needed.

CHV1 is of particular interest as a potential biological control agent to reduce the virulence of $C$. parasitica. When a $C$. parasitica strain is infected with CHV1, it 
demonstrates a reduced virulence phenotype, termed hypovirulence. CHV1 inhibits pigmentation, sexual reproduction, and reduces conidiation rates in infected strains (Hillman and Suzuki 2004). The most damaging effects of C. parasitica are inflicted upon trees from the genus Castanea, the most valuable species of which are Castanea C. sativa in Europe and C. dentata in North America (Brewer 1995; Milgroom and Cortesi 2004). When a chestnut tree is infected with a virulent $C$. parasitica strain, it dies before it can reach a reproductive stage. When a hypovirulent $C$. parasitica strain infects a chestnut tree, it does not cause lethal damage, which in turn allows the host tree to survive and reproduce. There have been several studies investigating how CHV1 affects molecular processes in C. parasitica to produce hypovirulence (Hillman and Suzuki 2004). Earlier studies concentrated on activities of specific proteins or on the altered expression of certain genes such as lacl (coding for Laccase-1) in virus-infected and uninfected strains (Kazmierczak et al. 1996). More recent studies utilise Expressed Sequence Tags (ESTs) and microarrays to examine whole transcriptional profiles of infected strains. These latter studies confirmed pleiotropic effects of CHV1 on $C$. parasitica, demonstrating changes in expression with respect to hundreds of genes (Todd D. Allen et al. 2003; T. D. Allen and Nuss 2004). In our current work, we attempt to use NGS of RNA to further evaluate the effects of CHV1-p29 on C. parasitica gene expression. Following this we explore effects of CHV1-p29 protein on cell death rate during barrage formation associated with vic3-incomaptibility. Also, using gene expression data from monocultures we compare effects of p29 on C. parasitica gene expression with analyses performed in previous studies (Todd D. Allen et al. 2003; T. D. Allen and Nuss 2004). 
The bulk of previous research on CHV1 biology and genetics identified genes most vital for mycovirus reproduction (Suzuki et al. 1999; Suzuki et al. 2003). These identified a single protein encoded in the CHV1 genome, the papain-like protease, $\mathrm{p} 29$, which determined all CHV1 major phenotypic effects encompassed as hypovirulence (see Figure I-9, Chapter I). This data allowed us to simplify our model and avoid using whole CHV1 genome and instead make C. parasitica strains which express the p29 protein. One strain we used, EP155p29, had a fully functional p29 gene from viral strain CHV1EP713, and a second strain, EP155p29stop, contains a p29 gene with three stop codons inserted into the sequence so that it is not translated into protein. Previous work using Western blot showed the presence of p29 protein in EP155p29 strains, but in EP155p29stop strains this protein was never detected (Tanha 2008). One of the most vital functions of p29 in CHV1 biology is the inhibition of fungal RNA interference (RNAi) responsible for antiviral defence. Studies have shown that only Dicer-2 and Argonaute 2 ( $d c l 2$ and $a g l 2)$ are needed for antiviral defence and only that part of RNAi is influenced by CHV1 (Segers et al. 2006; Segers et al. 2007). As was mentioned in Chapter II, other RNAi protein, Dicer-1 and three Argonautes, are not triggered by presence of dsRNA.

In this chapter I analyse the effects of p29 protein on vic3-associated barrage. In order to isolate effects of Dicer-2 inhibition from effects of p29 on PCD we also examined barrages with a strain with the Dicer-2 gene knocked out (EP155 $\Delta$ dc12). The Dicer-2 knockout and genomic expression of p29 guarantee uniform effects on every cell in the strain. 


\section{Material and methods}

Strains and growth conditions. Strains incompatible by the vic3 locus, P74-3 and EP155-derived strains were used. EP155-derived strains include the wildtype strain EP155wt, the EP155p29 strain expressing p29 RNA and protein, the EP155p29stop strain expressing p29 RNA only, and the strain EP155 dcl2 that has the Dicer-2 gene deleted. For barrage tests and RNA extractions we used protocols outlined in detail in Chapter II.

Microscopy assay. Programmed cell death rate during vic3 barrage was assessed using the microscopy protocol described in Biella et al. (2002). Strains were inoculated on microscope slides coated with PDA. Inoculum block of $\sim 0.5 \mathrm{~mm}^{3}$ were taken from the edges of 7-10 days old cultures grown on PDA. These agar cubes with mycelium were placed on microscopy slides approximately $1 \mathrm{~cm}$ apart. Slides were incubated in a damp chamber for 2 days in dark at room temperature. After two days, inoculum agar cubes were removed and a staining solution was applied composed of $10 \mu$ Evans Blue dye ( $0.1 \%$ solution in water) mixed with $90 \mu 1$ of PDB medium.

RNA preparation. Total RNA was extracted on $3^{\text {rd }}$ day of growth. Concentration was adjusted to $1500 \mathrm{ng}$ per sample. Sequencing was performed on Illumina NextSeq platform.

RNA-seq data preparation. The quality of raw sequencing data was analysed with FastQC. Trimmed and filtered reads were aligned to $C$. parasitica reference genome using TopHat2 (Kim et al. 2013). Transcripts detection was accomplished with Cufflinks v2 (Pollier et al. 2013; Trapnell et al. 2012). Gene differential expression analysis was 
estimated with R package DESeq2 (Love et al. 2014). For a detailed description of materials and methods used in this analysis, see corresponding sections in Chapter II.

\section{Results and Discussion}

In Chapter II we discussed gene differential expression (DE) only in the context of pairings undergoing barrage formation of EP155-derived strains with incompatible strain P74-3. In this chapter, I assess the effects of p29 or Dicer-2 deletion on barrage formation. Additionally, we are now able to look at how p29 affects gene expression changes in strains growing in monocultures. Likewise, taking EP155wt (wild-type) as a control, we can test the other three monocultured EP155-derived strains, EP155p29, EP155p29stop and EP155 $\Delta d c l 2$, to analyse p29 and Dicer-2 deletion effects. This in turn will allow us to compare our data with previous research on transcriptional effects of CHV1 infection. However, our main interest with this study was to identify possible effects of p29 on barrage formation to infer possible mechanisms on how p29 has the capacity to influence vic3-associated barrage formation.

Using fluorescent microscopy, we were able to estimate the death rate in fused hyphae of four pairs of vic3-incompatible strain combinations (Figure III-1). Notable in the figure is that confrontations between P74-3 and EP155p29 and between P74-3 and EP155 dcl2, have a significant reduction in the overall death rates. It is important to note that these differences can be observed only in early stages of barrage formation (data collected on second day after pairing), because latter stages of barrage have similar death 

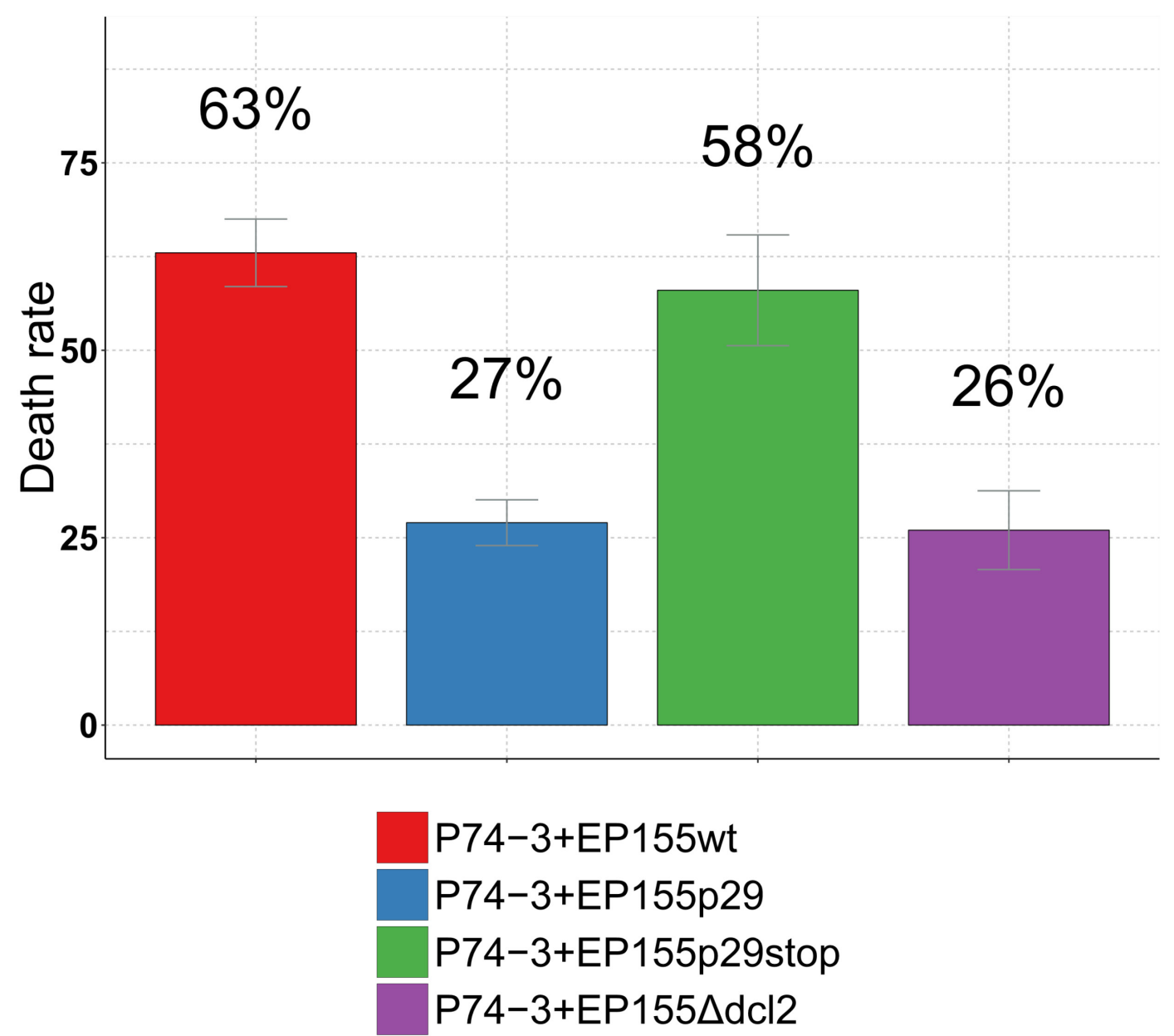

Figure III - 1. Cell death rate estimations using fluorescent microscopy.

Individual strains, grown on PDA plates for approximately eight weeks were paired on microscope slide and grown for two days. Slides were stained with vital dye Evan's Blue and observed under fluorescent light. Death rate was calculated as ratio of dead cells in identified contacts between incompatible strains and total number of identified contacts between two strains. Bars show mean death rate values (hyphal contacts exhibiting $\mathrm{PCD} /$ total hyphal contacts observed). The whiskers indicate standard error based on at least 50 contacts in each of 5 replicate experiments. 
rates (data not shown). As indicated in a previous study, reduced death rates during barrage correlate with increased virus transmission (Biella et al. 2002). This allows us to conclude that p29 can reduce death rate during barrage and possibly increase CHV transmission rates.

Previous wide-ranging transcriptomic studies on how $\mathrm{CHV} 1$ affect gene expression in C. parasitica were conducted using EST-based techniques. In these studies, custom microarrays with about 2200 C. parasitica genes were used to assess expression (Dawe et al. 2003). In comparison, modern sequencing methods, like Illumina NGS used in our current study, allowed us to detect more than 13,000 transcriptionally active sites ( $\sim 98 \%$ of genes in genome, see Figure II-2). Furthermore, the functional annotation of genes in EST analysis was performed by using BLAST+ search through NCBI protein database. Needless to say, the NCBI database has grown significantly over the last 15 years, whereby other databases like UniProt used in the current study did not even exist 15 years ago. While major databases have grown in size, annotations for filamentous fungi remain largely underrepresented. The most thorough functional genome and transcriptome annotations are still limited. For this reason, our current work is focused on choosing wider range functional annotation sources to account for the lack of deep specific functional annotations for $C$. parasitica genome and transcriptome (see Chapter II for further details on annotation and Figure II-2).

Previous analyses made with EST microarrays targeted the effects of CHV1 on gene expression (Todd D. Allen et al. 2003). These studies found that out of 2200 genes, CHV1 affects expression of 295 (13\%) genes. Following this, several authors compared effects of mild and severe types of CHV1 (T. D. Allen and Nuss 2004). Here, the severe 
form of CHV1-EP713 affected 295 genes, while the mild form of CHV1-Euro7 changed the expression of only 166 genes. The expressed p29 in EP155 genome that we used is from the severe CHV1-EP713 virus. Phenotypic changes caused by the expression of p29 corresponded to major symptoms caused by the entire CHV1 genome infection; specifically we observed reduced pigmentation, conidiation and faster growth rate in EP155p29. Thus, we expected similar reactions on a molecular level. Although we used p29 from severe CHV1 type, the number of affected genes in our RNA-seq data corresponded more with the mild CHV1-Euro7 form. In RNA-seq analysis, we identified only 139 genes $(\sim 1 \%$ of total genes) differentially expressed in EP155p29 strain monoculture compared to EP155wt. Considering that in this work the number of total genes was six times larger, the amount of genes influenced by p29 did not correspondingly grow compared to previous works.

To see the influence of p29 on barrage formation we can use gene expression hierarchical clustering described earlier in Chapter II. Figure II-4 shows genes differentially expressed during barrage clustered based on their DE value. For this analysis, we will use data from Figure II-4 panel B. The panel demonstrates collapsed clusters from panel A, in which DE values were averaged for each strain. Using estimated sample DE variance for each cluster as a guide, I was able to infer how certain genes are affected in individual sample conditions. In this case, those conditions are represented by the presence of $\mathrm{p} 29$, $\mathrm{p} 29$ stop or $\Delta d c l 2$ compared to control (averaged EP155 + P74-3).

High variance clusters account for only $4 \%$ of $\mathrm{DE}$ genes, but belong to 11 clusters with the highest rates of variance (see Chapter II, Figure II- $4 \mathrm{~b}$, green). The highest degree of variance is shown by Cluster 12, which consists of only two genes: 254916 and 16824 
(Table S1). A closer look at $C$. parasitica genomic sequence reveals that these two genes are very close together, separated by a $1000 \mathrm{bp}$ spacer. I merged the exon sequences of both genes together and performed a thorough BLAST search against non-redundant NCBI database. Analysis revealed that both sequences show very close similarity to Short-Chain Dehydrogenases (SCD) from fungal plant pathogens. Among BLAST results, the best hit was for SCD from Colletotrichum gloeosporioides (e-value $<10^{-100}$ ). However, more functionally meaningful annotation came only after we analysed sequence against UniProt-SwissProt database. The first gene carries close ortholog of Tetrahydroxynaphthalene reductase (UniProt ID: Q12634, e-value $<10^{-11}$ ) from a fungus Pyricularia oryzae. The second is an unidentified gene that shows homology only to uncharacterised fungal proteins. BLAST analysis against UniProt-SwissProt database of both genes together showed the same result, a match to Tetrahydroxynaphthalene reductase (e-value $<10^{-10}$ ). Most importantly, however, expression values of both genes are nearly identical. This allows us to suggest that these two sequences may be exons of one single gene, expressed and controlled by the same upstream promoter. The reason they were annotated as separate genes in C. parasitica genome is because the $1000 \mathrm{bp}$ spacer region was showing a lack of transcript accumulation. The most likely explanation for that large gap in the sequence alignment is the high sequence polymorphism within

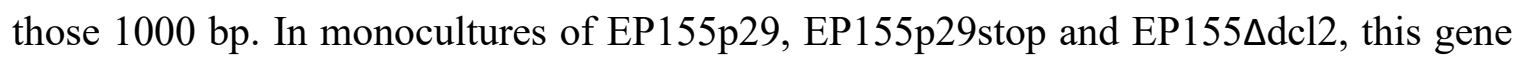
shows significant downregulation compared to control wild-type strain. In fact, in EP155p29stop both genes showed no transcript accumulation at all. Similarly, in P74-3 only 254916 showed weak transcript accumulation, compared to other monocultures. During barrage, Tetrahydroxynaphthalene reductase (represented by genes 254916 and 


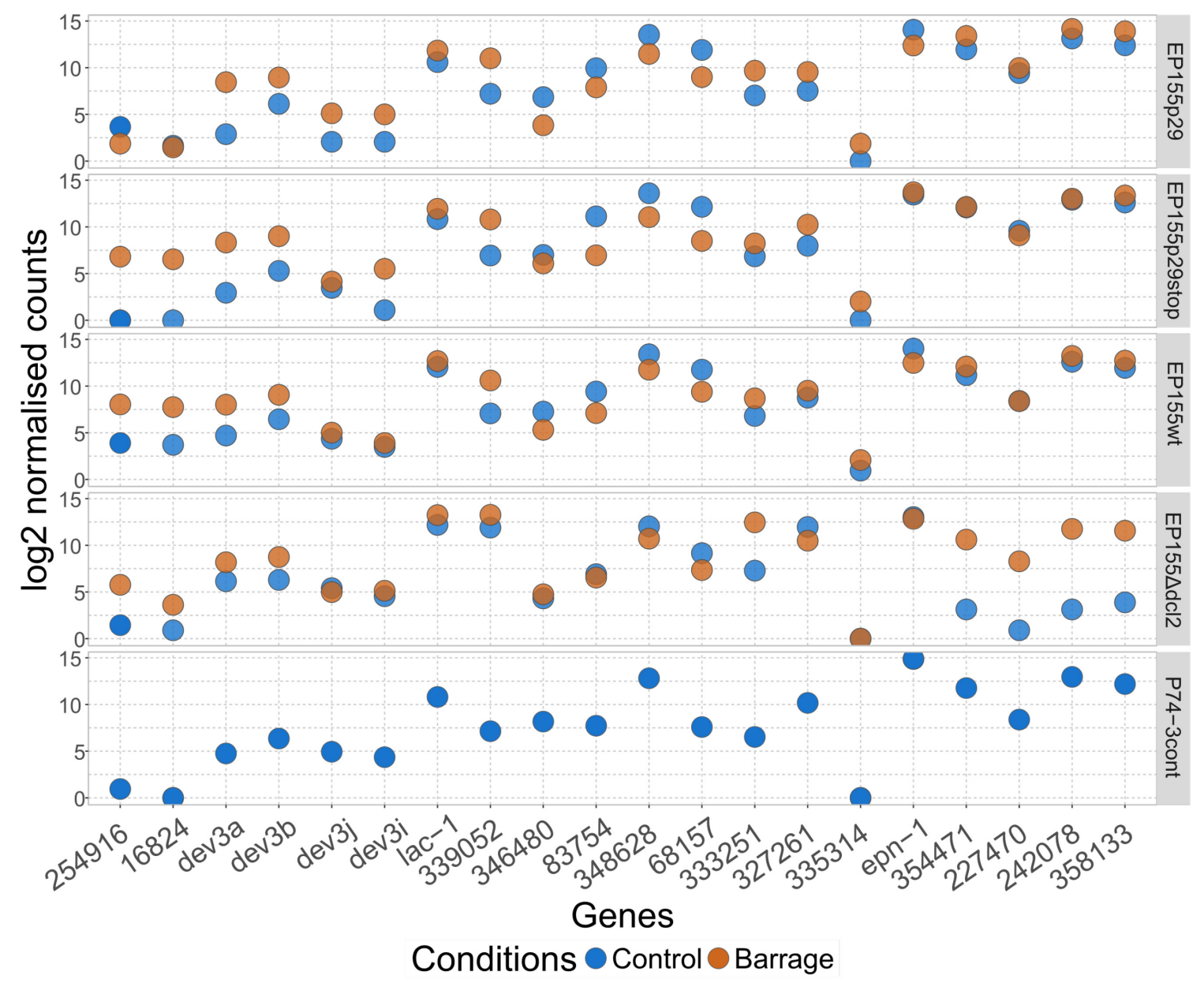

Figure III - 2. Normalised expression rates of Tetrahydroxynaphthalene reductase, HET domain genes (dev3 abij), laccase (including lac-1 and five probable laccases) and endothiapepsin genes during vic 3 incompatibility.

Y-axis of the graph shows estimated read counts from RNA-seq analysis, normalised and transformed on $\log 2$ scale. X-axis genes description: 254916 and 16824 - two exons of tetrahydroxynaphthalene reductase; $\operatorname{dev} 3 a$ (ID: 261856), $\operatorname{dev} 3 b$ (ID: 262887), $\operatorname{dev} 3 j$ (ID: 53104) and dev3i (ID: 243920) - HET-domain genes DE during vic3 incompatibility; lac-1 - laccase 1 gene (ID: 69386); Gene IDs: 339052, 346480, 83754, 348628, 83754, $68157,333251,327261$ and 335314 are probable laccase genes; 
16824) is upregulated in parings of P74-3 and either EP155wt, EP155p29stop, or EP155 ddc12 - compared to respective control monocultures. But in presence of p29 in barraging samples Tetrahydroxynaphthalene reductase shows no differential expression from monoculture expression rates. This indicates that p29 inhibits activation of Tetrahydroxynaphthalene reductase during barrage (Figure III-2 and Table S1).

Tetrahydroxynaphthalene reductase is a well-studied protein catalyzing two steps on the pathway to melanin production (Vidal-Cros et al. 1994). Melanin can be seen as an important virulence factor, as it is found to be a functional component of tissue invasion structure called appressorium. For example, in the rice blast fungus, $P$. oryzae, melanin is responsible for turgor regulation, allowing the pathogenic fungus to use the appressorium to puncture host rice plant cells (Bechinger et al. 1999). Any disruptions in melanin biosynthesis may reduce the invasiveness of $P$. oryzae, and for that reason, Tetrahydroxynaphthalene reductase is commonly used as a target for fungicides (Liao et al. 2001). To form cankers and spread within the Chestnut tree host, C. parasitica faces similar obstacles as rice pathogen $P$. oryzae. To spread and multiply $C$. parasitica forms stromata that will develop into structures called perithecia (fruit-bodies) and pycnidia (asexual organs that produce conidia). As a result, $C$. parasitica must be able to puncture through thick chestnut bark to aid in dispersal of spores. Getting spores out helps the fungus to effectively spread through the aid of animal vectors or wind. So far, there has been no research on a role for melanin in C. parasitica, but presumably, melanin may be important in appressorium-associated infection of the chestnut host. However, host penetration in $C$. parasitica was previously attributed to cryparin, a fungal hydrophobin, mostly expressed in perithecia (Kazmierczak et al. 2005). Similarly, hydrophobin has 
been identified to play a crucial role in appressorium function in P. oryzae (Talbot et al. 1993). Further, it was previously shown that the cryparin gene is downregulated in CHV1 infected strains (L. Zhang et al. 1994). According to our data, however, cryparin expression was stable in all experimental conditions, showing no differential expression during barrage or in presence of p29 in C. parasitica. Lack of p29 effects on cryparin mRNA accumulation may indicate that other parts of CHV1 genome are responsible for its regulation. Alternatively, lack of p29 effect may be explained by growth conditions. For example, previous data indicated cryparin is the most abundant protein when $C$. parasitica cultures are grown in liquid media (Kazmierczak et al. 2005). As all cultures in this study were transferred from liquid medium to solid medium, we can suggest that overexpression and activation of cryparin in reaction to liquid medium may overshadow any modulations by the virus.

Overall, we suggest that 'genes' 254916 and 16824 encode a single reductase gene responsible for the production of a melanin-like compound(s). This in turn may regulate turgor pressure, most probably in stromata, to penetrate plant tissue. In that case, the inhibition of Tetrahydroxynaphthalene reductase by p29 may be a main contributor to distinct canker morphology of hypovirulent $C$. parasitica strains. If true, this would suggest that barrage elicits a similar response in C. parasitica as during pathogenic interactions with the Chestnut host.

It is hypothesized that a main function of barrage is to restrict transmission of parasitic genetic elements during strain contact in nature. Previous studies demonstrate that the rate of virus transmission negatively correlates with the rate of cell death during barrage (Biella et al. 2002). Some mycoviruses develop effective adaptations, like 
Sclerotinia sclerotiorum mycovirus, which can completely inhibit allorecognition allowing for unlimited transmission (S. Wu et al. 2017). Microscopy analysis shown in Figure III-1, demonstrates that the death rate between incompatible strains expressing p29 protein is almost two times lower than in the wild type and p29stop control strains. While CHV1 has never been shown to completely inhibit barrage formation in $C$. parasitica, it may reduce the PCD rate that occurs during the barrage process, thus facilitating virus transmission (Biella et al. 2002). Our main attention from here will focus on genes whose expression was affected by p29 in both control and barraging samples.

With respect to barrage mechanisms, of special interest are genes that encode a HET domain, since this domain has been identified as a genetic determinant of fungal allorecognition (Smith et al. 2000). Previous studies on het-R/het- $V$ induced heterokaryon incompatibility in $P$. anserina demonstrate that almost $50 \%$ of all identified HET genes increase in expression (Bidard et al. 2013). This DE in a large proportion of HET-domain genes may be somewhat context dependent since only five HET domain genes were DE in similar experiments during het-c/pin-c incompatibility in N. crassa. In our dataset (see Table S1), out of 124 known HET-domain genes in the C. parasitica genome, only seven were differentially expressed during vic3-associated barrage formation involving EP155 and P74-3 (six upregulated, one downregulated). The expression levels of two other HET-domain genes $\operatorname{dev} 3 a$ and $\operatorname{dev} 3 b$ (IDs: 261856, 262887) showed higher expression in strains in barrages involving EP155p29 and EP155p29stop (Figure III-2). A closer look at absolute expression values reveals that higher $\mathrm{DE}$ in barraging samples is not a result of higher expression of these latter two HET genes during barrage, but rather the lower 
transcript levels in control strains expressing p29 or p29stop. Basically, during barrage all activated HET genes have almost the same expression rate, but control strains expressing

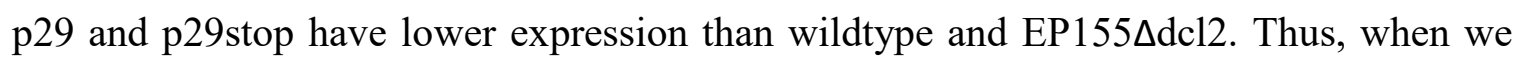
subtract control values from barraging samples, barraging samples with p29 and p29stop produce larger DE value. This clearly indicates that p29 and p29stop cannot influence the expression of HET genes when barrage is activated, though they affect transcript accumulation for various HET genes at initial conditions. Downregulation of HET genes during the initial phases of PCD, after incompatible strains fuse, may play a role in reducing the rate of $\mathrm{PCD}$. If $\mathrm{CHV} 1$ or, in our case, $\mathrm{p} 29$ protein would be able to completely block expression of HET genes, this would make strains compatible, as it happens when HET genes are knocked out (G. H. Choi et al. 2012; D. X. Zhang et al. 2014). If a slight downregulation of HET genes expression delays the initiation, and slows down the rate, of PCD during barrage, this may increase the time window for CHV1 transmission after anastomosis occurs. This allows us to conclude that the total inhibition of barrage may not be necessary for effective mycovirus transmission.

If p29 is able to increase transmission of CHV1 by reducing the expression of HET genes, we can expect that virus can influence HET genes related to several types of incompatibility. In addition to seven HET genes activated during barrage, it is interesting to see other HET genes downregulated in control samples. In our dataset, we identified two HET genes dev3i and dev3j (IDs: 53104, 243920) downregulated in EP155p29 control strain but not in EP155p29stop. These genes are not differentially expressed during barrage induced by vic3 incompatibility, and were never identified in previous studies to be involved in allorecognition in C. parasitica. Incompatibility by vic3 
activates several HET domain genes (discussed further in Chapter IV) even though genes at the vic3 locus do not contain HET domains. Here we can hypothesize that HET genes 53104 and 243920, similar to HET genes activated by vic3, may play a role in other types of allorecognition which subsequently affect CHV1 transmission.

Previous studies on how CHV1 influences expression of particular C. parasitica genes did not evaluate what happens during barrage formation. As our data demonstrates, however, influence of p29 on certain genes in monocultures and barraging strains can be largely different. Historically, one of the first genes that was shown to be altered in $C$. parasitica by CHV1 was laccase-1 (lac-1, protein ID: 69386) (G. H. Choi et al. 1992; Larson and Nuss 1994). Initial work showed that the presence of CHV1 inhibits expression of lac-l even in the presence of the chemical inducer of laccase transcription, cycloheximide. Our transcriptome data does not support previous observations that expression of lac-1 in C. parasitica is decreased when p29 or p29stop are present. Furthermore, lac-1 transcript abundance did not change in barraging strains either. On the other hand, there are at least nine predicted laccase genes which show significant changes in expression. Only one of these laccase genes (ID: 342366) was affected by p29. It showed no change in expression during barrage compared to controls, but was significantly downregulated in EP155p29 monoculture strain compared to EP155wt control. The other eight probable laccase genes do not demonstrate any consistent DE patterns although some were affected by barrage, either up- or downregulated (339052, $346480,83754,348628)$. Thus, data on laccase genes expression is inconclusive and it is hard to predict any possible phenotypic outcomes from transcription data. It is certain that analysis of Laccase protein activity is necessary to provide final conclusions on p29 
effects. Additionally, studies mentioned in Chapter I indicate other regions of CHV1 genome that can influence $C$. parasitica phenotype (see Figure I-6). Thus, presence of full-length CHV1 genome may present different expression profile in relation to laccases genes.

Interestingly, out of 9 probable laccase genes five of them (IDs: 83754, 68157, $333251,327261,335314$ ) change their expression (either up or down) due to the Dicer-2 deletion in comparison to EP155wt. The Laccase 83754 mentioned above is downregulated in monoculture of EP155 ddc12 compared to EP155wt, and downregulated in barraging stains compared to monocultures. Among the other four probable Laccase genes, 68157 is downregulated in EP155 ddc12 as well, compared to EP155wt. The other

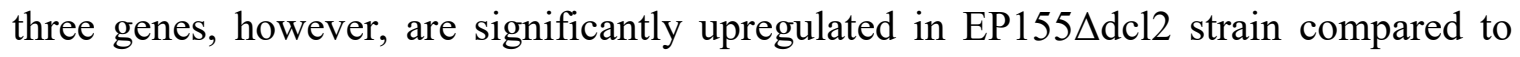
wildtype. This may imply that the expression of these Laccase genes is controlled via transcript degradation by upstream Dicer-2-dependant RNAi processes.

Another classic example of a gene that is effected by CHV1 in C. parasitica is endothiapepsin, epn-1. In the initial work by G. H. Choi et al. (1993), it was reported as unaffected by $\mathrm{CHV} 1$, while in a later analysis it was reported to be downregulated during CHV1 infection (Todd D. Allen et al. 2003). In our dataset, expression of epn-1 (ID: 100383) did not differ in the control EP155wt strain and EP155p29, but were able identify close to significant downregulation (1.97 and 2.09 on $\log 2$ fold scale) in

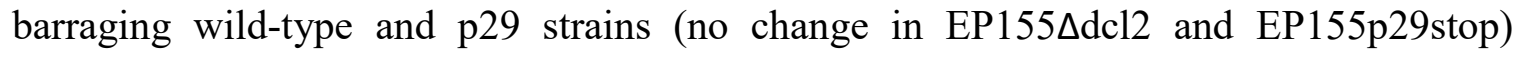
compared to EP155 and P74-3 monocultures. In addition, we identified four probable paralogs of epn-1. These include putative endothiapepsin genes (IDs: 354471, 227470, 242078, 358133) that are homologous to Aspergillopepsin-2. These genes are 


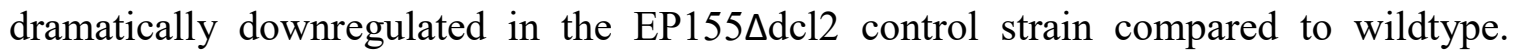
When we estimate differential expression in barraging strains, we see that all Aspergillopepsin-2-like genes show similar expression profiles. In barraging samples all the genes show similar expression rate, but in control monocultures some are downregulated. The situation is similar to what was described above for HET genes. This leads to the apparent high DE values during barrage of P74-3+EP155 $\Delta \mathrm{dcl} 2$ barraging

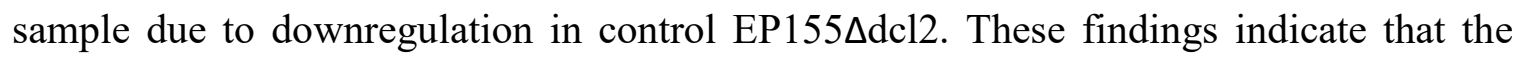
absence of Dicer-2 activity affects expression of endothiapepsin genes primarily in control conditions. Regardless, it is unclear what makes Aspergillopepsin-2-like genes so dependent on Dicer-2 expression in C. parasitica.

Among genes affected by p29 in monocultures, but not in barraging samples, one presents particular interest in relation to the CHV1 life cycle. Previous work found that CHV1 depends on stimulation of trans-Golgi network vesicle formation, where p29 is integrated into the vesicle membrane (Jacob-Wilk et al. 2006). Our data indicates that expressed p29 may possibly stimulate vesicle formation by promoting the expression of a homolog of SEC13, which encodes part of the protein complex involved in ER vesicle formation in yeast (Pryer et al. 1993). The SEC13 homolog in C. parasitica (ID: 73939) is overexpressed in EP155p29 and EP155p29stop strains compared to EP155 wild-type, and its expression pattern is not altered in barraging strains (Table S1). Once again, we see that the presence of p29 mRNA is enough to cause gene overexpression. 


\section{Conclusion}

In this chapter, we demonstrate the effects of p29 protein from CHV1 virus on gene expression in $C$. parasitica strains and during barrage formation in vic3-incomaptible pairings. Fluorescent microscopy analysis shows that the expression of p29 protein can reduce cell death rates during early stages of barrage development. We have considered previous observations which have shown that CHV1 transmission rates significantly increase when cell death rate goes down (Biella et al. 2002). This data suggests evolutionary pressure on CHV1, pushing it toward developing mechanisms to inhibit barrage. At the same time, all EP155-derived strains used in the current work, including the one that expresses CHV1-p29, are able to form clear barrages with the vic3incompatible P74-3 strain. Thus, CHV1 does not inhibit barrage outright, but does appear to reduce the rate of PCD at the beginning of barrage development. As mentioned above, some HET genes are downregulated by p29 and downregulation of HET genes in control strains by p29 may prevent normal accumulation of HET domain proteins when barrage is activated. This allows us to suggest a possible mechanism by which CHV1 can delay onset of barrage. In general case, $\mathrm{CHV} 1$ virus downregulates some HET genes in infected strain, so that when the strain barrages with incompatible counterpart low transcript levels of HET genes slows down activation of cell death. As both strains have to fuse first before incompatibility reaction can start, downregulation of HET genes may create an extended time window for CHV1 to transfer to another strain. Further discussion on p29 and HET genes can be found in Chapter IV.

In addition, we have shown that downregulation Tetrahydroxynaphthalene reductase by p29 may present a significant effect on fungus virulence. Following the 
analogy of the rice blast fungus, Pyricularia oryzae (Liao et al. 2001), we infer that inactivation of this gene may result in reduced canker size on chestnut tree. Similarly fungicides (tricyclazole, phthalide, pyroquilon) that inactivate Tetrahydroxynaphthalene reductase in P. oryzae (Liao et al. 2001) may as well be effective on C. parasitica to reduce canker size.

From an evolutionary standpoint, chestnut blight presents a particular interest, as an example of three-species coevolution. Developing a balance in mutual coexistence of three species, chestnut tree, C. parasitica, and CHV1, becomes more evident if we bring the context of data presented above. Downregulation of HET genes helps CHV1 to spread more effectively from infected to uninfected C. parasitica strains. Inactivation of Tetrahydroxynaphthalene reductase hypothetically helps the virus to reduce damaging effects of C. parasitica on its host. Chestnut is able to survive infection with hypovirulent C. parasitica strain, but it cannot eliminate fungus completely. At the end, reduced $C$. parasitica virulence allows chestnut trees to survive, and in turn increases the life-span and therefore reproductive period of all three organisms. From that perspective it is possible to suggest that despite wide pleiotropic effect of p29 on molecular pathways in C. parasitica, only few genes may play a crucial role in affecting fungal virulence and virus transmission. 


\section{CHAPTER IV. \\ Expression profile of HET genes during allorecognition in Cryphonectria parasitica.}

\section{Introduction}

Programmed Cell Death (PCD) in fungi was first studied in Saccharomyces cerevisiae (Frank Madeo et al. 1997). In yeast, PCD can be elicited by various stress factors such as Reactive Oxygen Species (ROS), toxins, and UV light exposure (Shlezinger et al. 2012). The molecular mechanisms of PCD in yeast are similar to those described for animals that demonstrate signs of caspase-dependant and caspaseindependent types of apoptosis (Carmona-Gutierrez et al. 2010). However, yeast apoptosis lacks many crucial apoptotic regulators found in animals. For example, homologs of Bcl-2 protein family members have not been identified in yeast, or in other fungi (Shlezinger et al. 2012). Most studies conclude that the mechanisms of apoptosis in yeast is intrinsic by origin. This means that in the centre of molecular pathway leading to yeast PCD lies in proteins released from mitochondria such as Cytochrome $c$, caspase-9 and apoptosis inducing factor (AIF) (F. Madeo et al. 2002; Wissing et al. 2004). One of the surprising findings in yeast death studies was a discovery that sexual pheromones can induce PCD (Pozniakovsky et al. 2005). Observations conclude that in mixed cultures of sex types, $\sim 6 \%$ of cells showed signs of PCD. With higher pheromone concentrations, the death rates go up to $30 \%$ (Severin and Hyman 2002).

Further studies indicated that pheromone induced death lacks the main features of apoptosis death and as a result, was more connected to external signaling (N. N. Zhang et al. 2006b). In particular, cell death was associated with Fig1 transmembrane protein 
receptor and cell wall degradation. Fig1 is related to the Claudin protein family that regulates epithelium permeability in animals (Harris et al. 2010). In general, it was suggested that pheromone-related cell death is the result of failed mating. When a yeast cell is exposed to the opposite mating pheromone it triggers processes related to shmoo formation, but without a mating partner around the same processes result in cell death (Maeder et al. 2007; N. N. Zhang et al. 2006b).

A key component of HI-associated allorecognition are incompatibility loci, many of which are characterised by the presence of a HET domain (Smith and Lafontaine 2013). NACHT (and corresponding protein clade), and WD or LLR are additional two domains commonly associated with incompatibility loci (Fedorova et al. 2005; Paoletti and Saupe 2009). Genes that have complex domain structure, carrying HET, NACHT and WD belong to the class of proteins called the signal transduction ATPases with numerous domains (STAND). This is a broad group which among others includes proteins involved in innate immunity response (Leipe et al. 2004). The NACHT domain is commonly found in P. anserina proteins responsible for allorecognition (Paoletti et al. 2007). Such genes in $P$. anserina usually also include a HET domain at the N-terminus and WD repeat at the C-terminus. The mode of action of such gene products was suggested to involve HET domain as a prime activator of $\mathrm{PCD}$, which is in turn kept deactivated through the WD-repeat domain. When signal molecules, like parts of bacterial cells, appear in the cytoplasm of the fungus they bind to the WD-repeat and activate HET domain (Paoletti and Saupe 2009). In this system, WD-repeat evolves to recognise specific molecular signals and NACHT is a NTP-binding and oligomerization domain. So far, the HET domain has been identified almost exclusively in fungi and has an unknown 
molecular function. The only certainty is that HET genes, by some means, activate incompatibility and downstream PCD processes (Smith and Lafontaine 2013). Although not all recognised HI and vic loci do contain genes encoding a HET domain, a study with P. anserina showed that during induced incompatibility about $50 \%$ of the 130 HETdomain genes identified in the genome are overexpressed (Bidard et al. 2013). This suggests a significant level of redundancy in the allorecognition system, which may involve activation of several HET-domain genes in addition to the subset that was previously associated with incompatibility.

In this chapter we make a detailed examination of transcription data from Chapter II. We perform an additional set of tests to verify and expand on RNA-seq expression data. Close analysis of differentially expressed genes allowed the creation of a more detailed model of key processes activated during barrage formation in vic3 incompatible strains of $C$. parasitica. This model will serve as a useful guide for further dissection of vic-associate PCD in C. parasitica in the future.

\section{Material and Methods}

Reverse transcription from RNA template was performed using M-MuLV Reverse Transcriptase (New England Biolabs, Whitby, ON, Canada) according to manufacturer recommendations. Real-time quantitative PCR analysis was performed using a CFX Connect Real-Time PCR Detection System (BioRad, Mississauga, ON, Canada) with KAPA SYBR FAST Universal 2x master mix (KAPA, Wilmington, MA, USA). Gene expression values were normalised against 18S rRNA (forward primer: 5'AtAACAGgtCtGtGATGCCCTTAGA-3' (T. B. Parsley et al. 2002); reverse primer: 
5'- CAGGGACGTAATCAACGCAAG-3') or gpd1 (glyceraldehyde-3-phosphate dehydrogenase; forward primer: 5'-GCCTACATGCTCAAGTATGACTC-3'; reverse primer: 5'-AAGACACCAGTGGACTCGACAAT-3'). Relative abundance of normalised transcripts was calculated using 2- $\Delta \Delta \mathrm{Ct}$ method (Livak and Schmittgen 2001). Real-time PCR primers used for measuring transcript abundance of selected C. parasitica genes are given in Table IV-1.

For other materials and methods used in this chapter see Materials and Methods section in Chapter II. 
Table IV - 1. Primers used for quantitative reverse polymerase quantitative chain reaction (RT-qPCR).

\begin{tabular}{|c|c|c|c|}
\hline Primer names & CpID $^{2}$ & Sequence $\left(5^{\prime}-3^{\prime}\right)$ & $\begin{array}{l}\text { Reference and } \\
\text { purpose }\end{array}$ \\
\hline & \multicolumn{3}{|c|}{$\begin{array}{ll}\text { Reference genes } \\
\end{array}$} \\
\hline $18 \mathrm{~S}-\mathrm{F}$ & & ATAACAGGTCTGTGATGCCCTTAGA & $\begin{array}{l}\text { (T. B. Parsley et al. } \\
\text { 2002), qPCR }\end{array}$ \\
\hline \multirow{3}{*}{$\begin{array}{l}\text { 18S-R } \\
\text { gpd1-RTQ2-F } \\
\text { gpd1-RTQ-R }\end{array}$} & \multirow{3}{*}{101864} & CAGGGACGTAATCAACGCAAG & \multirow{3}{*}{ This study, qPCR } \\
\hline & & GCCTACATGCTCAAGTATGACTC & \\
\hline & & AAGACACCAGTGGACTCGACAAT & \\
\hline \multirow{11}{*}{$\begin{array}{l}\text { Mf2-1 RTQ - F } \\
\text { Mf2-1 RTQ - R } \\
\text { Mf1-1 RTQ - F } \\
\text { Mf1-1 RTQ - R } \\
\text { MAT-1 RTQ - F } \\
\text { MAT-1 RTQ - R } \\
\text { MAT-2 RTQ-F } \\
\text { MAT-2 RTQ-R } \\
\text { cpVIB1-RTQ-F } \\
\text { cpVIB1-RTQ-B }\end{array}$} & \multirow{3}{*}{85578} & \begin{tabular}{|l} 
Mating genes \\
\end{tabular} & \multirow{11}{*}{ This study, qPCR } \\
\hline & & AATGCCTTCCAACACCCAGAC & \\
\hline & & GCTCTTATGGCGTGGGCTG & \\
\hline & \multirow{2}{*}{333020} & CCTGAGGCTTGGTGTCTCTT & \\
\hline & & GAACTCAGGAGGCTCCGAG & \\
\hline & & ATCTTGCTGGCACCATCTGT & \\
\hline & \multirow{3}{*}{44005} & CATTCTCGAGGGGCAGTCCA & \\
\hline & & GGTCGAAGCAGGAGTGGAA & \\
\hline & & CGACCCCTTCACTGGAGCTTAC & \\
\hline & \multirow{2}{*}{67224} & CCATCGTCCCTACCGCTCA & \\
\hline & & TTCTCCСТCTCTACCGCTTG & \\
\hline \multirow{9}{*}{$\begin{array}{l}\text { dev3a-RTQ-F } \\
\text { dev3a-RTQ-R } \\
\text { dev3b-RTQ-F } \\
\text { dev3b-RTQ-R } \\
\text { dev3g-RTQ-F } \\
\text { dev3g-RTQ-R } \\
\text { vic1a-RTQ-F } \\
\text { vic1a-RTQ-R }\end{array}$} & \multirow{3}{*}{261856} & $\begin{array}{ll}\text { HET genes } \\
\end{array}$ & \multirow{9}{*}{ This study, qPCR } \\
\hline & & GCACCCCAATTTTGAGTTCTCC & \\
\hline & & GCACTCTCTCCATTGCCCAAG & \\
\hline & \multirow{2}{*}{262887} & CAGCCCAGGACATTAGAGACA & \\
\hline & & TCGGTCCGTATAGTACAGCTC & \\
\hline & \multirow{2}{*}{240373} & CCATGAGAACGCCTACATGACA & \\
\hline & & CTCCTCACGCAAATCTCGTCT & \\
\hline & \multirow{2}{*}{258862} & TCGCTCTGCATTATCCAGGAC & \\
\hline & & TCTATGTCGCCATCACCAC & \\
\hline
\end{tabular}

${ }^{2} \mathrm{CpID}$ - protein IDs provided for primers which were designed in this study based on EP155 sequence from JGI C. parasitica genome project. 


\section{Results and Discussion}

Allorecognition in filamentous fungi is associated with rapid activation of Programmed cell death (PCD) (Biella et al. 2002; Jacobson et al. 1998). The molecular mechanisms of recognition and the signal transduction that ends in cell death remain largely unknown. Previous observations showed that morphological characteristics of PCD has similarities to apoptosis, including nuclear fragmentation, DNA degradation and reactive oxygen species (ROS) production (Carmona-Gutierrez et al. 2010; Fedorova et al. 2005). Other investigations indicate that molecular mechanisms of PCD in filamentous fungi may involve different molecular pathways compared to well-studied apoptosis models in yeast or mammals (Hutchison et al. 2009; Shlezinger et al. 2012). If compared to animal or insects models of PCD, it becomes obvious that our current understanding of PCD in fungi is very fragmented.

In the previous chapter we characterized overall transcriptional background in vic3-incompatible strains. Here, we provide a more detailed view of the molecular processes associated with PCD during allorecognition in C. parasitica. For clarity purposes, I divided processes associated with barrage formation into four consecutive phases: 1) hyphal fusion; 2) incompatibility trigger; 3) PCD pathway; 4) final stages of cell death. However, in the following sections I decided to present these phases in reverse order. Thus, in the first section I deal with sexual reproduction genes and give an idea of the final outcome of barrage-associated PCD. Sections on oxidative stress and vegetative incompatibility form the core to this chapter. Here, analyses of genes related to oxidative stress shed light on the PCD pathway that is triggered by incompatibility genes. 
In the final section of this chapter, I present possible connections of incompatibility genes and PCD to processes of hyphal fusion.

\section{Sexual reproduction genes.}

Programmed cell death associated with sexual reproduction in fungi was initially described for yeast as a result of failed mating. Additionally, in P. anserina it was shown that deletion of metacaspases genes leads to defects in ascospore formation (Hamann et al. 2007). Similarly, the plant pathogen Coniochaeta tetrasperma shows involvement of PCD in ascospores development (Raju and Perkins 2000). Further, our observations indicate that pheromone expression drastically increases as a result of vic3 incompatibility (Figure IV-2). As was mentioned in Chapter II, pheromone precursor genes $m f 2-1$ (ID: 85578) and $m f 2-2$ (not annotated in C. parasitica genome, last identified as novel gene NOV_010635) demonstrated the highest rate of differential expression (DE) in the entire sample set. This upregulation of $m f 2-1$ and $m f 2-2$ is explained by very low abundance of these transcripts in control strain in comparison to very high abundance in barraging strains. Basically, in control strains, the absolute expression is 1, but during barrage, the expression is 14 on $\log 2$ scale. This observation was surprising for three reasons. First, previous studies indicate that pheromone genes are constitutively expressed (Turina et al. 2003; L. Zhang et al. 1998), whereas we provide evidence that pheromone genes are regulated during vic3-associated incompatibility. A second surprising result comes from EP155p29 strain, where p29 did not downregulate pheromone gene expression as expected. Previous studies indicated that main hypovirulence symptoms caused by Cryphonectria hypovirus (CHV) infection are determined by p29 protein (see Figure I-6, Chapter I). Although, there is no direct 
evidence that p29 can influence pheromone genes expression, strains infected with CHV show decreased $m f 2-1$ and $m f 2-2$ expression (L. Zhang et al. 1998). At the outset we had thought that expressed p29 would mimic CHV infection and decrease the expression of pheromone genes. Our data, however, indicated no differences in pheromones expression in EP155p29 and EP155p29stop strains compared to wild-type. Low expression of pheromones might not allow us to detect differential expression in monocultured controls strains, but significant differences in pheromone genes expression were not observed between barraging strains compared to each other. Third, and finally, the drastic increase in expression of genes involved in sexual reproduction during vegetative incompatibility is unexpected given that vegetative incompatibility is not considered part of the sexual cycle. We would not expect sexual reproduction signaling during interaction between EP155 and P74-3 given that these strains are both MAT-2. In addition, previous studies on transcriptional profiles during induced heterokaryon incompatibility (HI) in N. crassa and $P$. anserina did not identify any DE genes directly associated with sexual reproduction (Bidard et al. 2013; Hutchison et al. 2009), except that in N. crassa increased transcript levels were noted for $p p-1$, an ortholog of the yeast transcription factor Ste12, and mak-2 protein kinase (Hutchison et al. 2009). These two genes are part of mating signaling and have identified orthologs in C. parasitica genome (E. S. Choi et al. 2005; Deng et al. 2007). In our analysis the C. parasitica orthologs of Ste12 and mak2 did not show differential expression during vic3 incompatible interactions.

The observed low levels of pheromone expression in our monocultured samples may be explained by growth conditions that were used. Previous studies on $m f 1-1$ pheromone precursor gene, reveal that strains grown in liquid media lack pheromone 
expression, and transcript accumulation is detectable only after a week of growth on agar media (Turina et al. 2003). As described above, in our barrage setting, strains were treated with Trichoderma lysing enzyme before plating on agar medium to create an even distribution of barraging hyphae. Considering that RNA extractions were performed on days 2 and 3 after plating onto agar medium, we can surmise that within this time span, after a transfer from liquid to solid agar media, transcriptional activity of pheromone genes remains similar to conditions in liquid medium. Considering the above, pheromone gene expression may be more of a natural part of the mycelial aging process in which case we can suggest that allorecognition increases the rate of mycelial maturation or an aging process. Interestingly, the same study demonstrated that along with pheromone expression, strains in liquid media do not produce conidia (Turina et al. 2003). Similarly, our observations show that strains in monocultures lack conidia until day 7 of growth on agar medium. However, barraging samples start to produce conidia during 4 to 5 days of growth. It is therefore possible that activation of pheromones is associated with activation of asexual sporulation along with PCD.

Not much is known on how and why pheromones are activated during conidiation. It is certain, however, that upstream activation of pheromone genes depends on activation of mating type genes (Bistis 1981; Kües and Casselton 1992). The mating system in $C$. parasitica is similar to other heterothallic Pyrenomycete fungi (McGuire et al. 2001). The mating-type locus can harbour one of two possible idiomorphs, mat-1 or mat-2, which determines the sex of the mycelium. This means that homozygous individuals will carry only one of those idiomorphs. In turn, MAT genes regulate expression of three mating 
pheromone genes, $m f 2-1$ and $m f 2-2$ are activated by the MAT-2, and $m f 1-1$ are activated by MAT-1 (McGuire et al. 2001).

Here, we confirmed overexpression of pheromone genes in vic3-incompatible and other vic-incompatible strain combinations using qPCR analyses. In RNA-seq analysis (see Figure II-3, Chapter II) differential expression by $m f 2-1$ and $m f 2-2$ were the most pronounced of any genes in the data set. High DE was confirmed by qPCR tests with $m f 2-1$ and which we can use as a marker of barrage development (Figure IV-1a,b). Similar to vic3-incompatible pairings involving MAT-1 and MAT-2 strains, both vic1and vic2-associated barrages yield dramatic increases in expression of $m f 2-1$ and $m f 1-1$ (Figure IV-1c). However, vic6- and vic7-associated barrages show moderate increases in $m f 2-1$ and $m f 1-1$ gene expression during barrage. Finally, vic4-incompatible interaction appears to be uncoupled from the mating pheromones. Here, similar to monocultures, pheromones show almost no change in expression on the $3^{\text {rd }}$ day after inoculation of vic4incompatible strain pairs. Based on our earlier interpretation, this suggests that barraging vic4-incompatible strains also do not activate conidiation. This is consistent with previous observations that, out of the six characterized vic loci in C. parasitica, vic4 stands out as having a 'weak' barrage phenotype that allows 100\% CHV transmission (Cortesi et al. 2001) and that does not prevent heterokaryon formation (Smith et al. 2006). 


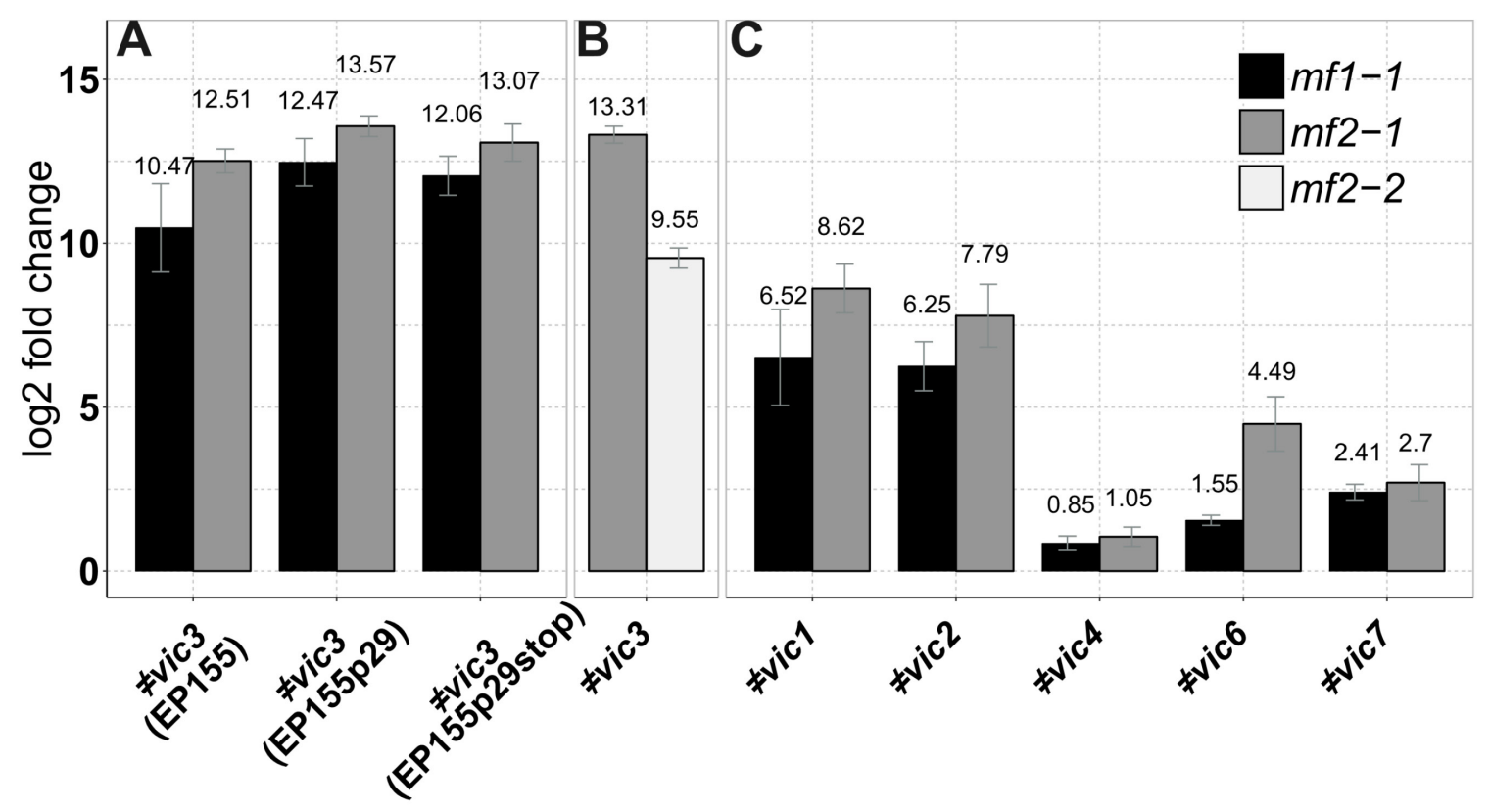

Figure IV - 1. Comparison of differential expression (DE) data acquired by RT-qPCR and RNA-seq of pheromone precursor genes in strains undergoing barrage formation compared to control strains in monoculture.

A) RT-qPCR data for $m f 1-1$ and $m f 2-1$ DE in strains incompatible by vic3 locus. In these pairings EP155wt (MAT-2) and two EP155-derived strains, expressing CHV1 p29 or p29stop were paired with P78-8 (MAT-1) or P74-3 (MAT-2) to detect expression of pheromones for both mating types. Pairings of EP155 strains with P78-8 were used to detect $m f 1-1$ expression and pairings with P74-3 for $m f 2-1$ expression. B) RNA-seq DE data of $m f 2-1$ and $m f 2-2$ (both MAT-2) pheromone genes between vic3 incompatible pairings of P74-3 and EP155. C) DE of mating type MAT-1 and MAT-2 pheromone precursor genes $m f 1-1$ and $m f 2-1$ in interactions of strains that are incompatible due to difference at one of five vic loci. Data presented here for five separate pairing of MAT-2 type strain EP155wt with MAT-1 strains bearing different alleles at one of the vic loci.

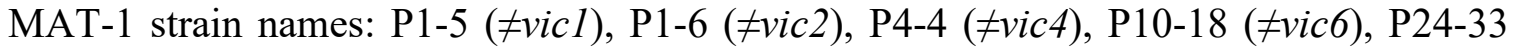
$(\neq v i c 7)$. Whiskers indicate standard error for at least four biological replicas for each gene. 
Apart from mating-type pheromones, there were other genes involved in sexual reproduction that were also influenced by barrage formation based on our RNA-seq analyses. In the RNA-seq analysis described in Chapter II, MAT-2 gene (ID: 44005) demonstrated high differential expression values (see Figure II-4 Cluster 7, Chapter II). RT-qPCR analysis verified this and showed that expression of mat-2 and mat-1 in monocultured strains doubles ( $\log 2$ scale, geometric progression) from day 2 to 3 . However, in barraging strains, expression of mat genes on the second day was already double the value in control monocultures, and it grew much less by the $3^{\text {rd }}$ day. In terms of DE estimation, it creates an effect that, with time, the difference between control and barrage gets smaller. Basically, mat genes appear to be activated earlier than pheromone genes and barrage appears to accelerate mat genes transcript accumulation by about one day compared to levels in monoculture.

A connection between asexual sporulation and activation of genes involved in sexual process has not been observed in filamentous fungi. It is likely that this connection is genus or species specific. A closer look at $C$. parasitica, for example, shows that the life cycle does not involve development of specialised male sexual sporangia. Instead, $C$. parasitica uses asexual conidia as male gametes (Marra and Milgroom 2001). As a result, it is logical to assume that production of conidia may require association with sexual pheromones. However, vegetative incompatibility is not known to be associated with generation of sexual structures. Thus, we can suggest inhibition of the sexual cycle happens downstream of mating genes and pheromones. This indirectly can be confirmed with RNA-seq data. For example, consider cpst12 (ID: 86923), the ortholog of yeast Ste12, a transcription factor required for female fertility (Deng et al. 2007) and prol (ID: 
91188), an ortholog of $N$. crassa transcription factor involved in fruit-body formation (Masloff et al. 2002; Sun et al. 2009b). Previous microarray studies indicated that these genes are both downregulated by CHV1 infection (Todd D. Allen et al. 2003; T. D. Allen and Nuss 2004). This explained one of the main hypovirulence symptom of female sterility and the resulting lack of sexual reproduction within infected populations. In our dataset, neither gene showed significant transcriptional change in presence of p29 or p29stop. This may indicate that their expression is regulated by other factors in the CHV1 genome, other than $\mathrm{p} 29$. In barraging samples cpst 12 was not significantly differentially expressed as well, but prol was slightly downregulated. Previous observation indicates that MAT-2 strains lacking prol expression are unable to produce protoperithecia (Sun et al. 2009b). Alternatively, strains lacking cpst12 expression produce abundant pycnidia (Deng et al. 2007). This is in accordance with typical barrage morphology when dying cells produce abundant pycnidia due to downregulation of prol and active cpst12. This allows us to infer that inhibition of the sexual cycle during barrage most likely happens at the level of sexual organs development (i.e. in fruit-bodies), and downstream of mat and pheromone genes.

In addition, cpste6 (ID: 96416) and cpste11 (ID: 351932) are overexpressed in vic3-incompatible barraging cells. The later of these two genes is an ortholog of the yeast gene Ste $11 \mathrm{p}$, which is involved in the reproductive system. There are actually two yeast genes identified as Stell in the literature. One is MAP kinase Ste11p, a homolog of $N$. crassa NRC1 (cpnrc-1, ID: 66950), which is a component of fusion oscillation complex (Dettmann et al. 2014; Pandey et al. 2004). The second (cpID: 351932, cpstel1) is an ortholog of S. pombe transcription factor Ste11p. For simplicity, from this point on I will 
identify ortholog of transcription factor from S. pombe as Stel1 (cpstel1 as C. parasitica ortholog) and MAP kinase ortholog as $n r c l$ by analogy with $N$. crassa (cpnrcl as $C$. parasitica ortholog). Activation of $n r c l$ ortholog in yeast is a result of the broad spectrum of reactions in response to the presence of pheromones that are detected by regulator of G-protein signaling (RGS) (Dohlman and Thorner 2001). Previous studies showed that G-protein signal transduction through $\mathrm{G} \alpha$-subunit protein CPG-1 and G $\beta$-subunit cpGB-1 is an important virulence factor in C. parasitica (Segers et al. 2004). Interestingly, these studies found that CPG-1 and CPGB-1 are essential for sporulation and pigmentation in C. parasitica and that CPG-1 is important for some types of stress response (S. Gao and Nuss 1996; Segers and Nuss 2003). For example, disruption of the cpg-1 gene makes the fungus more susceptible to oxidative stress and heat shock (Segers and Nuss 2003). Expression of $n r c l$ is not influenced by barrage or p29, but we return to it during discussion on hyphal fusion in relation to barrage formation.

The ortholog of Ste11, on the other hand, is upregulated during barrage. This gene belongs to family of a high-mobility-group (HMG) transcription factors involved in conjugation and activation of meiotic cycle (Qin et al. 2003). In S. pombe, Ste11p is activated in response to starvation or mating pheromones. As a transcription factor it regulates the expression of several genes related to yeast mating, which include mating genes, pheromones and meiosis regulatory factors (Kitamura et al. 2001; Mata and Bähler 2006). In our dataset, we identified two orthologs of yeast genes regulated by Ste $11 \mathrm{p}$ which were also overexpressed during barrage along with cpste11. The first of these genes is a meiotic factor mei2 ortholog (ID: 285012) that was upregulated during barrage regardless of which EP155 variant was used. The second gene regulated by Ste11p in $S$. 
pombe is Ste6 that encodes an ABC-transporter responsible for secretion of a-factor (Kolling and Hollenberg 1994; Sugimoto et al. 1991). The ortholog of Ste6 (cpst6, ID: 96416) in C. parasitica is highly overexpressed in barraging samples. In relation to p29 influence on cpste6, cpste11 or mei2 expression, presence of p29 showed no significant effects. Expression of those genes wasn't affected in EP155p29 or EP155p29stop monocultures in comparison to EP155wt, and it was also uniform in barraging samples. This leads us to suggest that interaction between Ste6p and Ste11p in yeast is, in fact, happening between their orthologs in C. parasitica during barrage formation. Thus, we can conclude that cpSTE6 pumps pheromones into the extracellular environment. It is difficult to derive exact influence of cpST11 on barrage, but we can suggest that $C$. parasitica ortholog is performing a similar function as it is in yeast.

Additionally in connection to sporulation we identified ortholog of $p p o A$ from Aspergillus nidulans. PpoA is a fatty acid dioxygenase that is involved in regulation of balance between anamorph and teleomorph stages of development. Deletion of ppoA causes shift toward increased asexual spore production (Tsitsigiannis et al. 2004). In our data set the ortholog of ppoA (ID: 332509) was significantly downregulated in barraging strains. Downregulation of $p p o A$ provides a mechanism by which the fungus is able to inhibit sexual cycle during barrage and keep mating genes active.

These observations allow us to surmise that expression of pheromones, activated by MAT gene, comes as a component of sporulation signaling. Analyses in Chapter II show that various gene orthologs associated with sporulation in yeast and other fungi are differentially expressed in barraging strains of $C$. parasitica. In addition, studies on Aspergillus showed that conidiation may be caused as a result of various stress factors 
(Adams et al. 1998). On the molecular level, this may indicate a strong connection of asexual sporulation and PCD. For example, it was shown that conidiation in Aspergillus involves caspase activation (Thrane et al. 2004). As well, expression of pro-apoptotic Bax (Bcl-2 protein family), causes increased conidiation in the plant pathogen Colletotrichum gloeosporioides (Barhoom and Sharon 2007). However, it is still not clear: does PCD cause sporulation or does sporulation cause PCD? Our analyses in Chapter II indicated that toxin production is triggered by incompatibility and we posit that this is a probable cause of PCD in C. parasitica. In support of this idea, it was previously shown that PCD during sporulation is associated with toxin production and conidia usually do incorporate secondary metabolites (Thrane et al. 2004). Thus, we can hypothesize that asexual sporulation is a direct result of activated PCD.

\section{Oxidative stress and detoxification}

A notable process inferred to be activated during barrage formation, as described in Chapter II, was related to production of secondary metabolites, including toxins. Another group of processes going in hand with secondary metabolites according to gene enrichment and hierarchical clustering analyses (Figures II-4 and II-5, Chapter II), relates to stress response and detoxification.

Genes related to detoxification occupy the most highly expressed gene clusters. For example, Glutathione S-Transferase (GST) is a member of Phase II detoxification program in animal cells. It is responsible to inactivate reactive electrolytes by binding glutathione (GHS) to them. There are six GST paralogs activated during barrage formation, but two (IDs: 357090, 58765) occupy highly overexpressed clusters 8 and 15 . 
For this study GST is of particular interest because of its role as an anti-apoptotic agent (Circu and Aw 2012). GST action is transcriptionally regulated by transcription factor Nrf2 and presents active defense against xenobiotics and reactive oxygen species ROS (Tew and Townsend 2012). In particular, GST is activated as a defence to inactivate toxins and ROS (Tew and Townsend 2012).

Nrf2 transcription factor activates a battery of anti-stress enzymes in response to redox disbalance or elevated toxicity (Ray et al. 2012). Nrf2 is a transcription factor with high affinity to a gene enhancer, the Antioxidant Response Element (ARE), which is a signature regulator of stress response proteins (Johnson et al. 2008). In our dataset, apart from GST mentioned above, we detected several other orthologs of proteins activated through AP endonuclease 1 (APE) in animal models. Among them, identified in Chapter II, were two Quinone oxidoreductase (QO) orthologs. Activation of QO protects cells from oxidative stress caused by ROS, it helps to maintain redox balance and deactivate toxic quinone derivatives (Jaiswal 2000). Additionally, it was shown that QO is involved in p53-induced PCD in animals (Polyak et al. 1997). Similar functions are carried out by Cytochrome P450 proteins responsible for phase I detoxification, which were discussed in Chapter II as the most enriched protein domain associated with barrage. Here, it should be pointed out that activation of these genes is strongly correlated with toxic environmental stress and is often used as a diagnostic marker of oxidative stress (Uno et al. 2012). In this study, we identified various orthologs of genes involved in Aflatoxin synthesis that were significantly overexpressed in barraging strains. According to previous studies on animal models, metabolism of Aflatoxin depends on $\mathrm{p} 450$ protein activation and its detoxification often involves GST. Aflatoxin metabolism starts in 
microsomes, where it is transformed by Cytochrome p450 to a toxic carcinogenic epoxide that has a strong affinity to DNA and as a result increases mutation rate (D L Eaton and Gallagher 1994; Essigmann et al. 1977; Neal et al. 1986). Inactivation of aflatoxin is suggested to be performed in part by GST, by conjugating the toxic epoxide with glutathione (Eaton and Bammler 1999). Finally, it is worth mentioning that AP endonuclease 1 ortholog (ID: 62735) demonstrated a moderate increase in expression during barrage. APE is involved in DNA damage repair by its endonuclease activity and also uses its DNA-binding domain to play a role as a transcription factor, to activate a variety of other transcription factors in response to oxidative stress (Tell et al. 2008).

Among genes functionally involved in glutathione (GSH) metabolism, apart from GST, were GSH-synthase and GSH-transporter orthologs (IDs: 284549, 97307). Expression of these genes is affected during barrage in all samples. But highest DE was observed for 284549 and 97307 genes in strains lacking Dicer-2 gene expression. GSHsynthase expression was not significantly influenced, but came very close to overexpression threshold in barrages between P74-3 and EP155 ddcl2. Activation of GSHsynthase may lead to increased GSH concentrations in cells, which were found strongly associated with response to Fas/death receptor induced PCD in animal models where there is a release of ROS as a result of mitochondrial membrane permeabilization (Cazanave et al. 2007; Circu and Aw 2012). GSH-transporter, on the other hand, appears

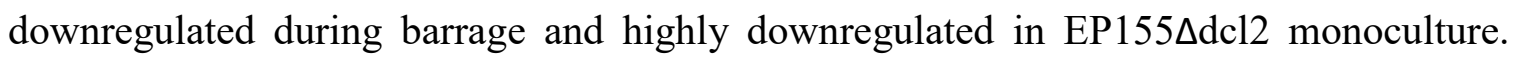
GSH transport inside the cell may lead to more effective detoxification by GST and thus prolong the PCD onset. Therefore downregulation of GSH transporters, along with other 
nutrition transporters (sugar, nitrogen) mentioned in Chapter II, creates more stressful conditions for cells and may accelerate onset of PCD.

Overall, data described in this section so far gives a closer look on processes that were described in Chapter II on a 'global' scale of transcription profile. Increased expression of main Nrf2 transcription factor dependant components (GST, quinone oxidoreductase) of detoxification system and APE may indicate that incompatibility causes rapid increase of ROS production resulting in DNA damage and various toxic effects (Jaiswal 2000; Tew and Townsend 2012; J. Zhang et al. 2006a). This data points to a possible extrinsic nature of PCD related to incompatibility, even when toxins triggering death were produced in the cell.

From that perspective, we can now investigate possible mechanisms of PCD related to processes described above. Looking on cellular level (see Figure I-1, Chapter I) we can see that living parts of interacting incompatible hyphae are protected from dying cells by septa. One of the mechanisms protecting neighbouring cells from PCD-related effects is associated with Woronin bodies (Markham and Collinge 1987). Woronin bodies are modified peroxisomes which plug septal pores and restrict flow of toxic compounds or cytoplasm between cells. Fungi that are unable to produce Woronin bodies due to inactive hex-1 gene, show extensive loss of cytoplasm when hyphae are damaged (Tenney et al. 2000). As studies in N. crassa reveal, Woronin bodies were produced as a result of induced heterokaryon incompatibility (HI), presumably to isolate cells undergoing PCD (Hutchison et al. 2009). Additionally, (Hutchison et al. 2009) identified that Woronin bodies appear at the very early stages of HI, about 5-15 min after induction. However, in our experimental setup we are unable to identify interaction of incompatible 
hyphae at that early stage. Nonetheless, we are able to observe that fused hyphae undergoing PCD were kept separated from neighboring cells. Thus, we can safely assume that Woronin bodies are in fact produced in barraging C. parasitica cells and therefore process should be associated with ROS production.

In Chapter II we proposed that accumulation of ROS may result from mitochondrial membrane permeabilization through extracellular signals (Modjtahedi et al. 2006; M. Wu et al. 2002). This conclusion is based on increased expression of AIF ortholog. To confirm that barraging cells do in fact accumulate ROS, we used the vital dye dichlorofluorescein (DCF) as an indicator of ROS accumulation (LeBel et al. 1992). As shown in Figure IV-2, ROS were identified in the cellular compartments of interacting hyphae, but not in cells around the fusion hyphae.

Activation of GST may serve as an indicator of a very specific type of PCD. As previous studies revealed, GST works as a negative regulator of JNK (Jun-Terminal Kinase); GST binds to and thus maintains low activity of JNK (Ray et al. 2012). However, when redox balance skews towards increased concentrations of ROS, GST dissociates from JNK (Adler et al. 1999). As demonstrated in animals, JNKs are part of external stress activated kinases cascade, which leads to apoptosis through activation of PCD transcription factors like p53 (Davis 2000; Yin et al. 2000). To activate downstream PCD pathway, JNK must be activated by other protein kinases (PKs). Studies showed that an ortholog of yeast Ste20 in animals is responsible for activation JNK (Brown et al. 1996). Ste20 belongs to a group of protein kinases called PAK (p21-activated serine/threonine kinases) that are upstream activators of 


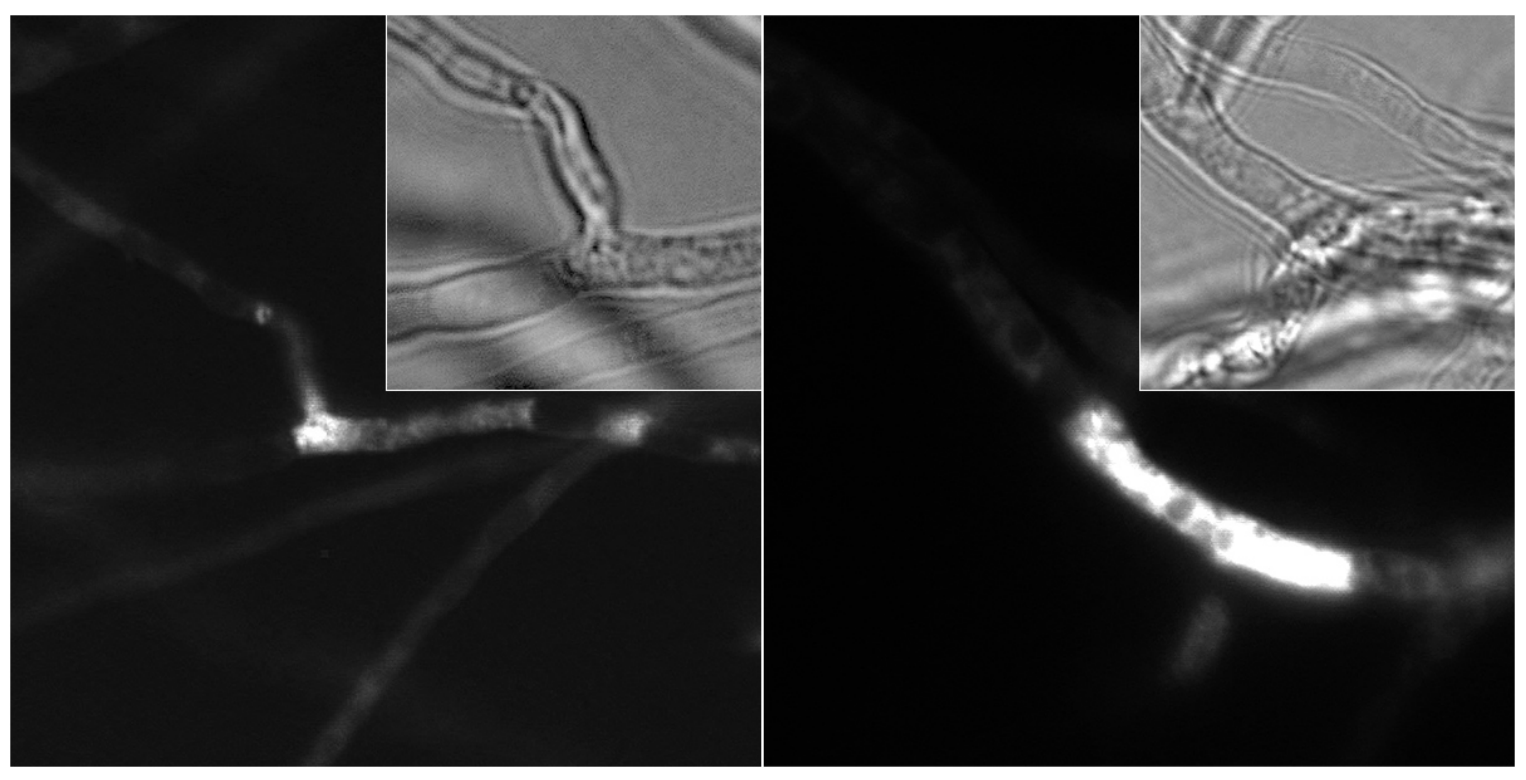

Figure IV - 2. ROS production during vic3 incompatibility.

Strains EP155 and P74-3 were grown for two days on microscope slide and stained with DCF. Fused cells from incompatible strains produce bright fluorescence as a result of ROS production. 
MAPK cascade (Dan et al. 2001). PKs get activated due to a variety of external signals, not necessary related to PCD. Furthermore, association of GST with ASK1 (Apoptosis Signal-regulating Kinase 1) restricts progression of external death signalling in human cells. Similarly to JNK, GST binds to ASK1 to lower it activity (Dorion et al. 2002). In this model ASK1 as a MAPKKK is an upstream activator of JNK cascade as a result of external signals coming from G-protein receptors. When ASK1 is released from GST and activated by an external signal it activates JNK by phosphorylation of MAPKK (Davis 2000). Most importantly, ASK1 is important to maintain JNK activation in response to ROS leading to apoptosis (Tobiume et al. 2001). Studies of ASK1 orthologs in $P$. anserina and Magnaporthe grisea reveal that inactivation of that protein leads to various effects, including crippled growth, female sterility and decreased conidia production (Kicka and Silar 2004; J.-R. Xu et al. 1998). This data may additionally indicate that conidiation during barrage formation may be activated by ASK1-related signaling.

Our transcriptome data indicates that rapid activation of detoxification mechanisms coincides with overexpression of genes producing fungal toxins and other secondary metabolites. A majority of stress genes identified in this study are known to be activated in response to high levels of ROS as a result of apoptosis (Ray et al. 2012). Importantly, we can conclude that barraging cells activate several detoxification mechanisms and activate genes that may relate to production of several types of mycotoxins. This leads us to suggest that barrage formation may involve several PCD pathways likely associated with JNK cascade. In addition, JNK-mediated PCD is activated in cells surrounding interacting hyphae. These cells get exposed to toxins 
produced in incompatible hyphae, and this could in turn activate PCD. Altogether these cells contribute to barrage formation.

\section{Vegetative incompatibility genes}

Vegetative incompatibility (vic) or heterokaryon incompatibility (het) genes determine interspecies allorecognition in many Ascomycota (Glass and Dementhon 2006; Paoletti and Saupe 2009). Well studied examples of heterokaryon incompatibility (HI) and vegetative incompatibility (VI) loci include C. parasitica, $P$. anserina and $N$. crassa (Smith and Lafontaine 2013). In each species there are 5-10 incompatibility loci and polymorphisms at these loci creates a basis for nonself recognition. Hyphal fusion of two members of the same species possessing different alleles at one or more vic loci, results in an incompatibility reaction. In $C$. parasitica VI reaction is evident as a barrage, a line of demarcation between two incompatible colonies made of dead cells, pigment deposition and/or hypertrophy. $N$. crassa, on the other hand, presents a well-studied example of HI, where barrage line is often absent. HI strains are often able to fuse and form heterokaryons while presenting common traits of incompatibility such as slow growth and high rates of cell death. As already discussed in Chapter II, one of the main genetic characteristics of vic genes is the presence of a HET domain (Smith and Lafontaine 2013). Some HET domain proteins were classified as part of the broad family of STAND proteins in the NATCH clade, showing close relation to proteins involved in innate immunity response (Leipe et al. 2004). These vic genes are mostly found in $P$. anserina genome (see Figure I-3). However, commonly in other Ascomycota, vic genes predominantly encode HET domain proteins. 
In animals, the NATCH clade is presented by groups of proteins involved in inflammatory responses and regulation of apoptosis (Harton et al. 2002). In case of incompatibility, it was hypothesised that HET domains can play a role similar to TIR domains in the AP family of STAND proteins (Paoletti and Saupe 2009). TIR domains are part of Toll-like (TLR) and NOD-like receptors, which are activators of innate immunity response, usually triggered by cellular components of foreign organisms (Fukata et al. 2009; O'Neill and Bowie 2007). As proposed by Paoletti and Saupe (2009), HET genes may present a fungal version of NLR type innate immunity. These authors proposed two possible modes of action. First, HET-domain proteins respond to the presence of a particular protein or compound originating from a foreign organism. Second, termed the "guard hypothesis", is when HET-domain proteins keep some proteins under constant surveillance and trigger downstream reaction when those proteins are modified. NLR receptors in human and fruit flies are found to activate various reactions which include the already discussed JNK apoptotic pathway and can trigger production of anti-microbial peptides and ROS (De Gregorio et al. 2002; O'Neill and Bowie 2007). Transcriptome analyses in my thesis implicate similar processes and this provides additional support for the idea that fungal incompatibility may be a type of innate immunity.

In previous section of this chapter I described possible mechanisms of PCD during barrage, which represent part three of model described at the beginning. As I also mentioned, we are moving along this model in reverse order. So now we discuss in more detail the second stage, triggers of VI. There are six known vic loci linked to VI in $C$. parasitica (Cortesi and Milgroom 1998). Recent molecular genetic analyses indicate that 
among them, the vic3, vic2 and vic4 loci do not possess genes with a HET domain. Incompatibility due to vic4 differences show a subtle, but discernable barrage, with $100 \%$ virus transmission (G. H. Choi et al. 2012). On the other hand, paired vic3 incompatible strains demonstrate a strong barrage reaction and reduced virus transmission rates (Cortesi et al. 2001). Genetic organization of the vic3 locus suggests an example of nonallelic interactions of linked genes (see Figure I-3c in Chapter I) (D. X. Zhang et al. 2014). A typical example of two tightly linked genes non-allelic interactions was shown for vic6, which is similar to het-c locus in N. crassa (G. H. Choi et al. 2012; Glass and Dementhon 2006). In the case of vic6 and het-c loci, however, one of the genes that make up the incompatibility locus contains a HET domain. Therefore, from genetic organisation of vic genes it may appear that a HET domain is not necessary component of VI reaction, at least in C. parasitica. However, other literature allows us to propose indirect involvement, when HET genes are activated by upstream regulators (Shiu and Glass 1999). This in turn also brings another proposition: that HET genes do not have to be polymorphic in two interacting strains to trigger downstream incompatibility reactions. Those propositions open a wide window for possible mechanisms of VI. As analysis of $C$. parasitica genome showed there are 124 genes annotated with a HET domain. Many of them are not differentially expressed and are not apparently associated with vegetative incompatibility, at least based on our analysis of vic3-associated incompatibility. Although an exhaustive analysis has not been carried out, most HET-containing ORFs that have been examined, do not show genetic polymorphism between strains, aside from the ones already associated with VI (G. H. Choi et al. 2012; D. X. Zhang et al. 2014). 
Data presented in Chapter II seems to support the idea that, at least in case of vic3 locus, vic genes serve as upstream regulators of several HET-domain genes. We identified seven genes that contain a HET domain of which expression is affected by vic 3 -associated barrage formation and/or the presence of the p29 viral element. Among them, six were differentially expressed in barraging strains (Figure IV-3). In this thesis, HET-domain genes that were not previously associated with any type of incompatibility, and that were not previously given genetic designations, are named as dev3, for differentially expressed during $\underline{v i c} \underline{3}$ incompatibility. These genes are sequencially designated as $\operatorname{dev} 3 a, \operatorname{dev} 3, \operatorname{dev} 3 c, \operatorname{dev} 3 g, \operatorname{dev} 3 h, \operatorname{dev} 3 i$ and $\operatorname{dev} 3 j$. Surprisingly, one HETdomain gene identified as differentially expressed during vic3 incompatibility, was vicla, which is a part of vicl incompatibility locus. Both strains paired in this experiment, P74-3 and EP155, share the same vicla allele and are compatible at the vicl locus. Gene vicla was overexpressed uniformly in all vic3-associated barraging samples. Considering this unexpected outcome, I examined RNA-seq dataset whether other known vic genes were overexpressed during vic3-incompatible interactions. As expected, none of vic2, vic4, vic6, vic7 genes were differentially expressed (Figure IV-3). So, it appears that only vic1 is coupled with vic3 VI.

The observed activation of the vicla gene and other HET domain-containing ORFs presented a possible explanation for the absence of a HET domain ORF within the $v i c 3$ locus. It is possible that vic3 genes function as upstream activators of vicla and other HET-domain genes. To examine the role of identified HET genes in other incompatibility settings, we performed a series of RT-qPCR tests (Figure IV-4). In this experiment, we 


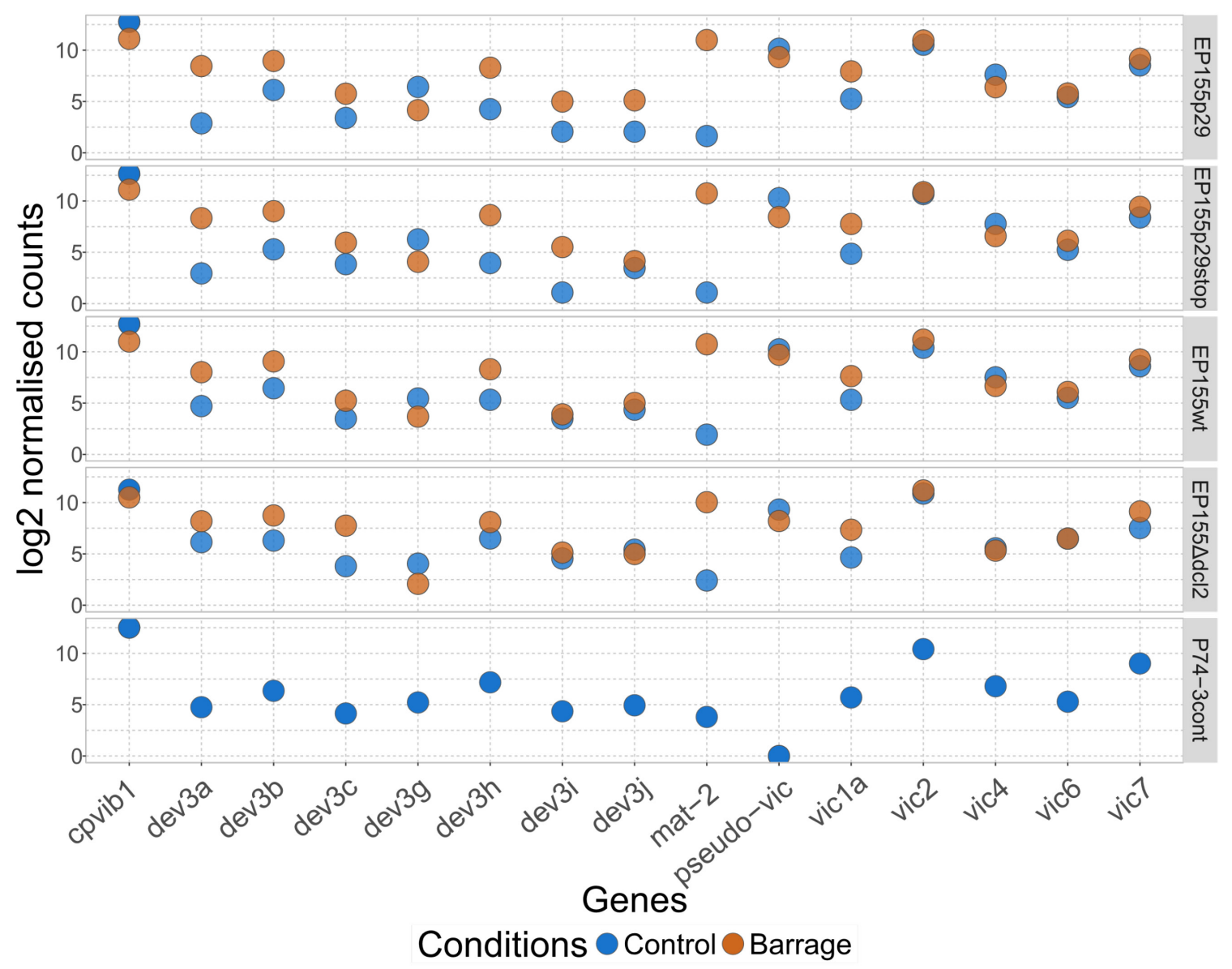

Figure IV - 3. Normalised expression rates of cpvibl, HET domain genes and vic genes during vic3 incompatibility.

Y-axis of the graph shows estimated read counts from RNA-seq analysis, normalised and transformed on a $\log 2$ scale. Gene IDs in C. parasitica genome: cpvib1 - 67224, dev3a261856, dev3b - 262887, dev3c - 268419, dev3g - 240373, vicla -258862 , dev3h238641, dev3i - 243920, dev3j-53104, mat-2 - 44005, pseudo-vic - 48444, vicla 258862, vic2 - 352811, vic4 - 255909, vic6-231803, vic7-231853. 
used a series of strain pairs, each having different mating types and a difference at one of the vic loci. To our surprise, the vicla gene appeared to be activated under all barrage settings. Although less pronounced, up-regulation is observed when the strains are incompatible for vic4, vic6 or vic7. Along with the vicla gene, dev3a showed significant overexpression in strain pairings that were incompatible by vicl, vic2, vic3 and vic4, but not for vic6- or vic7-incompatible pairings. The near universal pattern of overexpression of vicla and $\operatorname{dev} 3 a$ indicates that there is a redundancy among HET genes. Furthermore, it is plausible that some HET-domain genes could be components functioning in several incompatible reactions despite not being genetically linked to the incompatibility locus, and are also not required to be genetically polymorphic.

Among differentially expressed genes in pairings of EP155-derived strains and P74-3 we identified one polymorphic HET gene. This gene is shown in Figure IV-3 as pseudo-vic showing zero transcript accumulation in P74-3 strain with no change in expression among EP155 strains. However, it was upregulated by $\sim 4.5 \log 2$ fold in P74-3 + EP155 pairings. This pseudo-vic gene was previously studied as a possible candidate vic gene (D. X. Zhang et al. 2014). Genetic analysis showed that among four studied strains, only EU40 (Cortesi and Milgroom 1998) and EP155 contained a complete version of the whole pseudo-vic ORF. In EP155 the pseudo-vic locus contains two genes, one with a HET domain and a second with a GTFase domain. Both sequences are deleted from genomes of the other strains examined, including P74-3 (EU60). D. X. Zhang et al. (2014) concluded that this particular HET gene has lost its function as strains with differences only at this locus, do not form a barrage when confronted. Nevertheless, it is still possible that some strains in nature have a fully functional second allele of this gene. 
Genetic studies of $C$. parasitica strains throughout Europe identified six vic loci and therefore 64 possible vegetative incompatible groups (called vc types) (Milgroom and Cortesi 1999). If C. parasitica carries only these six functioning vic loci, then all newly found field samples will fall into one of these $64 \mathrm{vc}$ types. However, a study of field isolates from China and Japan identified 44 and $71 \mathrm{vc}$ types, respectively (Y.-C. Liu and Milgroom 2007). Among them only three isolates from Japan were compatible with one of the 64 European types. The rest of Asian vc types were not detected in Europe. This provides further evidence for additional vic loci beyond the six now characterised, and polymorphisms at additional HET-domain genes in C. parasitica may determine additional ve types.

How HET-domain genes are controlled at the molecular level is largely unknown aside from the well-studied case in $N$. crassa indicating at least some het incompatibility gene expression is controlled by the Ndt80p-like transcription factor vib-1 (Xiang and Glass 2002). In yeast, the p53-like protein Ndt80p is a regulator of meiosis and in $N$. crassa this transcription factor controls multiple functions including female sexual development and formation of ascospores (Hutchison and Glass 2010). Studies with $N$. crassa strains in which vib-1 is deleted indicate that strains differing at het-c are able to form viable heterokaryons (Dementhon et al. 2006). There are three paralogs of p53-like proteins in the $C$. parasitica genome and none have been previously studied in connection to incompatibility function. The ortholog with the highest similarity to vib-1 is identified as cpvib-1 (ID: 67224), showed low differential expression during vic3associated barrage (Figure IV-4). We used qPCR to examine expression levels of cpvib-1 during barrage in the previously mentioned pairings that are incompatible by single vic 


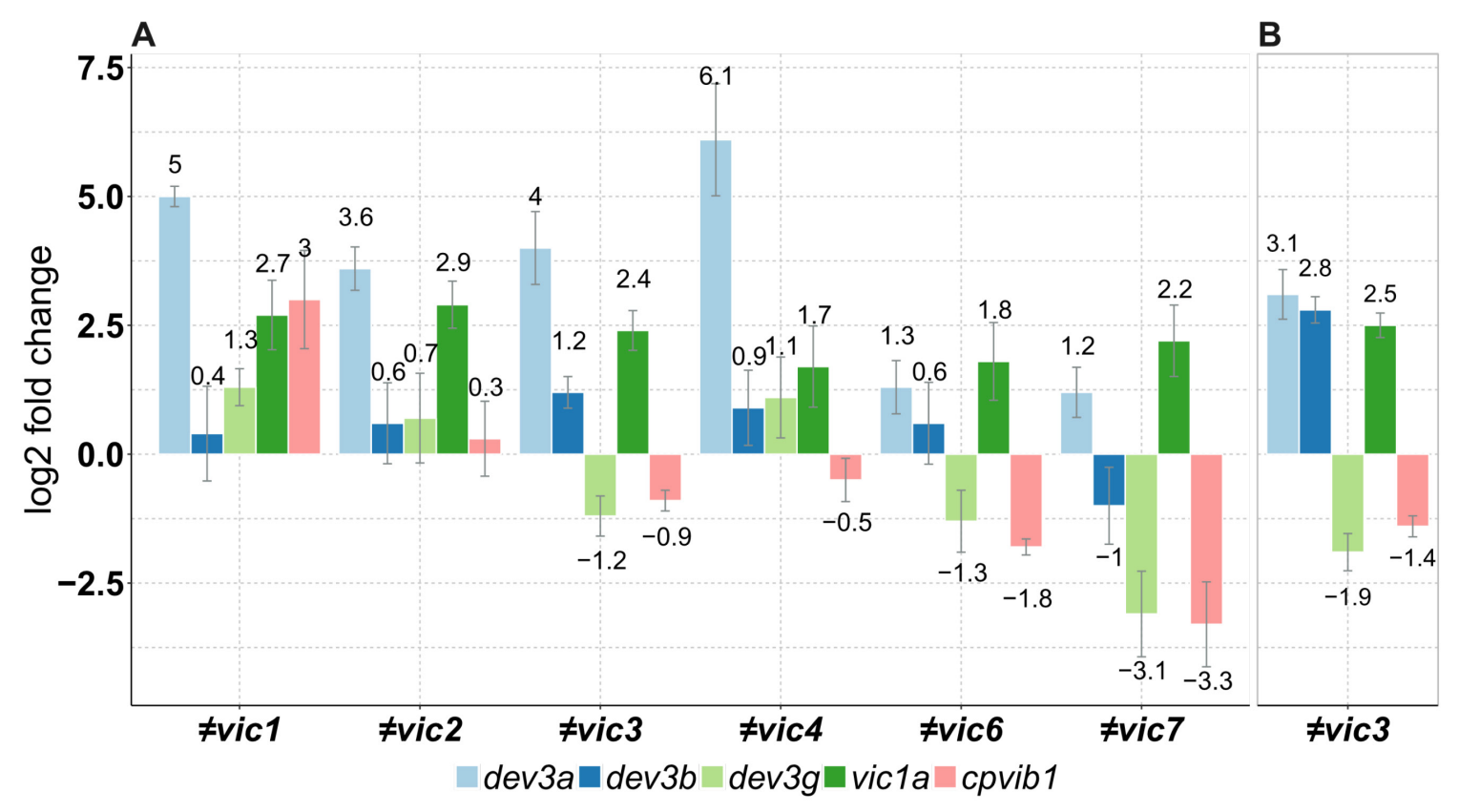

Figure IV - 4. RNA-seq and RT-qPCR data of differential expression of four HET domain genes and cpvibl gene under six types of incompatibility in $C$. parasitica.

Four HET genes used in the experiment were first identified as differentially expressed during vic3 incompatibility by RNA-seq analysis (panel B). A) RT-qPCR data of HET domain genes and cpvib-1 during barrage formation in strains incompatible by one of the vic loci. B) HET genes selected by average $\log 2$ fold differential expression $>1.5$ (cpvib1 was selected by $>1$ threshold) in vic3 incompatible strains based on RNA-seq data. Gene IDs in $C$. parasitica genome: dev3a - 261856, dev3b-262887, vicla - 258862,

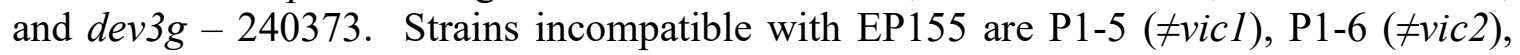

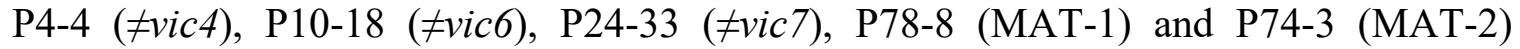
( $\neq v i c 3)$. Whiskers indicate standard error for at least four biological replicas for each gene. 
loci. According to our data, $c p v i b-1$ is not only downregulated in $\neq v i c 3$, but also in $\neq v i c 6$ and $\neq v i c 7$ pairings. In this regard, expression of the $\operatorname{dev} 3 g$ gene seems to follow the expression of cpvib-1. In vic-incompatible pairings where cpvib-1 is overexpressed or shows no change in expression compared to control, $\operatorname{dev} 3 g$ is overexpressed as well. In strain pairings incompatible by vic3, vic6 and $v i c 7, \operatorname{dev} 3 g$ is downregulated along with cpvib-1. There is no direct evidence to suggest that cpvib-1 controls HET-3g expression, and it should be noted that transcription factor function is often post-translationally regulated. Nonetheless, this may indicate that there is a regulatory relationship between HET-domain genes and p53-like proteins. On the other hand, data on Figure IV-4 indicates that not all HET-domain genes regulated by cpvib-1, implying, again, redundancy among HET genes regulators.

There is no direct connection on molecular level between HET-domain genes and PCD. In the previous section I attributed activation PCD through JNK pathway as a result of failure of detoxification mechanisms. In that regard the plausible link of HET and PCD could be through activation of toxin genes. The production of toxic secondary metabolites presents a simple explanation of HI-associated PCD mechanism in fungi. Many fungal genes associated with production of toxins are silent under normal growth conditions. However, when they are triggered, these toxins do kill not only the interacting organism, but can also kill the fungal cell itself. This simple model of PCD by intoxication during allorecognition sheds light on problems with identification of fungal PCD mechanisms. Toxins trigger extrinsic pathways along with other abiotic factors and these pathways can differ depending on the chemical nature of the activated receptor (S. Gao and Nuss 1996; Gollasch et al. 1993). Factors like ROS, growth hormone or conserved bacterial proteins 
(like flagellin), activate the JNK1/p38 death pathway through different types of G-protein coupled receptors (Ichijo 1999; O'Neill and Bowie 2007; Ray et al. 2012; Yin et al. 2000). Importantly, toxins produced internally can trigger an "extrinsic" PCD pathway, since fungi are not always able to protect themselves from their own toxins. These toxins may be pumped out from producing cells by detoxification mechanisms and their accumulation in an outside environment in turn can trigger PCD in surrounding cells.

\section{Remarks on Hyphal fusion}

Considering the interactions described above, a hyphal fusion-related pathway does not appear to play a direct role in HI associated PCD. However, many pathways share the same protein kinase activators along the way, thus fusion signaling can be one of the contributors to PCD unset. Anastomosis, or hyphal fusion, is known to occur in many Ascomycota and is well studied in N. crassa (Fleißner et al. 2009a; Glass et al. 2000), a species that is closely related to C. parasitica. In N. crassa, the oscillatory nature of anastamosis requires formation of a mitogen activated protein kinase (PKs) complex, which includes NRC-1/MEK-2/ MAK-2/HAM-5 (Fleißner et al. 2009a; Jonkers et al. 2014). These proteins form puncta complex in response to external chemical signals (Jonkers et al. 2016). This complex is homologous to yeast's pheromone response pathway (Bardwell 2004). Upstream activation of fusion MAPK cascade is associated with Ste20 (PAK) PKs and STE-50, which form a complex with NRC-1, which in turn is activated by extracellular signal from G-protein coupled receptors (Dettmann et al. 2014). Although, the nature of G-protein coupled receptors and what activates them remains unknown. As the fusion complex is well studied in N. crassa, I identified and studied some orthologs of puncta genes in C. parasitica (Figure IV-5). The gene cpnrc-1, 
ortholog of yeast Stel1 and N. crassa nrc-1, was never properly studied in C. parasitica. Similarly,

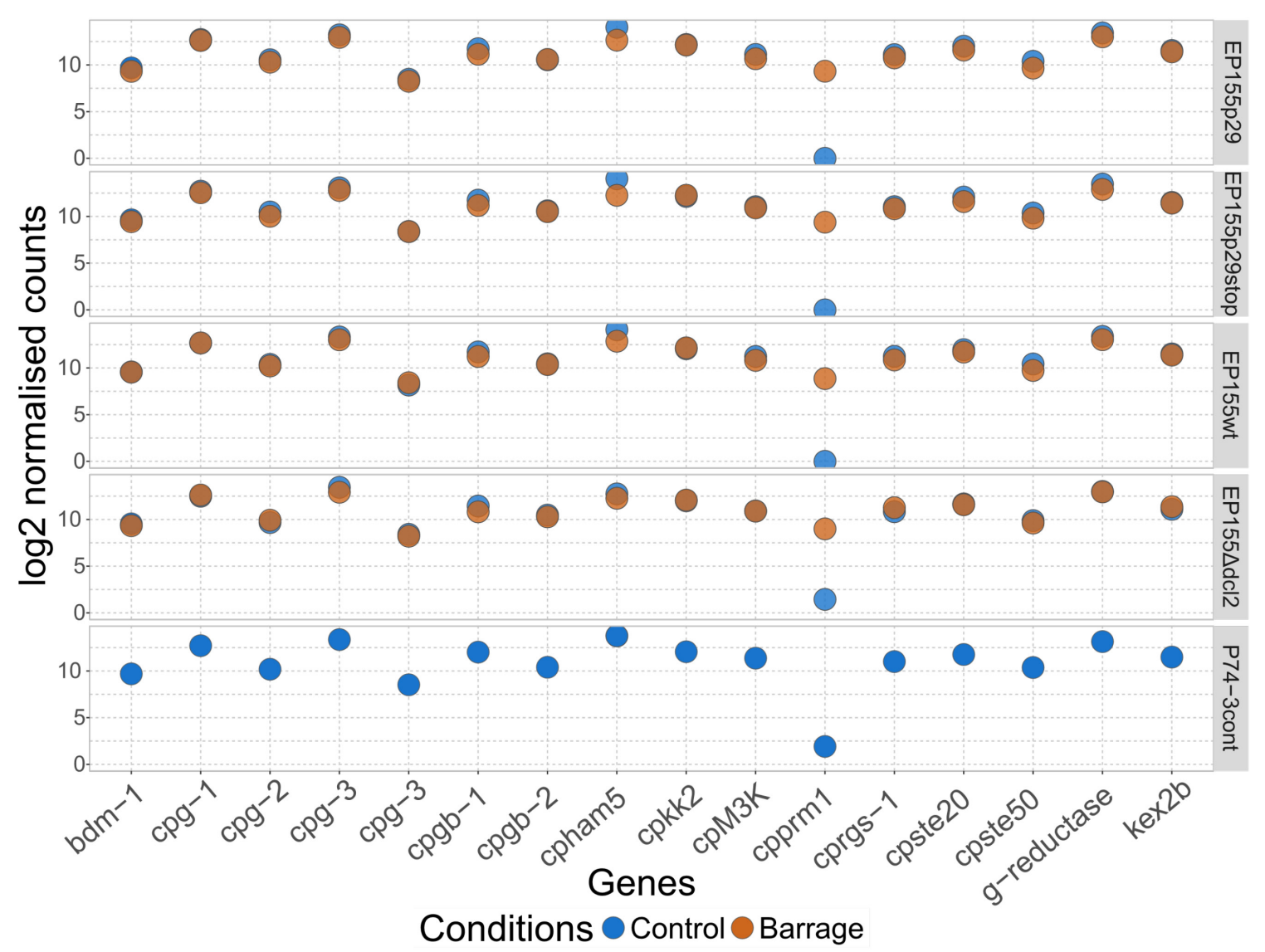

Figure IV - 5. Expression of gene orthologs associated with hyphal fusion and G-protein signaling in C. parasitica.

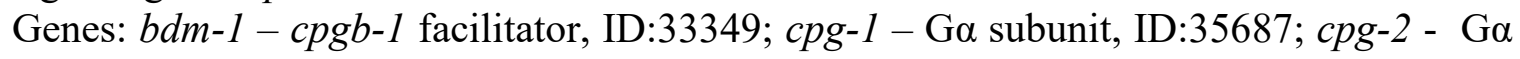
subunit, ID:89898; cpg-3 - G $\alpha$ subunit, ID:103377; $c p g b-1$ - G $\beta$ subunit, ID:63781; $c p k k 2$ - MEK-2 protein kinase, ID:103555; cpM3K - ortholog of MAP kinase, ID:66950; cprgs-1 - G-protein regulator, ID:61450; g-reductase - G-protein reductase, ID:105373; cpham-5 - scaffold protein in fusion MAK-2 pathway, homolog of yeast Ste11, novel transcript ID: NOV_007019; cpprm1 - membrane stabilising protein, yeast ortholog, ID:227067; cpste20 - PAK-like MAP kinase, ID:99781; cpste50 - regulatory subunit of NRC-1 and cpham5, ID:33424. 
the C. parasitica ortholog of $N$. crassa ham-5 was not previously annotated and characterised, although a close homolog was identified among novel transcripts (ID: 277186). The MEK-2 ortholog, cpkk2, was studied in its relation to CHV1, which revealed the deletion of $c p k k 2$ disrupts virus transmission through anastomosis (Turina et al. 2016). The authors suggest that CHV1 by affecting MAPK signalling stops the fungus development at a juvenile stage. Which prioritises vegetative growth and asexual sporulation over sexual reproduction and fruit-body formation. The direct ortholog of MAK-2, cpMK2 (ID: 103555), was also found to promote development, sporulation and sexual reproduction (E. S. Choi et al. 2005). Although, cpmk2 knockout caused a similar effect to cpkk2 knockout, the presence of CHV1 does influence the cpMK2 phosphorylation levels. On the same cascade level, there is a cpMK1, ortholog of animal p38 (S. M. Park et al. 2004). This PK promotes sporulation and pigmentation, but at the same time, inhibits pheromone gene expression. Compared to cpMK2, cpMK1 is influenced by CHV1 infection. cpMK1 is not a part of fusion MAPK cascade, but it is related to p38 and therefore may be a target of JNK/ASK1 PCD pathway (Davis 2000; Tobiume et al. 2001). This suggests that PCD activated as a result of incompatibility, may activate sporulation through PK like cpMK1.

Orthologs of NRC-1 upstream regulation of fusion were identified in $C$. parasitica genome and our transcriptome dataset. Activators in N. crassa fusion, STE-50 (ID: 33424) and STE-20 (ID: 99781) were not previously studied in C. parasitica. Gprotein signaling, on the other hand, was extensively studied in relation to CHV1 infection. These studies showed that disruption of Ga subunit gene cpg-1 (ID: 35687) causes symptoms similar to a CHV1 infection such as reduced pigmentation, lack of 
sporulation and hypovirulence (S. Gao and Nuss 1996). Disruption of other G $\alpha$ subunit genes, cpg-2 (ID: 89898) and cpg-3 (ID: 103377), did not cause significant changes in phenotype (S. Gao and Nuss 1996; Todd B. Parsley et al. 2003). Considering this, it is possible to suggest that $\mathrm{cpg}-1$ is most likely candidate for fusion receptor coupled protein, as its disruption causes similar symptoms to deactivation of PKs down the pathway (E. S. Choi et al. 2005).

From the perspective of transcription profiling and gene expression analyses, molecular processes associated with hyphal fusion described above, are almost completely in the dark, as most of the regulation here happens at the protein level. Commonly, transcription factors and PKs do not change their expression rates if processes they are involved in are activated. As seen in Figure IV-5, all gene orthologs of fusion PKs and G-protein subunits, show pretty high expression rates but these rates do not change under barrage conditions or when p29 is or is not present in EP155. From a practical perspective, the stability of their transcript levels may make them useful as reference sequences for qPCR analysis.

The only transcriptionally regulated gene involved in hyphal fusion that we were able to detect in our analysis, is an ortholog of yeast Prm1 gene (ID: 227067, cpprm1). This gene belongs to a group of pheromone regulated proteins in yeast where it is was found to be activated during fusion as a part of mating. Consequently, deletion of that gene leads to failed mating in a majority of yeast cells (Engel et al. 2010; Heiman and Walter 2000). Whereas deletion of prml in yeast causes complete loss of fusion required for mating, studies on the $N$. crassa ortholog showed that this gene deletion causes $\sim 50 \%$ decrease in mating and in vegetative fusion (Fleißner et al. 2009b). As seen in Figure IV- 
5, expression of the prm 1 ortholog in control (monoculture) C. parasitica strains is almost zero, but in barraging samples this gene is highly overexpressed. It is not clear what regulates cpprml expression or what role this gene plays in vegetative incompatibility.

\section{Conclusion}

In the beginning of previous section I divided the main VI processes into four phases: 1) hyphal fusion; 2) incompatibility trigger; 3) PCD pathway; 4) final stages of cell death. Here I will present a general overview on mechanisms of barrage formation based on analyses in this chapter. A summary diagram of these analyses is shown in Figure IV-6 where data on differential expression during barrage formation in C. parasitica, is placed in a framework of molecular interactions and pathways known for other fungi.

MAPK kinases cascade plays a central role in all main signal transduction pathways, including fusion and stress reactions (Li et al. 2005; Pandey et al. 2004; Tobiume et al. 2001). On Figure IV-6, MAPK cascade associated with hyphal fusion is activated through an unknown G-protein coupled receptor by chemical signaling (Jonkers et al. 2016). It then transmits the signal to MAP kinases associated with puncta formation. This PK complex is homologous to the yeast transduction pathway activated by mating pheromones (Jonkers et al. 2014; Maeder et al. 2007). Similarly to yeast, this pathway leads to activation of Ste12 orthologs in N. crassa and C. parasitica (Leeder et al. 2013; Maeder et al. 2007). 


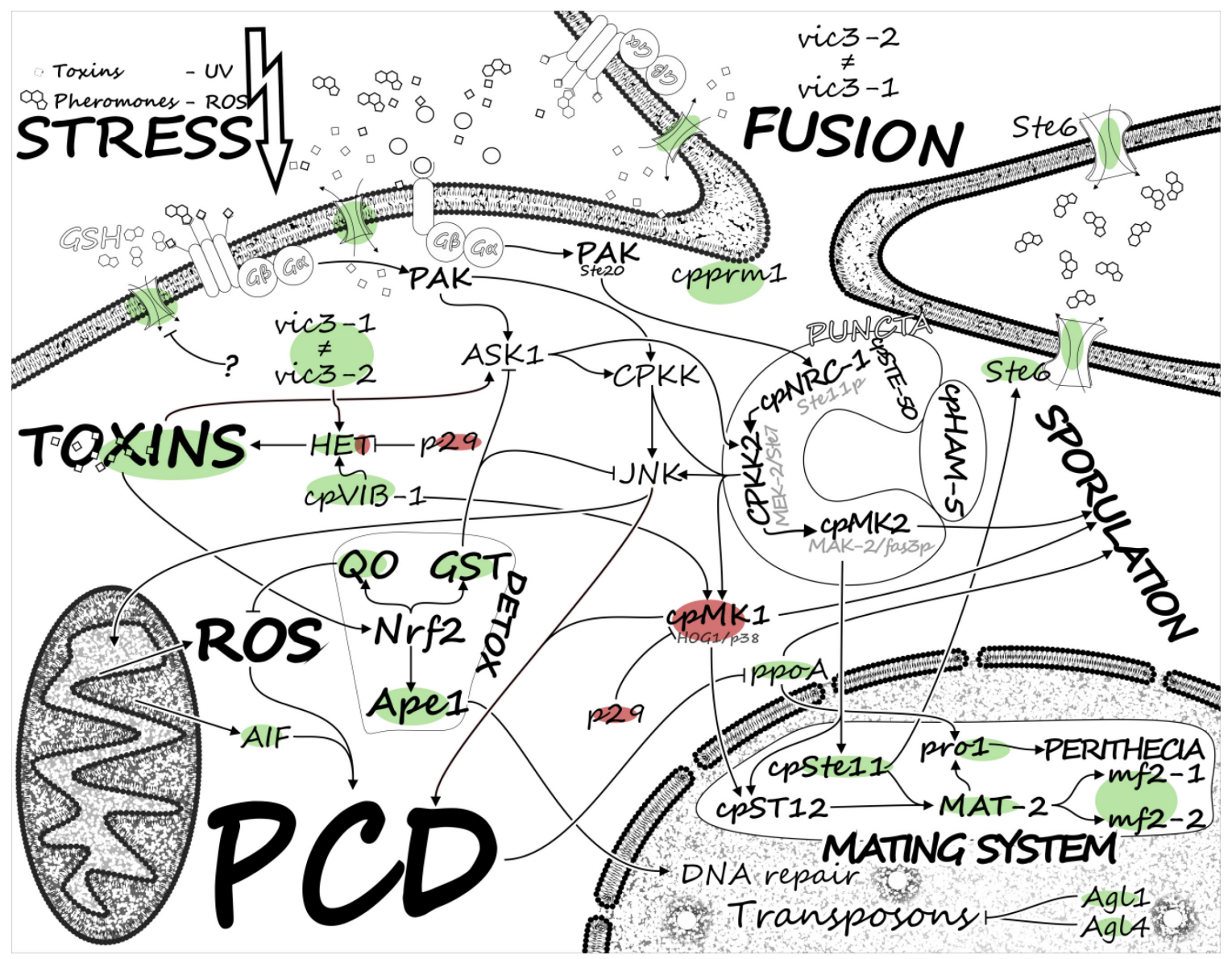

Figure IV - 6. Molecular pathways during barrage formation in C. parasitica vic3 incompatible strains.

Green highlight indicates transcriptionally regulated components of the pathways identified in this study. Red highlight indicates components of the pathways influenced by $\mathrm{p} 29$. In puncta complex, grey text under protein name indicates names of protein orthologs in yeast or $N$. crassa. For more details On green and red highlighted terms see: p.117 - GSH transporter; p.50 - Toxins; p.122 - HET genes, affected by p29; p.128 cpVIB-1; p.115 - Detox genes; p.119 - AIF and ROS; p.112 - ste6 and cpste11; p.106 pheromone genes mf2-1 and mf2-2 and MAT; p.58 - Argonaut genes agl1 and ag14, possibly involved in transposons silencing; p133 - cpMK1 protein kinase influenced by p29. 
At that point in fused incompatible hyphae HET-domain proteins trigger PCD. Signaling for activation of JNK/ASK PCD pathway may go through similar MAPK cascade as fusion. On the figure, G-protein coupled receptor reacts to externally presented toxins and activates the pathway. This presents the example of externally activated PCD, as a result of high toxicity. This pathway can also be activated in cells surrounding interacting hyphae. Here stress signals in the ASK1/JNK pathway can employ same PKs as in fusion pathway. As both fusion and stress activate similar PKs cascade, and both processes happen simultaneously, it is hard to deduce which one is responsible for activation of downstream processes.

We can also suggest that similar JNK/ASK pathway may be activated from inside the cell by internally produced toxins. It is not known for certain how HET-domain proteins function, but we can suggest they can be externally or internally activated by signaling molecules. This proposition comes from observations that Toll-like receptors (TLR) can present themselves outside of the cell membrane or detect the signal by being in the cytoplasm, but still activate similar pathways downstream (Leulier and Lemaitre 2008). Additionally, if HET-domain proteins function similarly to NLR, it is possible that they also activate cell defence mechanisms. In case of hyphal fusion defence reaction can start with mycotoxins production and eventually PCD as studies showed internally produced toxins may be harmful to the producing cells themselves (Owens et al. 2015).

Stress and, in some cases fusion, further activate asexual sporulation with mating pheromones being an essential component of conidia (Adams et al. 1998; H.-S. Park and $\mathrm{Yu}$ 2012). This process most likely happens through cpMK2 and possibly cpMK1. Studies indicated that cpMK2 is not influenced by CHV1 but cpMK1 is (H.-S. Park and 
Yu 2012). Additionally, as discussed in Chapter I (see Figure I-6), one of the main symptoms of CHV1 infection is a lack of conidiation. At the same time observations show that infected incompatible strains still form a barrage with heavy conidiation. From that we can suggest that activation of asexual sporulation in monocultures happens through cpMK1. However, during barrage formation sporulation is activated through a pathway fusion that involves cpMK2. Considering that cpMK2 is not influenced by p29 or CHV1, allows barraging hyphae to activate conidia formation. If we consider that cpMK2 is active only during fusion, we can suggest that conidiation in monocultures can be governed exclusively by cpMK1 pathway. This creates a situation where infected strains fail to produce conidia, but barraging hyphae heavily conidiate.

Activation of allorecognition and programmed cell death presumably happens right after hyphal fusion takes place. We assume that, in the case of vic3 incompatibility in $C$. parasitica, vic $3 a-1$ and vic $3 b-2$ proteins from opposing strains interact, along with interaction of vic $3 b-1$ and vic $3 a-2$, and this triggers a PCD response. Similar mechanisms were proposed in models for het-c/pin-c in N. crassa and vic6-pix6 in C. parasitica (G. H. Choi et al. 2012; Glass and Dementhon 2006). In the later of these models, the small protein, PIX6 are inferred to quickly move into the opposing cytoplasm to trigger incompatibility by interacting with VIC6. On the other hand, we cannot ignore the possibility that genes activated by vic3 are associated with transmembrane receptors, following the analogy with TLR receptors (Leulier and Lemaitre 2008; O'Neill and Bowie 2007). A closer association for vic3 system may be seen in mat incompatibility in N. crassa (Shiu and Glass 1999; Xiang and Glass 2004). Here, tol is a HET-domain gene that is operational during vegetative growth and modulates PCD when fused strains have 
opposite mating types. Strains with inactivated tol are able to form viable heterokaryon involving nuclei with different mating types. The difference with mat and vic3 incompatibility is that vic3 appears to activate several non-polymorphic HET-domain genes and thus may present an example of a highly redundant system.

Going down the pathway, at the end of MAPK cascade, we find the already mentioned cpMK2 (ortholog of $N$. crassa MAK-2 and yeast Fas3p) and cpMK1. Following the analogy with yeast and $N$. crassa models, cpMK1 and cpMK2 most likely trigger the $p p-1 /$ Ste12 ortholog cpST12. As in yeast cpST12 is transcription factor, most likely involved in regulation of mating processes ( $\mathrm{Li}$ et al. 2005). In this case, the most likely target of cpST12 is the mating-type locus (MAT-1 or MAT-2), which in turn activates subsequent reactions, such as pheromone genes and perithecia formation (Figure IV-6). In RNA-seq analysis presented in Chapter II, we analysed expression in strains EP155 and P74-3, which both belong to mating type MAT-2. The mating-type locus in $C$. parasitica shows similar idiomorphic nature of organisation as many other Pyrenomycetes (McGuire et al. 2001). The MAT-1 locus consists of three genes while MAT-2 carries only one. Our data indicated that MAT-2 was among the highly differentially expressed genes in vic3-incompatible interactions. In addition, we monitored MAT-2 expression using qPCR from $2^{\text {nd }}$ to the $3^{\text {rd }}$ day after inoculation in monocultures and barraging strains. Expression of MAT-2 on the $3^{\text {rd }}$ day appears similar to what we see in RNA-seq data (Table S1) in barraging strains. If pheromone genes in monoculture within three days after inoculation remain silent, the MAT-2 transcript is already detected on the second day of growth and its expression gradually increases with 
age (data not shown). In that sense, MAT genes may appear useful as early indicators of sporulation and vegetative incompatibility.

MAT-2 is a transcription factor, which activates mating process. Due to MAT-2 activation and data in the literature we can deduce that MAT genes activate pheromones in response to external signals (Kües and Casselton 1992; Marra and Milgroom 2001). Perithecia formation in $C$. parasitica is associated with activation of transcription factor pro1 (Masloff et al. 2002; Sun et al. 2009b). It is not known what transcriptional regulator activates prol, but we can suggest that the most likely candidate is MAT. However, the function of prol downstream is probably associated with $p p o A$ ortholog. In Aspergillus expression of ppoA, encoding a fatty acid dioxygenase, is associated with regulation of anamorph and teleomorph phases. Overexpression of ppoA causes a developmental shift, which increases ratio of sexual spores in Aspergillus (Tsitsigiannis et al. 2004). In relation to barrage formation in $C$. parasitica we observed partial transcriptional activation of genes involved in sexual reproduction. MAT genes as a main activator, followed by pheromones, which become a part of conidia. At the same time, we see deactivation of downstream mating genes like ppo $A$ and prol, and lack of any sexual structure development. Here we suggest that mating system activation is important to supply forming conidia with mating pheromones. The rest of system, which involves fruit-body formation is not activated.

Here, at the end of the chapter, I'll summarise the data discussed above and shown on Figure IV-6. A most probable result of HET gene activation is of the initiation of mycotoxins biosynthesis, as we observe in our dataset. Stress caused by toxins coming from intra- and extracellular environment activate PCD associated ASK1/JNK1 pathways 
(Tobiume et al. 2001; Yin et al. 2000). JNK in turn helps to release ROS and AIF from mitochondria and subsequently activate PCD. Upstream activation of this pathway happens through external receptors, like TLR in animal models (Moresco et al. 2011) or just stress factors like toxins or ROS (Fujino et al. 2006).

Increased accumulation of toxins and ROS would likely activate detoxification mechanisms. In our analysis, we were able to detect components transcriptionally activated by Nrf2 transcription factor in response to ROS and toxins (Johnson et al. 2008). The most notable gene controlled by Nrf2, glutathione-S-transferase (GST), was highly overexpressed during barrage. Most of relevant studies were done on animal models, where main function of GST is to deactivate and help to remove toxins from the cell, but in certain cases it contributes to normalisation of redox balance (Tew and Townsend 2012). Another important feature of GST is that it inhibits activation of JNK and ASK1 kinases, and in this way slows the onset of PCD (Adler et al. 1999; Ray et al. 2012). In redox balance case, quinine oxidoreductase $(\mathrm{QO})$ plays a main role in deactivation of quinones and its ROS derivatives (Jaiswal 2000). And lastly in our dataset APE1, responsible for DNA damage control, showed slight increase in expression. This data indicates that barraging cells create a highly toxic environment inside themselves and in the surrounding environment. PCD is triggered when concentrations of toxins becomes overly high and ROS are increased to the point when detoxification proteins are not able to maintain the balance. This in turn triggers asexual sporulation and the production of conidiophores. 


\section{References}

Adams, Thomas H., Wieser, Jenny K., and Yu, Jae-Hyuk (1998), 'Asexual Sporulation in Aspergillus nidulans', Microbiology and Molecular Biology Reviews, 62 (1), 3554.

Adler, V., et al. (1999), 'Regulation of JNK signaling by GSTp', EMBO J, 18 (5), 132134.

Albrecht, M., Ruther, A., and Sandmann, G. (1997), 'Purification and biochemical characterization of a hydroxyneurosporene desaturase involved in the biosynthetic pathway of the carotenoid spheroidene in Rhodobacter sphaeroides', J Bacteriol, 179 (23), 7462-7.

Allen, T. D. and Nuss, D. L. (2004), 'Specific and common alterations in host gene transcript accumulation following infection of the chestnut blight fungus by mild and severe hypoviruses', J Virol, 78 (8), 4145-55.

Allen, Todd D., Dawe, Angus L., and Nuss, Donald L. (2003), 'Use of cDNA Microarrays To Monitor Transcriptional Responses of the Chestnut Blight Fungus Cryphonectria parasitica to Infection by Virulence-Attenuating Hypoviruses', Eukaryotic Cell, 2 (6), 1253-65.

Anagnostakis, Sandra L. (1982), 'Biological Control of Chestnut Blight', Science, 215 (4532), 466-71.

--- (2001), 'American chestnut sprout survival with biological control of the chestnutblight fungus population', Forest Ecology and Management, 152 (1), 225-33.

Bardwell, Lee (2004), 'A walk-through of the yeast mating pheromone response pathway', Peptides, 25 (9), 1465-76.

Barhoom, Sima and Sharon, Amir (2007), 'Bcl-2 proteins link programmed cell death with growth and morphogenetic adaptations in the fungal plant pathogen Colletotrichum gloeosporioides', Fungal Genetics and Biology, 44 (1), 32-43.

Bechinger, Clemens, et al. (1999), 'Optical Measurements of Invasive Forces Exerted by Appressoria of a Plant Pathogenic Fungus', Science, 285 (5435), 1896-99.

Bertram, Gwyneth, et al. (1996), 'Structure and regulation of the Candida albicans ADH1 gene encoding an immunogenic alcohol dehydrogenase', Yeast, 12 (2), 115-27.

Bhattacharyya, Roby P., et al. (2006), 'The Ste5 Scaffold Allosterically Modulates Signaling Output of the Yeast Mating Pathway', Science, 311 (5762), 822-26.

Bidard, Frédérique, Clavé, Corinne, and Saupe, Sven J. (2013), 'The Transcriptional Response to Non-Self in the Fungus Podospora anserina', G3: Genes $\mid$ Genomes $\mid$ Genetics.

Biella, S., et al. (2002), 'Programmed cell death correlates with virus transmission in a filamentous fungus', Proc Biol Sci, 269 (1506), 2269-76.

Bistis, G. N. (1981), 'Chemotropic Interactions between Trichogynes and Conidia of Opposite Mating-Type in Neurospora crassa', Mycologia, 73 (5), 959-75.

Brewer, Lawrence G. (1995), 'Ecology of Survival and Recovery from Blight in American Chestnut Trees (Castanea dentata (Marsh.) Borkh.) in Michigan', Bulletin of the Torrey Botanical Club, 122 (1), 40-57.

Briza, P., Eckerstorfer, M., and Breitenbach, M. (1994), 'The sporulation-specific enzymes encoded by the DIT1 and DIT2 genes catalyze a two-step reaction 
leading to a soluble LL-dityrosine-containing precursor of the yeast spore wall', Proc Natl Acad Sci U S A, 91 (10), 4524-8.

Briza, P., et al. (1990), 'Isolation of two developmentally regulated genes involved in spore wall maturation in Saccharomyces cerevisiae', Genes Dev, 4 (10), 1775-89.

Brown, Jeffrey L., et al. (1996), 'Human Ste20 homologue hPAK1 links GTPases to the JNK MAP kinase pathway', Current Biology, 6 (5), 598-605.

Burow, G. B., et al. (1997), 'Seed Lipoxygenase Products Modulate Aspergillus

Mycotoxin Biosynthesis', Molecular Plant-Microbe Interactions, 10 (3), 380-87.

Carmona-Gutierrez, D., et al. (2010), 'Apoptosis in yeast: triggers, pathways, subroutines', Cell Death Differ, 17 (5), 763-73.

Caten, C. E. (1972), 'Vegetative incompatibility and cytoplasmic infection in fungi', $J$ Gen Microbiol, 72 (2), 221-9.

Cazanave, S., et al. (2007), 'High hepatic glutathione stores alleviate Fas-induced apoptosis in mice', J Hepatol, 46 (5), 858-68.

Chen, Baoshan, Geletka, Lynn M., and Nuss, Donald L. (2000), 'Using Chimeric Hypoviruses To Fine-Tune the Interaction between a Pathogenic Fungus and Its Plant Host', Journal of Virology, 74 (16), 7562-67.

Chen, Jinqiang, et al. (1995), 'Regulation of Cytochrome P450 2C11 (CYP2C11) Gene Expression by Interleukin-1, Sphingomyelin Hydrolysis, and Ceramides in Rat Hepatocytes', Journal of Biological Chemistry, 270 (42), 25233-38.

Choi, E. S., et al. (2005), 'Characterization of the ERK homologue CpMK2 from the chestnut blight fungus Cryphonectria parasitica', Microbiology, 151 (Pt 5), 134958.

Choi, G. H. and Nuss, D. L. (1992), 'A viral gene confers hypovirulence-associated traits to the chestnut blight fungus', The EMBO Journal, 11 (2), 473-77.

Choi, G. H., Shapira, R., and Nuss, D. L. (1991), 'Cotranslational autoproteolysis involved in gene expression from a double-stranded RNA genetic element associated with hypovirulence of the chestnut blight fungus', Proc Natl Acad Sci $U S A, 88$ (4), 1167-71.

Choi, G. H., Larson, T. G., and Nuss, D. L. (1992), 'Molecular analysis of the laccase gene from the chestnut blight fungus and selective suppression of its expression in an isogenic hypovirulent strain', Mol Plant Microbe Interact, 5 (2), 119-28.

Choi, G. H., et al. (1993), 'Molecular analysis and overexpression of the gene encoding endothiapepsin, an aspartic protease from Cryphonectria parasitica', Gene, 125 (2), 135-41.

Choi, G. H., et al. (2012), 'Molecular Characterization of Vegetative Incompatibility Genes That Restrict Hypovirus Transmission in the Chestnut Blight Fungus Cryphonectria parasitica', Genetics, 190 (1), 113-27.

Chu, Shelley and Herskowitz, Ira (1998), 'Gametogenesis in Yeast Is Regulated by a Transcriptional Cascade Dependent on Ndt80', Molecular Cell, 1 (5), 685-96.

Churchill, A. C. L., et al. (1990), 'Transformation of the fungal pathogen Cryphonectria parasitica with a variety of heterologous plasmids', Current Genetics, 17 (1), 2531.

Circu, M. L. and Aw, T. Y. (2012), 'Glutathione and modulation of cell apoptosis', Biochim Biophys Acta, 1823 (10), 1767-77. 
Cook, J G, et al. (1996), 'Two novel targets of the MAP kinase Kss1 are negative regulators of invasive growth in the yeast Saccharomyces cerevisiae', Genes \& Development, 10 (22), 2831-48.

Coroneos, Emmanuel, et al. (1995), 'Differential Regulation of Sphingomyelinase and Ceramidase Activities by Growth Factors and Cytokines: IMPLICATIONS FOR CELLULAR PROLIFERATION AND DIFFERENTIATION', Journal of Biological Chemistry, 270 (40), 23305-09.

Cortesi, P. and Milgroom, M. G. (1998), 'Genetics of vegetative incompatibility in cryphonectria parasitica', Appl Environ Microbiol, 64 (8), 2988-94.

Cortesi, P., et al. (2001), 'Genetic control of horizontal virus transmission in the chestnut blight fungus, Cryphonectria parasitica', Genetics, 159 (1), 107-18.

Craven, M G, et al. (1993), 'Papain-like protease p29 as a symptom determinant encoded by a hypovirulence-associated virus of the chestnut blight fungus', Journal of Virology, 67 (11), 6513-21.

D L Eaton, and and Gallagher, E P (1994), 'Mechanisms of Aflatoxin Carcinogenesis', Annual Review of Pharmacology and Toxicology, 34 (1), 135-72.

Daboussi, Marie-Josée and Capy, Pierre (2003), 'Transposable Elements in Filamentous Fungi', Annual Review of Microbiology, 57 (1), 275-99.

Dan, Ippeita, Watanabe, Norinobu M, and Kusumi, Akihiro (2001), 'The Ste20 group kinases as regulators of MAP kinase cascades', Trends in cell biology, 11 (5), 22030 .

Davis, Roger J. (2000), 'Signal Transduction by the JNK Group of MAP Kinases', Cell, 103 (2), 239-52.

Dawe, Angus L. and Nuss, Donald L. (2001), 'Hypoviruses and Chestnut Blight: Exploiting Viruses to Understand and Modulate Fungal Pathogenesis', Annual Review of Genetics, 35 (1), 1-29.

Dawe, Angus L., et al. (2003), 'An ordered collection of expressed sequences from Cryphonectria parasitica and evidence of genomic microsynteny with Neurosporacrassa and Magnaporthegrisea', Microbiology, 149 (9), 2373-84.

De Gregorio, Ennio, et al. (2002), 'The Toll and Imd pathways are the major regulators of the immune response in Drosophila', The EMBO Journal, 21 (11), 2568-79.

Debets, Alfons J. M. and Griffiths, Anthony J. F. (1998), 'Polymorphism of het-genes prevents resource plundering in Neurospora crassa', Mycological Research, 102 (11), 1343-49.

Degterev, Alexei, Boyce, Michael, and Yuan, Junying (2003), 'A decade of caspases', Oncogene, 22 (53), 8543-67.

Dementhon, Karine, Iyer, Gopal, and Glass, N. Louise (2006), 'VIB-1 Is Required for Expression of Genes Necessary for Programmed Cell Death in Neurospora crassa', Eukaryotic Cell, 5 (12), 2161-73.

Deng, F. and Nuss, D. L. (2008), 'Hypovirus papain-like protease p48 is required for initiation but not for maintenance of virus RNA propagation in the chestnut blight fungus Cryphonectria parasitica', J Virol, 82 (13), 6369-78.

Deng, F., Allen, T. D., and Nuss, D. L. (2007), 'Ste12 transcription factor homologue CpST12 is down-regulated by hypovirus infection and required for virulence and female fertility of the chestnut blight fungus Cryphonectria parasitica', Eukaryot Cell, 6 (2), 235-44. 
Dettmann, A., et al. (2014), 'Fungal communication requires the MAK-2 pathway elements STE-20 and RAS-2, the NRC-1 adapter STE-50 and the MAP kinase scaffold HAM-5', PLoS Genet, 10 (11), e1004762.

Di Stasi, Delia, et al. (2005), 'DHCR24 gene expression is upregulated in melanoma metastases and associated to resistance to oxidative stress-induced apoptosis', International Journal of Cancer, 115 (2), 224-30.

Dillon, D and Stadler, D (1994), 'Spontaneous mutation at the mtr locus in neurospora: the molecular spectrum in wild-type and a mutator strain', Genetics, 138 (1), 6174.

Dohlman, H. G. and Thorner, J. W. (2001), 'Regulation of G protein-initiated signal transduction in yeast: paradigms and principles', Annu Rev Biochem, 70, 703-54.

Dorion, Sonia, Lambert, Herman, and Landry, Jacques (2002), 'Activation of the p38 Signaling Pathway by Heat Shock Involves the Dissociation of Glutathione STransferase Mu from Ask1', Journal of Biological Chemistry, 277 (34), 30792-97.

Eaton, D L and Bammler, T K (1999), 'Concise review of the glutathione S-transferases and their significance to toxicology', Toxicological Sciences, 49 (2), 156-64.

Engel, Alex, Aguilar, Pablo S., and Walter, Peter (2010), 'The Yeast Cell Fusion Protein Prm1p Requires Covalent Dimerization to Promote Membrane Fusion', PLOS ONE, 5 (5), e10593.

Essigmann, J. M., et al. (1977), 'Structural identification of the major DNA adduct formed by aflatoxin B1 in vitro', Proc Natl Acad Sci US A, 74 (5), 1870-4.

Fedorova, N. D., et al. (2005), 'Comparative analysis of programmed cell death pathways in filamentous fungi', BMC Genomics, 6, 177.

Fischer, Wolf-Nicolas, et al. (1998), 'Amino acid transport in plants', Trends in Plant Science, 3 (5), 188-95.

Fleißner, A., Simonin, Anna R., and Glass, N. Louise (2008), 'Cell Fusion in the Filamentous Fungus, Neurospora crassa', in Elizabeth H. Chen (ed.), Cell Fusion: Overviews and Methods (Totowa, NJ: Humana Press), 21-38.

Fleißner, A., et al. (2009a), 'Oscillatory recruitment of signaling proteins to cell tips promotes coordinated behavior during cell fusion', Proc Natl Acad Sci U S A, 106 (46), 19387-92.

Fleißner, André and Herzog, Stephanie (2016), 'Signal exchange and integration during self-fusion in filamentous fungi', Seminars in Cell \& Developmental Biology, 57, 76-83.

Fleißner, André, Diamond, Spencer, and Glass, N. Louise (2009b), 'The Saccharomyces cerevisiae PRMI Homolog in Neurospora crassa Is Involved in Vegetative and Sexual Cell Fusion Events but Also Has Postfertilization Functions', Genetics, $181(2), 497-510$.

Fleißner, André, et al. (2005), 'The so Locus Is Required for Vegetative Cell Fusion and Postfertilization Events in Neurospora crassa', Eukaryotic Cell, 4 (5), 920-30.

Freinkel, Susan (2007), American chestnut: the life, death, and rebirth of a perfect tree (Univ of California Press).

Fuchs, Beth Burgwyn and Mylonakis, Eleftherios (2006), 'Using non-mammalian hosts to study fungal virulence and host defense', Current Opinion in Microbiology, 9 (4), 346-51. 
Fujii, I., et al. (2001), 'Identification of Claisen cyclase domain in fungal polyketide synthase WA, a naphthopyrone synthase of Aspergillus nidulans', Chem Biol, 8 (2), 189-97.

Fujino, Go, et al. (2006), 'Thioredoxin and protein kinases in redox signaling', Seminars in Cancer Biology, 16 (6), 427-35.

Fukata, Masayuki, Vamadevan, Arunan S., and Abreu, Maria T. (2009), 'Toll-like receptors (TLRs) and Nod-like receptors (NLRs) in inflammatory disorders', Seminars in Immunology, 21 (4), 242-53.

Fukuzawa, N., et al. (2010), 'HC-Pro, a potyvirus RNA silencing suppressor, cancels cycling of Cucumber mosaic virus in Nicotiana benthamiana plants', Virus Genes, 40 (3), 440-6.

Gao, Kun, et al. (2013), 'CpBir1 is required for conidiation, virulence and anti-apoptotic effects and influences hypovirus transmission in Cryphonectria parasitica', Fungal Genetics and Biology, 51 (0), 60-71.

Gao, S. and Nuss, D. L. (1996), 'Distinct roles for two G protein alpha subunits in fungal virulence, morphology, and reproduction revealed by targeted gene disruption', Proc Natl Acad Sci U S A, 93 (24), 14122-7.

Girard, A., et al. (2006), 'A germline-specific class of small RNAs binds mammalian Piwi proteins', Nature, 442 (7099), 199-202.

Glass, N. L. and Kaneko, Isao (2003), 'Fatal Attraction: Nonself Recognition and Heterokaryon Incompatibility in Filamentous Fungi', Eukaryotic Cell, 2 (1), 1-8.

Glass, N. L. and Dementhon, K. (2006), 'Non-self recognition and programmed cell death in filamentous fungi', Curr Opin Microbiol, 9 (6), 553-8.

Glass, N. L., Jacobson, D. J., and Shiu, P. K. (2000), 'The genetics of hyphal fusion and vegetative incompatibility in filamentous ascomycete fungi', Annu Rev Genet, 34, 165-86.

Gollasch, M, et al. (1993), 'Gi2 and protein kinase C are required for thyrotropinreleasing hormone-induced stimulation of voltage-dependent $\mathrm{Ca} 2+$ channels in rat pituitary GH3 cells', Proceedings of the National Academy of Sciences, 90 (13), 6265-69.

Goryachev, Andrew B., et al. (2012), 'Excitable behavior can explain the "ping-pong" mode of communication between cells using the same chemoattractant', BioEssays, 34 (4), 259-66.

Grahl, Nora, et al. (2011), 'In vivo Hypoxia and a Fungal Alcohol Dehydrogenase Influence the Pathogenesis of Invasive Pulmonary Aspergillosis', PLOS Pathogens, 7 (7), e1002145.

Guaragnella, Nicoletta, et al. (2006), 'YCA1 participates in the acetic acid induced yeast programmed cell death also in a manner unrelated to its caspase-like activity', FEBS Letters, 580 (30), 6880-84.

Gustin, Michael C., et al. (1998), 'MAP Kinase Pathways in the YeastSaccharomyces cerevisiae', Microbiology and Molecular Biology Reviews, 62 (4), 1264-300.

Hamann, Andrea, Brust, Diana, and Osiewacz, Heinz D. (2007), 'Deletion of putative apoptosis factors leads to lifespan extension in the fungal ageing model Podospora anserina', Molecular Microbiology, 65 (4), 948-58.

Harris, Helen J., et al. (2010), 'Claudin Association with CD81 Defines Hepatitis C Virus Entry', Journal of Biological Chemistry, 285 (27), 21092-102. 
Harton, Jonathan A., et al. (2002), 'Cutting Edge: CATERPILLER: A Large Family of Mammalian Genes Containing CARD, Pyrin, Nucleotide-Binding, and LeucineRich Repeat Domains', The Journal of Immunology, 169 (8), 4088-93.

Hausmann, Alexander and Sandmann, Gerhard (2000), 'A Single Five-Step Desaturase Is Involved in the Carotenoid Biosynthesis Pathway to $\beta$-Carotene and Torulene in Neurospora crassa', Fungal Genetics and Biology, 30 (2), 147-53.

Heiman, M. G. and Walter, P. (2000), 'Prm1p, a pheromone-regulated multispanning membrane protein, facilitates plasma membrane fusion during yeast mating', $J$ Cell Biol, 151 (3), 719-30.

Hillman, B. I. and Suzuki, N. (2004), 'Viruses of the chestnut blight fungus, Cryphonectria parasitica', Adv Virus Res, 63, 423-72.

Houwing, S., et al. (2007), 'A role for Piwi and piRNAs in germ cell maintenance and transposon silencing in Zebrafish', Cell, 129 (1), 69-82.

Huang, D. W., Sherman, B. T., and Lempicki, R. A. (2009), 'Systematic and integrative analysis of large gene lists using DAVID bioinformatics resources', Nature Protocols, 4 (1), 44-57.

Huang da, W., Sherman, B. T., and Lempicki, R. A. (2009), 'Bioinformatics enrichment tools: paths toward the comprehensive functional analysis of large gene lists', Nucleic Acids Res, 37 (1), 1-13.

Hutchison, E. A. and Glass, N. Louise (2010), 'Meiotic Regulators Ndt80 and Ime2 Have Different Roles in Saccharomyces and Neurospora', Genetics, 185 (4), 1271-82.

Hutchison, E. A., et al. (2009), 'Transcriptional profiling and functional analysis of heterokaryon incompatibility in Neurospora crassa reveals that reactive oxygen species, but not metacaspases, are associated with programmed cell death', Microbiology, 155 (Pt 12), 3957-70.

Hutvagner, G. and Simard, M. J. (2008), 'Argonaute proteins: key players in RNA silencing', Nat Rev Mol Cell Biol, 9 (1), 22-32.

Ichijo, H. (1999), 'From receptors to stress-activated MAP kinases', Oncogene, 18 (45), 6087-93.

Ishikawa, Noriyasu, et al. (2014), 'Non-Heme Dioxygenase Catalyzes Atypical Oxidations of 6,7-Bicyclic Systems To Form the 6,6-Quinolone Core of Viridicatin-Type Fungal Alkaloids', Angewandte Chemie International Edition, 53 (47), $12880-84$.

Jacob-Wilk, D., Turina, M., and Van Alfen, N. K. (2006), 'Mycovirus cryphonectria hypovirus 1 elements cofractionate with trans-Golgi network membranes of the fungal host Cryphonectria parasitica', J Virol, 80 (13), 6588-96.

Jacobson, D. J., Beurkens, K., and Klomparens, K. L. (1998), 'Microscopic and Ultrastructural Examination of Vegetative Incompatibility in Partial Diploids Heterozygous athetLoci inNeurospora crassa', Fungal Genetics and Biology, 23 (1), 45-56.

Jaiswal, A. K. (2000), 'Regulation of genes encoding NAD(P)H:quinone oxidoreductases', Free Radic Biol Med, 29 (3-4), 254-62.

Johnson, Jeffrey A., et al. (2008), 'The Nrf2-ARE Pathway', Annals of the New York Academy of Sciences, 1147 (1), 61-69. 
Jonkers, W., et al. (2016), 'Chemotropism and Cell Fusion in Neurospora crassa Relies on the Formation of Distinct Protein Complexes by HAM-5 and a Novel Protein HAM-14', Genetics, 203 (1), 319-34.

Jonkers, W., et al. (2014), 'HAM-5 functions as a MAP kinase scaffold during cell fusion in Neurospora crassa', PLoS Genet, 10 (11), e1004783.

Kaneko, I., et al. (2006), 'Nonallelic interactions between het-c and a polymorphic locus, pin-c, are essential for nonself recognition and programmed cell death in Neurospora crassa', Genetics, 172 (3), 1545-55.

Kato, N., et al. (2013), 'A point mutation in ftmD blocks the fumitremorgin biosynthetic pathway in Aspergillus fumigatus strain Af293', Biosci Biotechnol Biochem, 77 (5), 1061-7.

Kazmierczak, P., et al. (1996), 'Transcriptional repression of specific host genes by the mycovirus Cryphonectria hypovirus 1', J Virol, 70 (2), 1137-42.

Kazmierczak, P., et al. (2005), 'A Hydrophobin of the chestnut blight fungus, Cryphonectria parasitica, is required for stromal pustule eruption', Eukaryot Cell, 4 (5), 931-6.

Khan, M. A., Chock, P. B., and Stadtman, E. R. (2005), 'Knockout of caspase-like gene, YCA1, abrogates apoptosis and elevates oxidized proteins in Saccharomyces cerevisiae', Proc Natl Acad Sci U S A, 102 (48), 17326-31.

Kibanov, Mikhail V., et al. (2011), 'A novel organelle, the piNG-body, in the nuage of Drosophila male germ cells is associated with piRNA-mediated gene silencing', Molecular Biology of the Cell, 22 (18), 3410-19.

Kicka, Sébastien and Silar, Philippe (2004), 'PaASK1, a Mitogen-Activated Protein Kinase Kinase Kinase That Controls Cell Degeneration and Cell Differentiation in Podospora anserina', Genetics, 166 (3), 1241-52.

Kim, D., et al. (2013), 'TopHat2: accurate alignment of transcriptomes in the presence of insertions, deletions and gene fusions', Genome Biol, 14 (4), R36.

Kitamura, Kenji, et al. (2001), 'Phosphorylation of Mei2 and Ste11 by Pat1 Kinase Inhibits Sexual Differentiation via Ubiquitin Proteolysis and 14-3-3 Protein in Fission Yeast', Developmental Cell, 1 (3), 389-99.

Kitazume, Tatsuya, et al. (2000), 'Fusarium oxysporum Fatty-acid Subterminal Hydroxylase (CYP505) Is a Membrane-bound Eukaryotic Counterpart of Bacillus megaterium Cytochrome P450BM3', Journal of Biological Chemistry, 275 (50), 39734-40.

Kjaerulff, S., et al. (1997), 'Cell differentiation by interaction of two HMG-box proteins: Mat1-Mc activates $\mathrm{M}$ cell-specific genes in S.pombe by recruiting the ubiquitous transcription factor Ste11 to weak binding sites', EMBO J, 16 (13), 4021-33.

Kolling, R. and Hollenberg, C. P. (1994), 'The ABC-transporter Ste6 accumulates in the plasma membrane in a ubiquitinated form in endocytosis mutants', $E M B O J, 13$ (14), 3261-71.

Kuehnle, Katrin, et al. (2008), 'Prosurvival Effect of DHCR24/Seladin-1 in Acute and Chronic Responses to Oxidative Stress', Molecular and Cellular Biology, 28 (2), 539-50.

Kües, Ursula and Casselton, Lorna A. (1992), 'Fungal mating type genes - regulators of sexual development', Mycological Research, 96 (12), 993-1006. 
Lafontaine, D. L. and Smith, M. L. (2012), 'Diverse interactions mediate asymmetric incompatibility by the het-6 supergene complex in Neurospora crassa', Fungal Genet Biol, 49 (1), 65-73.

Lam, E., Kato, N., and Lawton, M. (2001), 'Programmed cell death, mitochondria and the plant hypersensitive response', Nature, 411 (6839), 848-53.

LARROY, Carol, et al. (2002), 'Characterization of the Saccharomyces cerevisiae YMR318C (ADH6) gene product as a broad specificity NADPH-dependent alcohol dehydrogenase: relevance in aldehyde reduction', Biochemical Journal, 361 (1), 163-72.

Larson, T. G. and Nuss, D. L. (1994), 'Altered transcriptional response to nutrient availability in hypovirus-infected chestnut blight fungus', EMBO J, 13 (23), 561623.

LeBel, Carl P., Ischiropoulos, Harry, and Bondy, Stephen C. (1992), 'Evaluation of the probe 2',7'-dichlorofluorescin as an indicator of reactive oxygen species formation and oxidative stress', Chemical Research in Toxicology, 5 (2), 227-31.

Leeder, Abigail C., et al. (2013), 'Early Colony Establishment in $<$ em $>$ Neurospora crassa</em> Requires a MAP Kinase Regulatory Network', Genetics, 195 (3), 883-98.

Leipe, D. D., Koonin, E. V., and Aravind, L. (2004), 'STAND, a class of P-loop NTPases including animal and plant regulators of programmed cell death: multiple, complex domain architectures, unusual phyletic patterns, and evolution by horizontal gene transfer', J Mol Biol, 343 (1), 1-28.

Leslie, John F. and Zeller, Kurt A. (1996), 'Heterokaryon incompatibility in fungi-more than just another way to die', Journal of Genetics, 75 (3), 415-24.

Leulier, F. and Lemaitre, B. (2008), 'Toll-like receptors--taking an evolutionary approach', Nat Rev Genet, 9 (3), 165-78.

Leveau, Johan H. J. and Preston, Gail M. (2008), 'Bacterial mycophagy: definition and diagnosis of a unique bacterial-fungal interaction', New Phytologist, 177 (4), 85976.

Li, Dan, et al. (2005), 'A Mitogen-Activated Protein Kinase Pathway Essential for Mating and Contributing to Vegetative Growth in $<\mathrm{em}>$ Neurospora crassa $</ \mathrm{em}>$ ', Genetics, 170 (3), 1091-104.

Liao, D., et al. (2001), 'Structures of trihydroxynaphthalene reductase-fungicide complexes: implications for structure-based design and catalysis', Structure, 9 (1), $19-27$.

Liu, J., et al. (2009), 'Analysis of Drosophila segmentation network identifies a JNK pathway factor overexpressed in kidney cancer', Science, 323 (5918), 1218-22.

Liu, Yir-Chung and Milgroom, Michael G (1996), 'Correlation between hypovirus transmission and the number of vegetative incompatibility (vic) genes different among isolates from a natural population of Cryphonectria parasitica', Phytopathology, 86 (1), 79-86.

Liu, Yir-Chung and Milgroom, Michael G. (2007), 'High diversity of vegetative compatibility types in Cryphonectria parasitica in Japan and China', Mycologia, 99 (2), 279-84. 
Livak, Kenneth J. and Schmittgen, Thomas D. (2001), 'Analysis of Relative Gene Expression Data Using Real-Time Quantitative PCR and the 2- $\Delta \Delta \mathrm{CT}$ Method', Methods, 25 (4), 402-08.

Lorenzo, H. K., et al. (1999), 'Apoptosis inducing factor (AIF): a phylogenetically old, caspase-independent effector of cell death', Cell Death Differ, 6 (6), 516-24.

Love, M. I., Huber, W., and Anders, S. (2014), 'Moderated estimation of fold change and dispersion for RNA-seq data with DESeq2', Genome Biol, 15 (12), 550.

Lubkowitz, Mark A., et al. (1998), 'Schizosaccharomyces pombe isp4 encodes a transporter representing a novel family of oligopeptide transporters', Molecular Microbiology, 28 (4), 729-41.

Mace, P. D. and Riedl, S. J. (2010), 'Molecular cell death platforms and assemblies', Curr Opin Cell Biol, 22 (6), 828-36.

Madeo, F., et al. (2002), 'A caspase-related protease regulates apoptosis in yeast', Mol Cell, 9 (4), 911-7.

Madeo, Frank, Fröhlich, Eleonore, and Fröhlich, Kai-Uwe (1997), 'A Yeast Mutant Showing Diagnostic Markers of Early and Late Apoptosis', The Journal of Cell Biology, 139 (3), 729-34.

Maeder, C. I., et al. (2007), 'Spatial regulation of Fus3 MAP kinase activity through a reaction-diffusion mechanism in yeast pheromone signalling', Nature Cell Biology, 9 (11), 1319-U226.

Maiya, S., et al. (2007), 'Identification of a hybrid PKS/NRPS required for pseurotin A biosynthesis in the human pathogen Aspergillus fumigatus', Chembiochem, 8 (14), 1736-43.

Markham, Paul and Collinge, Annette J. (1987), 'Woronin bodies of filamentous fungi', FEMS Microbiology Reviews, 3 (1), 1-11.

Marra, R. E. and Milgroom, M. G. (2001), 'The mating system of the fungus Cryphonectria parasitica: selfing and self-incompatibility', Heredity (Edinb), 86 (Pt 2), 134-43.

Masloff, S., et al. (2002), 'Functional analysis of the C6 zinc finger gene pro1 involved in fungal sexual development', Fungal Genet Biol, 36 (2), 107-16.

Mata, Juan and Bähler, Jürg (2006), 'Global roles of Ste11p, cell type, and pheromone in the control of gene expression during early sexual differentiation in fission yeast', Proceedings of the National Academy of Sciences, 103 (42), 15517-22.

Mayorga, M. E. and Timberlake, W. E. (1992), 'The developmentally regulated Aspergillus nidulans wA gene encodes a polypeptide homologous to polyketide and fatty acid synthases', Mol Gen Genet, 235 (2-3), 205-12.

McGuire, I. C., et al. (2001), 'Analysis of mating-type genes in the chestnut blight fungus, Cryphonectria parasitica', Fungal Genet Biol, 34 (2), 131-44.

McLean, K.J., et al. (2005), 'Biodiversity of cytochrome P450 redox systems', Biochemical Society Transactions, 33 (4), 796-801.

Mei, J., et al. (2006), 'The p53-inducible apoptotic protein AMID is not required for normal development and tumor suppression', Oncogene, 25 (6), 849-56.

Merkel, Hermann W (1906), 'A deadly fungus on the American chestnut', Annual Report of the New York Zoological Society, 10, 97-103. 
Metzstein, Mark M., Stanfield, Gillian M., and Horvitz, H. Robert (1998), 'Genetics of programmed cell death in C. elegans: past, present and future', Trends in Genetics, 14 (10), 410-16.

Micali, C. O. and Smith, M. L. (2003), 'On the independence of barrage formation and heterokaryon incompatibility in Neurospora crassa', Fungal Genet Biol, 38 (2), 209-19.

Micali, Cristina O. and Smith, Myron L. (2006), 'A Nonself Recognition Gene Complex in Neurospora crassa', Genetics, 173 (4), 1991-2004.

Milgroom, M. G. and Cortesi, Paolo (1999), 'Analysis of population structure of the chestnut blight fungus based on vegetative incompatibility genotypes', Proceedings of the National Academy of Sciences, 96 (18), 10518-23.

Milgroom, M. G. and Cortesi, P. (2004), 'Biological control of chestnut blight with hypovirulence: a critical analysis', Annu Rev Phytopathol, 42, 311-38.

Modjtahedi, N., et al. (2006), 'Apoptosis-inducing factor: vital and lethal', Trends Cell Biol, 16 (5), 264-72.

Moresco, Eva Marie Y., LaVine, Diantha, and Beutler, Bruce (2011), 'Toll-like receptors', Current Biology, 21 (13), R488-R93.

Neal, G. E., Judah, D. J., and Green, J. A. (1986), 'The in vitro metabolism of aflatoxin B1 catalyzed by hepatic microsomes isolated from control or 3methylcholanthrene-stimulated rats and quail', Toxicol Appl Pharmacol, 82 (3), 454-60.

Niehaus, Eva-Maria, et al. (2013), 'Genetic Manipulation of the Fusarium fujikuroi Fusarin Gene Cluster Yields Insight into the Complex Regulation and Fusarin Biosynthetic Pathway', Chemistry \& Biology, 20 (8), 1055-66.

Nikolova-Karakashian, Mariana, et al. (1997), 'Bimodal Regulation of Ceramidase by Interleukin-1 $\beta$ : IMPLICATIONS FOR THE REGULATION OF CYTOCHROME P450 2C11 (CYP2C11)', Journal of Biological Chemistry, 272 (30), 18718-24.

Noverr, Mairi C., et al. (2001), 'Pathogenic Yeasts Cryptococcus neoformans and Candida albicans Produce Immunomodulatory Prostaglandins', Infection and Immunity, 69 (5), 2957-63.

Nürnberger, Thorsten, et al. (2004), 'Innate immunity in plants and animals: striking similarities and obvious differences', Immunological Reviews, 198 (1), 249-66.

Nuss, D. L. (1996), 'Using Hypoviruses to Probe and Perturb Signal Transduction Processes Underlying Fungal Pathogenesis', The Plant Cell, 8 (10), 1845-53.

O'Neill, L. A. J. and Bowie, A. G. (2007), 'The family of five: TIR-domain-containing adaptors in Toll-like receptor signalling', Nature Reviews Immunology, 7 (5), 353 64.

Obeid, LM, et al. (1993), 'Programmed cell death induced by ceramide', Science, 259 (5102), 1769-71.

Ohta, Hideki, et al. (1994), 'A possible role of sphingosine in induction of apoptosis by tumor necrosis factor- $\alpha$, in human neutrophils', FEBS Letters, 355 (3), 267-70.

Owens, R. A., et al. (2015), 'Interplay between Gliotoxin Resistance, Secretion, and the Methyl/Methionine Cycle in Aspergillus fumigatus', Eukaryot Cell, 14 (9), 94157. 
Owsianowski, Esther, Walter, David, and Fahrenkrog, Birthe (2008), 'Negative regulation of apoptosis in yeast', Biochimica et Biophysica Acta (BBA) - Molecular Cell Research, 1783 (7), 1303-10.

Pandey, A., et al. (2004), 'Role of a mitogen-activated protein kinase pathway during conidial germination and hyphal fusion in Neurospora crassa', Eukaryot Cell, 3 (2), 348-58.

Paoletti, M. and Clavé, C. (2007), 'The Fungus-Specific HET Domain Mediates Programmed Cell Death in Podospora anserina', Eukaryotic Cell, 6 (11), 2001-08.

Paoletti, M. and Saupe, S. J. (2009), 'Fungal incompatibility: evolutionary origin in pathogen defense?', Bioessays, 31 (11), 1201-10.

Paoletti, M., Saupe, S. J., and Clave, C. (2007), 'Genesis of a fungal non-self recognition repertoire', PLoS One, 2 (3), e283.

Park, Hee-Soo and Yu, Jae-Hyuk (2012), 'Genetic control of asexual sporulation in filamentous fungi', Current Opinion in Microbiology, 15 (6), 669-77.

Park, S. M., et al. (2004), 'Characterization of HOG1 homologue, CpMK1, from Cryphonectria parasitica and evidence for hypovirus-mediated perturbation of its phosphorylation in response to hypertonic stress', Mol Microbiol, 51 (5), 1267-77.

Parsley, T. B., et al. (2002), 'Differential modulation of cellular signaling pathways by mild and severe hypovirus strains', Eukaryot Cell, 1 (3), 401-13.

Parsley, Todd B., et al. (2003), 'Analysis of altered G-protein subunit accumulation in Cryphonectria parasitica reveals a third Ga homologue', Current Genetics, 43 (1), 24-33.

Pierce, M., et al. (2003), 'Sum1 and Ndt80 proteins compete for binding to middle sporulation element sequences that control meiotic gene expression', Mol Cell Biol, 23 (14), 4814-25.

Pinan-Lucarré, Bérangère, Paoletti, Mathieu, and Clavé, Corinne (2007), 'Cell death by incompatibility in the fungus Podospora', Seminars in Cancer Biology, 17 (2), 101-11.

Pollier, J., Rombauts, S., and Goossens, A. (2013), 'Analysis of RNA-Seq data with TopHat and Cufflinks for genome-wide expression analysis of jasmonate-treated plants and plant cultures', Methods Mol Biol, 1011, 305-15.

Polyak, K., et al. (1997), 'A model for p53-induced apoptosis', Nature, 389 (6648), 300-5.

Pozniakovsky, A. I., et al. (2005), 'Role of mitochondria in the pheromone- and amiodarone-induced programmed death of yeast', J Cell Biol, 168 (2), 257-69.

Pryer, N K, et al. (1993), 'Cytosolic Sec13p complex is required for vesicle formation from the endoplasmic reticulum in vitro', The Journal of Cell Biology, 120 (4), 865-75.

Qiao, Kangjian, Chooi, Yit-Heng, and Tang, Yi (2011), 'Identification and engineering of the cytochalasin gene cluster from Aspergillus clavatus NRRL 1', Metabolic Engineering, 13 (6), 723-32.

Qin, J., et al. (2003), 'Ste11p, a high-mobility-group box DNA-binding protein, undergoes pheromone- and nutrient-regulated nuclear-cytoplasmic shuttling', $\mathrm{Mol}$ Cell Biol, 23 (9), 3253-64.

Raju, Namboori B. and Perkins, David D. (2000), 'Programmed Ascospore Death in the Homothallic Ascomycete Coniochaeta tetraspora', Fungal Genetics and Biology, $30(3), 213-21$. 
Rampal, Amrit L, Pinkofsky, Harold B, and Jung, Chan Y (1980), 'Structure of cytochalasins and cytochalasin B binding sites in human erythrocyte membranes', Biochemistry, 19 (4), 679-83.

Ray, P. D., Huang, B. W., and Tsuji, Y. (2012), 'Reactive oxygen species (ROS) homeostasis and redox regulation in cellular signaling', Cell Signal, 24 (5), 98190.

Read, Nick D., et al. (2009), 'Self-signalling and self-fusion in filamentous fungi', Current Opinion in Microbiology, 12 (6), 608-15.

Regenberg, Birgitte, et al. (1999), 'Substrate specificity and gene expression of the amino-acid permeases in Saccharomyces cerevisiae', Current Genetics, 36 (6), 317-28.

Riedl, S. J. and Salvesen, G. S. (2007), 'The apoptosome: signalling platform of cell death', Nat Rev Mol Cell Biol, 8 (5), 405-13.

Riek, Roland and Saupe, Sven J. (2016), 'The HET-S/s Prion Motif in the Control of Programmed Cell Death', Cold Spring Harbor Perspectives in Biology, 8 (9).

Roane, M. K., Griffin, G. J., and Elkins, J. R. (1986), 'Chestnut blight, other Endothia diseases, and the genus Endothia', (St. Paul, Minnesota: The American Phytopathological Society), vii $+53 \mathrm{pp}$.

Sato, Takaaki, et al. (1994), 'Interactions among members of the Bcl-2 protein family analyzed with a yeast two-hybrid system', Proceedings of the National Academy of Sciences, 91 (20), 9238-42.

Saupe, Sven J. (2000), 'Molecular Genetics of Heterokaryon Incompatibility in Filamentous Ascomycetes', Microbiology and Molecular Biology Reviews, 64 (3), 489-502.

Scorrano, Luca and Korsmeyer, Stanley J. (2003), 'Mechanisms of cytochrome c release by proapoptotic BCL-2 family members', Biochemical and Biophysical Research Communications, 304 (3), 437-44.

Segers, G. C. and Nuss, D. L. (2003), 'Constitutively activated G $\alpha$ negatively regulates virulence, reproduction and hydrophobin gene expression in the chestnut blight fungus Cryphonectria parasitica', Fungal Genetics and Biology, 38 (2), 198-208.

Segers, G. C., Regier, J. C., and Nuss, D. L. (2004), 'Evidence for a role of the regulator of G-protein signaling protein CPRGS-1 in Galpha subunit CPG-1-mediated regulation of fungal virulence, conidiation, and hydrophobin synthesis in the chestnut blight fungus Cryphonectria parasitica', Eukaryot Cell, 3 (6), 1454-63.

Segers, G. C., et al. (2006), 'Hypovirus papain-like protease p29 suppresses RNA silencing in the natural fungal host and in a heterologous plant system', Eukaryot Cell, 5 (6), 896-904.

Segers, G. C., et al. (2007), 'Evidence that RNA silencing functions as an antiviral defense mechanism in fungi', Proc Natl Acad Sci U S A, 104 (31), 12902-6.

Seuring, Carolin, et al. (2012), 'The Mechanism of Toxicity in HET-S/HET-s Prion Incompatibility', PLOS Biology, 10 (12), e1001451.

Severin, F. F. and Hyman, A. A. (2002), 'Pheromone induces programmed cell death in S. cerevisiae', Curr Biol, 12 (7), R233-5.

Sharon, A., et al. (2009), 'Fungal apoptosis: function, genes and gene function', FEMS Microbiol Rev, 33 (5), 833-54. 
Shiu, Patrick Ka Tai and Glass, N. Louise (1999), 'Molecular Characterization of $<$ em $>$ tol $<$ /em $>$, a Mediator of Mating-Type-Associated Vegetative Incompatibility in $<\mathrm{em}>$ Neurospora crassa</em>', Genetics, 151 (2), 545-55.

Shlezinger, N., Goldfinger, N., and Sharon, A. (2012), 'Apoptotic-like programed cell death in fungi: the benefits in filamentous species', Front Oncol, $2,97$.

Shoun, Hirofumi and Takaya, Naoki (2002), 'Cytochromes P450nor and P450foxy of the fungus Fusarium oxysporum', International Congress Series, 1233, 89-97.

Siomi, H. and Siomi, M. C. (2009), 'On the road to reading the RNA-interference code', Nature, 457 (7228), 396-404.

Smith, M. L. and Lafontaine, D (2013), 'The fungal sense of nonself', Neurospora: Genomics and Molecular Biology, 9-21.

Smith, M. L., Gibbs, C. C., and Milgroom, M. G. (2006), 'Heterokaryon incompatibility function of barrage-associated vegetative incompatibility genes (vic) in Cryphonectria parasitica', Mycologia, 98 (1), 43-50.

Smith, M. L., et al. (2000), 'Vegetative incompatibility in the het-6 region of Neurospora crassa is mediated by two linked genes', Genetics, 155 (3), 1095-104.

Sugimoto, A., et al. (1991), 'Schizosaccharomyces pombe ste11+ encodes a transcription factor with an HMG motif that is a critical regulator of sexual development', Genes Dev, 5 (11), 1990-9.

Sun, Q., Choi, G. H., and Nuss, D. L. (2009a), 'A single Argonaute gene is required for induction of RNA silencing antiviral defense and promotes viral RNA recombination', Proc Natl Acad Sci US A, 106 (42), 17927-32.

--- (2009b), 'Hypovirus-responsive transcription factor gene pro1 of the chestnut blight fungus Cryphonectria parasitica is required for female fertility, asexual spore development, and stable maintenance of hypovirus infection', Eukaryot Cell, 8 (3), 262-70.

Suzuki, N. and Nuss, D. L. (2002), 'Contribution of Protein p40 to Hypovirus-Mediated Modulation of Fungal Host Phenotype and Viral RNA Accumulation', Journal of Virology, 76 (15), 7747-59.

Suzuki, N., Chen, B., and Nuss, D. L. (1999), 'Mapping of a hypovirus p29 protease symptom determinant domain with sequence similarity to potyvirus HC-Pro protease', J Virol, 73 (11), 9478-84.

Suzuki, N., et al. (2003), 'Hypovirus papain-like protease p29 functions in trans to enhance viral double-stranded RNA accumulation and vertical transmission', $J$ Virol, 77 (21), 11697-707.

Tait, S. W. and Green, D. R. (2008), 'Caspase-independent cell death: leaving the set without the final cut', Oncogene, 27 (50), 6452-61.

Talbot, N. J., Ebbole, D. J., and Hamer, J. E. (1993), 'Identification and characterization of MPG1, a gene involved in pathogenicity from the rice blast fungus Magnaporthe grisea', Plant Cell, 5 (11), 1575-90.

Tam, O. H., et al. (2008), 'Pseudogene-derived small interfering RNAs regulate gene expression in mouse oocytes', Nature, 453 (7194), 534-8.

Tanha, Fuad (2008), 'Hypovirus manipulation of nonself recognition-associated programmed cell death in the chestnut blight fungus, Cryphonectria parasitica', (ProQuest Dissertations Publishing). 
Tell, Gianluca, et al. (2008), 'The Many Functions of APE1/Ref-1: Not Only a DNA Repair Enzyme', Antioxidants \& Redox Signaling, 11 (3), 601-19.

Tenney, Karen, et al. (2000), 'hex-1, a Gene Unique to Filamentous Fungi, Encodes the Major Protein of the Woronin Body and Functions as a Plug for Septal Pores', Fungal Genetics and Biology, 31 (3), 205-17.

Tew, Kenneth D. and Townsend, Danyelle M. (2012), 'Glutathione-S-Transferases As Determinants of Cell Survival and Death', Antioxidants \& Redox Signaling, 17 (12), 1728-37.

Thrane, C., et al. (2004), 'Activation of caspase-like activity and poly (ADP-ribose) polymerase degradation during sporulation in Aspergillus nidulans', Fungal Genetics and Biology, 41 (3), 361-68.

Tobiume, Kei, et al. (2001), 'ASK1 is required for sustained activations of JNK/p38 MAP kinases and apoptosis', EMBO reports, 2 (3), 222-28.

Trapnell, C., et al. (2012), 'Differential gene and transcript expression analysis of RNAseq experiments with TopHat and Cufflinks', Nat Protoc, 7 (3), 562-78.

Tsiatsiani, L., et al. (2011), 'Metacaspases', Cell Death Differ, 18 (8), 1279-88.

Tsitsigiannis, Dimitrios I., Zarnowski, Robert, and Keller, Nancy P. (2004), 'The Lipid Body Protein, PpoA, Coordinates Sexual and Asexual Sporulation in Aspergillus nidulans', Journal of Biological Chemistry, 279 (12), 11344-53.

Tsitsigiannis , Dimitrios I., et al. (2005), 'Aspergillus Cyclooxygenase-Like Enzymes Are Associated with Prostaglandin Production and Virulence', Infection and Immunity, 73 (8), 4548-59.

Turina, M., Prodi, A., and Alfen, N. K. (2003), 'Role of the Mf1-1 pheromone precursor gene of the filamentous ascomycete Cryphonectria parasitica', Fungal Genet Biol, $40(3), 242-51$.

Turina, M., Rossi, M., and Moretti, M. (2016), 'Investigation on the partial resistance of Cpkk2 knock out strain of Cryphonectria parasitica to Cryphonectria hypovirus 1 infection in presence of Geneticin and Geneticin resistance gene', Virus Res, 219, 58-61.

Uno, T., Ishizuka, M., and Itakura, T. (2012), 'Cytochrome P450 (CYP) in fish', Environmental Toxicology and Pharmacology, 34 (1), 1-13.

Usui, T., et al. (1998), 'Tryprostatin A, a specific and novel inhibitor of microtubule assembly', Biochem J, 333 ( Pt 3), 543-8.

Vagin, Vasily V., et al. (2004), 'The RNA Interference Proteins and Vasa Locus are Involved in the Silencing of Retrotransposons in the Female Germline of Drosophila melanogaster', RNA Biology, 1 (1), 53-57.

van Diepeningen, A. D., Debets, Alfons J. M., and Hoekstra, Rolf F. (1997), 'Heterokaryon incompatibility blocks virus transfer among natural isolates of black Aspergilli', Current Genetics, 32 (3), 209-17.

Vidal-Cros, Anne, et al. (1994), 'Polyhydroxynaphthalene reductase involved in melanin biosynthesis in Magnaporthe grisea', European Journal of Biochemistry, 219 (3), 985-92.

Wang, Dan-Ni, et al. (2014), 'GliA in Aspergillus fumigatus is required for its tolerance to gliotoxin and affects the amount of extracellular and intracellular gliotoxin', Medical Mycology, 52 (5), 506-18. 
Wichmann, Gale, et al. (2008), 'A novel gene, phcA from Pseudomonas syringae induces programmed cell death in the filamentous fungus Neurospora crassa', Molecular Microbiology, 68 (3), 672-89.

Wissing, S., et al. (2004), 'An AIF orthologue regulates apoptosis in yeast', J Cell Biol, 166 (7), 969-74.

Wu, Jennifer and Glass, N. Louise (2001), 'Identification of Specificity Determinants and Generation of Alleles with Novel Specificity at the het-cHeterokaryon Incompatibility Locus of Neurospora crassa', Molecular and Cellular Biology, 21 (4), 1045-57.

Wu, Min, et al. (2002), 'AMID, an Apoptosis-inducing Factor-homologous Mitochondrion-associated Protein, Induces Caspase-independent Apoptosis', Journal of Biological Chemistry, 277 (28), 25617-23.

$\mathrm{Wu}$, Songsong, et al. (2017), 'Virus-mediated suppression of host non-self recognition facilitates horizontal transmission of heterologous viruses', PLOS Pathogens, 13 (3), e1006234.

Xiang, Q. and Glass, N. L. (2002), 'Identification of vib-1, a locus involved in vegetative incompatibility mediated by het-c in Neurospora crassa', Genetics, 162 (1), 89101 .

--- (2004), 'The control of mating type heterokaryon incompatibility by vib-1, a locus involved in het-c heterokaryon incompatibility in Neurospora crassa', Fungal Genet Biol, 41 (12), 1063-76.

$\mathrm{Xu}$, Jin-Rong, Staiger, Christopher J., and Hamer, John E. (1998), 'Inactivation of the mitogen-activated protein kinase Mps1 from the rice blast fungus prevents penetration of host cells but allows activation of plant defense responses', Proceedings of the National Academy of Sciences, 95 (21), 12713-18.

$\mathrm{Xu}$, Qunli and Reed, John C (1998), 'Bax inhibitor-1, a mammalian apoptosis suppressor identified by functional screening in yeast', Molecular cell, 1 (3), 337-46.

Yin, Z., et al. (2000), 'Glutathione S-transferase p elicits protection against H2O2induced cell death via coordinated regulation of stress kinases', Cancer Res, 60 (15), 4053-7.

Yu, Jiujiang, et al. (2004), 'Clustered Pathway Genes in Aflatoxin Biosynthesis', Applied and Environmental Microbiology, 70 (3), 1253-62.

Zhang, D. X., et al. (2014), 'Vegetative Incompatibility Loci with Dedicated Roles in Allorecognition Restrict Mycovirus Transmission in Chestnut Blight Fungus', Genetics, 197 (2), 701-14.

Zhang, J., et al. (2006a), 'BRG1 interacts with Nrf2 to selectively mediate HO-1 induction in response to oxidative stress', Mol Cell Biol, 26 (21), 7942-52.

Zhang, L., Baasiri, R. A., and Van Alfen, N. K. (1998), 'Viral repression of fungal pheromone precursor gene expression', Mol Cell Biol, 18 (2), 953-9.

Zhang, L., et al. (1994), 'Virus-associated down-regulation of the gene encoding cryparin, an abundant cell-surface protein from the chestnut blight fungus, Cryphonectria parasitica', Gene, 139 (1), 59-64.

Zhang, N.N., et al. (2006b), 'Multiple signaling pathways regulate yeast cell death during the response to mating pheromones', Mol Biol Cell, 17 (8), 3409-22. 
Zhang, X. and Nuss, D. L. (2008), 'A host dicer is required for defective viral RNA production and recombinant virus vector RNA instability for a positive sense RNA virus', Proc Natl Acad Sci U S A, 105 (43), 16749-54.

Zhang, X., et al. (2008), 'Characterization of hypovirus-derived small RNAs generated in the chestnut blight fungus by an inducible DCL-2-dependent pathway', J Virol, 82 (6), 2613-9. 


\section{Appendices}

Table S 1. Hierarchical clustering analysis of differentially expressed genes. Table shows only those clustered DE genes that were mentioned in text.

\begin{tabular}{|c|c|c|c|c|c|c|c|c|c|c|c|c|}
\hline Cluster & $\begin{array}{l}\text { EP155_ } \\
\text { p29con } \\
t\end{array}$ & $\begin{array}{l}\text { EP155 } \\
\text { p29sto } \\
\text { pcont }\end{array}$ & $\begin{array}{l}\text { EP155_ } \\
\text { dcl2co } \\
\text { nt }\end{array}$ & $\begin{array}{l}\text { EP155 } \\
\text { wt_mi } \\
x\end{array}$ & $\begin{array}{l}\text { EP155_- } \\
\text { p29mix }\end{array}$ & $\begin{array}{l}\text { EP155_ } \\
\text { p29sto } \\
\text { pmix }\end{array}$ & $\begin{array}{l}\text { EP155_- } \\
\text { dcl2mi } \\
x\end{array}$ & Mean & CP Id & UP Id & Protein.names UniProt & TrEMBL Id \\
\hline & -2.24 & -1.16 & 0.39 & -0.29 & 1.60 & 1.18 & 0.86 & 0.05 & 342366 & Q1257 & Laccase-1 (EC 1.10.3.2) & M3B9D5 \\
\hline Cluster1 & 0.44 & 0.66 & -8.07 & 0.66 & 1.60 & 0.97 & 3.53 & -0.03 & 358133 & P24665 & Aspergillopepsin-2 (EC 3.4.23.19) (Acid & Q00550 \\
\hline Cluster2 & 0.25 & 0.14 & -1.83 & -2.16 & -2.06 & -2.46 & -2.19 & -1.47 & 100328 & Q304B & Neutral ceramidase (N-CDase) (NCDase) & U7Q0G1 \\
\hline Cluster1 & -0.42 & -0.19 & -0.06 & 1.49 & 2.06 & 1.18 & 1.83 & 0.84 & 74333 & 07750 & Protein argonaute-2 (Argonaute2) (EC & D0U264 \\
\hline Cluster8 & 0.15 & 0.71 & 1.73 & 7.30 & 7.49 & 6.94 & 5.84 & 4.31 & 58765 & Q9Y7Q & Glutathione S-transferase 1 (EC 2.5.1.18) & M7T1S0 \\
\hline Cluster1 & -0.95 & -0.95 & -0.95 & 1.61 & 1.87 & 2.01 & 0.00 & 0.38 & 335314 & P17489 & Laccase-1 (EC 1.10.3.2) & A0A066XFI1 \\
\hline Cluster6 & 0.19 & -0.20 & 0.65 & 4.82 & 5.56 & 3.75 & 7.01 & 3.11 & 32824 & Q0314 & Conidial yellow pigment biosynthesis & B8LUA8 \\
\hline Cluster4 & -0.30 & -0.92 & 0.94 & 4.82 & 5.69 & 6.12 & 4.56 & 2.99 & 333952 & Q53FA & Quinone oxidoreductase PIG3 (EC 1.-.-.-) & C5P864 \\
\hline \multirow[t]{3}{*}{ Cluster9 } & -0.95 & -0.95 & -0.95 & 2.15 & 5.70 & 6.98 & 3.63 & 2.23 & 220966 & P21623 & Spore wall maturation protein DIT1 & R1EEJ7 \\
\hline & 0.10 & 0.16 & 0.02 & -0.05 & 0.11 & 0.01 & -0.15 & 0.03 & 63781 & & & \\
\hline & 0.13 & 0.09 & -0.06 & 0.10 & 0.00 & 0.17 & 0.05 & 0.07 & 103555 & Q9907 & Dual specificity protein kinase FUZ7 (EC & Q6GX81 \\
\hline Cluster2 & 0.53 & 1.71 & -2.52 & -1.47 & -0.94 & -2.47 & -0.77 & -0.85 & 83754 & Q1257 & Laccase-1 (EC 1.10.3.2) & V5G6H1 \\
\hline Cluster9 & 0.69 & 0.81 & 1.49 & 4.31 & 6.63 & 6.01 & 2.67 & 3.23 & 354223 & Q4WA & 6-hydroxytryprostatin B O- & E3QBM5 \\
\hline \multirow[t]{2}{*}{ Cluster8 } & -0.78 & -0.79 & 3.80 & 5.74 & 7.08 & 5.04 & 6.98 & 3.87 & 246669 & Q0035 & Putative HC-toxin efflux carrier TOXA & A0A084QV54 \\
\hline & -0.10 & -0.19 & -0.32 & -0.49 & -0.59 & -0.29 & -0.25 & -0.32 & 66950 & P23561 & Serine/threonine-protein kinase STE11 (EC & F5B5C3 \\
\hline Cluster11 & -0.47 & -0.04 & -0.75 & 13.63 & 14.69 & 14.11 & 13.51 & 7.81 & 85578 & & mf2-1 Pheromone precursor & 014431 \\
\hline Cluster1 & -0.25 & -2.13 & 2.98 & 2.24 & 3.22 & 4.17 & 1.67 & 1.70 & 269861 & Q0071 & Probable sterigmatocystin biosynthesis & G2QA74 \\
\hline Cluster4 & -0.50 & -0.43 & 0.69 & 3.59 & 4.20 & 4.30 & 3.33 & 2.17 & 269746 & Q2771 & Cytochrome P450 2L1 (EC 1.14.14.1) & A0A086NT19 \\
\hline Cluster2 & 0.12 & 0.19 & -1.38 & -1.36 & -1.69 & -2.16 & -1.71 & -1.14 & 348628 & Q0208 & Laccase-4 (EC 1.10.3.2) & F0XJW5 \\
\hline \multirow[t]{3}{*}{ Cluster1 } & -0.73 & -0.86 & 0.21 & 1.30 & 1.20 & 2.07 & 2.43 & 0.80 & 62735 & 09430 & Putative xanthine/uracil permease C887.17 & R8B8Y1 \\
\hline & -1.24 & -0.79 & 3.20 & 0.04 & 0.69 & 1.17 & -0.57 & 0.36 & 327261 & Q96U & Laccase-3 (EC 1.10.3.2) & V9QKE4 \\
\hline & 0.87 & 0.94 & 3.03 & 1.02 & -0.13 & 1.09 & -0.21 & 0.95 & 244696 & Q9UV1 & Heterokaryon incompatibility protein 6, OR & A0A084PD15 \\
\hline \multirow[t]{2}{*}{ Cluster4 } & 0.13 & 0.36 & -1.60 & 2.91 & 4.99 & 4.01 & 3.07 & 1.98 & 86830 & Q0314 & Conidial yellow pigment biosynthesis & N4VF92 \\
\hline & 0.12 & 0.05 & 0.27 & 0.99 & 1.03 & 0.50 & 1.66 & 0.66 & 284549 & Q0822 & Glutathione synthetase (GSH synthetase) & I1RSY1 \\
\hline Cluster7 & -0.28 & -0.84 & 0.49 & 7.87 & 8.26 & 8.30 & 6.93 & 4.39 & 44005 & P35693 & mat-2 - MAT+ sexual cell fertilization- & Q96V09 \\
\hline Cluster8 & -0.60 & -0.45 & -0.45 & 6.51 & 5.87 & 6.53 & 6.88 & 3.47 & 274617 & Q6450 & Cholesterol 7-alpha-monooxygenase (EC & A0A010QSC9 \\
\hline Cluster12 & -0.24 & -3.90 & -2.46 & 5.61 & -0.43 & 6.34 & 4.57 & 1.36 & 254916 & P80869 & Glucose 1-dehydrogenase 2 (EC 1.1.1.47) & V5FR07 \\
\hline Cluster12 & -2.07 & -3.71 & -2.81 & 5.90 & 0.65 & 6.53 & 3.18 & 1.10 & 16824 & & unknown_gene & V5FXH3 \\
\hline
\end{tabular}




\begin{tabular}{|c|c|c|c|c|c|c|c|c|c|c|c|c|}
\hline & -0.13 & -0.19 & -0.41 & -0.27 & -0.30 & -0.20 & 0.34 & -0.17 & 61450 & P38093 & Developmental regulator flbA & Q6B971 \\
\hline Cluster8 & 0.68 & 0.75 & -2.72 & 6.61 & 6.74 & 6.10 & 6.07 & 3.46 & 251671 & Q1261 & Trichodiene oxygenase (EC 1.14.-.-) & G2R1V6 \\
\hline \multirow[t]{3}{*}{ Cluster4 } & 1.22 & 0.19 & 2.60 & 5.20 & 4.52 & 4.23 & 4.88 & 3.26 & 355270 & Q0035 & Putative HC-toxin efflux carrier TOXA & B2W1B6 \\
\hline & -2.30 & -0.89 & 1.05 & 0.36 & 1.62 & -0.06 & -0.18 & -0.06 & 53104 & Q9UV1 & dev3j-Heterokaryon incompatibility & G9MHQ6 \\
\hline & -1.46 & -1.23 & 0.13 & 1.25 & 1.13 & 1.12 & 1.74 & 0.38 & 69386 & Q0396 & Laccase (EC 1.10.3.2) (Benzenediol:oxygen & G2R6J2 \\
\hline \multirow[t]{2}{*}{ Cluster4 } & 0.12 & -0.57 & -1.03 & 3.25 & 3.78 & 3.55 & 3.68 & 1.82 & 285012 & P08965 & Meiosis protein mei2 & M4G5Q9 \\
\hline & 0.18 & 0.10 & -0.71 & -0.07 & -0.07 & -0.31 & 0.00 & -0.13 & 89898 & Q9HF & Guanine nucleotide-binding protein alpha- & Q00581 \\
\hline Cluster2 & 0.17 & -0.74 & 0.75 & -2.53 & -2.15 & -1.03 & -2.58 & -1.16 & 255335 & Q4WA & 6-hydroxytryprostatin B O- & A0A094DB91 \\
\hline \multirow[t]{2}{*}{ Cluster7 } & -0.97 & -0.11 & -2.15 & 6.76 & 6.53 & 6.64 & 8.21 & 3.56 & 345802 & Q9Y8G & Bifunctional P-450:DPH-P450 reductase & R8BP13 \\
\hline & 0.10 & 0.06 & -0.05 & -0.08 & -0.37 & -0.23 & -0.28 & -0.12 & 33349 & & & \\
\hline Cluster1 & -1.14 & -0.66 & 1.62 & 2.40 & 1.69 & 1.79 & 1.75 & 1.06 & 338852 & Q4WA & Nonribosomal peptide synthetase 14 (EC & G4UWQ1 \\
\hline Cluster1 & 0.78 & 0.91 & -8.05 & 0.63 & 1.54 & 0.22 & 3.17 & -0.11 & 354471 & P24665 & Aspergillopepsin-2 (EC 3.4.23.19) (Acid & W9C8C1 \\
\hline Cluster1 & -1.68 & -0.60 & -1.82 & 2.40 & 3.12 & 2.62 & 3.37 & 1.06 & 339471 & Q6368 & 25-hydroxycholesterol 7-alpha-hydroxylase & E9EK22 \\
\hline Cluster1 & 0.57 & 0.54 & 2.86 & 2.64 & 2.89 & 2.42 & 1.20 & 1.88 & 339678 & Q1260 & Probable sterigmatocystin biosynthesis & F7VVP4 \\
\hline Cluster1 & -0.82 & -1.04 & -0.52 & 2.56 & 1.51 & 2.74 & 1.78 & 0.89 & 106275 & Q9P6C & Alcohol dehydrogenase 1 (EC 1.1.1.1) & R8BWA9 \\
\hline Cluster1 & -1.30 & -1.24 & -0.74 & 2.90 & 4.02 & 3.40 & 2.62 & 1.38 & 60905 & Q0035 & Putative HC-toxin efflux carrier TOXA & N4XHD6 \\
\hline Cluster2 & -0.34 & -1.86 & 3.17 & -1.19 & -0.95 & 0.31 & -2.13 & -0.43 & 60079 & Q0314 & Conidial yellow pigment biosynthesis & B2B664 \\
\hline Cluster2 & 0.97 & 0.81 & -1.41 & -1.65 & -1.66 & -1.66 & -2.55 & -1.02 & 240373 & & $\operatorname{dev} 3 g$ & L2FJ84 \\
\hline \multirow[t]{2}{*}{ Cluster4 } & 0.14 & -0.14 & 4.82 & 3.50 & 3.83 & 3.76 & 3.74 & 2.81 & 339052 & Q1257 & Laccase-1 (EC 1.10.3.2) & K2REQ3 \\
\hline & 0.14 & 0.39 & -2.60 & -0.28 & -0.75 & -1.37 & -1.02 & -0.78 & 68157 & Q96U & Laccase-3 (EC 1.10.3.2) & S3D6K1 \\
\hline Cluster3 & 0.53 & 0.25 & -2.50 & -3.62 & -2.89 & -3.21 & -3.25 & -2.10 & 18749 & Q0066 & Putative sterigmatocystin biosynthesis & W3XLG4 \\
\hline Cluster1 & -1.07 & -1.37 & 1.18 & 2.05 & 2.58 & 3.05 & 1.26 & 1.10 & 238641 & Q1D8Y & dev3h - Glutamate--tR ligase (EC 6.1.1.17) & S3CXC9 \\
\hline \multirow[t]{2}{*}{ Cluster1 } & -0.27 & 0.03 & -0.78 & 1.24 & 1.88 & 1.68 & 2.26 & 0.86 & 291163 & Q1260 & Probable sterigmatocystin biosynthesis & N4V1L5 \\
\hline & -0.11 & -0.13 & -1.09 & -1.36 & -1.62 & -1.48 & -1.36 & -1.02 & 91188 & Q9P32 & Transcriptional regulatory protein pro1 & B6V9HO \\
\hline Cluster1 & -0.26 & -0.12 & -0.30 & 2.23 & 2.74 & 2.52 & 2.38 & 1.31 & 259069 & Q1539 & Delta(24)-sterol reductase (EC 1.3.1.72) & R8BKA7 \\
\hline Cluster1 & -0.13 & -0.57 & 2.96 & 2.37 & 1.95 & 1.90 & 5.22 & 1.96 & 346809 & Q9Y8G & Bifunctional P-450:DPH-P450 reductase & B2AMA4 \\
\hline Cluster2 & 0.24 & 0.14 & -1.63 & -2.08 & -2.10 & -2.20 & -2.19 & -1.40 & 346479 & Q1260 & Probable sterigmatocystin biosynthesis & W9H9X6 \\
\hline Cluster2 & -0.42 & -0.27 & -2.95 & -2.41 & -3.67 & -1.49 & -1.49 & -1.81 & 346480 & Q1257 & Laccase-1 (EC 1.10.3.2) & W3WVG1 \\
\hline Cluster1 & -0.33 & -1.02 & 1.08 & 1.62 & 2.38 & 1.26 & 1.64 & 0.95 & 340229 & Q0035 & Putative HC-toxin efflux carrier TOXA & S3CM64 \\
\hline Cluster6 & 0.39 & 0.09 & 0.23 & 3.94 & 4.27 & 2.71 & 6.13 & 2.54 & 285678 & Q8BUE & Apoptosis-inducing factor 2 (EC 1.-.-.-) & G2QTT6 \\
\hline Cluster1 & -0.47 & -1.52 & 0.33 & 1.44 & 1.96 & 2.08 & 1.83 & 0.81 & 261854 & 07495 & Protein argonaute (Cell cycle control & D0U267 \\
\hline Cluster2 & -3.52 & -2.91 & -1.67 & -2.34 & -0.35 & -0.14 & 0.19 & -1.53 & 292843 & Q0314 & Conidial yellow pigment biosynthesis & A1CVNo \\
\hline Cluster1 & -0.32 & -1.17 & -0.15 & 2.67 & 2.71 & 3.18 & 2.42 & 1.33 & 262887 & Q9UV1 & $\operatorname{dev} 3 b$ - Heterokaryon incompatibility & G2YFM4 \\
\hline Cluster4 & 0.38 & -0.80 & 0.77 & 4.70 & 5.25 & 5.63 & 4.22 & 2.88 & 262923 & А9СРТ & Tudor domain-containing protein 1 & H1VA35 \\
\hline Cluster4 & 2.06 & 0.00 & 0.90 & 4.49 & 3.42 & 4.97 & 4.07 & 2.84 & 75073 & Q9VFP & Protein roadkill (Hh-induced MATH and & R7UX04 \\
\hline Cluster1 & 0.22 & -0.33 & 2.62 & 1.40 & 2.77 & 2.20 & 3.23 & 1.73 & 261162 & Q6368 & 25-hydroxycholesterol 7-alpha-hydroxylase & M2N4T6 \\
\hline Cluster4 & -1.83 & -1.76 & 1.44 & 3.28 & 4.63 & 4.48 & 2.74 & 1.85 & 261856 & Q9UV1 & $\operatorname{dev} 3 a-$ Heterokaryon incompatibility & A0A066Y276 \\
\hline Cluster1 & 1.02 & 1.18 & -7.51 & 0.00 & 1.07 & 0.12 & 3.65 & -0.07 & 227470 & & & \\
\hline
\end{tabular}




\begin{tabular}{|c|c|c|c|c|c|c|c|c|c|c|c|c|}
\hline & 2.66 & 2.45 & 0.90 & 1.18 & -0.33 & -0.37 & -0.92 & 0.80 & 73939 & Q6FNV & Protein transport protein SEC13-1 & R8BKV3 \\
\hline Cluster15 & 1.37 & -0.02 & -0.92 & 6.52 & 6.76 & 3.49 & 9.86 & 3.87 & 357090 & Q9Y7Q & Glutathione S-transferase 1 (EC 2.5.1.18) & G2Q0J5 \\
\hline Cluster7 & 1.12 & 1.17 & 0.50 & 8.78 & 8.52 & 7.95 & 8.27 & 5.19 & 263100 & Q9F72 & Q2UIJ9 & \\
\hline Cluster8 & -0.63 & 0.04 & -1.55 & 5.69 & 5.68 & 5.97 & 6.58 & 3.11 & 51413 & P40900 & Sexual differentiation process protein isp4 & G3J976 \\
\hline Cluster1 & 0.53 & 0.30 & -9.48 & 0.45 & 1.11 & 0.09 & 3.71 & -0.47 & 242078 & P24665 & Aspergillopepsin-2 (EC 3.4.23.19) (Acid & W3XGK4 \\
\hline Cluster4 & 0.13 & -0.33 & 2.44 & 3.00 & 4.47 & 2.98 & 4.12 & 2.40 & 10611 & Q4WV & Nonribosomal peptide synthetase 8 (EC & X0JE17 \\
\hline Cluster4 & 0.53 & -1.64 & 3.62 & 2.42 & 4.40 & 4.31 & 4.30 & 2.56 & 262538 & Q1261 & Trichodiene oxygenase (EC 1.14.-.-) & W3XLNO \\
\hline Cluster1 & -0.83 & -0.99 & -0.10 & -0.01 & 1.61 & 2.13 & -0.97 & 0.12 & 260952 & Q2UN8 & Metacaspase-1A (EC 3.4.22.-) & A0A066XHH4 \\
\hline Cluster1 & 0.32 & 0.86 & 0.03 & 1.62 & 2.02 & 1.61 & 1.50 & 1.14 & 59909 & Q0314 & Conidial yellow pigment biosynthesis & А0А093Y7H6 \\
\hline Cluster4 & -0.95 & -0.95 & 1.20 & 4.78 & 4.72 & 5.04 & 3.42 & 2.47 & 264062 & P18773 & Esterase (EC 3.1.1.-) & T5APL5 \\
\hline Cluster2 & 0.91 & 0.24 & -2.18 & -3.11 & -3.70 & -2.95 & -0.52 & -1.62 & 332509 & Q4WY8 & Linoleate 10R-lipoxygenase (EC 1.13.11.62) & A0A017SJ74 \\
\hline Cluster1 & -1.43 & -2.41 & 1.06 & -0.02 & 1.79 & 2.79 & 0.68 & 0.35 & 243920 & Q9UV1 & $\operatorname{dev} 3 i-$ Heterokaryon incompatibility & TOKFS6 \\
\hline Cluster4 & 0.22 & 0.45 & -0.79 & 4.52 & 4.93 & 4.37 & 4.78 & 2.64 & 96416 & P12866 & Alpha-factor-transporting ATPase (EC & K3V9K3 \\
\hline Cluster2 & -4.41 & -3.40 & -0.17 & -2.84 & -0.53 & 0.13 & -0.47 & -1.67 & 357339 & 09515 & Aflatoxin B1 aldehyde reductase member 3 & A0A024SHG5 \\
\hline Cluster1 & 1.14 & -1.52 & -0.62 & 0.67 & -0.13 & 1.72 & 3.06 & 0.62 & 264580 & Q0489 & DP-dependent alcohol dehydrogenase 6 & W3WGQ9 \\
\hline Cluster1 & 0.69 & -0.95 & 0.50 & 1.61 & 1.84 & 3.33 & -0.72 & 0.90 & 265800 & P21623 & Spore wall maturation protein DIT1 & A0A010RSH4 \\
\hline \multirow[t]{2}{*}{ Cluster1 } & -0.78 & -0.89 & -0.82 & 3.34 & 3.90 & 3.68 & 2.62 & 1.58 & 342071 & Q0071 & Probable sterigmatocystin biosynthesis & W3WIV8 \\
\hline & 0.05 & 0.12 & -0.29 & -0.16 & -0.30 & -0.33 & -0.13 & -0.15 & 99781 & Q7RZD & Serine/threonine-protein kinase ste20 (EC & R8BWI8 \\
\hline Cluster1 & 0.00 & 1.08 & 0.90 & 3.08 & 3.27 & 1.80 & 3.04 & 1.88 & 47533 & Q0489 & DP-dependent alcohol dehydrogenase 6 & G2QGG1 \\
\hline \multirow{2}{*}{ Cluster1 } & -0.09 & 0.36 & 0.31 & 1.42 & 1.99 & 1.95 & 3.77 & 1.39 & 268419 & Q0080 & $\operatorname{dev} 3 c-$ Vegetative incompatibility protein & A0A094GDJ1 \\
\hline & -0.09 & -0.25 & 0.12 & -0.33 & -0.34 & -0.43 & -0.49 & -0.26 & 103377 & Q0542 & Guanine nucleotide-binding protein alpha- & Q8TF78 \\
\hline Cluster4 & -0.05 & 0.39 & 0.49 & 3.47 & 4.04 & 3.68 & 3.75 & 2.25 & 337117 & P21334 & Phytoene desaturase (EC 1.3.99.30) & W3WXG0 \\
\hline Cluster2 & 0.08 & 0.55 & -5.68 & -1.84 & -2.00 & -2.94 & -1.14 & -1.85 & 97307 & 01403 & Glutathione transporter 1 & N4VPW8 \\
\hline Cluster1 & 0.05 & -0.96 & -0.11 & 1.22 & 1.53 & 2.62 & 1.36 & 0.82 & 336241 & Q9HD & Calcium-transporting ATPase 2 (EC 3.6.3.8) & A0A084QKG7 \\
\hline Cluster1 & -0.01 & 0.11 & 0.49 & 2.70 & 3.01 & 2.98 & 2.65 & 1.70 & 351932 & P36631 & Transcription factor ste11 & TOLZ92 \\
\hline \multirow[t]{6}{*}{ Cluster1 } & -0.07 & -0.47 & -0.67 & 2.12 & 2.46 & 2.49 & 2.17 & 1.15 & 258862 & & vicla & W119Y2 \\
\hline & -0.04 & -0.06 & -0.55 & -0.66 & -0.72 & -0.55 & -0.50 & -0.44 & 33424 & P25344 & Protein STE50 & M4G614 \\
\hline & 0.05 & 0.02 & -0.96 & -0.90 & -1.05 & -0.79 & -0.54 & -0.60 & 86923 & 07425 & Transcription factor steA & Q1PAG9 \\
\hline & 0.05 & 0.03 & -0.19 & -0.01 & -0.10 & -0.16 & 0.02 & -0.05 & 35687 & & & \\
\hline & 0.06 & -0.06 & -1.45 & -1.61 & -1.53 & -1.47 & -1.38 & -1.06 & 67224 & Q9C2N & cpvib1 - Transcription factor vib-1 & C7YTT8 \\
\hline & 0.05 & 0.09 & -0.38 & -0.24 & -0.28 & -0.41 & -0.13 & -0.18 & 105373 & 01443 & Guanine nucleotide-binding protein & M7S9Y1 \\
\hline Cluster7 & 0.00 & 0.00 & 1.45 & 7.90 & 8.37 & 8.43 & 7.31 & 4.78 & 227067 & Q2GM & Plasma membrane fusion protein PRM1 & B2ACV5 \\
\hline Cluster4 & -0.10 & 0.03 & -0.92 & 4.59 & 4.26 & 3.30 & 3.54 & 2.10 & 48444 & Q9UV1 & pseudo-vic - Heterokaryon incompatibility & W11719 \\
\hline Cluster4 & 0.00 & 0.00 & 0.00 & 3.04 & 3.17 & 3.04 & 4.37 & 1.95 & 66954 & P55211 & Caspase-9 (CASP-9) (EC 3.4.22.62) & \\
\hline Cluster2 & -0.60 & -0.08 & -0.99 & -1.26 & -0.96 & -1.22 & -3.43 & -1.22 & NOV_0 & B4PRJ9 & & \\
\hline \multirow[t]{2}{*}{ Cluster5 } & -0.28 & -0.99 & 0.12 & 9.50 & 10.50 & 10.66 & 9.23 & 5.53 & NOV_0 & & Mating type 2 pheromone (probably mf2- & 014431 \\
\hline & -0.06 & -0.05 & -1.35 & -1.06 & -1.24 & -1.64 & -0.98 & -0.91 & NOV_O & Q86BY & cpham-5, HAM-5 ortholog & M4FLD9 \\
\hline
\end{tabular}


Table S 2. DAVID enrichment analysis groups of GO terms and INTERPRO/Pfam domains.

\begin{tabular}{|c|c|c|c|c|c|c|c|c|c|c|c|c|c|c|}
\hline \multirow[b]{2}{*}{$\begin{array}{r}\text { Upregulated gene } \\
\text { groups }\end{array}$} & \multirow[b]{2}{*}{ GO id } & \multirow[b]{2}{*}{$\begin{array}{l}\text { GO term } \\
\text { description }\end{array}$} & \multicolumn{3}{|c|}{ EP155_wt } & \multicolumn{3}{|c|}{ EP155p29 } & \multicolumn{3}{|c|}{ EP155p29 } & \multicolumn{3}{|c|}{ EP155 $1 \mathrm{ddcl}$} \\
\hline & & & $\begin{array}{c}\text { Co } \\
\text { un } \\
t\end{array}$ & Pvalue & $\begin{array}{l}\text { Fold.Enr } \\
\text { ichment }\end{array}$ & $\begin{array}{c}\text { Co } \\
\text { un } \\
t\end{array}$ & Pvalue & $\begin{array}{l}\text { Fold } \\
\text {.Enri } \\
\text { chm } \\
\text { ent }\end{array}$ & $\begin{array}{c}\text { Co } \\
\text { un } \\
t\end{array}$ & Pvalue & $\begin{array}{l}\text { Fold } \\
\text {.Enri } \\
\text { chm } \\
\text { ent }\end{array}$ & $\begin{array}{c}\text { Co } \\
\text { un } \\
t\end{array}$ & Pvalue & $\begin{array}{l}\text { Fold } \\
\text {.Enri } \\
\text { chm } \\
\text { ent }\end{array}$ \\
\hline \multirow{6}{*}{$\begin{array}{r}\text { Alkaloid/ROS } \\
\text { generation }\end{array}$} & GO:0009820 & alkaloid metabolic & & & & 6 & 0.070342 & 2.66 & & & & 5 & 0.200956 & 2.14 \\
\hline & GO:0006739 & NADP metabolic & & & & 3 & 0.298812 & 2.73 & & & & 3 & 0.313868 & 2.64 \\
\hline & GO:0046496 & nicotinamide & & & & 3 & 0.582974 & 1.55 & & & & 3 & 0.602975 & 1.49 \\
\hline & GO:0006769 & nicotinamide & & 、 & & 3 & 0.582974 & 1.55 & & & & 3 & 0.602975 & 1.49 \\
\hline & GO:0006733 & oxidoreduction & & & & 4 & 0.589272 & 1.32 & & & & & & \\
\hline & GO:0043603 & cellular amide & & & & & & & & & & 5 & 0.397543 & 1.56 \\
\hline \multirow{7}{*}{$\begin{array}{r}\text { Amino Acid } \\
\text { Transport }\end{array}$} & GO:0006865 & amino acid & 4 & 0.38755 & 1.78449 & 6 & 0.17009 & 2.02 & 7 & 0.07912 & 2.30 & 10 & 0.002796 & 3.25 \\
\hline & GO:0015837 & amine transport & 4 & 0.563118 & 1.36811 & 8 & 0.087559 & 2.07 & 11 & 0.005247 & 2.77 & 16 & $5.43 \mathrm{E}-06$ & 3.99 \\
\hline & GO:0046942 & carboxylic acid & 4 & 0.585674 & 1.32398 & 6 & 0.366954 & 1.50 & 7 & 0.222138 & 1.70 & 10 & 0.020227 & 2.41 \\
\hline & GO:0015849 & organic acid & 4 & 0.618092 & 1.26287 & 6 & 0.40607 & 1.43 & 8 & 0.133674 & 1.86 & 11 & 0.009928 & 2.53 \\
\hline & GO:0015846 & polyamine & & & & & & & & & & 7 & 0.000248 & 6.99 \\
\hline & GO:0015848 & spermidine & & & & & & & & & & 3 & 0.074153 & 6.42 \\
\hline & GO:0000296 & spermine transport & & & & & & & & & & 3 & 0.074153 & 6.42 \\
\hline \multirow{8}{*}{$\begin{array}{l}\text { Aminoacids } \\
\text { Biosynthesis }\end{array}$} & GO:0042398 & cellular amino acid & 6 & 0.164323 & 2.05217 & 5 & 0.543817 & 1.29 & 6 & 0.361517 & 1.51 & 4 & 0.772521 & 0.99 \\
\hline & GO:0006575 & cellular amino acid & 9 & 0.175262 & 1.64906 & 7 & 0.734798 & 0.97 & 10 & 0.317428 & 1.35 & 10 & 0.32755 & 1.33 \\
\hline & GO:0042439 & ethanolamine and & 3 & 0.307845 & 2.67674 & 3 & 0.43994 & 2.02 & 3 & 0.453554 & 1.97 & & & \\
\hline & GO:0006576 & biogenic amine & 4 & 0.503638 & 1.49249 & 4 & 0.695665 & 1.12 & 4 & 0.712784 & 1.10 & & & \\
\hline & GO:0042401 & biogenic amine & 3 & 0.527583 & 1.71014 & 3 & 0.682742 & 1.29 & 3 & 0.696868 & 1.26 & & & \\
\hline & GO:0019748 & secondary & & & & & & & & & & 20 & $2.28 \mathrm{E}-06$ & 3.48 \\
\hline & GO:0009698 & phenylpropanoid & & & & & & & & & & 7 & 0.000546 & 6.16 \\
\hline & GO:0009699 & phenylpropanoid & & & & & & & & & & 3 & 0.094636 & 5.61 \\
\hline \multirow{3}{*}{$\begin{array}{r}\text { Cell Wall } \\
\text { Development/Growt } \\
h\end{array}$} & GO:0000271 & polysaccharide & 7 & 0.048674 & 2.61185 & 7 & 0.138618 & 1.97 & 7 & 0.151356 & 1.92 & 8 & 0.070141 & 2.17 \\
\hline & GO:0005976 & polysaccharide & 10 & 0.067233 & 1.95445 & 11 & 0.134651 & 1.62 & 13 & 0.042007 & 1.87 & 12 & 0.087761 & 1.71 \\
\hline & GO:0045229 & external. & 9 & 0.072755 & 2.02962 & 11 & 0.064949 & 1.87 & 11 & 0.074646 & 1.82 & & & \\
\hline
\end{tabular}




\begin{tabular}{|c|c|c|c|c|c|c|c|c|c|c|c|c|c|c|}
\hline & GO:0030203 & glycosaminoglycan & 4 & 0.097754 & 3.56899 & & & & 4 & 0.190335 & 2.63 & 5 & 0.06239 & 3.25 \\
\hline & GO:0006022 & aminoglycan & 5 & 0.102268 & 2.77320 & & & & & & & & & \\
\hline & GO:0042546 & cell wall biogenesis & & & & 6 & 0.110467 & 2.32 & & & & 7 & 0.04669 & 2.62 \\
\hline & GO:0007047 & cell wall & & & & 10 & 0.121474 & 1.72 & 10 & 0.136347 & 1.68 & & & \\
\hline & GO:0009273 & peptidoglycan- & & & & & & & & & & 4 & 0.099168 & 3.52 \\
\hline \multirow{7}{*}{$\begin{array}{r}\text { Cellular Ion } \\
\text { Homeostasis }\end{array}$} & GO:0050801 & ion homeostasis & 6 & 0.337314 & 1.55861 & 8 & 0.242354 & 1.57 & & & & 6 & 0.612057 & 1.13 \\
\hline & GO:0055080 & cation homeostasis & 5 & 0.354507 & 1.65497 & 7 & 0.205222 & 1.75 & 7 & 0.222138 & 1.70 & 5 & 0.599192 & 1.20 \\
\hline & GO:0048878 & chemical & 6 & 0.396064 & 1.44859 & & & & & & & 6 & 0.676796 & 1.05 \\
\hline & GO:0055082 & cellular chemical & 5 & 0.410417 & 1.53147 & 7 & 0.258593 & 1.62 & 8 & 0.149846 & 1.80 & 5 & 0.660783 & 1.11 \\
\hline & GO:0006873 & cellular ion & 5 & 0.410417 & 1.53147 & 7 & 0.258593 & 1.62 & 8 & 0.149846 & 1.80 & 5 & 0.660783 & 1.11 \\
\hline & GO:0007267 & cell-cell signaling & & & & 3 & 0.177517 & 3.88 & 3 & 0.184952 & 3.78 & & & \\
\hline & GO:0030003 & cellular cation & & & & & & & 7 & 0.180364 & 1.82 & & & \\
\hline \multirow{6}{*}{$\begin{array}{r}\text { Cofactor } \\
\text { Biosynthesis }\end{array}$} & GO:0006779 & porphyrin & 3 & 0.527583 & 1.71014 & 4 & 0.40983 & 1.72 & 4 & 0.42658 & 1.68 & 5 & 0.215144 & 2.08 \\
\hline & GO:0006778 & porphyrin & 3 & 0.527583 & 1.71014 & 4 & 0.40983 & 1.72 & 4 & 0.42658 & 1.68 & 5 & 0.215144 & 2.08 \\
\hline & GO:0033013 & tetrapyrrole & 3 & 0.54251 & 1.66392 & 4 & 0.427367 & 1.67 & 4 & 0.444411 & 1.63 & 6 & 0.095904 & 2.42 \\
\hline & GO:0033014 & tetrapyrrole & 3 & 0.54251 & 1.66392 & 4 & 0.427367 & 1.67 & 4 & 0.444411 & 1.63 & 6 & 0.095904 & 2.42 \\
\hline & GO:0051188 & cofactor & 8 & 0.546626 & 1.14806 & 11 & 0.437262 & 1.19 & 10 & 0.607312 & 1.05 & & & \\
\hline & GO:0051186 & cofactor metabolic & & & & & & & & & & 15 & 0.454448 & 1.12 \\
\hline \multirow{10}{*}{$\begin{array}{r}\text { DNA } \\
\text { Repair/Response to } \\
\text { Abiotic Factor }\end{array}$} & GO:0031668 & cellular response to & 5 & 0.172482 & 2.28019 & 8 & 0.023099 & 2.76 & 6 & 0.171993 & 2.01 & 6 & 0.176822 & 1.99 \\
\hline & GO:0009432 & SOS response & 3 & 0.180428 & 3.84782 & & & & 3 & 0.284732 & 2.83 & & & \\
\hline & GO:0009991 & response to & 5 & 0.265645 & 1.90016 & 8 & 0.055375 & 2.30 & 6 & 0.281986 & 1.68 & 7 & 0.146873 & 1.94 \\
\hline & GO:0006974 & response to DNA & 6 & 0.983887 & 0.56481 & & & & & & & & & \\
\hline & GO:0006281 & DNA repair & 5 & 0.98862 & 0.52085 & & & & & & & & & \\
\hline & GO:0031669 & cellular response to & & & & 5 & 0.091083 & 2.87 & 3 & 0.539509 & 1.68 & 4 & 0.266114 & 2.21 \\
\hline & GO:0009267 & cellular response to & & & & 4 & 0.133192 & 3.10 & & & & & & \\
\hline & GO:0042594 & response to & & & & 4 & 0.164171 & 2.82 & & & & & & \\
\hline & GO:0031667 & response to & & & & & & & 3 & 0.696868 & 1.26 & 5 & 0.215144 & 2.08 \\
\hline & GO:0007584 & response to & & & & & & & & & & 3 & 0.263247 & 2.99 \\
\hline \multirow[t]{2}{*}{ Glycolysis } & GO:0006007 & glucose catabolic & 4 & 0.253053 & 2.28019 & 6 & 0.077605 & 2.58 & 3 & 0.696868 & 1.26 & 5 & 0.215144 & 2.08 \\
\hline & GO:0019320 & hexose catabolic & 4 & 0.279743 & 2.16018 & 6 & 0.093289 & 2.45 & & & & 5 & 0.244285 & 1.97 \\
\hline
\end{tabular}




\begin{tabular}{|c|c|c|c|c|c|c|c|c|c|c|c|c|c|c|}
\hline & GO:0046365 & monosaccharide & 4 & 0.360714 & 1.86561 & 6 & 0.14897 & 2.11 & & & & 5 & 0.33563 & 1.70 \\
\hline & GO:0006096 & glycolysis & 3 & 0.379671 & 2.28019 & & & & 3 & 0.539509 & 1.68 & & & \\
\hline & GO:0046164 & alcohol catabolic & 4 & 0.515868 & 1.46583 & 7 & 0.147437 & 1.94 & 4 & 0.724294 & 1.08 & 6 & 0.315342 & 1.60 \\
\hline & GO:0044275 & cellular & & & & 8 & 0.121015 & 1.91 & 6 & 0.428369 & 1.39 & 6 & 0.4367 & 1.38 \\
\hline & GO:0016052 & carbohydrate & & & & & & & 8 & 0.544297 & 1.15 & & & \\
\hline \multirow[t]{5}{*}{ Lipid Biosynthesis } & GO:0008610 & lipid biosynthetic & 16 & 0.042964 & 1.71909 & & & & 18 & 0.123914 & 1.42 & & & \\
\hline & GO:0008654 & phospholipid & 7 & 0.122332 & 2.05217 & 7 & 0.292246 & 1.55 & 8 & 0.175721 & 1.72 & & & \\
\hline & GO:0019637 & organophosphate & 9 & 0.187386 & 1.62013 & 9 & 0.450598 & 1.22 & 12 & 0.128683 & 1.59 & & & \\
\hline & GO:0006644 & phospholipid & 8 & 0.269241 & 1.52012 & 9 & 0.390679 & 1.29 & 11 & 0.172239 & 1.54 & & & \\
\hline & GO:0006650 & glycerophospholipid & 4 & 0.574488 & 1.34568 & & & & & & & & & \\
\hline \multirow[t]{14}{*}{ NTPs Biosynthesis } & GO:0006164 & purine nucleotide & 3 & 0.881607 & 0.83196 & & & & & & & & & \\
\hline & GO:0006163 & purine nucleotide & 3 & 0.91411 & 0.75079 & & & & & & & & & \\
\hline & GO:0009165 & nucleotide & 4 & 0.945288 & 0.66199 & & & & & & & & & \\
\hline & GO:0034404 & nucleobase, & 4 & 0.979722 & 0.54724 & & & & & & & & & \\
\hline & GO:0034654 & nucleobase, & 4 & 0.979722 & 0.54724 & & & & & & & & & \\
\hline & GO:0006811 & ion transport & & & & 15 & 0.166208 & 1.42 & & & & & & \\
\hline & GO:0006812 & cation transport & & & & 11 & 0.280464 & 1.36 & & & & & & \\
\hline & GO:0015672 & monovalent & & & & 6 & 0.327908 & 1.57 & & & & & & \\
\hline & GO:0015992 & proton transport & & & & 4 & 0.374306 & 1.82 & & & & 3 & 0.671515 & 1.32 \\
\hline & GO:0006818 & hydrogen transport & & & & 4 & 0.392133 & 1.77 & & & & & & \\
\hline & GO:0015986 & ATP synthesis & & & & & & & & & & 3 & 0.502935 & 1.79 \\
\hline & GO:0015985 & energy coupled & & & & & & & & & & 3 & 0.502935 & 1.79 \\
\hline & GO:0034220 & ion transmembrane & & & & & & & & & & 4 & 0.503058 & 1.49 \\
\hline & GO:0006119 & oxidative & & & & & & & & & & 3 & 0.584241 & 1.55 \\
\hline \multirow[t]{4}{*}{ Peptide Transport } & GO:0008104 & protein localization & 9 & 0.995832 & 0.53380 & 8 & 0.99998 & 0.35 & 9 & 0.999952 & 0.39 & 11 & 0.999595 & 0.47 \\
\hline & GO:0015031 & protein transport & 6 & 0.998299 & 0.44132 & 6 & 0.999957 & 0.33 & 7 & 0.999876 & 0.37 & 9 & 0.99879 & 0.48 \\
\hline & GO:0045184 & establishment of & 6 & 0.998705 & 0.43052 & 6 & 0.999971 & 0.32 & 7 & 0.999915 & 0.37 & 9 & 0.999137 & 0.47 \\
\hline & GO:0015833 & peptide transport & & & & & & & & & & 6 & 0.000677 & 7.49 \\
\hline \multirow{2}{*}{$\begin{array}{r}\text { Pigment } \\
\text { Biosynthesis/Sporul }\end{array}$} & GO:0006721 & terpenoid & 4 & 0.097754 & 3.56899 & & & & 5 & 0.060615 & 3.28 & 5 & 0.06239 & 3.25 \\
\hline & GO:0016114 & terpenoid & 3 & 0.198309 & 3.62148 & & & & & & & 3 & 0.313868 & 2.64 \\
\hline
\end{tabular}




\begin{tabular}{|c|c|c|c|c|c|c|c|c|c|c|c|c|c|c|}
\hline \multirow[t]{9}{*}{ ation } & GO:0006720 & isoprenoid & 4 & 0.239851 & 2.34534 & & & & & & & 5 & 0.200956 & 2.14 \\
\hline & GO:0008299 & isoprenoid & 3 & 0.414441 & 2.12293 & & & & & & & 3 & 0.584241 & 1.55 \\
\hline & GO:0006582 & melanin metabolic & & & & 3 & 0.022603 & 11.6 & 3 & 0.023758 & 11.3 & & & \\
\hline & GO:0016108 & tetraterpenoid & & & & 3 & 0.022603 & 11.6 & & & & & & \\
\hline & GO:0016109 & tetraterpenoid & & & & 3 & 0.022603 & 11.6 & & & & & & \\
\hline & GO:0016117 & carotenoid & & & & 3 & 0.022603 & 11.6 & & & & & & \\
\hline & GO:0042438 & melanin & & & & 3 & 0.022603 & 11.6 & 3 & 0.023758 & 11.3 & & & \\
\hline & GO:0042440 & pigment metabolic & & & & & & & 6 & 0.092933 & 2.45 & & & \\
\hline & GO:0046148 & pigment & & & & & & & 6 & 0.092933 & 2.45 & & & \\
\hline \multirow{5}{*}{$\begin{array}{r}\text { Regulation of } \\
\text { Apoptosis }\end{array}$} & GO:0042981 & regulation of & 8 & 0.000835 & 4.97496 & 8 & 0.0042 & 3.76 & 9 & 0.001052 & 4.12 & 9 & 0.001121 & 4.08 \\
\hline & GO:0043067 & regulation of & 8 & 0.000835 & 4.97496 & 8 & 0.0042 & 3.76 & 9 & 0.001052 & 4.12 & 9 & 0.001121 & 4.08 \\
\hline & GO:0010941 & regulation of cell & 8 & 0.001009 & 4.82864 & 8 & 0.005005 & 3.65 & 9 & 0.001299 & 4.00 & 9 & 0.001383 & 3.96 \\
\hline & GO:0043068 & positive regulation & 5 & 0.011759 & 5.40045 & 5 & 0.029957 & 4.08 & 5 & 0.032584 & 3.98 & 5 & 0.033603 & 3.94 \\
\hline & GO:0043065 & positive regulation & 5 & 0.011759 & 5.40045 & 5 & 0.029957 & 4.08 & 5 & 0.032584 & 3.98 & 5 & 0.033603 & 3.94 \\
\hline \multirow{5}{*}{$\begin{array}{r}\text { Response to } \\
\text { Oxidative Stress }\end{array}$} & GO:0042542 & response to & & & & & & & 4 & 0.083789 & 3.78 & & & \\
\hline & GO:0006979 & response to & & & & & & & 8 & 0.104135 & 1.98 & & & \\
\hline & GO:0070301 & cellular response to & & & & & & & 3 & 0.160783 & 4.12 & & & \\
\hline & GO:0042744 & hydrogen peroxide & & & & & & & 3 & 0.160783 & 4.12 & & & \\
\hline & GO:0000302 & response to & & & & & & & 4 & 0.190335 & 2.63 & & & \\
\hline \multirow[t]{8}{*}{ RNA interference } & GO:0016458 & gene silencing & 6 & 0.046467 & 3.00318 & 7 & 0.044768 & 2.65 & 5 & 0.283495 & 1.84 & 3 & 0.768129 & 1.09 \\
\hline & GO:0006342 & chromatin silencing & 5 & 0.054964 & 3.42028 & 5 & 0.122782 & 2.58 & 4 & 0.316611 & 2.01 & & & \\
\hline & GO:0045814 & negative regulation & 5 & 0.054964 & 3.42028 & 5 & 0.122782 & 2.58 & 4 & 0.316611 & 2.01 & & & \\
\hline & GO:0040029 & regulation of gene & 6 & 0.093007 & 2.46260 & 7 & 0.098523 & 2.17 & 5 & 0.420899 & 1.51 & 3 & 0.855419 & 0.89 \\
\hline & GO:0010629 & negative regulation & 8 & 0.096471 & 2.02683 & 9 & 0.146405 & 1.72 & & & & 5 & 0.797502 & 0.92 \\
\hline & GO:0006476 & protein amino acid & & & & & & & 3 & 0.334614 & 2.52 & & & \\
\hline & GO:0016481 & negative regulation & & & & & & & & & & 4 & 0.847716 & 0.86 \\
\hline & GO:0045892 & negative regulation & & & & & & & & & & 3 & 0.92951 & 0.71 \\
\hline \multirow{3}{*}{$\begin{array}{r}\text { Sexual } \\
\text { Reproduction/Sporu } \\
\text { lation }\end{array}$} & GO:0007283 & spermatogenesis & 4 & 0.046323 & 4.82864 & 3 & 0.298812 & 2.73 & 4 & 0.096989 & 3.55 & & & \\
\hline & GO:0048232 & male gamete & 4 & 0.046323 & 4.82864 & 3 & 0.298812 & 2.73 & 4 & 0.096989 & 3.55 & & & \\
\hline & GO:0007281 & germ cell & 3 & 0.054082 & 7.69565 & & & & 3 & 0.093074 & 5.67 & & & \\
\hline
\end{tabular}




\begin{tabular}{|c|c|c|c|c|c|c|c|c|c|c|c|c|c|c|}
\hline & GO:0019953 & sexual reproduction & 9 & 0.106097 & 1.86561 & 8 & 0.452927 & 1.25 & 9 & 0.327261 & 1.37 & 9 & 0.336883 & 1.36 \\
\hline & GO:0007276 & gamete generation & 4 & 0.1885 & 2.64796 & 3 & 0.601085 & 1.50 & & & & & & \\
\hline & GO:0003006 & reproductive & & & & 8 & 0.431536 & 1.28 & 9 & 0.307248 & 1.40 & 9 & 0.316559 & 1.39 \\
\hline & GO:0048610 & reproductive & & & & & & & & & & 5 & 0.754678 & 0.98 \\
\hline & GO:0043934 & sporulation & & & & & & & & & & 5 & 0.624612 & 1.17 \\
\hline & GO:0030435 & sporulation & & & & & & & & & & 5 & 0.624612 & 1.17 \\
\hline \multirow[t]{4}{*}{ Sterol Metabolism } & GO:0008203 & cholesterol & 3 & 0.252915 & 3.07826 & 3 & 0.37093 & 2.32 & 3 & 0.383439 & 2.26 & 4 & 0.143993 & 2.99 \\
\hline & GO:0016125 & sterol metabolic & 3 & 0.673454 & 1.30989 & 4 & 0.589272 & 1.32 & 3 & 0.826157 & 0.96 & 5 & 0.382103 & 1.59 \\
\hline & GO:0008202 & steroid metabolic & 3 & 0.811342 & 0.99298 & 4 & 0.770535 & 1.00 & 3 & 0.922826 & 0.73 & 5 & 0.599192 & 1.20 \\
\hline & GO:0006694 & steroid biosynthetic & & & & 3 & 0.750237 & 1.13 & & & & 3 & 0.768129 & 1.09 \\
\hline \multirow{3}{*}{$\begin{array}{r}\text { Tissue } \\
\text { Morphogenesis/Gro } \\
\text { wth }\end{array}$} & GO:0060429 & epithelium & 4 & 0.027583 & 5.86335 & 4 & 0.056236 & 4.43 & 4 & 0.059969 & 4.32 & 4 & 0.0614 & 4.28 \\
\hline & GO:0048729 & tissue & 4 & 0.027583 & 5.86335 & 4 & 0.056236 & 4.43 & 4 & 0.059969 & 4.32 & 4 & 0.0614 & 4.28 \\
\hline & GO:0002009 & morphogenesis of & 3 & 0.081574 & 6.15652 & 3 & 0.131471 & 4.65 & 3 & 0.137268 & 4.53 & 3 & 0.139459 & 4.49 \\
\hline \multirow[t]{11}{*}{ Toxin biosynthesis } & GO:1901376 & organic & 5 & 0.004184 & 7.07320 & 6 & 0.001316 & 6.64 & & & & & & \\
\hline & GO:0009403 & toxin biosynthetic & 4 & 0.024683 & 6.09383 & 5 & 0.007544 & 5.96 & 5 & 0.007719 & 5.92 & & & \\
\hline & GO:1901378 & organic & 6 & $8.92 \mathrm{E}-04$ & $\begin{array}{c}7.15234 \\
---\end{array}$ & & & & 6 & $9.19 E-04$ & $\begin{array}{l}7.10 \\
---\end{array}$ & 4 & $5.21 \mathrm{E}-02$ & 4.56 \\
\hline & GO:0009821 & alkaloid & & & & 4 & 0.002391 & 12.3 & 6 & 0.001355 & 6.59 & & & \\
\hline & GO:0035835 & indole alkaloid & & & & 4 & 0.002391 & 12.3 & & & & & & \\
\hline & GO:0035834 & indole alkaloid & & & & 4 & 0.002391 & 12.3 & & & & & & \\
\hline & GO:0045460 & sterigmatocystin & & & & & & & & & & 3 & 0.096438 & 5.55 \\
\hline & GO:0045461 & sterigmatocystin & & & & & & & & & & 3 & 0.096438 & $\begin{array}{l}5.55 \\
-. .\end{array}$ \\
\hline & GO:0042572 & retinol metabolic & & & & & & & & & & 3 & 0.118658 & 4.94 \\
\hline & GO:0034308 & primary alcohol & & & & & & & & & & 4 & 0.131509 & 3.12 \\
\hline & GO:0001523 & retinoid metabolic & & & & & & & & & & 3 & 0.16616 & 4.04 \\
\hline \multicolumn{15}{|c|}{ Downregulated } \\
\hline \multirow[t]{5}{*}{ tRNA Metabolism } & GO:0008033 & tRNA processing & 3 & 0.480084 & 1.84279 & 4 & 0.267003 & 2.19 & 3 & 0.589438 & 1.50 & & & \\
\hline & GO:0006399 & tRNA metabolic & 3 & 0.786562 & 1.03599 & 4 & 0.62622 & 1.23 & 4 & 0.686081 & 1.13 & & & \\
\hline & GO:0034470 & ncRNA processing & 3 & 0.865971 & 0.86047 & 4 & 0.748304 & 1.02 & 4 & 0.800436 & 0.93 & & & \\
\hline & GO:0034660 & ncRNA metabolic & 3 & 0.951106 & 0.64072 & 4 & 0.898786 & 0.76 & 5 & 0.826629 & 0.87 & & & \\
\hline & GO:0006396 & RNA processing & 3 & 0.97995 & 0.52851 & 5 & 0.883403 & 0.78 & 4 & 0.972614 & 0.57 & & & \\
\hline
\end{tabular}




\begin{tabular}{|c|c|c|c|c|c|c|c|c|c|c|c|}
\hline \multirow{5}{*}{$\begin{array}{r}\text { Carbohydrate } \\
\text { Biosynthesis }\end{array}$} & GO:0005976 & polysaccharide & 5 & 0.084753 & 2.95 & & & & & & \\
\hline & GO:0033692 & cellular & 3 & 0.095945 & 5.64 & & & & & & \\
\hline & GO:0044264 & cellular & 3 & 0.188195 & 3.72 & & & & & & \\
\hline & GO:0000271 & polysaccharide & 3 & 0.217308 & 3.38 & & & & & & \\
\hline & GO:0034637 & cellular & 3 & 0.282377 & 2.82 & & & & & & \\
\hline \multirow{5}{*}{$\begin{array}{r}\text { Coenzyme } \\
\text { Biosynthesis }\end{array}$} & GO:0009110 & vitamin & 3 & 0.381694 & 2.24 & & & & & & \\
\hline & GO:0009108 & coenzyme & 3 & 0.453795 & 1.94 & & & & & & \\
\hline & GO:0006766 & vitamin metabolic & 3 & 0.453795 & 1.94 & & & & & & \\
\hline & GO:0051188 & cofactor & 3 & 0.669634 & 1.30 & & & & & & \\
\hline & GO:0006732 & coenzyme & 3 & 0.673396 & 1.29 & & & & & & \\
\hline \multirow{4}{*}{$\begin{array}{r}\text { DNA } \\
\text { Repair/Response to } \\
\text { Abiotic Factor }\end{array}$} & GO:0033554 & cellular response to & 8 & 0.514063 & 1.17 & 6 & 0.870784 & 0.80 & & & \\
\hline & GO:0006281 & DNA repair & 4 & 0.61243 & 1.26 & 3 & 0.863707 & 0.86 & & & \\
\hline & GO:0006974 & response to DNA & 4 & 0.681112 & 1.13 & 4 & 0.738407 & 1.04 & & & \\
\hline & GO:0006259 & DNA metabolic & 4 & 0.884971 & 0.79 & 4 & 0.918017 & 0.72 & & & \\
\hline \multirow{3}{*}{$\begin{array}{r}\text { Carbohydrate } \\
\text { Biosynthesis }\end{array}$} & GO:0006006 & glucose metabolic & & & & & & & 3 & 0.165011 & 4.03 \\
\hline & GO:0019318 & hexose metabolic & & & & & & & 3 & 0.272337 & 2.88 \\
\hline & GO:0005996 & monosaccharide & & & & & & & 3 & 0.359669 & 2.34 \\
\hline
\end{tabular}

\begin{tabular}{|c|c|c|c|c|c|c|c|c|c|c|c|c|c|c|}
\hline & & & \multicolumn{3}{|c|}{ EP155_wt } & \multicolumn{3}{|c|}{ EP155p29 } & \multicolumn{3}{|c|}{ EP155p29stop } & \multicolumn{3}{|c|}{ EP155 $\Delta d c \mid 2$} \\
\hline $\begin{array}{l}\text { Upregulated gene } \\
\text { groups }\end{array}$ & $\begin{array}{c}\text { INTERPR } \\
\text { O/Pfam } \\
\text { id }\end{array}$ & $\begin{array}{l}\text { INTERPRO/Pfam } \\
\text { description }\end{array}$ & $\begin{array}{c}\text { Co } \\
\text { un } \\
t\end{array}$ & Pvalue & $\begin{array}{l}\text { Fold.E } \\
\text { nrichm } \\
\text { ent }\end{array}$ & $\begin{array}{c}\text { Co } \\
\text { un } \\
t\end{array}$ & Pvalue & $\begin{array}{l}\text { Fold.Enr } \\
\text { ichment }\end{array}$ & $\begin{array}{c}\text { Co } \\
\text { un } \\
t\end{array}$ & Pvalue & $\begin{array}{l}\text { Fold } \\
\text {.Enri } \\
\text { chm } \\
\text { ent }\end{array}$ & $\begin{array}{c}\text { Co } \\
\text { un } \\
t\end{array}$ & Pvalue & $\begin{array}{c}\text { Fold } \\
\text {.Enr } \\
\text { ich } \\
\text { men } \\
t\end{array}$ \\
\hline \multirow[t]{5}{*}{ Cytochrome P450 } & IPR01797 & Cytochrome P450, & 14 & 1.7719 & 7.3461 & 18 & $1.05 \mathrm{E}-10$ & 6.89229 & 16 & 8.36121E-09 & 6.24 & 15 & $1.83 \mathrm{E}-07$ & 5.44 \\
\hline & IPR00112 & Cytochrome P450 & 14 & 1.5347 & 6.2520 & 19 & $2.35 \mathrm{E}-10$ & 6.19166 & 17 & 1.34127E-08 & 5.64 & 17 & $3.75 \mathrm{E}-08$ & 5.25 \\
\hline & PF00067 & $\mathrm{p} 450$ & 14 & 1.6743 & 6.2043 & 19 & $2.47 \mathrm{E}-10$ & 6.17177 & 17 & $1.2894 \mathrm{E}-08$ & 5.66 & 17 & $3.93 \mathrm{E}-08$ & 5.23 \\
\hline & IPR01797 & Cytochrome P450, & 13 & 2.3572 & 6.6550 & 18 & 1.7E-10 & 6.72419 & 15 & $1.06105 \mathrm{E}-07$ & 5.71 & 16 & $3.3 \mathrm{E}-08$ & 5.67 \\
\hline & IPR00240 & Cytochrome P450, & 9 & 9.0244 & 5.9031 & 13 & $3.34 \mathrm{E}-07$ & 6.22221 & 12 & $2.36903 \mathrm{E}-06$ & 5.85 & 12 & $4.8 \mathrm{E}-06$ & 5.44 \\
\hline
\end{tabular}




\begin{tabular}{|c|c|c|c|c|c|c|c|c|c|c|c|c|c|c|}
\hline \multirow{5}{*}{$\begin{array}{r}N A D(P)- \\
\text { binding/dehydrogen } \\
\text { ase }\end{array}$} & IPR01604 & $N A D(P)$-binding & 32 & 1.1225 & 3.1831 & 37 & $5.62 \mathrm{E}-08$ & 2.68578 & 30 & $6.09921 \mathrm{E}-05$ & 2.21 & 41 & $1.9 \mathrm{E}-09$ & 2.82 \\
\hline & PF00106 & short chain & 12 & 7.4682 & 4.3094 & 13 & 0.000289 & 3.42192 & 13 & 0.00022917 & 3.50 & 17 & $1.03 \mathrm{E}-06$ & 4.24 \\
\hline & IPR00219 & Short-chain & 13 & 0.0006 & 3.1727 & 18 & $2.93 \mathrm{E}-05$ & 3.20571 & 14 & 0.002912742 & 2.54 & 20 & $3.83 \mathrm{E}-06$ & 3.37 \\
\hline & PF00106 & adh_short & 13 & 0.0007 & 3.1485 & 18 & 3.05E-05 & 3.19542 & 14 & 0.002845875 & 2.54 & 20 & 4.03E-06 & 3.36 \\
\hline & IPR00234 & Glucose/ribitol & 11 & 0.0032 & 2.9984 & 16 & 0.000103 & 3.18259 & 13 & 0.003225864 & 2.63 & 19 & $2.93 \mathrm{E}-06$ & 3.58 \\
\hline \multirow{6}{*}{$\begin{array}{r}\text { Alcohol } \\
\text { dehydrogenase }\end{array}$} & IPR01314 & Alcohol & 8 & 0.0007 & 5.0882 & 8 & 0.004572 & 3.71302 & 6 & 0.056120342 & 2.83 & 11 & $4.58 \mathrm{E}-05$ & 4.84 \\
\hline & IPR00208 & Alcohol & 8 & 0.0007 & 5.0882 & 8 & 0.004572 & 3.71302 & 6 & 0.056120342 & 2.83 & 11 & $4.58 \mathrm{E}-05$ & 4.84 \\
\hline & PF00107 & ADH_zinc_N & 8 & 0.0007 & 5.0494 & 8 & 0.004652 & 3.70109 & 6 & 0.055610557 & 2.84 & 11 & 4.72E-05 & 4.82 \\
\hline & IPR01315 & Alcohol & 6 & 0.0092 & 4.4976 & 7 & 0.008093 & 3.82905 & 5 & 0.099876243 & 2.78 & 9 & 0.00042 & 4.67 \\
\hline & PF08240 & ADH_N & 6 & 0.0095 & 4.4633 & & & & 5 & 0.099159334 & 2.79 & 9 & 0.000431 & 4.65 \\
\hline & IPR00236 & Quinone & & & & 4 & 0.007869 & 8.75212 & & & & & & \\
\hline \multirow{3}{*}{$\begin{array}{r}\text { MFS transporter } \\
\text { superfamily }\end{array}$} & PF07690 & MFS_1 & 10 & 0.0115 & 2.6703 & 18 & 7.79E-06 & 3.52316 & 16 & $9.3673 \mathrm{E}-05$ & 3.21 & 24 & $1.01 \mathrm{E}-09$ & 4.45 \\
\hline & IPR01170 & Major facilitator & 10 & 0.0119 & 2.6568 & 18 & $8.98 \mathrm{E}-06$ & 3.48977 & 16 & 0.000112419 & 3.16 & 24 & $1.25 \mathrm{E}-09$ & 4.41 \\
\hline & PF07690 & Major Facilitator & 5 & 0.2681 & 1.8935 & 12 & 0.000699 & 3.33098 & 9 & 0.02199154 & 2.56 & 17 & $4.66 \mathrm{E}-07$ & 4.47 \\
\hline \multirow{5}{*}{$\begin{array}{r}\text { Fatty } \\
\text { acids/antibiotic } \\
\text { synthesis }\end{array}$} & IPR00908 & Acyl carrier protein- & 5 & 0.0131 & 5.2472 & 6 & 0.007818 & 4.59486 & 5 & 0.035110525 & 3.90 & 5 & 0.044056 & 3.63 \\
\hline & IPR00124 & Condensation & 3 & 0.0785 & 6.2966 & & & & & & & & & \\
\hline & PF00668 & Condensation & 3 & 0.0795 & 6.2486 & & & & & & & & & \\
\hline & IPR00908 & Acyl carrier protein- & 5 & 0.0131 & 5.2472 & 6 & 0.007818 & 4.59486 & 5 & 0.035110525 & 3.90 & 5 & 0.044056 & 3.63 \\
\hline & IPR00616 & Phosphopantethein & 4 & 0.0508 & 4.6641 & & & & 5 & 0.024514836 & 4.33 & 5 & 0.03099 & 4.03 \\
\hline \multirow{5}{*}{$\begin{array}{r}\text { GMC } \\
\text { oxidoreductase }\end{array}$} & PF00732 & GMC & 3 & 0.0527 & 7.8108 & 3 & 0.091707 & 5.72513 & 3 & 0.087817083 & 5.86 & & & \\
\hline & PF05199 & GMC & 3 & 0.0527 & 7.8108 & 3 & 0.091707 & 5.72513 & 3 & 0.087817083 & 5.86 & & & \\
\hline & IPR01213 & Glucose-methanol- & 3 & 0.1241 & 4.8435 & 3 & 0.205838 & 3.53451 & 3 & 0.19986284 & 3.60 & & & \\
\hline & IPR00786 & Glucose-methanol- & 3 & 0.1572 & 4.1977 & 3 & 0.255193 & 3.06324 & 3 & 0.248151898 & 3.12 & & & \\
\hline & PF05199 & GMC_oxred_C & 3 & 0.1591 & 4.1657 & 3 & 0.256381 & 3.05340 & 3 & 0.247224816 & 3.13 & & & \\
\hline \multirow[t]{3}{*}{ 2-Hacid_dh_C } & PF02826 & D-isomer specific 2- & 3 & 0.0943 & 5.6806 & 3 & 0.158579 & 4.16373 & 3 & 0.152320144 & 4.26 & & & \\
\hline & IPR00614 & D-isomer specific 2- & 3 & 0.1743 & 3.9354 & 3 & 0.280002 & 2.87179 & 3 & 0.27247392 & 2.92 & & & \\
\hline & PF02826 & 2-Hacid_dh_C & 3 & 0.1764 & 3.9054 & 3 & 0.281272 & 2.86256 & 3 & 0.271482284 & 2.93 & & & \\
\hline \multirow[t]{3}{*}{$A B C$ transporter } & PF00664 & $A B C$ transporter & & & & & & & & & & 3 & 0.100551 & 5.42 \\
\hline & IPR01787 & $\mathrm{ABC}$ transporter, & 7 & 0.0555 & 2.5331 & 8 & 0.080723 & 2.11258 & 8 & 0.074497047 & 2.15 & 8 & 0.100159 & 2.00 \\
\hline & IPR00343 & $\begin{array}{l}\text { ABC transporter- } \\
\ldots\end{array}$ & 6 & 0.1251 & 2.2488 & 7 & 0.154806 & 1.91452 & 8 & 0.063942637 & 2.23 & 8 & 0.086625 & 2.07 \\
\hline
\end{tabular}




\begin{tabular}{|c|c|c|c|c|c|c|c|c|c|c|c|c|c|c|}
\hline & PF00005 & $\mathrm{ABC}$ tran & 6 & 0.1280 & 2.2316 & 7 & 0.156442 & 1.90837 & 8 & 0.063230363 & 2.23 & 8 & 0.087912 & 2.06 \\
\hline & IPR00114 & $\mathrm{ABC}$ transporter, & 3 & 0.2271 & 3.3140 & 3 & 0.35367 & 2.41834 & 4 & 0.117308608 & 3.28 & 4 & 0.13762 & 3.05 \\
\hline & PF00664 & ABC_membrane & 3 & 0.2297 & 3.2887 & 3 & 0.35515 & 2.41058 & 4 & 0.116634151 & 3.29 & & & \\
\hline \multirow{5}{*}{$\begin{array}{r}\text { Amino acid } \\
\text { permease }\end{array}$} & PF00324 & Amino acid & 3 & 0.1940 & 3.6757 & 3 & 0.306093 & 2.69418 & 3 & 0.295712832 & 2.76 & 6 & 0.004682 & 5.10 \\
\hline & IPR00484 & Amino acid & 3 & 0.2450 & 3.1483 & 3 & 0.377691 & 2.29743 & 3 & 0.368558959 & 2.34 & 6 & 0.009739 & 4.35 \\
\hline & IPR00229 & Amino & 3 & 0.2987 & 2.7376 & 3 & 0.44728 & 1.99776 & 3 & 0.437323258 & 2.03 & 7 & 0.003727 & 4.42 \\
\hline & IPR00484 & Amino acid & 3 & 0.3165 & 2.6236 & 3 & 0.469503 & 1.91452 & 3 & 0.459341841 & 1.95 & 7 & 0.004687 & 4.23 \\
\hline & PF00324 & AA_permease & 3 & 0.3198 & 2.6036 & 3 & 0.471211 & 1.90837 & 3 & 0.457999604 & 1.95 & 7 & 0.00477 & 4.22 \\
\hline \multirow[t]{5}{*}{ Sugar transporter } & IPR00582 & Sugar transporter, & 4 & 0.2300 & 2.3987 & 8 & 0.006432 & 3.50084 & 6 & 0.069280581 & 2.67 & 7 & 0.029904 & 2.90 \\
\hline & IPR00366 & Sugar/inositol & 3 & 0.3518 & 2.4217 & 6 & 0.024069 & 3.53451 & 5 & 0.080443817 & 3.00 & 6 & 0.029468 & 3.35 \\
\hline & IPR00582 & General substrate & 3 & 0.4035 & 2.1712 & 6 & 0.037069 & 3.16887 & 5 & 0.110333484 & 2.69 & 6 & 0.044999 & 3.00 \\
\hline & PF00083 & Sugar_tr & 3 & 0.4072 & 2.1547 & 6 & 0.037506 & 3.15869 & 5 & 0.109554976 & 2.69 & 6 & 0.045589 & 2.99 \\
\hline & PF00083 & Sugar & & & & 4 & 0.154135 & 2.90800 & 3 & 0.390644749 & 2.23 & 4 & 0.172523 & 2.75 \\
\hline \multirow{4}{*}{$\begin{array}{r}\text { Zinc finger, } \mathrm{C} 2 \mathrm{H} 2- \\
\text { like }\end{array}$} & IPR01308 & Zinc finger, $\mathrm{C} 2 \mathrm{H} 2-$ & 5 & 0.1633 & 2.3320 & 7 & 0.069527 & 2.38252 & & & & & & \\
\hline & IPR01588 & Zinc finger, $\mathrm{C} 2 \mathrm{H} 2-$ & 5 & 0.4375 & 1.4780 & 7 & 0.315425 & 1.51004 & & & & & & \\
\hline & IPR00708 & Zinc finger, $\mathrm{C} 2 \mathrm{H} 2-$ & 5 & 0.4588 & 1.4375 & 7 & 0.338934 & 1.46867 & & & & & & \\
\hline & PF00096 & $\mathrm{zf}-\mathrm{C} 2 \mathrm{H} 2$ & 5 & 0.4648 & 1.4266 & 7 & 0.341684 & 1.46396 & & & & & & \\
\hline \multirow[t]{3}{*}{ NmrA } & IPR00803 & NmrA-like & & & & 5 & 0.005757 & 6.38175 & & & & 5 & 0.006951 & 6.05 \\
\hline & PF05368 & NmrA & & & & 5 & 0.005822 & 6.36126 & & & & 5 & 0.00704 & 6.03 \\
\hline & PF05368 & NmrA-like family & & & & 3 & 0.112974 & 5.08900 & & & & 3 & 0.123583 & 4.82 \\
\hline \multirow[t]{3}{*}{ TIM barrel fold } & IPR00115 & NADH flavin & & & & 4 & 0.004723 & 10.2108 & & & & 4 & 0.00549 & 9.68 \\
\hline & PF00724 & Oxidored_FMN & & & & 4 & 0.004766 & 10.1780 & & & & 4 & 0.005547 & 9.65 \\
\hline & IPR01378 & Aldolase-type TIM & & & & 8 & 0.204877 & 1.65580 & & & & 7 & 0.398444 & 1.37 \\
\hline \multirow{4}{*}{$\begin{array}{r}\text { Fatty } \\
\text { acids/antibiotic } \\
\text { synthesis }\end{array}$} & PF00698 & Acyl transferase & & & & 4 & 0.002504 & 12.2136 & 3 & 0.035597164 & 9.39 & & & \\
\hline & PF00109 & Beta-ketoacyl & & & & 4 & 0.004766 & 10.1780 & 3 & 0.051176317 & 7.82 & & & \\
\hline & PF02801 & Beta-ketoacyl & & & & 4 & 0.004766 & 10.1780 & 3 & 0.051176317 & 7.82 & & & \\
\hline & IPR00122 & Acyl transferase & & & & 4 & 0.011988 & 7.65810 & & & & & & \\
\hline \multirow[t]{3}{*}{ FAD binding domain } & PF01565 & FAD binding domain & & & & 3 & 0.112974 & 5.08900 & 3 & 0.108294406 & 5.21 & 4 & 0.019937 & 6.43 \\
\hline & IPR00609 & FAD linked oxidase, & & & & 3 & 0.157766 & 4.17714 & 3 & 0.152952944 & 4.25 & 4 & 0.035009 & 5.28 \\
\hline & PF01565 & FAD_binding_4 & & & & 3 & 0.158579 & 4.16373 & 3 & 0.152320144 & 4.26 & 4 & 0.035336 & 5.26 \\
\hline
\end{tabular}




\begin{tabular}{|c|c|c|c|c|c|c|c|c|c|c|c|}
\hline & IPR01616 & FAD-binding, type 2 & 3 & 0.181562 & 3.82905 & 3 & 0.176158878 & 3.90 & 4 & 0.044362 & 4.84 \\
\hline \multirow{7}{*}{$\begin{array}{r}\text { Glutathione S- } \\
\text { transferase }\end{array}$} & PF02798 & Glutathione S- & 3 & 0.23153 & 3.27150 & 3 & 0.223048624 & 3.35 & & & \\
\hline & PF00043 & Glutathione S- & 3 & 0.23153 & 3.27150 & 3 & 0.223048624 & 3.35 & & & \\
\hline & IPR00404 & Glutathione S- & 3 & 0.280002 & 2.87179 & 3 & 0.27247392 & 2.92 & 4 & 0.092307 & 3.63 \\
\hline & PF00043 & GST_C & 3 & 0.281272 & 2.86256 & 3 & 0.271482284 & 2.93 & 4 & 0.093086 & 3.61 \\
\hline & IPR00404 & Glutathione S- & 3 & 0.304747 & 2.70286 & & & & 4 & 0.106634 & 3.41 \\
\hline & PF02798 & GST_N & & & & 3 & 0.295712832 & 2.76 & 4 & 0.107516 & 3.40 \\
\hline & IPR01793 & Glutathione S- & & & & & & & 4 & 0.13762 & 3.05 \\
\hline \multirow{4}{*}{$\begin{array}{r}\text { Haem peroxidase, } \\
\text { plant/fungal/bacteri } \\
\text { al }\end{array}$} & PF00141 & peroxidase & & & & 3 & 0.087817083 & 5.86 & 3 & 0.100551 & 5.42 \\
\hline & IPR01979 & Peroxidases heam- & & & & 3 & 0.088209941 & 5.85 & 3 & 0.099927 & 5.44 \\
\hline & IPR00201 & Haem peroxidase, & & & & 3 & 0.088209941 & 5.85 & 3 & 0.099927 & 5.44 \\
\hline & IPR01979 & Peroxidase, active & & & & 3 & 0.108767189 & 5.20 & 3 & 0.122837 & 4.84 \\
\hline \multirow{3}{*}{$\begin{array}{r}\text { Protein tyrosine } \\
\text { kinase }\end{array}$} & PF07714 & Protein tyrosine & & & & 3 & 0.068685537 & 6.70 & & & \\
\hline & PF07714 & Pkinase_Tyr & & & & 3 & 0.152320144 & 4.26 & & & \\
\hline & IPR00124 & Tyrosine protein & & & & 3 & 0.152952944 & 4.25 & & & \\
\hline \multirow[t]{5}{*}{ DNA/RNA helicase } & PF00176 & SNF2_N & & & & 4 & 0.161648322 & 2.84 & 4 & 0.190224 & 2.63 \\
\hline & IPR00033 & SNF2-related & & & & 4 & 0.162527448 & 2.83 & 4 & 0.188822 & 2.64 \\
\hline & IPR01402 & Helicase, & & & & 7 & 0.357397627 & 1.43 & 4 & 0.902256 & 0.76 \\
\hline & PF00271 & Helicase_C & & & & 7 & 0.366755614 & 1.42 & & & \\
\hline & IPR00165 & DNA/RNA helicase, & & & & 7 & 0.369042097 & 1.41 & & & \\
\hline \multirow{3}{*}{$\begin{array}{l}\text { Ammonium } \\
\text { transporte }\end{array}$} & IPR01804 & Ammonium & & & & & & & 3 & 0.01346 & 14.5 \\
\hline & IPR00190 & Ammonium & & & & & & & 3 & 0.01346 & 14.5 \\
\hline & PF00909 & Ammonium_transp & & & & & & & 3 & 0.013555 & 14.4 \\
\hline \multirow[t]{5}{*}{ Carboxylesterase } & PF00135 & Carboxylesterase & & & & & & & 4 & 0.044768 & 4.82 \\
\hline & IPR01982 & Carboxylesterase & & & & & & & 4 & 0.054819 & 4.47 \\
\hline & IPR00201 & Carboxylesterase, & & & & & & & 4 & 0.066334 & 4.15 \\
\hline & IPR01981 & Carboxylesterase & & & & & & & 4 & 0.066334 & 4.15 \\
\hline & PF00135 & COesterase & & & & & & & 4 & 0.066917 & 4.13 \\
\hline \multirow{2}{*}{$\begin{array}{r}\text { OPT oligopeptide } \\
\text { transporter protein }\end{array}$} & IPR00464 & Tetrapeptide & & & & & & & 3 & 0.040906 & 8.71 \\
\hline & PF03169 & OPT oligopeptide & & & & & & & 3 & 0.058998 & 7.23 \\
\hline
\end{tabular}




\begin{tabular}{|c|c|c|c|c|c|c|c|c|c|c|c|c|c|c|}
\hline & IPR00481 & Oligopeptide & & & & & & & & & & 3 & 0.07841 & 6.22 \\
\hline & PF03169 & OPT & & & & & & & & & & 3 & 0.078912 & 6.20 \\
\hline \multirow{3}{*}{$\begin{array}{r}\text { Fatty } \\
\text { acids/antibiotic } \\
\text { synthesis }\end{array}$} & IPR00616 & Phosphopantethein & & & & & & & & & & 4 & 0.13762 & 3.05 \\
\hline & PF00550 & PP-binding & & & & & & & & & & 4 & 0.138711 & 3.04 \\
\hline & IPR01007 & Amino acid & & & & & & & & & & 3 & 0.146847 & 4.35 \\
\hline \multirow[t]{3}{*}{ ATP-grasp fold } & IPR01381 & Pre-ATP-grasp fold & & & & & & & & & & 3 & 0.171699 & 3.96 \\
\hline & IPR01176 & ATP-grasp fold & & & & & & & & & & 3 & 0.197167 & 3.63 \\
\hline & IPR01381 & ATP-grasp fold, & & & & & & & & & & 3 & 0.27537 & 2.90 \\
\hline \multirow{3}{*}{$\begin{array}{l}\text { FAD dependent } \\
\text { oxidoreductase }\end{array}$} & PF01266 & FAD dependent & & & & & & & & & & 3 & 0.250492 & 3.10 \\
\hline & IPR00607 & FAD dependent & & & & & & & & & & 3 & 0.40353 & 2.17 \\
\hline & PF01266 & DAO & & & & & & & & & & 3 & 0.405337 & 2.17 \\
\hline \multirow[t]{3}{*}{ SET domain } & PF00856 & SET domain & & & & & & & & & & 3 & 0.380336 & 2.28 \\
\hline & IPR00121 & SET & & & & & & & & & & 3 & 0.451899 & 1.98 \\
\hline & PF00856 & SET & & & & & & & & & & 3 & 0.453806 & 1.97 \\
\hline \multirow[t]{2}{*}{ DNA/RNA helicase } & PF00176 & SNF2 family N- & & & & & & & & & & 3 & 0.172689 & 3.94 \\
\hline & PF00271 & Helicase conserved & & & & & & & & & & 4 & 0.717734 & 1.09 \\
\hline \multicolumn{15}{|c|}{ Downregulated } \\
\hline \multirow[t]{5}{*}{ Sugar transporter } & IPR00366 & Sugar/inositol & 7 & 1.8493 & 17.537 & 5 & 0.000771 & 11.5936 & 6 & $6.88398 \mathrm{E}-05$ & 13.2 & & & \\
\hline & IPR00582 & General substrate & 7 & 3.6789 & 15.722 & 5 & 0.001179 & 10.3943 & 6 & 0.000119274 & 11.8 & & & \\
\hline & IPR00582 & Sugar transporter, & 9 & 3.4102 & 16.749 & 7 & $1.84 \mathrm{E}-05$ & 12.0574 & 8 & 1.68687E-06 & 13.0 & & & \\
\hline & PF00083 & Sugar_tr & 7 & 4.2017 & 15.368 & 5 & 0.001331 & 10.0628 & 6 & 0.000145119 & 11.3 & & & \\
\hline & PF00083 & Sugar & 4 & 0.0039 & 12.127 & 4 & 0.005033 & 11.1170 & 5 & 0.000470284 & 13.0 & & & \\
\hline \multirow[t]{5}{*}{ Cytochrome P450 } & IPR00112 & Cytochrome P450 & 5 & 0.0053 & 6.9295 & 4 & 0.041284 & 5.13082 & 5 & 0.008492515 & 6.08 & 4 & 0.022182 & 6.51 \\
\hline & IPR00240 & Cytochrome P450, & 4 & 0.0121 & 8.1422 & 3 & 0.096252 & 5.65192 & 5 & 0.002085706 & 8.94 & 3 & 0.063342 & 7.17 \\
\hline & IPR01797 & Cytochrome P450, & 3 & 0.1227 & 4.8853 & 3 & 0.139389 & 4.52154 & 4 & 0.031225909 & 5.72 & 4 & 0.014407 & 7.65 \\
\hline & IPR01797 & Cytochrome P450, & 4 & 0.0237 & 6.3549 & 3 & 0.145045 & 4.41126 & 5 & 0.00521691 & 6.98 & 4 & 0.015403 & 7.47 \\
\hline & PF00067 & p450 & 5 & 0.0058 & 6.7734 & 4 & 0.044776 & 4.96719 & 5 & 0.009799811 & 5.84 & 4 & 0.022846 & 6.44 \\
\hline \multirow{3}{*}{$\begin{array}{r}\text { MFS transporter } \\
\text { superfamily }\end{array}$} & IPR01170 & Major facilitator & 4 & 0.1176 & 3.2981 & 6 & 0.009264 & 4.57877 & 5 & 0.047202136 & 3.62 & 3 & 0.270776 & 2.90 \\
\hline & PF07690 & MFS_1 & 4 & 0.1203 & 3.2651 & 6 & 0.010043 & 4.48958 & 5 & 0.051496978 & 3.52 & 3 & 0.270252 & 2.91 \\
\hline & PF07690 & Major Facilitator & 3 & 0.2100 & 3.4729 & 4 & 0.065841 & 4.24469 & 3 & 0.260998835 & 2.99 & 3 & 0.16041 & 4.12 \\
\hline
\end{tabular}




\begin{tabular}{|c|c|c|c|c|c|c|c|c|}
\hline \multirow[t]{4}{*}{ Glycoside hydrolase } & IPR00074 & Glycoside & 4 & 0.000846 & 20.0957 & & & \\
\hline & IPR00662 & Parallel beta-helix & 4 & 0.000846 & 20.0957 & & & \\
\hline & IPR01233 & Pectin lyase fold & 4 & 0.003984 & 12.0574 & & & \\
\hline & PF00295 & Glyco_hydro_28 & 4 & 0.00093 & 19.4548 & & & \\
\hline \multirow{5}{*}{$\begin{array}{r}\text { Alcohol } \\
\text { dehydrogenase }\end{array}$} & IPR00208 & Alcohol & & & & 5 & 0.002342262 & 8.67 \\
\hline & IPR00232 & Alcohol & & & & 5 & 0.000213651 & 15.9 \\
\hline & IPR01315 & Alcohol & & & & 5 & 0.001252041 & 10.2 \\
\hline & PF08240 & ADH_N & & & & 5 & 0.001460437 & 9.80 \\
\hline & PF08240 & Alcohol & & & & 4 & 0.001791232 & 15.6 \\
\hline
\end{tabular}


Table S 3. Orthologs differentially expressed during HI in $P$. anserina and N. crassa, and barrage in C. parasitica.

\begin{tabular}{|c|c|c|c|c|c|c|c|c|c|c|c|c|c|}
\hline Nc_Id & Pa_Id & CP_Id & $\begin{array}{l}\text { UP_prot } \\
\text { einld }\end{array}$ & $\begin{array}{l}\text { TrEMBL_ } \\
\text { Id }\end{array}$ & Clusters & NC & PA & $\begin{array}{l}\text { EP15 } \\
5 w t \\
\text { mix }\end{array}$ & $\begin{array}{l}\text { EP15 } \\
\text { 5_p2 } \\
\text { 9mix }\end{array}$ & $\begin{array}{l}\text { EP15 } \\
\text { 5_p2 } \\
\text { 9sto } \\
\text { pmix }\end{array}$ & $\begin{array}{l}\text { EP15 } \\
5 \text { _dcl } \\
2 \mathrm{mix}\end{array}$ & $\begin{array}{l}\text { Mea } \\
\mathrm{n}\end{array}$ & Protein.names UniProt \\
\hline NCU04736 & Pa_4_856 & 336241 & Q9HDW7 & A0A084 & Cluster1 & 5.05 & 2.22 & 1.22 & 1.53 & 2.62 & 1.36 & 1.68 & Calcium-transporting ATPase 2 (EC 3.6.3.8) \\
\hline NCU05555 & Pa_4_360 & 342654 & Q9GLY5 & L2GFX9 & Cluster1 & 1.95 & 4.72 & 2.27 & 3.65 & 3.58 & 2.87 & 3.09 & Inter-alpha-trypsin inhibitor heavy chain \\
\hline NCU01866 & Pa_1_221 & 15980 & Q01578 & K2RHJ5 & Cluster1 & 1.82 & 1.00 & 1.40 & 2.37 & 3.42 & 1.57 & 2.19 & Gluconolactonase (EC 3.1.1.17) (D-glucono- \\
\hline NCU09798 & Pa_1_230 & 340915 & V5NDL4 & C7ZIC7 & Cluster1 & 1.58 & 2.00 & 3.14 & 1.93 & 3.02 & 3.04 & 2.78 & Pyranose dehydrogenase (PDH) (EC \\
\hline NCU09798 & Pa_5_487 & 335584 & Q7X2H8 & R8BHUO & Cluster1 & 1.58 & -1.43 & 2.30 & 2.27 & 2.31 & 2.36 & 2.31 & Choline oxidase (EC 1.1.3.17) \\
\hline NCU07080 & Pa_4_467 & 257749 & P04387 & A0A084 & Cluster1 & 1.31 & 1.57 & 0.77 & 1.13 & 0.50 & 2.31 & 1.18 & Galactose/lactose metabolism regulatory \\
\hline NCU00695 & Pa_1_188 & 354805 & Q941V3 & R8BJQ0 & Cluster1 & 1.28 & 3.54 & 1.54 & 2.32 & 2.00 & 1.30 & 1.79 & Probable metal-nicotianamine transporter \\
\hline NCU04452 & Pa_6_633 & 278980 & Q4WZ70 & K1WTU1 & Cluster1 & 1.16 & 1.07 & 1.50 & 2.41 & 1.09 & 2.95 & 1.99 & Chanoclavine-I aldehyde reductase (EC \\
\hline NCU09040 & Pa_5_117 & 356334 & Q9COY6 & G9NFQ3 & Cluster1 & -1.00 & -1.17 & 2.35 & 1.10 & 2.21 & 1.43 & 1.77 & Zinc-type alcohol dehydrogenase-like \\
\hline NCU03863 & Pa_1_197 & 355831 & C8ZI10 & S7ZJX2 & Cluster1 & -1.07 & 2.38 & 2.15 & 2.31 & 1.89 & 2.64 & 2.24 & Sphingoid long-chain base transporter \\
\hline NCU09821 & Pa_1_125 & 337218 & Q10216 & H6CB95 & Cluster1 & -1.07 & 1.23 & 0.10 & 2.54 & 2.16 & 1.78 & 1.64 & Uncharacterized oxidoreductase $\mathrm{C} 4 \mathrm{H} 3.08$ \\
\hline NCU01419 & Pa_2_850 & 99498 & Q53FA7 & L7HYU8 & Cluster1 & -1.16 & -1.57 & 1.27 & 1.72 & 0.73 & 2.50 & 1.55 & Quinone oxidoreductase PIG3 (EC 1.-.-.-) \\
\hline NCU06861 & Pa_0_146 & 333209 & & V2WQX3 & Cluster1 & -1.20 & -1.05 & 0.85 & 0.58 & 1.80 & 3.15 & 1.60 & \\
\hline NCU08052 & Pa_6_109 & 245539 & C8ZJM1 & X0GVA9 & Cluster1 & -1.27 & 2.09 & 2.40 & 2.83 & 2.69 & 2.11 & 2.51 & Aquaporin-1 \\
\hline NCU05390 & Pa_6_514 & 295517 & P23641 & A0A010R & Cluster1 & -1.42 & -1.33 & 1.23 & 2.37 & 1.82 & 2.03 & 1.86 & Mitochondrial phosphate carrier protein \\
\hline NCU02408 & Pa_5_210 & 264067 & Q6CMK7 & R1EUZ1 & Cluster1 & -1.94 & -1.59 & 2.16 & 2.63 & 2.48 & 1.91 & 2.29 & Delta(8)-fatty-acid desaturase (EC \\
\hline NCU00821 & Pa_1_520 & 347965 & P39932 & M7T407 & Cluster1 & -2.17 & -3.38 & 0.74 & -0.12 & 0.37 & 2.20 & 0.80 & Sugar transporter STL1 \\
\hline NCU00821 & Pa_1_520 & 337702 & P39932 & Q0CMX3 & Cluster1 & -2.17 & -3.38 & 2.31 & 2.47 & 1.90 & 2.75 & 2.36 & Sugar transporter STL1 \\
\hline NCU02801 & Pa_1_799 & 343748 & 094327 & A0A084R & Cluster1 & -2.61 & 1.69 & 3.09 & 2.97 & 2.30 & 2.19 & 2.64 & Translocator protein homolog \\
\hline NCU02478 & Pa_5_910 & 293608 & Q9Y719 & A0A066 & Cluster1 & -2.67 & 3.12 & 2.45 & 2.46 & 1.89 & 2.67 & 2.37 & Cell wall alpha-1,3-glucan synthase mok13 \\
\hline NCU07474 & Pa_1_681 & 263261 & Q4WZ69 & A0A010 & Cluster1 & -2.69 & 2.83 & 2.45 & 3.07 & 2.47 & 2.09 & 2.52 & Festuclavine dehydrogenase (EC 1.5.1.44) \\
\hline NCU07649 & Pa_4_144 & 231232 & & A0A010 & Cluster1 & -2.85 & 1.23 & 1.78 & 1.60 & 2.08 & 1.13 & 1.65 & unknown_gene \\
\hline NCU00260 & Pa_7_456 & 47533 & Q04894 & G2QGG1 & Cluster1 & -2.96 & -1.58 & 3.08 & 3.27 & 1.80 & 3.04 & 2.80 & DP-dependent alcohol dehydrogenase 6 \\
\hline NCU09409 & Pa_4_510 & 106290 & P9WK87 & F9WYK7 & Cluster1 & -3.14 & 1.82 & 1.88 & 1.96 & 1.93 & 2.32 & 2.02 & Carboxylesterase NIhH (EC 3.1.1.1) \\
\hline NCU10021 & Pa_5_480 & 345209 & Q92253 & F9G0U8 & Cluster1 & -3.28 & -1.75 & 1.99 & 2.44 & 0.71 & 0.94 & 1.52 & Probable glucose transporter rco-3 \\
\hline NCU08418 & Pa_3_105 & 71314 & Q70J59 & M7SXF5 & Cluster1 & -3.88 & 1.40 & 2.36 & 2.68 & 4.31 & 2.53 & 2.97 & Tripeptidyl-peptidase sed2 (EC 3.4.14.-) \\
\hline NCU00585 & Pa_1_152 & 337116 & B2АТВ0 & A0A090C & Cluster1 & -3.98 & 1.26 & 1.14 & 1.99 & 2.01 & 2.60 & 1.94 & Bifunctional lycopene cyclase/phytoene \\
\hline NCU09648 & Pa_3_570 & 67132 & Q9URW9 & R1EBH8 & Cluster1 & -4.12 & 1.01 & 3.57 & 3.47 & 3.55 & 3.02 & 3.40 & Putative aldehyde dehydrogenase-like \\
\hline NCU09648 & Pa_3_570 & 75530 & C3MIE5 & R1EBH8 & Cluster1 & -4.12 & 1.01 & 3.01 & 3.34 & 3.11 & 1.75 & 2.80 & D/DP-dependent betaine aldehyde \\
\hline NCU05780 & Pa_1_510 & 357090 & Q9Y7Q2 & G2Q0J5 & Cluster15 & -1.04 & 4.09 & 6.52 & 6.76 & 3.49 & 9.86 & 6.66 & Glutathione S-transferase 1 (EC 2.5.1.18) \\
\hline NCU07055 & Pa_4_456 & 327822 & P86491 & SOEP39 & Cluster2 & 1.72 & -2.50 & -1.56 & -0.81 & -1.22 & -2.66 & -1.56 & 6-hydroxynicotinate 3-monooxygenase (EC \\
\hline NCU09798 & Pa_1_230 & 237359 & Q7MF12 & SOE8E9 & Cluster2 & 1.58 & 2.00 & -2.70 & -2.19 & -1.57 & -2.33 & -2.20 & Oxygen-dependent choline dehydrogenase \\
\hline
\end{tabular}




\begin{tabular}{|c|c|c|c|c|c|c|c|c|c|c|c|c|c|}
\hline NCU01241 & Pa_2_329 & 247929 & Q9VA73 & R8BUG6 & Cluster2 & 1.22 & -2.63 & -1.49 & -2.21 & -2.27 & -1.30 & -1.82 & Calcium-binding mitochondrial carrier \\
\hline NCU04122 & Pa_2_121 & 353782 & & М7T0P6 & Cluster2 & -1.47 & -2.03 & -2.17 & -2.20 & -2.01 & -2.19 & -2.14 & \\
\hline NCU07240 & Pa_2_140 & 357339 & 095154 & A0A024S & Cluster2 & -2.36 & -1.04 & -2.84 & -0.53 & 0.13 & -0.47 & -0.93 & Aflatoxin B1 aldehyde reductase member 3 \\
\hline NCU04721 & Pa_4_695 & 100328 & Q304B9 & U7Q0G1 & Cluster2 & -2.60 & -2.88 & -2.16 & -2.06 & -2.46 & -2.19 & -2.22 & Neutral ceramidase (N-CDase) (NCDase) \\
\hline NCU02478 & Pa_5_910 & 36475 & Q09854 & R8BQS1 & Cluster2 & -2.67 & 3.12 & -1.71 & -1.92 & -2.40 & -0.05 & -1.52 & Cell wall alpha-1,3-glucan synthase mok11 \\
\hline NCU05789 & $\mathrm{Pa}+4 \_870$ & 47283 & P45798 & R8BJI3 & Cluster2 & -3.00 & 1.95 & -1.88 & -2.09 & -1.67 & -1.50 & -1.78 & Beta-glucanase (EC 3.2.1.73) (1,3-1,4-beta- \\
\hline NCU09287 & Pa_5_452 & 252856 & Q9XIH6 & W9WLT8 & Cluster2 & -3.09 & -1.36 & -1.64 & -1.65 & -1.72 & -3.43 & -2.11 & Putative polyol transporter 2 \\
\hline NCU09976 & Pa_1_221 & 33426 & Q00017 & TOKGT6 & Cluster2 & -3.16 & -2.49 & 0.04 & -0.11 & 0.09 & -2.07 & -0.51 & Rhamnogalacturonan acetylesterase \\
\hline NCU07454 & Pa_1_979 & 357001 & & M4FX61 & Cluster2 & -3.18 & -2.90 & -2.15 & -1.44 & -2.14 & -2.74 & -2.12 & \\
\hline NCU08418 & Pa_3_105 & 342166 & Q70GH4 & A0A074 & Cluster2 & -3.88 & 1.40 & -2.36 & -2.88 & -2.17 & -2.53 & -2.49 & Tripeptidyl-peptidase sed3 (EC 3.4.14.-) \\
\hline NCU08173 & Pa_5_680 & 106417 & A3LNF8 & L2FGJ5 & Cluster2 & -4.37 & 1.95 & -2.09 & -2.16 & -2.07 & -1.86 & -2.04 & Kynurenine 3-monooxygenase (EC \\
\hline NCU00732 & Pa_1_101 & 327709 & 013317 & W3XIA9 & Cluster2 & -5.30 & -1.98 & -2.63 & -2.93 & -1.30 & -1.25 & -2.03 & Isotrichodermin C-15 hydroxylase (EC \\
\hline NCU03903 & Pa_5_130 & 264062 & 014158 & T5APL5 & Cluster4 & 2.24 & 1.13 & 4.78 & 4.72 & 5.04 & 3.42 & 4.49 & $A B$ hydrolase superfamily protein $C 4 A 8.06 c$ \\
\hline NCU09244 & Pa_1_113 & 76171 & P97819 & Q2GN73 & Cluster4 & 1.97 & 2.22 & 3.27 & 3.44 & 2.99 & 3.85 & 3.39 & 85/88 kDa calcium-independent \\
\hline NCU07546 & Pa_7_777 & 271042 & P36619 & U7PN06 & Cluster4 & 1.34 & 1.62 & 2.50 & 4.50 & 3.54 & 1.11 & 2.91 & Leptomycin B resistance protein pmd1 \\
\hline NCU09103 & Pa_1_235 & 262538 & Q12612 & W3XLNO & Cluster4 & 1.06 & 3.07 & 2.42 & 4.40 & 4.31 & 4.30 & 3.86 & Trichodiene oxygenase (EC 1.14.---) \\
\hline NCU01419 & Pa_2_850 & 333952 & Q53FA7 & C5P864 & Cluster4 & -1.16 & -1.57 & 4.82 & 5.69 & 6.12 & 4.56 & 5.30 & Quinone oxidoreductase PIG3 (EC 1.-.-.-) \\
\hline NCU05045 & Pa_1_638 & 220882 & Q08777 & U1GMV6 & Cluster4 & -1.74 & -1.29 & 3.90 & 3.94 & 3.75 & 2.54 & 3.53 & Riboflavin transporter $\mathrm{MCH} 5$ \\
\hline NCU05045 & Pa_5_705 & 64984 & Q08777 & R1GB39 & Cluster4 & -1.74 & -1.89 & 2.68 & 4.18 & 3.55 & 4.02 & 3.61 & Riboflavin transporter $\mathrm{MCH} 5$ \\
\hline NCU07080 & Pa_4_467 & 276377 & P04387 & A0A024S & Cluster6 & 1.31 & 1.57 & 3.27 & 4.64 & 2.19 & 6.82 & 4.23 & Galactose/lactose metabolism regulatory \\
\hline NCU07080 & Pa_4_467 & 356997 & P04387 & A0A066 & Cluster6 & 1.31 & 1.57 & 2.02 & 3.43 & 1.35 & 5.47 & 3.07 & Galactose/lactose metabolism regulatory \\
\hline NCU07080 & Pa_4_467 & 57319 & & W3XJB8 & Cluster6 & 1.31 & 1.57 & 2.28 & 3.40 & 1.32 & 5.81 & 3.20 & \\
\hline NCU07055 & Pa_4_456 & 320149 & P86491 & Q7S5W2 & Cluster8 & 1.72 & -2.50 & 6.30 & 8.55 & 7.61 & 4.90 & 6.84 & 6-hydroxynicotinate 3-monooxygenase (EC \\
\hline NCU09103 & Pa_3_290 & 251671 & Q12612 & G2R1V6 & Cluster8 & 1.06 & 1.26 & 6.61 & 6.74 & 6.10 & 6.07 & 6.38 & Trichodiene oxygenase (EC 1.14.-.-) \\
\hline NCU05780 & Pa_1_510 & 58765 & Q9Y7Q2 & M7T1S0 & Cluster8 & -1.04 & 4.09 & 7.30 & 7.49 & 6.94 & 5.84 & 6.90 & Glutathione S-transferase 1 (EC 2.5.1.18) \\
\hline NCU02031 & Pa_0_160 & 254007 & Q12587 & J5JX77 & Cluster8 & -2.09 & 3.79 & 6.30 & 6.78 & 6.50 & 5.45 & 6.26 & Cytochrome P450 52C2 (EC 1.14.14.-) \\
\hline NCU08173 & Pa_5_680 & 355284 & B6D1N4 & B2W1A7 & Cluster8 & -4.37 & 1.95 & 5.22 & 4.60 & 4.13 & 5.99 & 4.98 & FAD-dependent urate hydroxylase (EC \\
\hline
\end{tabular}

